\title{
Avian $\beta$-Defensins as Antimicrobial and Immunomodulatory Agents
}

\author{
A Dissertation \\ Presented to \\ the Faculty of the Graduate School \\ at the University of Missouri-Columbia \\ In Partial Fulfillment \\ of the Requirements for the Degree \\ Doctor of Philosophy
}

by

MING YANG

Dr. Shuping Zhang, Dissertation Advisor

May 2018 
The undersigned, appointed by the dean of the Graduate School, have examined the dissertation entitled

\title{
Avian $\beta$-Defensins as Antimicrobial and Immunomodulatory Agents
}

\author{
Presented by Ming Yang,
} a candidate for the degree of Doctor of Philosophy, and hereby certify that, in their opinion, it is worthy of acceptance.

Professor Shuping Zhang

Professor Charles R. Brown

Associate Clinical Professor Thomas J. Reilly

Associate Professor Guoquan Zhang

Associate Clinical Professor Michael Z. Zhang 


\section{DEDICATION}

This dissertation is dedicated to my amazing wife Chunye Zhang, whose love, care, and support throughout the entire research study made this hard work possible, and I love you and would like to spend life with you; to my parents, parents-in-law, my grandparents, sisters, and brothers, your care and encouragement inspired me along the way; to my friends Randy and Brenda, your friendship and help added much joy in my life during this study. 


\section{ACKNOWLEDGMENTS}

Firstly, I want to thank my advisor Dr. Shuping Zhang for her guidance and support. She always encouraged me to think and work as a scientist, and provided me much freedom to design and finish the research project independently.

I would also like to thank Dr. Charles R. Brown, Dr. Thomas J. Reilly, Dr. Guoquan Zhang and Dr. Michael Z. Zhang for serving as my committee members. They are smart scientists, and their advice and feedback made me a much stronger researcher, which is beneficial for my career development.

Additionally, I want to thank Dr. George C. Stewart for his great lessons and encouragement in career development, thank Dr. George E. Rottinghaus for his help in HPLC analysis, thank Dr. Michael Calcutt and Dr. Christopher P. Baines for sharing related research information, thank Dr. Sarah A. Hansen, Dr. Catherine Chambers, and Ms. Sherrie E. Neff for their help at the animal work, thank Dr. Mitchell W. Jefferson for his help in histopathology analysis, thank graduate student Chunye Zhang for her great help in research work and proposal design, and thank Dr. Zhenyu Shen for his assistance in managing the lab.

Furthermore, I am grateful to all of those from Veterinary Medical Diagnostic Lab for their technical support and service, with whom I have had the chance to work with during this project especially for Dr. William H. Fales, Ms. Irene Ganjam, Ms. Geri Hegstad, Mr. Michael Wimmenauer, Ms. Karen Huggins, and Ms. Raina Cornell.

Last but not least, I want to acknowledge the Department of Veterinary Pathobiology of College of Veterinary Medicine for the permission to study in Mizzou and program support, and thank Ms. Marie E. Schlup and Ms. Anne E, Chedwidden for their help. 


\section{Table of Contents}

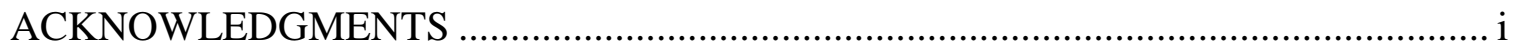

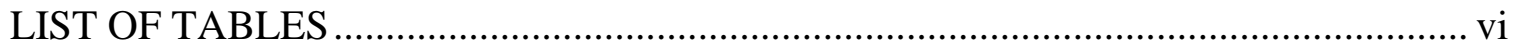

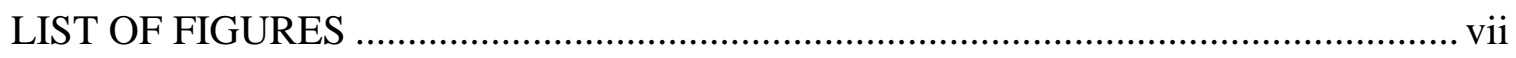

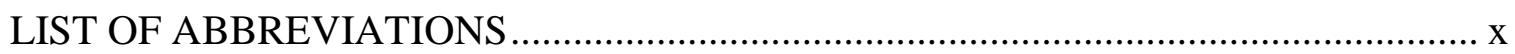

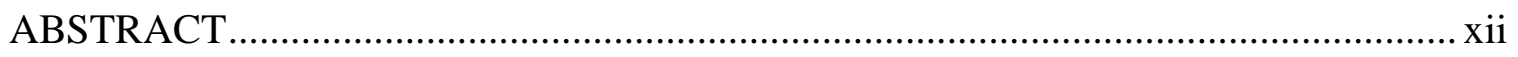

CHAPTER 1: Avian Beta-Defensins: Potent Bactericidal Agents..................................... 1

1. Host Antimicrobial Peptides (AMPs) …………….......................................... 1

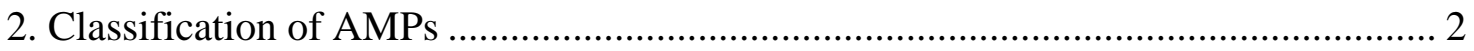

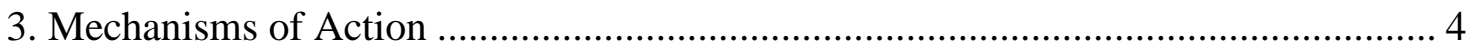

4. Avian $\beta$-defensin (AvBDs) and Their Biological Functions......................................... 7

5. Strategies for Developing Novel AMPs............................................................ 13

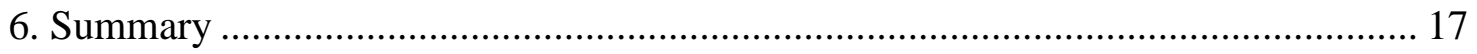

CHAPTER 2: Biological Functions of Avian $\beta$-Defensins (AvBDs): AvBD-6 and AvBD-

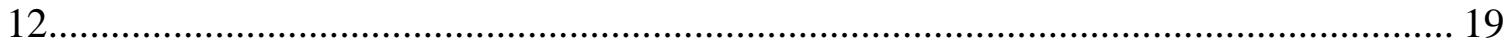

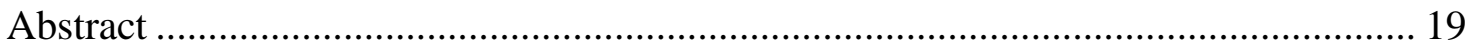

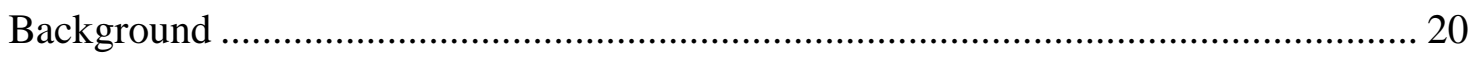

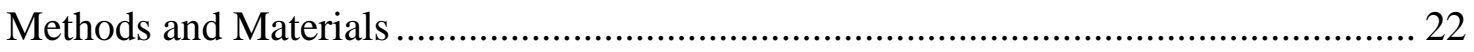

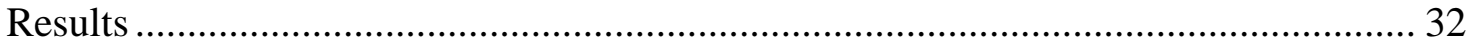

Antimicrobial activity of AvBD-6 and AvBD-12 ……........................................... 32 
The ability of AvBD to neutralize LPS

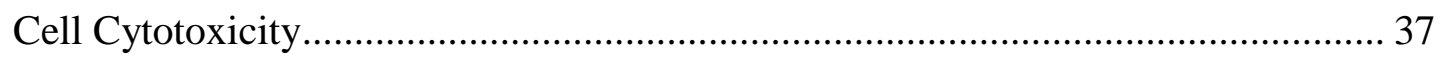

Expression of CCR2-GFP/CCR6-GFP proteins in CHO-K1 cells.......................... 40

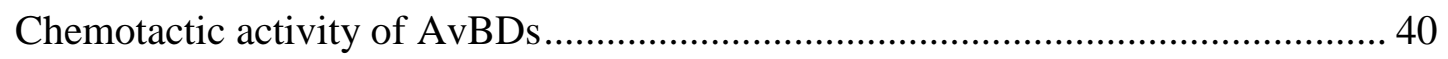

The antimicrobial, chemotactic, and neutralizing LPS activities of reduced AvBDs 43

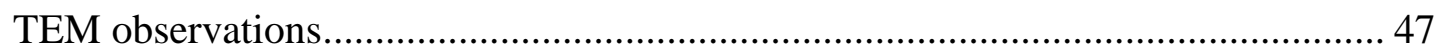

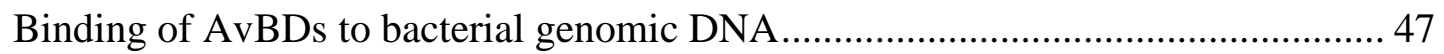

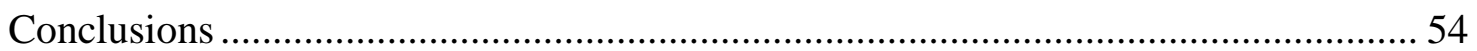

CHAPTER 3: Novel Synthetic Analogs of Avian $\beta$-Defensin-12: the Roles of Charge, Hydrophobicity, and Disulfide Bonds in Biological Functions .................................... 55

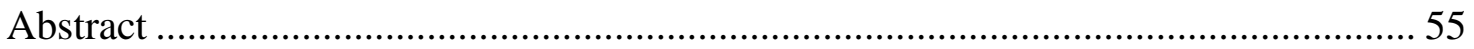

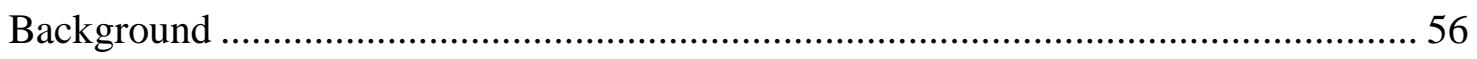

Methods and Materials ..................................................................................... 57

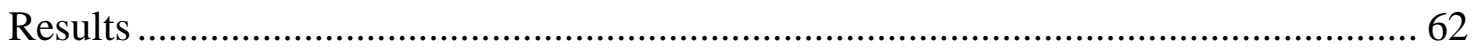

Structural characteristics of AvBD-6 and AvBD-12 and their analogs....................6 62

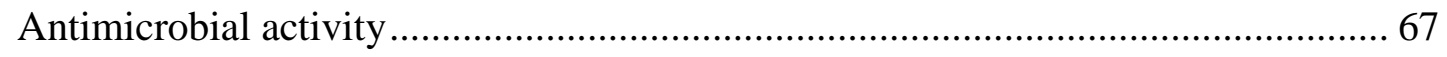

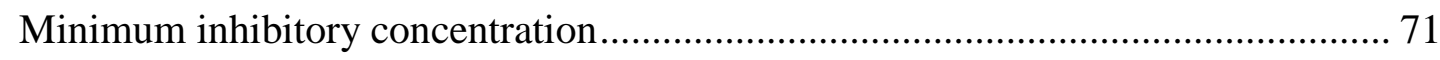

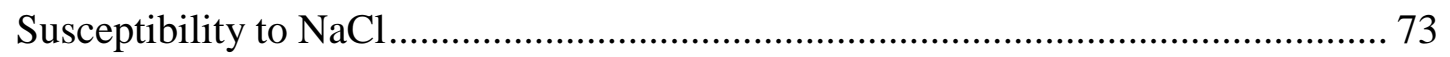

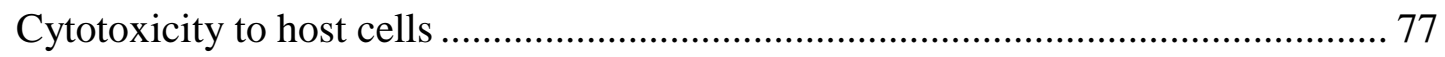

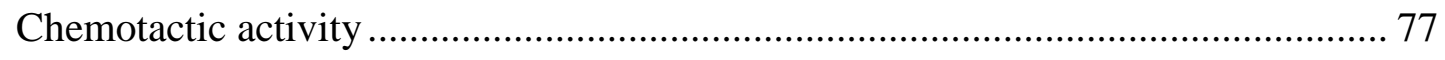


SEM observation

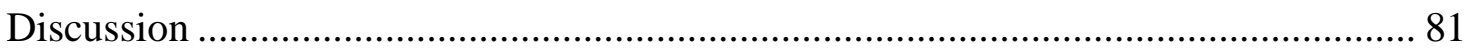

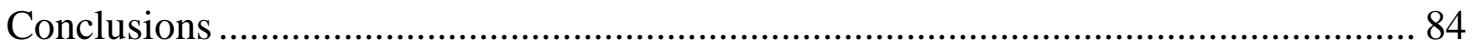

CHAPTER 4: Beta-Defensin Derived Cationic Antimicrobial Peptides with Potent Killing Activity against Gram-Negative and Gram-Positive Bacteria..................................... 85

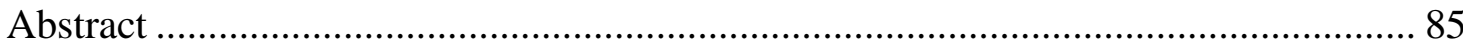

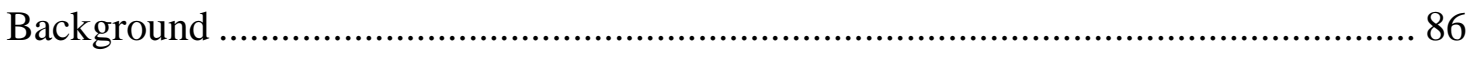

Methods and Materials .................................................................................... 88

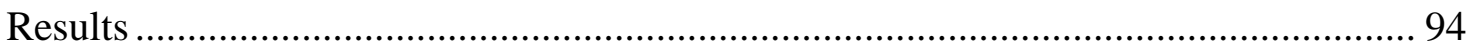

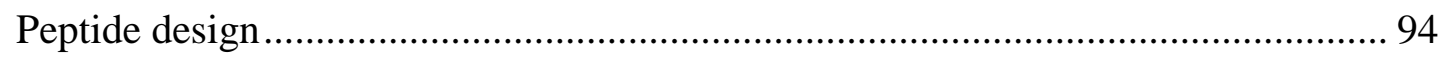

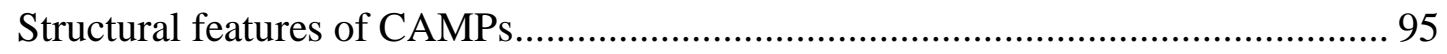

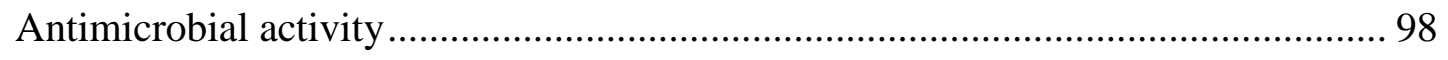

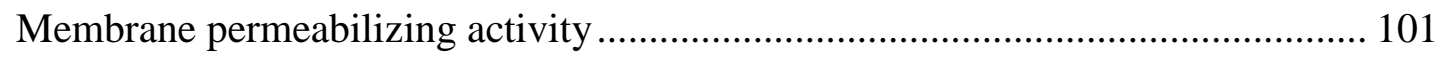

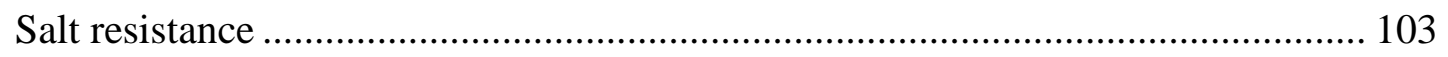

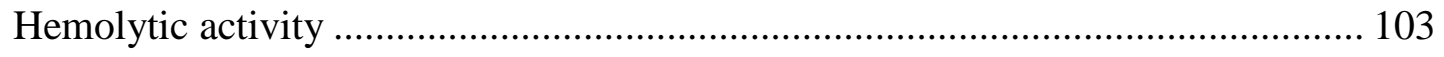

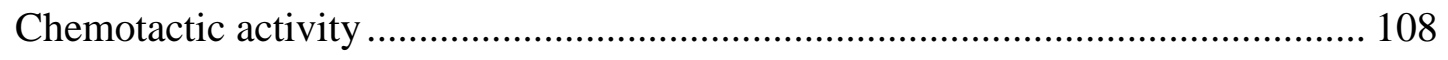

Protease resistance ...................................................................................... 110

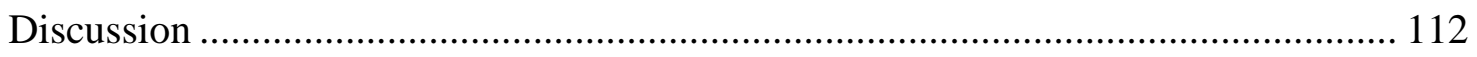

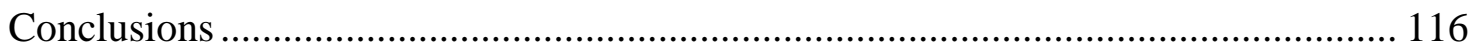


CHAPTER 5: Antimicrobial Efficacy of Selected CAMPs against P. aeruginosa Infection in a Murine Skin Wound Infection Model ................................................................. 117

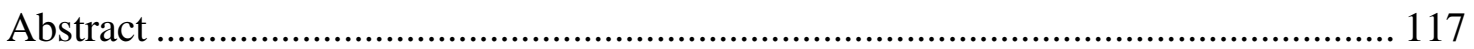

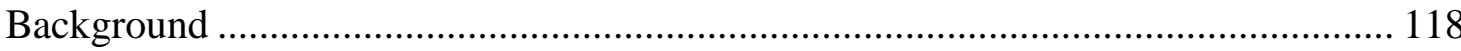

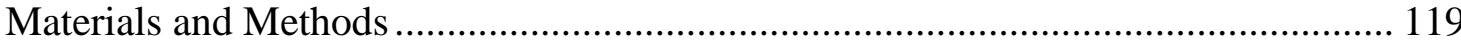

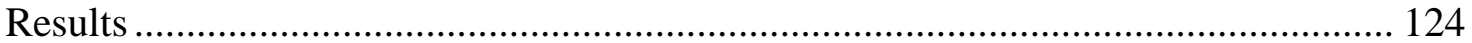

Establishment of $P$. aeruginosa infection....................................................... 124

The acute toxicity of CAMP-A and CAMP-B …............................................ 126

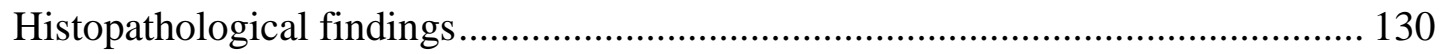

Clearance of bacterial infection by CAMP-A and CAMP-B ............................... 132

Histology of skin post-infection and treatment ................................................... 133

Efficacy of CAMP-A at higher concentrations .............................................. 137

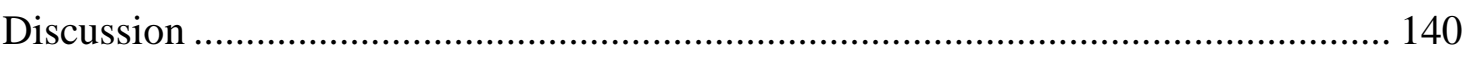

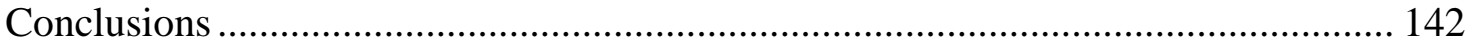

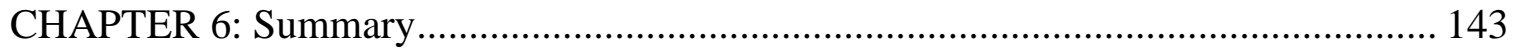

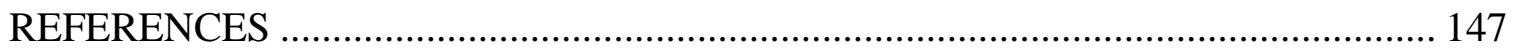

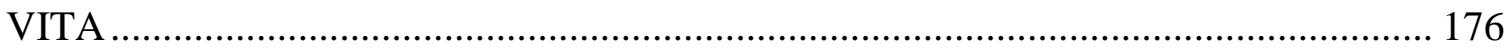




\section{LIST OF TABLES}

Table 1-1. Antimicrobial activities of avian $\beta$-defensins .............................................. 11

Table 2-1. Amino acid sequence and properties of AvBD-6 and AvBD-12 ………........ 34

Table 2-2. Minimum inhibitory concentrations of AvBD-6 and AvBD-12 .................... 34

Table 3-1. Amino acid sequences of avian $\beta$-defensin-12 (AvBD-12) analogs ............... 64

Table 3-2. The minimum inhibitory concentration and minimum bactericidal concentration

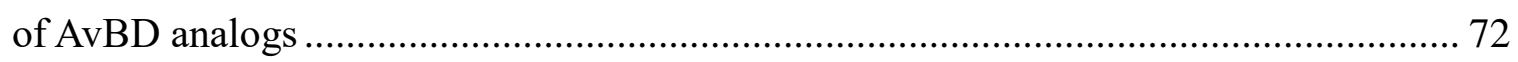

Table 4-1. The characteristics of newly designed CAMPs................................................. 97

Table 4-2. The minimum inhibitory concentration (MIC) of CAMPs ............................. 99

Table 4-3. Therapeutic index of new cationic antimicrobial peptides (CAMPs) ........... 106

Table 5-1. Effect of low doses (4×MIC) of CAMP-A and CAMP-B on the hematological

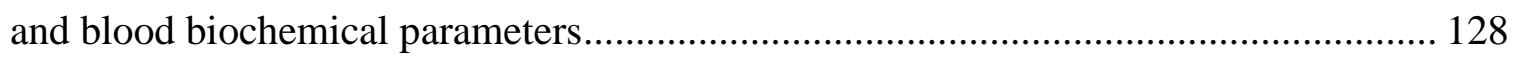

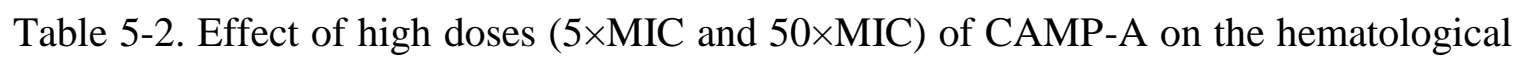
and blood biochemical parameters. 129 


\section{LIST OF FIGURES}

Figure 1-1. Structures of antimicrobial peptides..................................................... 3

Figure 1-2. Mechanisms of action of antimicrobial peptides .................................... 6

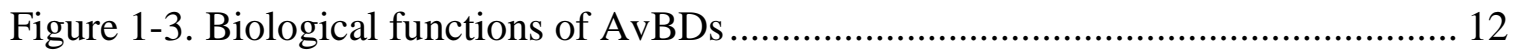

Figure 2-1. Antimicrobial activity of AvBD-6 and AvBD-12 against E. coli, S.

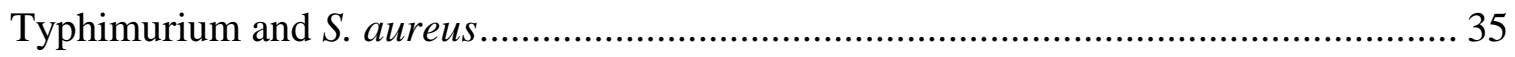

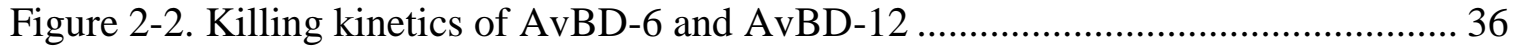

Figure 2-3. The LPS-neutralizing activity of AvBD-6 and AvBD-12 ......................... 38

Figure 2-4. Cytotoxicity of AvBD-6 and AvBD-12 on host cells ................................. 39

Figure 2-5. Expression of chicken CCR2 and CCR6 in CHO-K1 cells ........................ 41

Figure 2-6. Chemotactic activity of AvBD-6 and AvBD-12 ................................... 42

Figure 2-7. Structure analysis of reduced and wildtype AvBD-6 and -12.................... 44

Figure 2-8. Antimicrobial and chemotactic activities of reduced AvBD-6 and -12 ....... 45

Figure 2-9. The LPS-neutralizing activity of reduced AvBD-6 and AvBD-12 .............. 46

Figure 2-10. Transmission electron microscopy (TEM) analysis of AvBD-induced

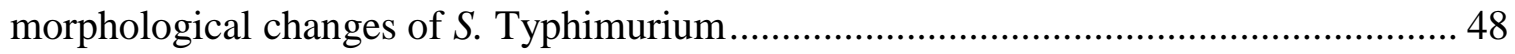

Figure 2-11. AvBD interaction with $S$. Typhimurium genomic DNA .......................... 49

Figure 3-1.The predicted three-dimensional structures of AvBD-6 and AvBD-12........ 65

Figure 3-2. The predicted $\beta 2-\beta 3$ loop in AvBD-12A2 and AvBD-12A3 ........................ 66

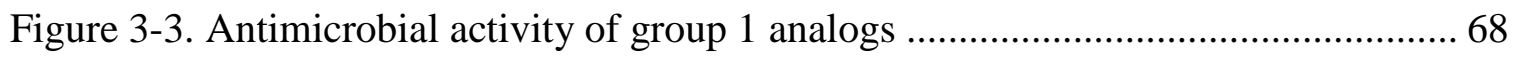

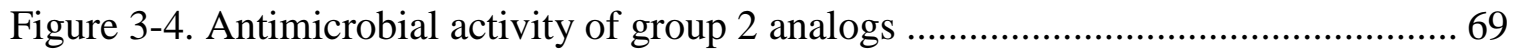

Figure 3-5. Antimicrobial activity of hybrid peptide AvBD-12/6 ............................ 70

Figure 3-6. Effect of $\mathrm{NaCl}$ on the antimicrobial activity of group one AvBDs............... 74 
Figure 3-7. Effect of $\mathrm{NaCl}$ on the antimicrobial activity of group 2 analogs 75

Figure 3-8. Effect of $\mathrm{NaCl}$ on the antimicrobial activity of analog AvBD-12/6.... 76

Figure 3-9. Chemotactic activity of AvBD-12 analogs for CCR2 transfected CHO-K1 cells and mouse immature dendritic JAWSII cells 78

Figure 3-10. Scanning electron microscopy of $S$. Typhimurium treated with AvBDs ..... 80

Figure 4-1. The predicted structures of templates CAMP-t1 and CAMP-t2 .................. 96

Figure 4-2. The far-UV CD spectra of CAMPs in $\mathrm{H}_{2} \mathrm{O}$ recorded at room temperature ... 97

Figure 4-3. Membrane permeabilizing activity of CAMPs ...................................... 102

Figure 4-4. Effect of salts on the antibacterial activity of CAMPs against P. aeruginosa

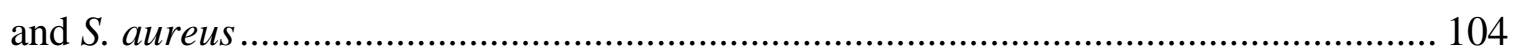

Figure 4-5. Hemolytic activity of CAMPs ............................................................ 105

Figure 4-6. Cytotoxicity of CAMPs to JAWSII and CHO-K1 cells............................. 107

Figure 4-7. Chemotactic activity of CAMPs ..................................................... 109

Figure 4-8. Effects of protease treatment on the antimicrobial activity of CAMP-A and

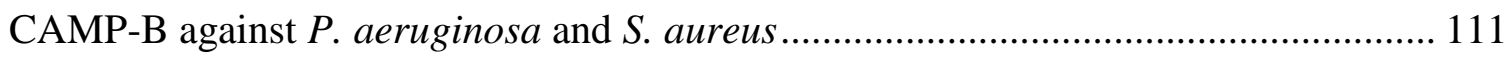

Figure 5-1. Establishment of $P$. aeruginosa infection in mice ................................. 125

Figure 5-2. Effect of CAMPs on body weight and visual inspection scores ................ 127

Figure 5-3. Histology of wounded skin following treatment with CAMPs.................. 131

Figure 5-4. Body weight and visual inspection scores of treated mice ....................... 134

Figure 5-5. The bacterial load in skin wound following treatment with CAMP-A and

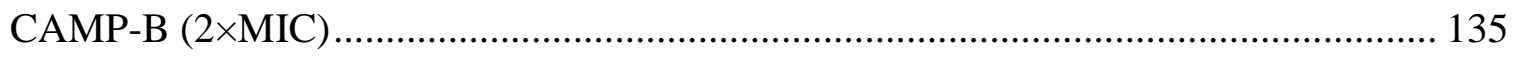

Figure 5-6. Efficacy of CAMP-A and CAMP-B (2×MIC) in prevention of P. aeruginosa

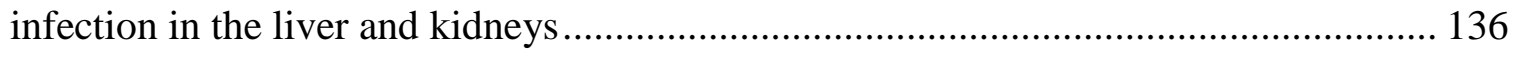


Figure 5-7. Body weight, visual inspection scores, wound closure, and inflammatory scores of mice treated with higher doses of CAMP-A ..................................................... 138 Figure 5-8. The efficacy of CAMP-A $(5 \times \mathrm{MIC}$ and $50 \times \mathrm{MIC})$ in the treatment of $P$.

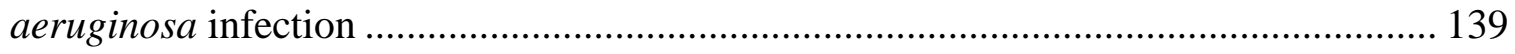




\section{LIST OF ABBREVIATIONS}

AA Amino acid

ALB Albumin

ALT Alkaline aminotransferase

AMPs Antimicrobial peptides

ANOVA One-way analysis of variance

AvBD Avian beta-defensin

BSA Bovine serum albumin

CAMP Cationic antimicrobial peptide

CD Circular dichroism

CFU Colony-forming unit

C.I. Chemotaxis index

4-CN 4-Chloro-1-Naphthol

ESI-MS Electrospray ionization mass spectrometry

FBS Fetal bovine serum

fMLF N-Formyl-methionyl-leucyl-phenylalanine

Fmoc 9-fluorenyl-methoxycarbonyl

GFP Green fluorescent protein

GGT Gamma-glutamyltransferase

GM-CSF Granulocyte macrophage-colony stimulating factor

HBD Human $\beta$-defensin

HCT Hematocrit

HDPs Host defense peptides 


\begin{tabular}{|c|c|}
\hline HGB & Hemoglobin \\
\hline HRP & Horseradish peroxidase \\
\hline Ig & Immunoglobin \\
\hline LPS & Lipopolysaccharides \\
\hline LTA & Lipoteichoic acid \\
\hline $\mathrm{MBC}$ & Minimum bactericidal concentration \\
\hline $\mathrm{MCH}$ & Mean cell hemoglobin \\
\hline $\mathrm{MCHC}$ & Mean cell hemoglobin concentration \\
\hline $\mathrm{MCV}$ & Mean cell volume \\
\hline MRSA & Methicillin-resistant Staphylococcus aureus \\
\hline MIC & Minimum inhibitory concentration \\
\hline NADPH & Nicotinamide adenine dinucleotide phosphate \\
\hline PBS & Phosphate buffered saline \\
\hline PLT & Platelet count \\
\hline $\mathrm{RBC}$ & Red blood cell \\
\hline RDW & Red cell distribution width \\
\hline RP-HPLC & Reversed-phase high-performance liquid chromatography \\
\hline SD & Standard deviation \\
\hline SEM & Scanning electron microscopy \\
\hline TEM & Transmission electron microscopy \\
\hline TLR4 & Toll-like receptor 4 \\
\hline TSA & Trypticase soy agar \\
\hline WBC & White blood cell \\
\hline
\end{tabular}




\begin{abstract}
The increasing prevalence of antibiotic-resistance and lack of effective antibiotics pose a serious threat to animal health and public health. Host antimicrobial peptides (AMPs) show broad-spectrum antimicrobial activity against various microbes with low potential for resistance development, compared to conventional antibiotics, indicating great potentials as therapeutic agents. Despite such promise, several limitations hinder the application of AMPs in the clinic, including high manufacturing cost, cytotoxicity, and stability in physiological conditions. New strategies are needed to solve those problems for their application.

Avian beta-defensins (AvBD) are small, cationic, antimicrobial peptides. The potential application of AvBDs as antibiotic alternatives against antibiotic-resistant and zoonotic bacterial pathogens has been the subject of interest. In the first study, the biological functions of two AvBDs, AvBD-6 and AvBD-12, were determined under various experimental conditions. The results showed that AvBD-6 (+7) was more potent than AvBD-12 (+1) against E. coli, S. Typhimurium, and S. aureus as well as clinical isolates of extended-spectrum beta-lactamase (ESBL)-producing E. coli and K. pneumoniae. The antibacterial activity of AvBDs was greatly compromised under physiological salt concentrations. Both AvBDs demonstrated mild chemotactic property for chicken macrophages and AvBD-12, at relatively high concentrations, could also induce the migration of murine immature dendritic cells. The chemotactic property required the presence of chemokine receptor 2 (CCR2) on host cells and the conserved disulfide bridges of the peptides. The two AvBDs were nontoxic to $\mathrm{CHO}-\mathrm{K} 1$, macrophages, or immature dendritic cells.
\end{abstract}


In the second study, the structure-function relationship of AvBDs was characterized by evaluating the biological functions of seven newly designed AvBD analogs which possessed different net positive charges (+1 to +9 ), hydrophobicity (33\% to $53 \%$ ), and number of disulfide bridges ( 0 to 3 ). The results indicated that the net positive charge and charge distribution determined the antimicrobial potency of AvBDs whereas the three conserved disulfide bridges were essential to the chemotactic property and the maximum antimicrobial activity of AvBDs. All analogs were nontoxic to host cells, but were still sensitive to salts. It was noteworthy that analog AvBD-12A3 with a high net positive charge of +9 , a moderate degree of hydrophobicity of $40 \%$ and a CCR2-binding domain showed the most potent antimicrobial activity and retained $50 \%$ of the chemotactic activity of wild-type AvBD-12. These properties suggested that AvBD-12A3 might serve as a template for the design of novel AMPs.

In the third study, an integrated approach was used to design to cationic antimicrobial peptides (CAMP) based on the key functional domains of AvBD-6 and AvBD-12 as well as commonly desirable features of antimicrobial peptides, such as structurally simple, resistant to proteases and salts, potent antimicrobial activity, and potential chemotactic property. Of the four newly designed peptides, CAMP-t1 contained the CCR2 binding domain (N-terminal loop and adjacent $\alpha$-helix) of AvBD-12 while CAMP-t2 was comprised of the key amino acid residues contributing to the concentrated positive surface charge and hydrophobicity of AvBD-6. CAMP-t2 was further optimized to yield two $\alpha$ helical peptides CAMP-A and CAMP-B by avoiding potential protease cutting sites, incorporating a C-terminal poly-Trp tail, $\mathrm{N}$-terminal acetylation, and $\mathrm{C}$-terminal amidation. The results showed that the antimicrobial potency of the four peptides against $P$. 
aeruginosa, S. aureus and methicillin-resistant $S$. pseudintermedius strains (MRSP) could be ranked as CAMP-A > CAMP-B > CAMP-t1 and CAMP-t2. CAMP-A and CAMP-B also demonstrated promising characteristics as therapeutic agents, such as rapid membrane attacking (30 min), resistance to physiological concentrations of $\mathrm{NaCl}$ and $\mathrm{CaCl}_{2}$, tolerance to major proteases, and a moderately high therapeutic index of $\geq 4.5$ which suggested that the peptide is potentially safe for clinical use. Finally, the efficacy and safety of CAMP-A and CAMP-B for treatment of $P$. aeruginosa infection were assessed using a murine skin wound model. Treatment with CAMP-A and CAMP-B significantly reduced bacterial load in the infected wound, compared to untreated control group. CAMP-A completely prevented the dissemination of $P$. aeruginosa from infected wounds to systemic organs (liver and kidney). CAMP-B prevented the spread of $P$. aeruginosa from infected wounds to systemic organs in four of the six mice (67\%). The treatment efficacy of CAMP-A, in terms of wound bacterial clearance, was dose- and time-dependent. CAMP-A and CAMPB did not show obvious toxicity to mice, except that CAMP-A ( $\geq 5 \mathrm{x}$ MIC) was associated with high ALT level in two of the twelve mice.

In conclusion, the present dissertation research has not only characterized the biological functions of AvBDs and their structure-function relationship, but also established an integrated CAMP design strategy. The end products, CAMP-A and CAMP-B, can be further developed as effective therapeutic or preventive agents. 


\section{CHAPTER 1: Avian Beta-Defensins: Potent Bactericidal Agents}

\section{Host Antimicrobial Peptides (AMPs)}

The Centers for Disease Control and Prevention (CDC) reported that over 2 million cases of antibiotic-resistant bacterial infections occurred each year in the United States, resulting in more than 23,000 deaths (https://www.cdc.gov/). Above $70 \%$ of the bacteria causing infections were resistant to at least one of the commonly prescribed antibiotics [1]. Due to increased prevalence of antibiotic-resistant bacteria and lack of new and effective antibiotics for treatment, antimicrobial peptides as potential therapeutic agents have been extensively studied $[2,3]$.

Host antimicrobial peptides (AMPs), also known as host defense peptides (HDPs), have been found in vertebrates, invertebrates, and plants [4]. To date, more than 2600 natural AMPs can be found in the antimicrobial peptide database (APD) [5, 6], including 123 from humans, 1972 from animals, 321 from plants, and 287 from insects. The first reported animal AMP is bactericidal phagocytin which was isolated from rabbit leukocytes in 1956 [7]. Most AMPs are cationic and amphipathic peptides [8], consisting less than 100 amino acid residues [9]. They are constitutively or inducibly expressed in certain tissues and organs which are constantly exposed to microbial pathogens [10], such as epithelial cells of skin, gastrointestinal, and respiratory tracts. The cationic AMPs have broad-spectrum antimicrobial activities against bacteria, fungi, protozoan, viruses, and even cancer cells $[11,12]$. In addition to cationic AMPs, there are several anionic AMPs found in sweat glands, bronchoalveolar lavage fluid, and skin, which are rich in glutamic and/or aspartic acids, such as amphibian Maximin H5 [13] and human Dermcidin [14]. Compared to cationic AMPs, anionic AMPs are relatively narrow-spectrum antimicrobial agents or need 
additional molecules for their antimicrobial activity. For example, Maximin H5 showed bactericidal activity only against Staphylococcus aureus (S. aureus), but not other bacteria (Bacillus pyocyaneus and Escherichia coli) and fungi (Candida albicans and Aspergillus flavus) [13]. An ovine pulmonary surfactant-associated anionic peptide was only active against Pasteurella haemolytica in the presence of zinc ions [15], and Dermcidin, a human antimicrobial peptide secreted by sweat glands, was more active against $S$. aureus than $E$. coli in sweat $\mathrm{pH} 4$ to 6.8 [14].

This review focuses primarily on the structural characteristics of cationic AMPs and their diverse mechanisms of action, with a special emphasis on avian $\beta$-defensins (AvBDs) and the strategies to overcome the drawbacks of natural AvBDs as therapeutic agents.

\section{Classification of AMPs}

Although variable in length, amino acid composition, and host origin, AMPs can be classified into the following four types based on their secondary structures [16]: linear or extended, $\alpha$-helix, circular loop, and $\beta$-sheet (Figure 1-1). The $\alpha$-helix and $\beta$-sheet structures are more frequently seen in natural peptides and their synthetic analogs. The common characteristics of these AMPs are cationic and amphipathic which account for their ability to interact with negatively charged bacterial cell wall components and amphipathic cell membranes [17]. 
(A)

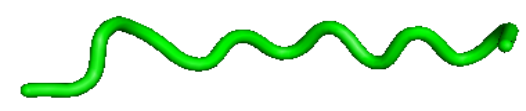

(C)

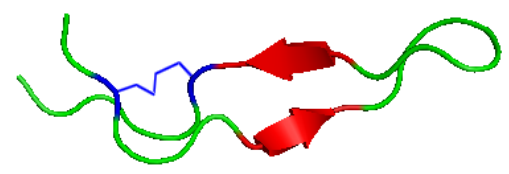

(B)

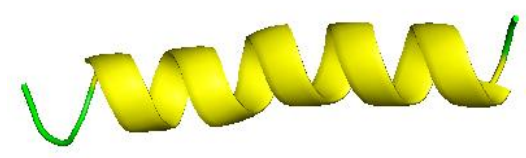

(D)

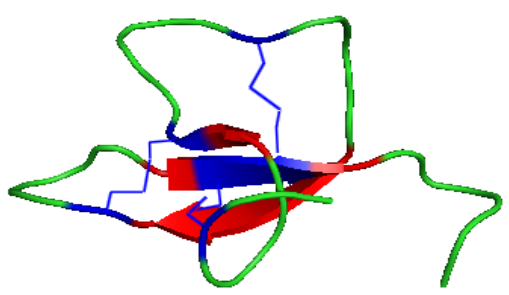

Figure 1-1. Structures of antimicrobial peptides

(A) linear (extended) (e. g. bovine indolicidin, PDB code 1G8C) [18]; (B) $\alpha$-helical (e. g. xenopus magainin 2, PDB code 2MAG) [19]; (C) loop (e. g. bovine lactoferricin B, PDB code 1LFC) [20]; (D) $\beta$-sheet (e.g. avian $\beta$-defensin 2, PDB code 2LG5) [21]. Helix: yellow, $\beta$-sheet: red. Disulfide bonds are indicated in blue color. Images were prepared with PyMOL (https://pymol.org). 


\section{Mechanisms of Action}

The antimicrobial action of cationic AMPs can be categorized into two main mechanisms: 1) disrupting membrane integrity via interacting with negatively charged cell wall and/or membrane components, 2) interfering the synthesis and function of intracellular components such as DNA, RNA, and proteins [17]. The initial binding of AMPs to the microbial membrane is a prerequisite for membrane disruption and interference of intracellular components, so most AMPs utilize dual killing mechanisms to achieve their antimicrobial potential [22]. For instance, AvBD103b, an avian defensin isolated from king penguin, can penetrate bacterial membrane and intercalate into genomic DNA of Salmonella enterica serovar Enteritidis ( . Enteritidis), ultimately causing cell death [22]. AMPs with different mechanisms of action have been proposed as alternative therapeutic agents for the treatment of microbial infections, especially infections involving multidrugresistant microbial strains [23].

\subsection{Membrane-Active AMPs}

The classical mechanism of action of AMPs involves their ability to damage microbial membrane, since the integrity of membrane prevents cell lysis due to the high cytoplasmic osmotic pressure [24]. Many membrane-active AMPs are amphipathic, including both hydrophilic (comprised mostly of cationic residues) and hydrophobic faces [17]. The positive charge ensures the initial electrostatic interaction of AMPs with the negatively charged cell wall components, such as lipopolysaccharide (LPS) and lipoteichoic acid (LTA) [25], while the hydrophobic residues enable the AMPs to insert into the bacterial membrane to form pores [26]. The action of AMPs on microbial membrane bilayers are determined by their amino acid residues, amphipathicity, charge, and length, and pore- 
formation can be further divided into three types: barrel-stave, carpet, or toroidal model $[27,28]$. Figure $1-2$ outlines the above modes of action of AMPs.

Dysregulation of the bacterial membrane permeability by AMPs results in interruption of osmotic pressure and a leakage of cytoplasm, thereby causing cell death. In contrast to prokaryotic cell membranes, mammalian cytoplasmic membranes are composed of neutral zwitterionic phospholipids, such as phosphatidylethanolamine (PE), phosphatidylcholine (PC) or sphingomyelin (SM), and lack negatively charged lipids in the outer layer of the membrane [29]. Moreover, the presence of cholesterol in mammalian cell membranes inhibits the activity of AMPs by stabilizing the lipid bilayer or directly interacting with AMPs [30]. The difference in membrane components between host cells and bacteria confers the selective targeting on microbes by AMPs. Hence, boosting the interaction of AMPs with microbial membrane should be considered when design and modify AMPs for stronger antimicrobial activity.

\subsection{Intracellularly Active AMPs}

AMPs can traverse bacterial membranes and interact with DNA, RNA, and proteins to inhibit their synthesis, thereby disrupting normal cell function $[8,12,31,32]$. Majority of bacterial proteins are membrane-associated proteins, which play important roles in bacterial physiology including nutrient or proton transport, energy production, and cell-tocell communication $[17,33]$. The function of these molecules can be altered by AMPs even without cell lysis. For example, buforin II, a toad cationic, linear AMP with 21 amino acid residues, can cross E. coli cell membranes and binds to intracellular DNA and RNA without disrupting the membranes [34]. 


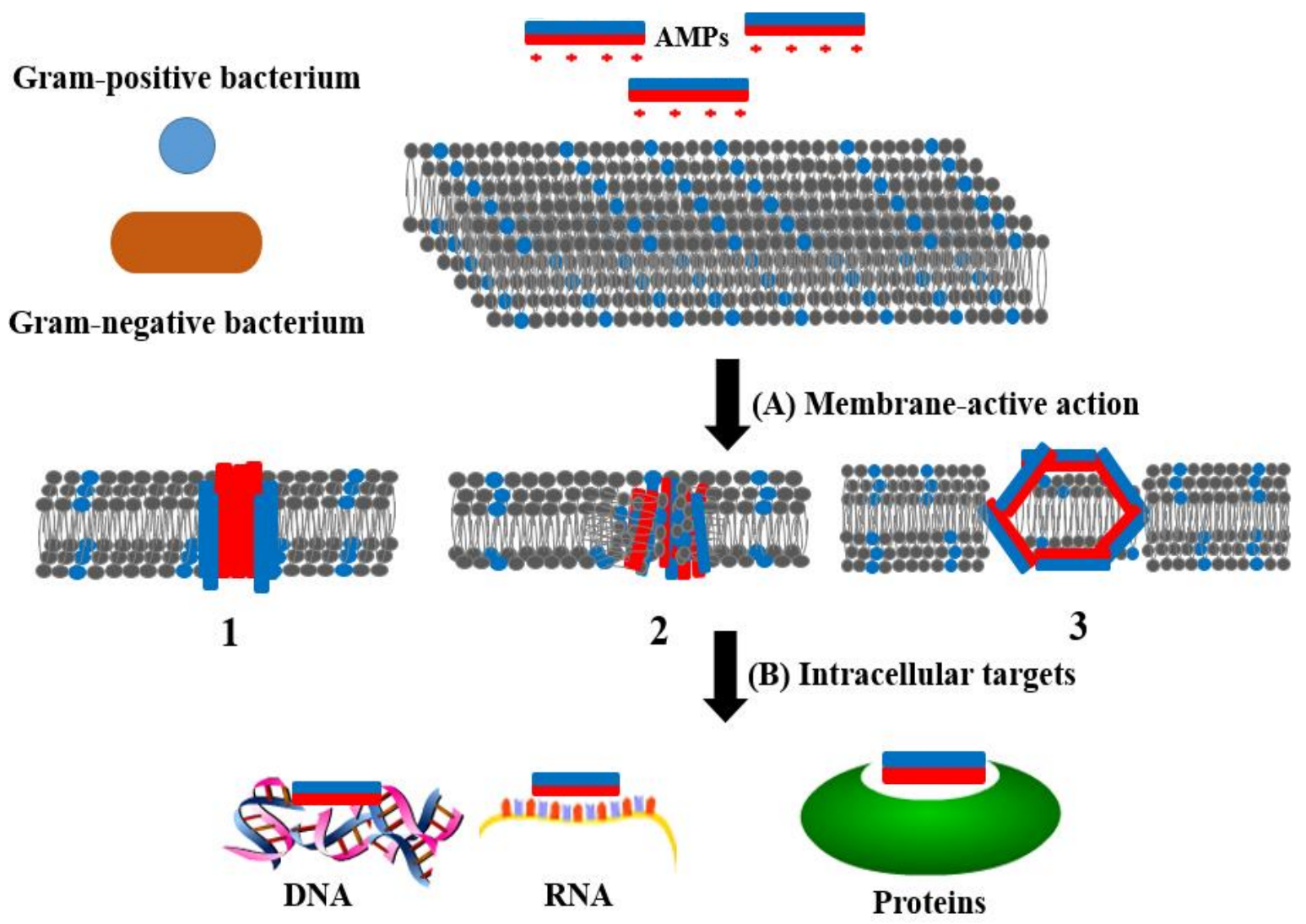

Figure 1-2. Mechanisms of action of antimicrobial peptides

(A) Membrane-active action. First, AMPs electrostatically interact with bacterial bilayer membrane ( $\mathrm{O}$, blue lipid heads are negatively charged). Then they interrupt the integrity of membrane by forming different pores [35] as following models. (1) Barrel-Stave model: AMP molecules perpendicularly insert into the membrane. (2) Toroidal pore model: AMPs bind with phospholipid head groups of the membrane. (3) Carpet model: Parts of the membrane are coated with AMP molecules. (B) Intracellular targets. AMPs can penetrate the membrane bilayers and interact with intracellular molecules, such as DNA, RNA, and proteins [17]. 
Another example is PR-39 from swine intestine, and it kills E. coli in a non-lytic mode by inhibiting protein and DNA synthesis [36]. Currently, two major mechanisms of AMP translocation across microbial membrane has been proposed, including direct penetration or endocytosis. However, the details of the molecular uptake remain to be discovered [26].

\section{Avian $\beta$-defensin (AvBDs) and Their Biological Functions}

Defensins, a major group of AMPs, contain six highly conserved cysteine residues that form three intramolecular disulfide bonds. On the basis of the cysteine pairing pattern, they can be subdivided into three subfamilies, $\alpha-, \beta$-, and $\theta$-defensins [37]. The cysteine bridges between $\mathrm{Cys}^{1}-\mathrm{Cys}^{6}, \mathrm{Cys}^{2}-\mathrm{Cys}^{4}, \mathrm{Cys}^{3}-\mathrm{Cys}^{5}$ for $\alpha$-defensins, and versus $\mathrm{Cys}^{1}{ }^{-} \mathrm{Cys}^{5}, \mathrm{Cys}^{2}-$ $\mathrm{Cys}^{4}, \mathrm{Cys}^{3}$-Cys ${ }^{6}$ for $\beta$-defensins [38]. The $\theta$-defensins are cyclic defensins originally found in rhesus monkeys with cysteine bridges between $\mathrm{Cys}^{1}-\mathrm{Cys}^{6}, \mathrm{Cys}^{2}-\mathrm{Cys}^{5}, \mathrm{Cys}^{3}-\mathrm{Cys}^{4}$ [39]. In avian species, only $\beta$-defensin has been found. Totally, 14 AvBD genes have been identified in chicken genome [40]. AvBDs have well-defined $\beta$-sheet or $\beta$-sheet plus $\alpha$ helix structures [21, 41]. The three-dimensional nuclear magnetic resonance (NMR) structure of king penguin avian $\beta$-defensin 103b (AvBD-103b) displays the disulfide bridge motif and a three-stranded $\beta$-sheet as characteristics of $\beta$-defensins and a short $\alpha$-helix at the N-terminus [42]. In contrast, the three-dimensional NMR structures of chicken AvBD2 (Figure 1-1) and AvBD7 only display the three-stranded $\beta$-sheet without the helix [21, 43].

The first two avian defensins (AvBD-1 and AvBD-2) were isolated from leukocytes or bone marrow extracts and referred to as Gallinacins 1-2 [44]. AvBDs are expressed in skin (such as AvBD-3, -9, and -11), respiratory tract (such as AvBD-1 to -3, -6, -9 and -10), urogenital tract (AvBD-1 to-3, -4, -6, -7 and -10), and digestive tract (AvBD-2, -3, -5, -8 
and -9) of chickens [45]. Most AvBDs genes are expressed in a wide range of tissues, for examples, AvBD-1 and AvBD-3 [46]. While some AvBD genes are constitutively expressed, others are activated by exposure to microbial pathogens, such as Salmonella Typhimurium (S. Typhimurium) [47] and Clostridium perfringens (C. perfringens) [48], as well as feed supplements (e. g. vitamin $\left.\mathrm{D}_{3}\right)$ [49].

The avian heterophils lack myeloperoxidases and depend primarily on nonoxidative mechanisms for antimicrobial activity, including cationic defensins [50]. Therefore, AvBDs play more important roles in innate immunity against bacteria, viruses, fungi, parasites [51, 52]. Evidence has also become available that AvBDs can regulate various adaptive immune responses, such as chemotaxis and inducing or suppressing inflammatory responses [53].

\subsection{Antimicrobial Activity}

Most AvBDs that have been subjected to functional characterizations, show antimicrobial activity against different bacterial and fungal species (Table 1). The newly discovered AvBD-14 is highly expressed in many tissues and its antimicrobial activity against $E$. coli has been verified [54]. The mechanism of action is thought to be similar to other cationic AMPs, which utilize their positively charged residues to interact with negatively charged membrane components and hydrophobic residues to help insert the peptides into microbial membrane, which cause membrane disruption and eventually microbial death $[45,55,56]$. This model highlights the importance of amphipathic and cationic properties of AMPs in killing microbial pathogens. The importance of net positive charge on antibacterial activity has been demonstrated via the study of biological functions of AvBD-2, -6 and -12 [57]. AvBD-12 with the lowest net charge $(+1)$ showed the lower antibacterial activity than 
AvBD-2 (+4) and -6 (+7) against Gram-negative and Gram-positive bacteria [57]. In another study, AvBD2 (+4), was shown to be less active than AvBDs with higher charge (AvBD-7, +6, and AvBD-1, +8) in killing Gram-negative bacteria [58].

The antimicrobial activity of AvBDs such as chicken AvBD-6, AvBD-9, and AvBD-12, is inhibited by cationic salts at physiological concentration (e. g. $150 \mathrm{mM} \mathrm{NaCl}$ or $2 \mathrm{mM}$ $\left.\mathrm{CaCl}_{2}\right)[45,55,59]$. This phenomenon, referred to as salt sensitivity, was also shown by other host defensins such as mouse $\beta$-defensin 1 (mBD-1) [60] and human $\beta$-defensin 2 (hBD-2) [61]. The antimicrobial or antiseptic activity of AvBDs could also be achieved through their LPS-neutralizing function. Data from our studies revealed that AvBDs neutralized LPS activity in a dose-dependent manner and the LPS-neutralizing function was not affected by $\mathrm{NaCl}$ concentration, ranging from $0 \mathrm{mM}$ to $137 \mathrm{mM}$ [59]. The antimicrobial activity of chicken AvBD-9, penguin AvBD-103b, and duck AvBD-2 maintained over a broad temperature and $\mathrm{pH}$ range [55]. For example, post-incubation 30 min in conditions of temperature from -20 to $100{ }^{\circ} \mathrm{C}$ or $\mathrm{pH}$ from 3 to 12 , duck AvBD-2 still retained its antimicrobial activity against $S$. aureus [62].

\subsection{Immunomodulatory activity of AvBDs}

In addition to their antimicrobial activity, AvBDs possess immunomodulatory properties that have been observed with mammalian $\beta$-defensins [63, 64]. Recombinant duck Apl_AvBD-2 expressed in HEK-293T cells induced the migration of duck spleen B- and T-cells in a concentration-dependent manner and the migration could be blocked by antiduck AvBD2 polyclonal antibodies [65, 66]. Incubation of chicken peripheral blood lymphocytes with AvBD-13 at low concentration of $0.625-1.25 \mu \mathrm{g} / \mathrm{ml}$ along with $5 \mu \mathrm{g} / \mathrm{ml}$ of lipopolysaccharide (LPS) and $20 \mu \mathrm{g} / \mathrm{ml}$ of concanavalin A (ConA) enhanced the 
proliferation of lymphocytes, compared to incubation with LPS and ConA alone [67]. In this study, given AvBD-13 (1 $\mu \mathrm{g} / \mathrm{ml})$ in drinking water could enhance immunoglobulin (Ig) $\mathrm{G}$ at the age of 4 to 10 days and $\operatorname{IgM}$ at the age of 4 and 10 days [67]. Data from our own studies indicated that both AvBD-6 and AvBD-12 were chemoattractants of chicken macrophages (MQNC-SU), mouse immature dendritic cell line (JAWSII, AvBD-12 only), and Chinese hamster ovary (CHO) cells expressing CCR2 [59]. Overall, those studies have provided strong evidence of AvBDs linking the innate and adaptive immune systems in response to microbial infections. The biological functions of AvBDs have been summarized in Figure 1-3. 
Table 1-1. Antimicrobial activities of avian $\beta$-defensins

\begin{tabular}{|c|c|c|c|c|c|c|c|c|c|c|c|c|c|c|}
\hline Microorganisms & & 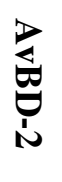 & 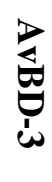 & 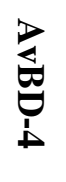 & 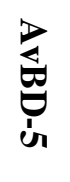 & 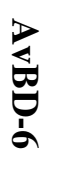 & t) & $\underset{\infty}{\stackrel{D}{\infty}}$ & 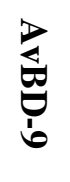 & 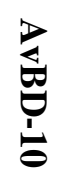 & 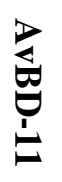 & 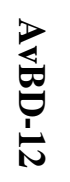 & 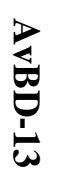 & 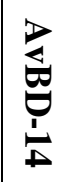 \\
\hline \multicolumn{15}{|l|}{ Gram-negative bacteria } \\
\hline Escherichia coli & $\checkmark$ & $\checkmark$ & $\checkmark$ & $\checkmark$ & & $\checkmark$ & $\checkmark$ & & $\checkmark$ & $\checkmark$ & $\checkmark$ & $\checkmark$ & $\checkmark$ & $\checkmark$ \\
\hline Salmonella Enteriditis & $\checkmark$ & & & & & & & & & & & & & \\
\hline Salmonella & $\checkmark$ & $\checkmark$ & & $\checkmark$ & & $\checkmark$ & $\checkmark$ & & $\checkmark$ & $\checkmark$ & & $\checkmark$ & $x$ & \\
\hline Pasteurella multocida & $\checkmark$ & & & & & & & & & & & & & \\
\hline Campylobacter jejuni & $\checkmark$ & & & & & & & & $\checkmark$ & & & & & \\
\hline Bordetella avium & $\checkmark$ & & & & & & & & & & & & & \\
\hline Klebsiella pneumonia & & & & $\checkmark$ & & & & & $\checkmark$ & $\checkmark$ & & & & \\
\hline Pseudomonas & & & & $\checkmark$ & & $\checkmark$ & $\checkmark$ & & $\checkmark$ & $\checkmark$ & & $\checkmark$ & & \\
\hline Shigella sonneri & & & & $\checkmark$ & & & & & $\checkmark$ & $\checkmark$ & & & & \\
\hline \multicolumn{15}{|l|}{ Gram-positive bacteria } \\
\hline Listeria monocytogenes & $\checkmark$ & $\checkmark$ & & & & & $\checkmark$ & & & & & & $x$ & \\
\hline Staphylococcus aureus & $\checkmark$ & $\checkmark$ & & $\checkmark$ & & $\checkmark$ & $\checkmark$ & & $\checkmark$ & $\checkmark$ & & $\checkmark$ & $x$ & \\
\hline \multicolumn{15}{|l|}{ Staphylococcus } \\
\hline Staphylococcus & & & & $\checkmark$ & & & & & $\checkmark$ & $\checkmark$ & & & & \\
\hline Streptococcus pyogenes & & & & & & & & & $\checkmark$ & & & & & \\
\hline Streptococcus salivarius & & & & & & & $\checkmark$ & & & & & & & \\
\hline Clostridium perfringens & & & & & & & & & $\checkmark$ & & & & & \\
\hline Enterococcus faecalis & & & & $\checkmark$ & & & & & $\checkmark$ & $\checkmark$ & & & & \\
\hline Bacillus cereus & & $\checkmark$ & & & & & $\checkmark$ & & & & & & & \\
\hline \multicolumn{15}{|l|}{ Mycoplasma } \\
\hline Mycoplasma & $\checkmark$ & & & & & & & & & & & & & \\
\hline \multicolumn{15}{|l|}{ Fungi } \\
\hline Candida albicans & $\checkmark$ & $x$ & & $\checkmark$ & & & & & $\checkmark$ & $\checkmark$ & $\checkmark$ & & & \\
\hline Neurospora crassa & & & & & & & & & $\checkmark$ & & & & & \\
\hline Aspergillus fumigatus & & & & & & & & & $\checkmark$ & & & & & \\
\hline \multicolumn{15}{|l|}{ Virus } \\
\hline Bronchitis Virus & $x$ & & & & & & & & & & & & & \\
\hline
\end{tabular}

$\checkmark$ : antimicrobial activity, $\times$ : without activity, blank: not tested. Data in this table are extrapolated from previous review papers $[45,55]$ and with updates based on studies between 2013 to 2017 [43, 52, 54, 57, 59, 68-71]. 


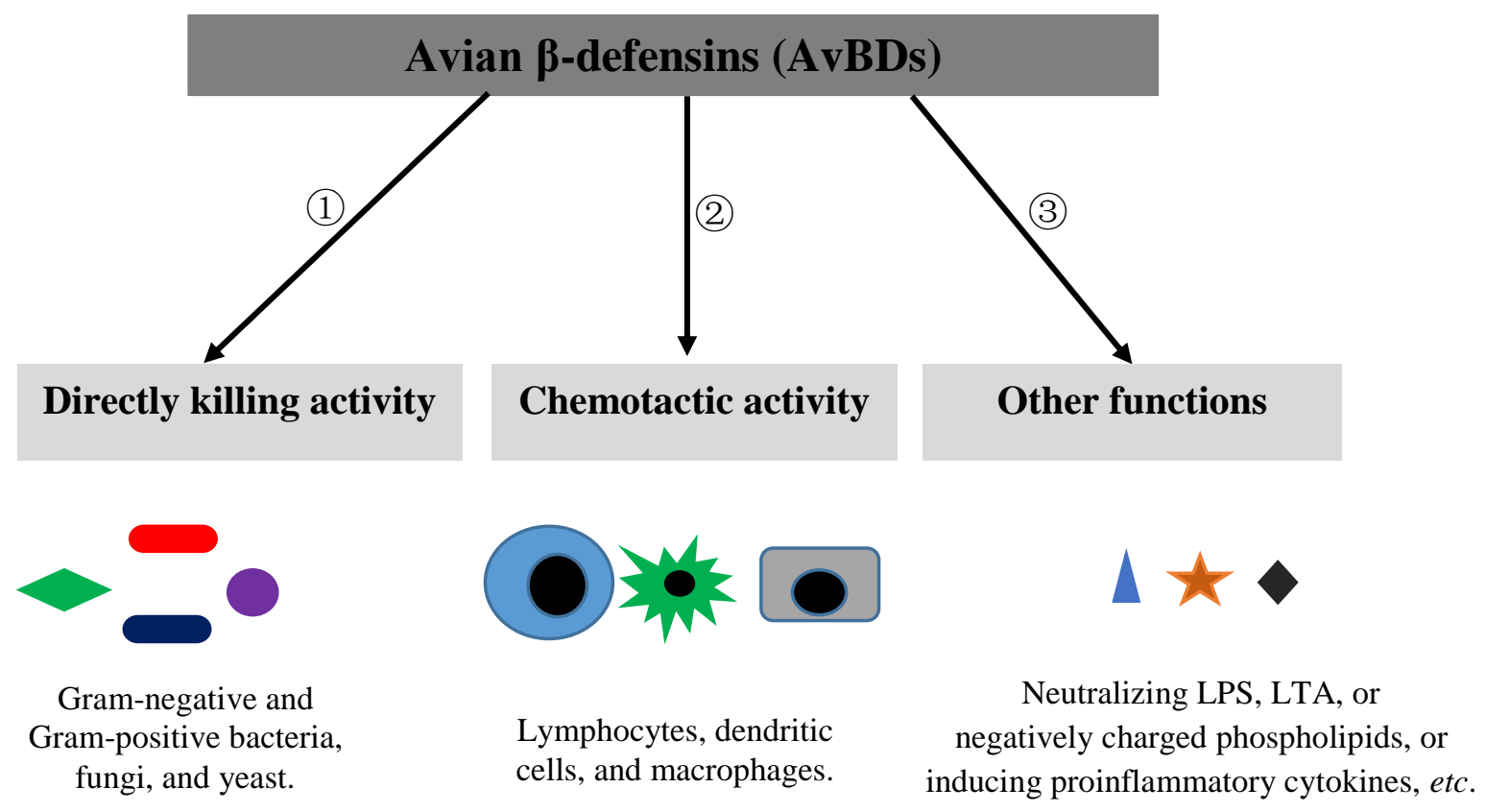

Figure 1-3. Biological functions of AvBDs

AvBDs can directly (1) and indirectly (2 and 3) combat microbial infections through their biological functions. LPS: lipopolysaccharide, LTA: lipoteichoic acid. 


\subsection{AvBDs as Therapeutic Agents}

The emergence and dissemination of multi-drug resistant (MDR) bacteria pose a great threat to public health, which calls for antimicrobial stewardship as well as the development of novel therapeutic agents with low potential for inducing antimicrobial resistance [72]. AMPs, including AvBDs, have been considered as potential therapeutic candidates because of their dual actions on the membrane and intracellular targets which differ from the mechanisms of action of currently available antibiotics. Furthermore, the LPS-neutralizing activity is an additional value that can potentially curb septic shock caused by Gram-negative bacteria. Owing to the unique antimicrobial and immune modulating properties, AvBDs may also be used as novel food preservatives [73] and vaccine adjuvants [74]. However, the application of cationic AMPs is hindered by several obstacles, including the lack of activity at physiological concentrations of cationic salts, peptide instability, and high cost associated with producing functionally active natural or recombinant peptides. For example, the antimicrobial activity of AvBD-9 against $S$. Typhimurium and $E$. coli is significantly inhibited by increasing concentration of sodium chloride from $20 \mathrm{mM}$ to $150 \mathrm{mM}$ (physiological concentration) [75]. In addition, the three intramolecular disulfide bonds must be correctly formed in order to achieve optimal antimicrobial and chemotactic activities of AvBD-12 [56]. The complicated structure (three intracellular disulfide bonds) and relatively long length of AvBDs (more than 36 amino acid residues) are associated with high production cost [9].

\section{Strategies for Developing Novel AMPs}

\subsection{Determining the structural basis of biological functions}


Understanding the structure-function relationship is the key to identifying relevant functional domains and biological features of AMPs. Data from numerous studies suggest that several properties, including peptide length, net positive charge, hydrophobicity, amphipathicity, and intracellular disulfide bonds, collectively determine the antimicrobial activity as well as the undesirable cytotoxicity of AMPs $[51,76]$. The length of an AMP can influence the mode of action, cytotoxicity, and even salt-sensitivity. In the Barrel-Stave model, $\alpha$-helical AMPs with at least 22 amino acids can transverse bacterial lipid bilayers [77]. A short melittin analog lacking 15 residues at the C-terminus exhibited higher antibacterial activity and lower toxicity to rat erythrocytes than that of the wild-type [78]. An N-terminal deletion ( 3 residues) of human $\beta$-defensin 3 (hBD-3) improved the peptide's salt-resistance [79]. The net charge of AMPs determines the electrostatic interaction of cationic AMPs with negatively charged microbial membrane components. Increasing the net positive charge of an AMP generally improves the antimicrobial activity, however, the distribution or the position of positively charged residues also determines the bactericidal activity of AMPs $[56,80]$. Hydrophobicity is another critical factor that contributes to the antimicrobial activity and selectivity (cytotoxicity to bacteria vs host cells) of AMPs [81]. For example, magainin 2 analogs with increased hydrophobicity demonstrated improved killing activity against Gram-negative bacteria as well as increased hemolytic activity [82]. It is generally agreed that amphipathicity is important to the membrane-active peptides, especially for membrane pore formation [8]. We and other researchers have illustrated disulfide bonds are necessary for chemotactic activity, which is either abolished or reduced in absence disulfide bonds, and for maximizing the antimicrobial activity of AMPs by exposure of net positive charged residues on the tertiary structure [59, 64]. 
It is noteworthy that abovementioned parameters are intrinsically related to each other and no single characteristic of AMPs is totally separable from others. Alteration of a single amino acid residue could potentially affect the structure or function of an AMP [17]. Thus, a comprehensive approach must be taken to maximize the desired biological functions and minimize cytotoxicity or any other unfavorable properties.

\subsection{Designing and modifying AMPs}

\subsubsection{Simplifying the structures of AMPs}

Short AMPs derived from natural host peptides have been explored as candidate therapeutic agents for structural simplicity and low probability of undesirable immunogenicity $[83,84]$. Simplifying the structure of AMPs not only reduces the cost of synthesis but may also improve the antimicrobial function. For example, GLI13 derived from a C-terminal truncation of AvBD-4 showed increased antimicrobial activity via targeting the cytoplasmic membrane of bacteria (e. g. E. coli and S. aureus) [85]. Several truncated AMPs (e. g. 4 residues truncated from hBD3, hBD3 $\Delta 4$ ) were more resistant to cationic salts and more effective against bacteria than their parent peptides $[79,86]$. The bactericidal activity against $E$. coli of hBD3 was unaffected in the absence of any disulfide bridge $[56,64]$. A linear analog of AVBD-12, AvBD-12A3 with an increased net positive charge of +9 and hydrophobicity of $40 \%$, showed improved antimicrobial activity against both Gram-negative and Gram-positive bacteria, compared to wild-type AvBD-12 with three disulfide bonds $[56,64]$.

\subsubsection{Alteration of Amino Acid Composition}

Individual amino acid residues play important roles in the antimicrobial activity, cytotoxicity, and selectivity of AMPs. The key residues include arginine (Arg), lysine (Lys), 
proline (Pro), tryptophan (Trp), D-amino acid residues, and unnatural amino acid residues [87, 88]. As such, alteration of amino acid composition has been a major approach to optimizing the antimicrobial activity, stability, and cytotoxicity of AMPs [56, 89-91]. For example, peptide EFK17, introduction of four Trp residues at the known protease cleavage sites in human cathelicidin antimicrobial peptide LL-37, was more resistant to proteolytic degradation of human neutrophil elastase, S. aureus aureolysin, and V8 protease due to Trp-stabilized amphiphilic helix conformation and the high affinity of Trp to bacterial membrane bilayer [90]. In contrast, AvBD2-K31A derived from AvBD-2 by replacing the positively charged residue Lys31 with a neutral and hydrophobic residue Ala, showed significantly reduced antimicrobial activity compared to the wild-type AvBD-2 [21].

Modification of AMPs by introducing unnatural amino acids has been used to reduce salt sensitivity, a major drawback in the development of AMPs as therapeutic agents. It has been shown that replacing histidine (His) residues with $\beta$-naphthylalanine and $\beta-(4,4$ 'biphenyl) alanine improved both antimicrobial activity and salt resistance of histidine-rich peptide P-113, an active fragment of human histatin 5 secreted into the saliva [89]. Because host proteases cannot degrade peptides containing D-amino acid residues, incorporating Damino acids is another way to improve stability and reduce hemolytic activity of AMPs [92].

\subsubsection{Post-translational modification of AMPs}

Host AMPs are directly synthesized as mature active forms. Some post-translational modifications are necessary for improving their biological functions and stability and minimizing the cytotoxicity to host cells [12], including phosphorylation, methylation, amidation, glycosylation, and formation of the disulfide bonds. The post-translational 
modifications can also be applied to chemical synthesis of AMPs. For instance, synthetic antimicrobial peptide P60.4 Ac, the N-terminal acetylated and C-terminal amidated variant of P60.4, a fragment of human LL-37, showed higher antimicrobial activity than LL-37 against E. coli ATCC 8739, P. aeruginosa ATCC 9027, and C. albicans ATCC 10231 [91]. Therefore, posttranslational modifications should be considered in the design of novel AMPs.

\subsubsection{Other modifications}

Additional strategies have been utilized to design or modify AMPs, such as combining two different natural AMPs to enhance their salt-resistance and antimicrobial activity [93], designing AMPs with an $\alpha$-helical structure [94], and using computer-aided quantitative structure-activity relationship (QSAR) models to assess the relationship of measurable properties (such as charge and molecular weight) of AMPs [95]. For example, hybrid cationic AMP consisting of hBD-1 and $\theta$-defensin retained the high antimicrobial activity of hBD-1 with improved salt-resistance [93].

\section{Summary}

Antimicrobial peptides (AMPs), an evolutionarily conserved component of the innate immune system, are found in a wide range of hosts, including vertebrates, invertebrates, and plants. AMPs are broad-spectrum antimicrobial agents against bacteria, fungi, viruses, and protozoa. Their antimicrobial function is accomplished by targeting microbial membrane and intracellular components, which makes it difficult for bacteria to develop resistance. Several challenges must be overcome in order to develop AMPs as therapeutic and preventative agents, such as the sensitivity to cationic salts and proteases and cost of production. To maximize the favorable biological functions and minimize the undesirable 
properties, it is important to take the following factors into consideration: the amphipacity, net positive charge and charge distribution, hydrophobicity, length, and secondary structure, which collectively determine the antimicrobial activity, immune modulating activity, resistance to salts and proteases, cytotoxicity, and cost of production.

The main work of this dissertation focuses on the antimicrobial activity and structurefunction relationship of two AvBDs (AvBD-6 and AvBD-12). A series of short AMPs were designed by extracting key functional domains of AvBDs and further modifications to maximize their antimicrobial activity and resistance to salts and proteases. Data from in vitro and in vivo experiments demonstrated that two newly designed AMPs have great potentials as therapeutic and preventative agents. 


\title{
CHAPTER 2: Biological Functions of Avian $\beta$-Defensins (AvBDs):
}

\section{AvBD-6 and AvBD-12}

Published in BMC Microbiology (2016) 16:210.

\begin{abstract}
Avian beta-defensins (AvBD) are small, cationic, antimicrobial peptides. However, the mechanisms of action remain to be fully understood. The present study characterized the structure-function relationship of AvBD-6 and AvBD-12, two peptides with different net positive charges, same hydrophobicity and distinct tissue expression profiles. AvBD-6 was more potent than AvBD-12 against E. coli, S. Typhimurium, and $S$. aureus as well as clinical isolates of extended-spectrum beta-lactamase (ESBL)-producing E. coli and $K$. pneumoniae. AvBD-6 was more effective than AvBD-12 in neutralizing LPS and binding bacterial genomic DNA. Increasing bacterial concentration from $10^{5} \mathrm{CFU} / \mathrm{ml}$ to $10^{9}$ CFU/ml abolished AvBDs' antimicrobial activity. Increasing $\mathrm{NaCl}$ concentration significantly inhibited AvBDs' antimicrobial activity, but not the LPS-neutralizing function. Both AvBDs were mildly chemotactic for chicken macrophages and strongly chemotactic for $\mathrm{CHO}-\mathrm{K} 1$ cells expressing the chicken chemokine receptor 2 (CCR2).
\end{abstract} AvBD-12 at higher concentrations also induced migration of murine immature dendritic cells (DCs). Disruption of disulfide bonds abolished AvBDs' chemotactic activity. Neither AvBDs was toxic to $\mathrm{CHO}-\mathrm{K} 1$, macrophages, or DCs. AvBDs are potent antimicrobial peptides under low-salt conditions, effective LPS-neutralizing agents, and broad-spectrum chemoattractant peptides. In conclusion, their antimicrobial activity is positively correlated with AvBDs' net positive charges, inversely correlated with $\mathrm{NaCl}$ concentration and bacterial concentration, and minimally dependent on intramolecular disulfide bonds. In 
contrast, their chemotactic property requires the presence of intramolecular disulfide bonds. Above results provide a theoretical basis for developing AvBD-based therapeutic and immunomodulatory agents.

\section{Background}

Defensins are small cationic antimicrobial peptides containing six disulfide-paired cysteines [96]. Based on the connectivity of six conserved cysteine residues, defensins are classified into three subfamilies: $\alpha-, \beta$-, and $\theta$-defensins $[11,38,97]$. Due to the interest in their potential application as antibiotic alternatives, host defensins have been extensively investigated $[9,21,98,99]$. Although many defensins show broad-spectrum antimicrobial activities against bacteria, fungi, and some enveloped viruses, the mechanisms of action remain to be fully understood $[4,9,45,55]$. The antimicrobial mechanism of defensins primarily depends on several structural features, such as cationic charge and hydrophobicity, and is mainly divided into two classes, membrane-disruption and intracellular target $[21,32]$. The membrane-disruptive model has been attributed to the electrostatic attraction between positively charged amino acid residues and negatively charged microbial membrane components (such as lipopolysaccharides (LPS), lipoteichoic acid (LTA) and anionic phospholipids) and insertion of hydrophobic residues into the microbial membrane, resulting in membrane disruption and cell death $[45,100]$. Several models have been proposed to support this mechanism, including the barrel-stave, toroidal and carpet membrane pore-forming models, and sinking raft transient pore forming model $[8,11,16,87,101,102]$. In addition to the direct action on the microbial membrane, several studies have revealed intracellular functions of translocated antimicrobial peptides, such as 
interfering with cytoplasmic membrane septum formation and cell-wall integrity, binding to nucleic acids, and inhibiting enzyme function $[8,11,55]$.

Defensins also contribute to the adaptive immunity by chemoattracting monocytes, $\mathrm{T}$ lymphocytes, dendritic cells (DC) and mast cells to the site of infection, enhancing macrophage phagocytosis, inducing pro-inflammatory cytokines, and activating immature DCs $[45,55,103]$. The chemotactic activities of human $\beta$-defensin 1 to 3 (hBD1-3), as well as mouse $\beta$-defensin 2 (mBD2), are mediated by chemokine receptor CCR2 and CCR6 $[64,104-106]$. Activation of immature DCs by murine $\beta$-defensin- 2 and avian $\beta$-defensin13 involves the Toll-like receptor 4-nuclear factor-kappa B (TLR4-NFkB) signaling cascade $[107,108]$. These activities enable $\beta$-defensins function as indigenous vaccine adjuvants [109, 110].

The chicken genome contains $14 \beta$-defensin genes, located in a single defensin gene cluster on chromosome $3 q 3.5-3.7[40,111]$. Some of these defensin peptides were initially referred to as Gallinacins (Gal). To be consistent with the mammalian defensin nomenclature, the term of avian $\beta$-defensin (AvBD) was adopted [111-113]. The first two avian $\beta$-defensins, chicken AvBD-1 and AvBD-2, were isolated from chicken leukocytes in 1994, and later an inducible epithelially expressed avian $\beta$-defensin (AvBD-3) was reported in 2001 [44, 114, 115]. Transcriptional analysis of AvBD genes indicates that AvBD-1, -2 , and -4 to -7 are mainly expressed in myeloid, whereas the remaining AvBD3 , and -8 to -14 are strongly expressed in epithelial cells. Both myeloid and epithelial AvBDs are also expressed in a variety of other tissues [40, 111, 115]. Although many AvBDs possess certain degrees of antimicrobial activity and some may interact with immune cells, limited information is available regarding the mechanisms of antimicrobial 
and immunomodulatory activities $[9,55,116]$. In the present study, we have characterized the contribution of charge and disulfide bonds to various biological functions of AvBD-6 and AvBD-12. These two AvBDs are conserved in all birds and have the same hydrophobicity in their amino acid residues, whereas AvBD-12 has the lowest average net positive charge and AvBD-6 has the comparative higher average net positive charge in different birds according to the comparison of net charges of AvBDs [53].

\section{Methods and Materials}

\section{Peptides Synthesis}

The mature peptides of AvBD-6 (SPIHACRYQRGVCIPGPCRWPYYRVGSCGSGLKS CCVRNRWA [GenBank: AAT45546.1]) and AvBD-12 (GPDSCNHDRGLCRVGNCNP GEYLAKYCFEPVILCCKPLSPTPTK [GenBank: AAT48936.1]) were custom synthesized using the standard solid phase 9-fluorenylmethoxycarbonyl (Fmoc) method by LifeTein LLC (Hillsborough, NJ). Linear peptides were subjected to oxidative folding to ensure the correct formation of the three disulfide bonds between $\mathrm{Cys}^{1}-\mathrm{Cys}^{5}, \mathrm{Cys}^{2}-\mathrm{Cys}{ }^{4}$, and $\mathrm{Cys}^{3}-\mathrm{Cys}^{6}$. Following confirmation by mass spectrometry, AvBDs were purified by reversed-phase high-performance liquid chromatography (HPLC) and lyophilized (Lifetein, Hillsborough, NJ). The purity of the synthetic mature peptides was $>98 \%$, the molecular weight of AvBD-6 and AvBD-12 were 4738.57 and 4892.76, and the net charge of AvBD-6 and AvBD-12 at $\mathrm{pH} 7.0$ were +7 and +1 , respectively, and they have same hydrophobicity (Table 2-1).

\section{Bacteria}

Escherichia coli (E. coli, ATCC 25922), Salmonella enteric serovar Typhimurium (S. Typhimurium, ATCC 14028), and Staphylococcus aureus (S. aureus, ATCC 29213) were 
used to assess AvBDs' antimicrobial activity. Extended-spectrum beta-lactamase (ESBL) producing clinical isolates of E. coli and Klebsiella pneumoniae (K. pneumoniae) resistance to most beta-lactam antibiotics, including penicillin, cephalosporin, and monobactam, and methicillin-resistant S. pseudinetrmedius (Table 2-2) were included in the antimicrobial assays. All bacterial strains were grown and maintained on Trypticase Soy Agar (TSA) with 5\% Sheep Blood (Thermo Fisher Scientific) at $37^{\circ} \mathrm{C}$.

\section{Antimicrobial activity assay}

The bactericidal activity of AvBD-6 and AvBD-12 was determined by colony counting assay [57]. Three to five bacterial colonies from an overnight culture on a TSA agar plate were suspended in $5 \mathrm{ml}$ sterile distilled water to achieve a MacFarland standard of 0.5 (

$1.0 \times 10^{8}$ colony forming units per milliliter, $\left.\mathrm{CFU} / \mathrm{ml}\right)$. Ten microliters of bacterial

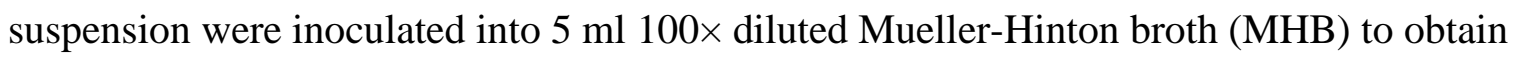
a final concentration of approximately $2 \times 10^{5} \mathrm{CFU} / \mathrm{ml}$. Twenty-five microliters of bacterial culture were mixed with $25 \mu$ of 2 -fold serially diluted AvBD- 6 and AvBD-12 in a Nunc ${ }^{\mathrm{TM}}$ 96-well polypropylene microtiter plate (Thermo Fisher Scientific), and the final concentrations of AvBDs were 1, 2, 4, 8, 16, 32, 64, $128 \mu \mathrm{g} / \mathrm{ml}$. Gentamycin $(100 \mu \mathrm{g} / \mathrm{ml})$ and peptide dilution buffer were used as positive and negative controls, respectively. Following an incubation at $37^{\circ} \mathrm{C}$ for $3 \mathrm{~h}, 10$-fold serial dilutions of the bacterial-peptide mixture were inoculated onto LB agar plates, then colonies were counted after $16 \mathrm{~h}$ of incubation at $37^{\circ} \mathrm{C}$. The bactericidal activity was expressed as percent of killing using the following formula: $\left(\mathrm{CFU}_{\text {control }}-\mathrm{CFU}_{\text {treated }}\right) / \mathrm{CFU}_{\text {control }} \times 100 \%$. To investigate the effect of ionic strength on the antimicrobial activity of AvBD-6 and AvBD-12, $5 \mathrm{mM}, 50 \mathrm{mM}$, and $150 \mathrm{mM} \mathrm{NaCl}$ were included in the incubation buffer with peptides at antimicrobial assay. 
MICs of AvBD-6 and AvBD-12 were determined using broth microdilution method according to the guidelines of Clinical and Laboratory Standards Institute (CLSI) [117, 118]. Minimum inhibitory concentrations were also determined using a low salt Muller Hinton broth and the values were presented as MIC-ls. In brief, AvBDs were prepared in serial two-fold dilutions in a 96-well polypropylene microtiter plate and inoculated with the bacteria at a final concentration of $5 \times 10^{5} \mathrm{CFU} / \mathrm{ml}$. The final concentrations of AvBDs ranged from 2 to $256 \mu \mathrm{g} / \mathrm{ml}$. After incubation at $37{ }^{\circ} \mathrm{C}$ for 16 to $24 \mathrm{~h}$, the lowest concentration that completely prevented visible bacteria growth was recorded. All antimicrobial assays were conducted in duplicate.

\section{Killing kinetics measurement}

The killing kinetics of AvBD-6 and AvBD-12 were confirmed against E. coli ATCC 25922, S. Typhimurium ATCC 14028 and S. aureus ATCC 29213. Equal volumes (50 $\mu 1)$ of bacterial suspension at the concentrations $2 \times 10^{5}, 2 \times 10^{7}$, and $2 \times 10^{9} \mathrm{CFU} / \mathrm{ml}$ and AvBDs at a final concentration of $32 \mu \mathrm{g} / \mathrm{ml}$ were co-incubated in a 96-well polypropylene microtiter plate at $37{ }^{\circ} \mathrm{C}$ for $30,60,90,120,150,180 \mathrm{~min}$. Bacterial-peptide mixtures were serially

diluted and plated on LB agar plates and colonies were counted after incubation at $37^{\circ} \mathrm{C}$ for $16 \mathrm{~h}$. The assays were repeated three times with duplicate samples.

\section{Limulus amoebocyte lysate assay}

The LPS-neutralizing abilities of AvBDs were assessed using a Limulus Amoebocyte Lysate (LAL) Chromogenic Endotoxin Quantitation Kit, (Pierce ${ }^{\mathrm{TM}}$, Thermo Fisher Scientific) according to the manufacturer's instructions. Briefly, equal volumes (25 $\mu \mathrm{l})$ of AvBDs in endotoxin-free water (final concentrations of 8, 16, $32 \mu \mathrm{g}$ ) and E. coli O111: B4

LPS or $S$. Typhimurium L6143 LPS (2 EU/ml) were co-incubated at $37{ }^{\circ} \mathrm{C}$ for $30 \mathrm{~min}$ to 
permit binding of the peptide to LPS. Then, $50 \mu 1$ of the assay mixture was transferred to Greiner CELLSTAR ${ }^{\circledR}$ flat-bottom, non-pyrogenic 96-well cell culture plate (VWR, Sugar Land, TX). Fifty microliters of 2-fold serially diluted E. coli O111: B4 LPS, ranging from 0.125 to $1.0 \mathrm{EU} / \mathrm{ml}$, were used as the standards. Endotoxin-free water and AvBDs alone were included as negative controls. Fifty microliters of LAL was added to each well of the assay plate which was incubated at $37{ }^{\circ} \mathrm{C}$ for $10 \mathrm{~min}$. One hundred $\mu \mathrm{l}$ of chromogenic substrate was then added to each well followed by incubation at $37^{\circ} \mathrm{C}$ for $6 \mathrm{~min}$, and the addition of $50 \mu \mathrm{l}$ of $25 \%$ acetic acid to stop the reaction. Color change due to the enzymatic liberation of $p$-Nitroaniline was monitored at $405 \mathrm{~nm}$ with Bio-Rad Benchmark Microplate Reader (Hercules, CA). LPS neutralizing rate was calculated as (OD405 AvBDs+LPS $\left.\mathrm{OD} 405_{\mathrm{H} 2 \mathrm{O}}\right) /\left(\mathrm{OD} 405_{\mathrm{LPS}}-\mathrm{OD} 405_{\mathrm{H} 2 \mathrm{O}}\right) \times 100 \%$. The assays were performed three times with duplicate samples.

\section{Cell culture}

Chicken macrophage MQ-NCSU and HD11 cell lines and Chinese hamster ovary (CHO)K1 cell line were maintained in RPMI-1640 media supplemented with $10 \%$ fetal bovine serum (FBS), $100 \mathrm{U} / \mathrm{ml}$ penicillin and $100 \mu \mathrm{g} / \mathrm{ml}$ streptomycin, at $37^{\circ} \mathrm{C}$ in humidified air with $5 \% \mathrm{CO}_{2}$. Additional $2 \%$ chicken serum was added to culture MQ-NCSU cells. Mouse immature dendritic cell line JAWSII (ATCC CRL-11904 ${ }^{\mathrm{TM}}$ ) was cultured in Alpha minimum essential medium with ribonucleosides, deoxyribonucleosides, $4 \mathrm{mM}$ Lglutamine, $1 \mathrm{mM}$ sodium pyruvate and $5 \mathrm{ng} / \mathrm{ml}$ murine Granulocyte macrophage-colony stimulating factor (GM-CSF), supplemented with 20\% FBS, $100 \mathrm{U} / \mathrm{ml}$ penicillin and 100 $\mu \mathrm{g} / \mathrm{ml}$ streptomycin, at $37^{\circ} \mathrm{C}$ in humidified air with $5 \% \mathrm{CO}_{2}$.

\section{Cell cytotoxicity}


The cytotoxicity of AvBDs to four cell lines was determined using MTT (3-(4, 5dimethylthiazol-2-yl)-2, 5-diphenyltetrazolium bromide, Thermo Fisher Scientific) cell proliferation assay. Cells $\left(5 \times 10^{3}\right.$ cells/well $)$ in 96 -well microtiter tissue culture plates were treated with AvBD-6 and AvBD-12 at the concentrations of 4, 16, 64, and $256 \mu \mathrm{g} / \mathrm{ml}$ for $4,12,24,48 \mathrm{~h}$ at $37^{\circ} \mathrm{C}$. After treatment, $20 \mu \mathrm{l}$ of $12 \mathrm{mM}$ MTT solution was added to each well and the plates were incubated for $4 \mathrm{~h}$. The medium in each well was replaced with dimethyl sulfoxide (DMSO, Sigma-Aldrich) to dissolve MTT crystals. The plates were read using a spectrophotometer at $540 \mathrm{~nm}$. The viability of treated cells was expressed as the percentage of viability relative to the untreated control. The experiment was performed in triplicate.

\section{Construction of pAcGFP1-N1-CCR2 and -CCR6}

Total RNA was extracted from MQ-NCSU cells or chicken liver with the Rneay ${ }^{\circledR}$ Mini kit (Qiagen), and the first-strand cDNA was synthesized by reverse-transcription polymerase chain reaction (RT-PCR) with SuperScript ${ }^{\circledR}$ III first-strand synthesis system (Invitrogen) according to the manufacturer's instruction. PCR was performed using Pfu DNA polymerase (Stratagene, CA) with primers containing flanking enzyme restriction sites XhoI and HindIII. The forward and reverse primers of CCR2 and CCR6 were as follows: CCR2-F 5'-CCGCTCGAGGCCACCATGGAGAACTATACTGACT-3’, CCR2-R 5’-CC CAAGCTTCAGTCCAGTAGAGATGTC-3’; CCR6-F 5’-CCGCTCGAGGCCACCATG AGTACTACAGTTTTTG-3’ and CCR6-R 5'-CCCAAGCTTTATAGTAAAAGAAGAT GCAT-3'. The PCR products of CCR2 and CCR6 were purified and cloned to $\mathrm{pCR}^{\circledR} 2.1$ $\mathrm{TOPO}^{\circledR}$ vector and transformed into $E$. coli $\mathrm{TOP}^{\circledR} \mathrm{F}^{\prime}$ competent cells. Plasmid DNA was isolated from the transformed clones using the QIAprep ${ }^{\circledR}$ Spin Miniprep kit (Qiagen) and 
sequenced to confirm the identity of inserts. The CCR2 and CCR6 inserts were subcloned to eukaryotic expression vector pAcGFP1-N1 (Clontech) between the XhoI and HindIII restriction sites and transformed into $E$. coli $\mathrm{TOP}_{10 \mathrm{~F}^{\prime}}$ competent cells. The recombinant pAcGFP1-N1-CCR2 and -CCR6 plasmids were extracted and confirmed by PCR and XhoI and HindIII digestion.

\section{Transfection of CHO-K1 cells expressing CCR-2 and CCR-6}

CHO-K1 cells were seeded into a 6-well-plate. After $80 \%$ confluence, cells were transfected with pAcGFP1-N1-CCR2, pAcGFP1-N1-CCR6 and pAcGFP1-N1 (mock) plasmids using the TransIT-CHO Transfection Kit according to the manufacturer's instructions (Mirus Bio LLC, WI). After $72 \mathrm{~h}$ incubation, cells were harvested and cultured in selective media in supplement of $500 \mu \mathrm{g} / \mathrm{ml}$ G418 (Sigma-Aldrich). CHO-K1 cells stably expressing CCR2 and CCR6 were separated by flow cytometry and used to perform chemotaxis assay.

To verify transfection, CCRs transfected $\mathrm{CHO}-\mathrm{K} 1$ cells were cultured on a cover slide in RPMI-1640 with 10\% FBS, 2mM L-glutamine, 100 units/ml penicillin and streptomycin, $500 \mu \mathrm{g} / \mathrm{ml} \mathrm{G8}$ at $37{ }^{\circ} \mathrm{C}$ in humidified air with $5 \% \mathrm{CO}_{2}$. After $80 \%$ confluence, cells were washed with ice-cold phosphate buffered saline (PBS) and fixed by methanol: acetone (v: $\mathrm{v}, 1: 1)$. Fixed cells were then washed twice with PBS and stained nuclei with $0.1 \mu \mathrm{g} / \mathrm{ml}$ DAPI (Thermo Fisher Scientific) in PBS for 1 min, then the cells were washed three times with PBS before microscopic visualization. Wild-type CHO-K1 cells and mock-transfected cells were examined simultaneously as controls. Cells were visualized and captured using Nikon fluorescent microscope connected with Olympus DP2-BSE software (Eclipse E600, Japan, 20x). In addition, total RNAs were extracted from transfected cells and RT-PCRs 
were performed to verify expression of CCR2 and CCR6 as described in the construction of pAcGFP1-N1-CCR2 and-CCR6. PCR products were subjected to gel electrophoresis and photographed on the FluorChem Q imaging system (Cell Biosciences, CA).

Western blot analysis was also carried out to confirm CCR2/CCR6 expression in CHO-K1 cells. In brief, CHO-K1 cells were suspended in RIPA cell lysis buffer (Thermo Fisher Scientific) for $10 \mathrm{~min}$ and centrifuged at $13,000 \mathrm{~g}$ for $10 \mathrm{~min}$ at $4{ }^{\circ} \mathrm{C}$. Protein concentration was measured using a NanoDrop 1000 spectrophotometer at $280 \mathrm{~nm}$ (Thermo Fisher Scientific). An equivalent amount of protein from each sample was run on a $12 \%$ SDSPAGE gel and transferred onto a nitrocellulose membrane (Bio-Rad). The protein fractions were probed with a polyclonal goat anti-GFP primary antibody (1:1000, Thermo Fisher Scientific) and horseradish peroxidase (HRP)-coupled anti-goat secondary antibody (1:500, Promega), and visualized after color development with 4-Chloro-1-Naphthol (4-CN) and $30 \%$ hydrogen peroxide (Thermo Fisher Scientific). Protein bands were photographed using FluorChem Q imaging system (Cell Biosciences, CA).

\section{Chemotaxis assay}

Migration of MQ-NCSU cells, JAWSII cells and CCR2-/CCR6-expressing CHO-K1 cells in response to chemotactic factors were determined using a 48-well microchemotaxis chamber technique as previously described [119]. The single cell suspension was prepared by treating cells with a non-enzymatic cell dissociation solution (Sigma-Aldrich), and the dissociated cells were harvested by centrifugation and resuspended in chemotaxis assay buffers at a final concentration of $1.5 \times 10^{6}$ cells $/ \mathrm{ml}$. The chemotaxis buffer for MQ-NCSU and CCR2-/CCR6-expressing CHO-K1 cells was RPMI 1640 supplement with 0.1\% BSA, 100U/ml penicillin, and $100 \mu \mathrm{g} / \mathrm{ml}$ streptomycin, and for JAWSII cells was Alpha 
Minimum Essential Medium containing 0.1\% BSA, 100U/ml penicillin, and $100 \mu \mathrm{g} / \mathrm{ml}$ streptomycin. In brief, $28 \mu 1$ of 4-fold diluted AvBDs in chemotactic buffer ranging from 0.25 to $64 \mu \mathrm{g} / \mathrm{ml}$ was placed in the lower wells of a 48 -well microchemotaxis chamber (Neuro Probe, San Diego, CA), and $50 \mu 1$ of cell suspension was added to the upper wells. The lower and upper compartments were separated by a pre-coated polycarbonate membrane ( $8 \mu \mathrm{m}$ pore size) with $10 \mu \mathrm{g} / \mathrm{ml}$ fibronectin (BD Biosciences, Bedford, MA). After incubation at $37^{\circ} \mathrm{C}$ for 1 to $5 \mathrm{~h}$ in humidified air with $5 \% \mathrm{CO}_{2}$, the membranes were removed, top side scraped, stained with Kwik-Diff staining kit (Thermo Fisher Scientific), and counted under light microscopy. Chemotaxis buffer and bacterial peptide $\mathrm{N}$-formylmethionyl-leucyl-phenylalanine (fMLF, Sigma-Aldrich) were used as negative and positive controls, respectively. The results were presented as chemotactic indexes (C.I.) using the following formula: C.I. $=$ the number of migrated cells following treatment with AvBDs / the number of migrated cells following treatment with chemotactic buffer. To investigate if the peptides directly bind to plasma membrane components, JAWSII cells were pre-incubated with $0.25-64 \mu \mathrm{g} / \mathrm{ml}$ chemoattractant (AvBD-6 or AvBD-12) for 30 min at $37^{\circ} \mathrm{C}$ in humidified air containing $5 \% \mathrm{CO}_{2}$. Then, the chemotaxis assay was performed with the same procedure.

For CCR2/6 expressing CHO-K1 cells, the mock-transfected CHO-K1 cells were analyzed simultaneously as negative cell control. Recombinant chicken CCL20 (Innovative research, the chemokine ligand for CCR6) was used as the positive chemotactic agent control. C.I. = the number of migrated CCR2/6-expressing CHO-K1 cells induced by AvBDs / the number of migrated cells induced by the chemotactic buffer. All chemotaxis assays were performed five times. 


\section{Reduction of AvBD peptides}

Ten microgram of AvBDs were incubated with $0.8 \mathrm{mM} \beta$-nicotinamide adenine dinucleotide 2'-phosphate, reduced (NADPH), $0.2 \mu \mathrm{M}$ thioredoxin reductase, $0,1,2,4 \mu \mathrm{M}$ E.coli thioredoxin (Sigma-Aldrich) at $37{ }^{\circ} \mathrm{C}$ for $60 \mathrm{~min}$, in $0.1 \mathrm{M}$ potassium phosphate2mM EDTA buffer with $\mathrm{pH}$ 7.0. The incubation mixtures were analyzed using reversedphase high-performance liquid chromatography (RP-HPLC) and the absorbance at $340 \mathrm{~nm}$ was monitored to test the consumption of NADPH using a NanoDrop 1000 spectrophotometer (Thermo Fisher Scientific). The reduced peptides were separated from thioredoxin system by Amicon Ultra centrifuge filters with $10 \mathrm{kDa}$ membrane and $3 \mathrm{kDa}$ membrane (Millipore).

Natural and reduced AvBDs were analyzed by RP-HPLC using the Hitachi HPLC system and Phenomenex (Torrance, CA) Luna ${ }^{\circledR}$ C18 (2) Columns $(100 \times 4.6 \mathrm{~mm}, 3 \mu \mathrm{m})$, mobile phase $90 \%$ A (water $+0.1 \%(\mathrm{v} / \mathrm{v})$ trifluoroacetic acid (TFA)) and 10\% B (acetonitrile + $0.1 \%(\mathrm{v} / \mathrm{v}) \mathrm{TFA})$, under $214 \mathrm{~nm} \mathrm{UV}$ wavelength, and a flow rate of $1.0 \mathrm{ml} / \mathrm{min}$ at $25{ }^{\circ} \mathrm{C}$. The structures of AvBDs were probed by circular dichroism (CD) spectroscopy on a JASCO J-815 spectropolarimeter (JASCO, Easton, MD) in a $0.2 \mathrm{~cm}$ quartz cell. Spectra were recorded at peptide concentrations of $0.1 \mathrm{mg} / \mathrm{ml}$ diluted in a $10 \mathrm{mM}$ phosphate buffer ( $\mathrm{pH}$ 7.4), in far-ultraviolet (UV) region from 190 to $250 \mathrm{~nm}$ at room temperature. Each CD spectrum was the average of six consecutive scans, and all data were corrected with the blank buffer and expressed as molar ellipticity $\theta\left(\mathrm{deg} \cdot \mathrm{cm}^{2} \cdot \mathrm{mol}^{-1}\right)$.

\section{Transmission electron microscopy (TEM)}

Mid-logarithmic phase $S$. Typhimurium cells $\left(1 \times 10^{8} \mathrm{CFU} / \mathrm{ml}\right)$ were treated with wild-type and reduced AvBD-6 (16 $\mu \mathrm{g} / \mathrm{ml})$ and AvBD-12 $(128 \mu \mathrm{g} / \mathrm{ml})$ at $37{ }^{\circ} \mathrm{C}$ for $30 \mathrm{~min}$ in the 
presence of $5 \mathrm{mM} \mathrm{NaCl}$. These AvBD concentrations were required to inhibit bacterial growth in the presence of $5 \mathrm{mM} \mathrm{NaCl}$. Bacterial cells were harvested by centrifugation at 5, $000 \mathrm{~g}$ for $10 \mathrm{~min}$. TEM imaging was performed according to the procedure described by Park and Kand [120]. In brief, samples were fixed in Karnovsky's reagent (2\% paraformaldehyde, $2.5 \%$ glutaraldehyde in $0.1 \mathrm{M}$ sodium cacodylate buffer, $\mathrm{pH}$ 7.4) and dehydrated through a graded ethanol series of $20,50,70,90 \%$ and $3 \times 100 \%$, and then stained with $2 \%$ uranyl acetate. Observations were made under a transmission electron microscope (JEOL 1400, Japan). Untreated cells were processed at the same time as a negative control.

\section{Gel retardation assay}

The binding of AvBDs to genomic DNA of $S$. Typhimurium was evaluated by gel retardation assay [22]. The genomic DNA of $S$. Typhimurium was extracted using Genomic DNA kit (Qiagen). AvBDs were mixed with $0.4 \mu \mathrm{g}$ of genomic DNA at peptideto-DNA ratios of $0,0.5,1,2,4$ and $8(\mathrm{w} / \mathrm{w})$. BSA was used as the negative control. The mixture was incubated for $10 \mathrm{~min}$ at room temperature and analyzed by electrophoresis on a $0.5 \%$ agarose gel. Densitometric analysis of photographed gel pictures was performed using ImageJ software (NIH, Bethesda, MD, USA. The data were expressed as the density ratio of migrated DNA post- binding by AvBD or BSA to DNA pre-binding. The assay was performed in triplicate.

\section{Statistical analysis}

Data were expressed as means \pm standard deviation (SD) and analyzed by Student's $t$-test or one-way analysis of variance (ANOVA) followed by Duncan's test for multiple comparisons using software SPSS version 19.0 (IBM Corp., Armonk, NY). Differences at 
$p<0.05$ level were considered statistically significant, and at $p<0.01$ level were considered extremely significant.

\section{Results}

\section{Antimicrobial activity of AvBD-6 and AvBD-12}

Both AvBDs showed dose-dependent (1 to $128 \mu \mathrm{g} / \mathrm{ml}$ ) bactericidal activities against three common bacterial pathogens, E. coli, S. Typhimurium, and S. aureus (Figure 2-1). To detect the antimicrobial activity of AvBDs at a wide range of concentrations, percentage killing of bacteria was calculated. AvBD-6 was generally more potent than AvBD-12 in killing E. coli (2 to $64 \mu \mathrm{g} / \mathrm{ml}$ ), $S$. Typhimurium and $S$. aureus (8 to $128 \mu \mathrm{g} / \mathrm{ml}$ ). The susceptibility of three bacterial pathogens to AvBDs (AvBD-6 at lower concentrations and AvBD-12 at higher concentrations) could be classified as follows: E. coli $>S$. Typhimurium $>$ S. aureus. The killing activities of AvBDs were impaired by increasing $\mathrm{NaCl}$ concentration from $5 \mathrm{mM}$ to $50 \mathrm{mM}$ or $150 \mathrm{mM}$ (Figure 2-1). The negative impact of increased $\mathrm{NaCl}$ concentration could be summarized as follows: AvBD-6/AvBD-12/E. coli > AvBD-6/S. Typhimurium > AvBD-6/S. aureus > AvBD-12/S. Typhimurium > AvBD-12/S. aureus.

Since both AvBDs demonstrated significant killing activities at $32 \mu \mathrm{g} / \mathrm{ml}$, this concentration was used to characterize the killing kinetics. Log reduction of the bacterial colony-forming unit (CFU) was used to define the killing activity which enabled quantifying the number of bacteria killed when different concentrations of inoculum were tested. The killing kinetic study indicated that majority of the killing activity occurred within $30 \mathrm{~min}$ of bacteria-AvBD interaction (Figure 2-2). Killing activity decreased significantly when bacterial inoculum concentration increased from $10^{5} \mathrm{CFU} / \mathrm{ml}$ to $10^{9}$ 
$\mathrm{CFU} / \mathrm{ml}(p<0.01)$. A comparison of the killing activities of both AvBDs confirmed that AvBD-6 was more potent than AvBD-12.

The minimum inhibitory concentrations (MICs) of both AvBDs against reference ATCC strains and clinical isolates were above $128 \mu \mathrm{g} / \mathrm{ml}$ (Table 2-2). When a low salt MullerHinton broth was used to evaluate the minimum inhibitory concentrations (MIC-ls), much lower concentrations were required to inhibit the growth of extended-spectrum betalactamase (ESBL)-positive E. coli (AvBD-6 $\leq 8 \mu \mathrm{g} / \mathrm{ml}$ and AvBD-12 $\leq 64 \mu \mathrm{g} / \mathrm{ml}$ ) and $K$. pneumoniae (AvBD-6 = $16 \mu \mathrm{g} / \mathrm{ml}$ and AvBD-12 $\leq 64 \mu \mathrm{g} / \mathrm{ml})$. 
Table 2-1. Amino acid sequence and properties of AvBD-6 and AvBD-12

\begin{tabular}{|c|c|c|c|c|c|}
\hline Peptide & Amino acid sequence ${ }^{a}$ & $\begin{array}{c}\text { Length } \\
\text { (aa) }\end{array}$ & $\begin{array}{l}\text { Molecular } \\
\text { weight (Da) }\end{array}$ & Charge & $\begin{array}{c}\text { Hydropho } \\
\text { bicity }\end{array}$ \\
\hline AvBD-6 & $\begin{array}{l}\text { SPIHAC }{ }^{1} \text { RYQRGVC }{ }^{2} \text { IPGPC } \\
{ }^{3} \text { RWPYYRVGSC }{ }^{4} \text { GSGLKS } \\
C^{5} C^{6} \text { VRNRWA }\end{array}$ & 42 & 4738.57 & +7 & $33 \%$ \\
\hline AvBD-12 & $\begin{array}{l}\text { GPDSC }{ }^{1} \text { NHDRGLC }{ }^{2} \text { RVGN } \\
\mathrm{C}^{3} \text { NPGEYLAKYC }{ }^{4} \text { FEPVIL } \\
C^{5} C^{6} \text { KPLSPTPTKT }\end{array}$ & 45 & 4892.76 & +1 & $33 \%$ \\
\hline
\end{tabular}

${ }^{a}$ The three disulfide bonds formed between $\mathrm{Cys}^{1}-\mathrm{Cys}^{5}, \mathrm{Cys}^{2}-\mathrm{Cys}^{4}$ and $\mathrm{Cys}^{3}-\mathrm{Cys}{ }^{6}$.

Table 2-2. Minimum inhibitory concentrations of AvBD-6 and AvBD-12a

\begin{tabular}{|c|c|c|c|c|c|}
\hline \multirow{2}{*}{ Microorganism } & \multirow{2}{*}{$\begin{array}{c}\text { Source } \\
\text { (Number of strains) }\end{array}$} & \multicolumn{2}{|c|}{ AvBD-6 } & \multicolumn{2}{|c|}{ AvBD-12 } \\
\hline & & MIC & MIC-ls & MIC & MIC-Is \\
\hline \multicolumn{6}{|l|}{ Gram-negative } \\
\hline Escherichia coli & ATCC 25922 (1) & 128 & 4 & 256 & 32 \\
\hline Escherichia coli & Clinical isolate (10) & 256 & 8 & 256 & 64 \\
\hline $\begin{array}{l}\text { Salmonella enterica } \\
\text { serovar Typhimurium }\end{array}$ & ATCC 14028 (1) & $\geq 256$ & 16 & $>256$ & 128 \\
\hline Klebsilla pneumoniae & Clinical isolate (10) & $\geq 256$ & 16 & $>256$ & 64 \\
\hline \multicolumn{6}{|l|}{ Gram-positive } \\
\hline Staphylococcus aureus & ATCC 29213 (1) & 256 & 128 & $>256$ & $>256$ \\
\hline $\begin{array}{l}\text { Staphylococcus } \\
\text { pseudinetrmedius }\end{array}$ & Clinical isolate (10) & $\geq 256$ & $\geq 256$ & $>256$ & $>256$ \\
\hline
\end{tabular}

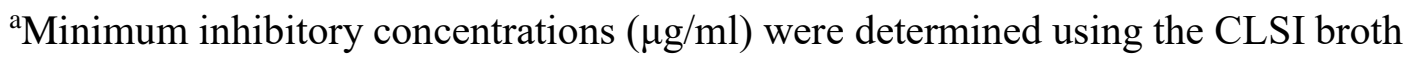
microdilution method and the modified broth microdilution method using low salt Muller Hinton Broth (MIC-ls) as described in materials and methods section. 


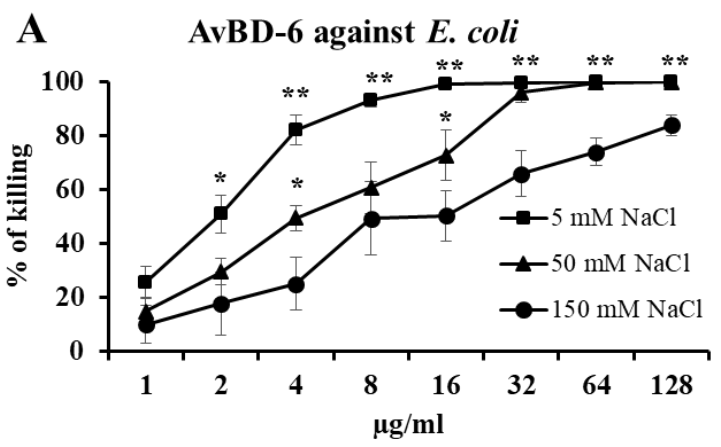

AvBD-6 against $S$. Typhimurium

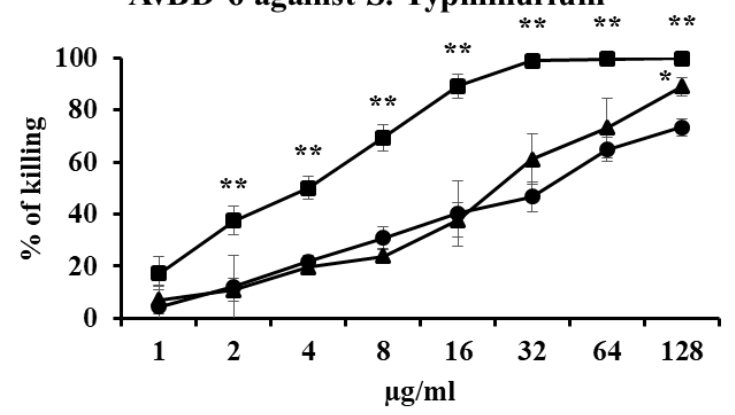

AvBD-6 against $S$. aureus * *

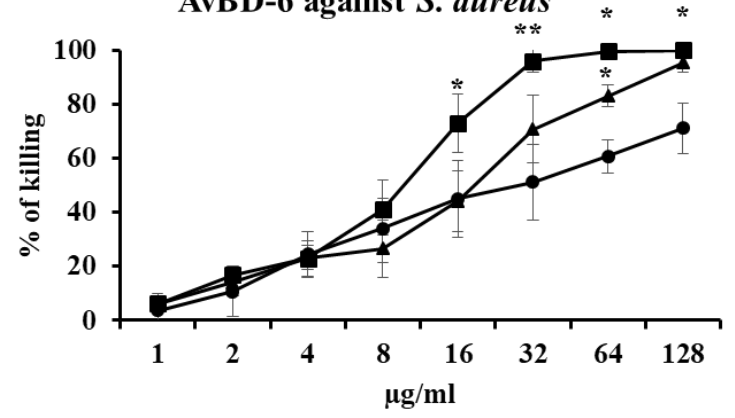

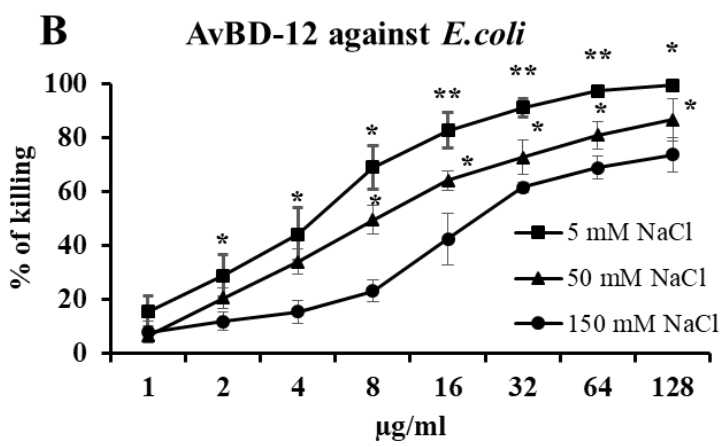

AvBD-12 against $S$. Typhimurium

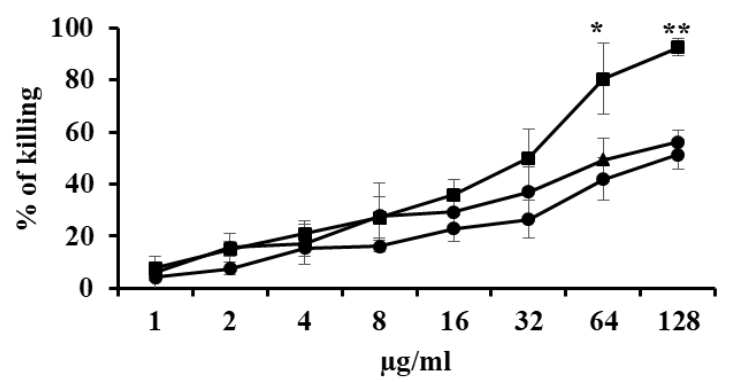

AvBD-12 against $S$. aureus

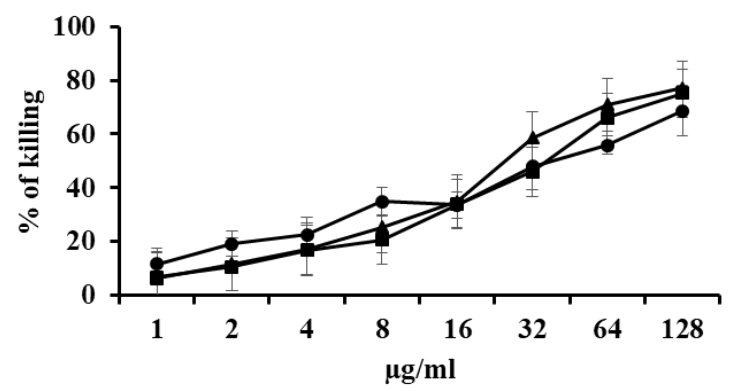

Figure 2-1. Antimicrobial activity of AvBD-6 and AvBD-12 against $E$. coli, $S$. Typhimurium and S. aureus

Bacteria $\left(10^{5} \mathrm{CFU} / \mathrm{ml}\right)$ were incubated with various concentrations of AvBD-6 or AvBD-

12 in the presence of $5 \mathrm{mM} \mathrm{NaCl}(\boldsymbol{\bullet}), 50 \mathrm{mM} \mathrm{NaCl}(\boldsymbol{\Delta})$, or $150 \mathrm{mM}(\bullet)$ at $37^{\circ} \mathrm{C}$ for $3 \mathrm{~h}$. (A) Antimicrobial activity of AvBD-6. (B) Antimicrobial activity of AvBD-12. Antimicrobial activity was presented as percent of killing as compared to no-AvBD control. Data are means of three independent experiments \pm SD $(n=3)$. Asterisks indicate statistically significant difference between $5 \mathrm{mM}$ and $50 \mathrm{mM}$ or $150 \mathrm{mM}$ of $\mathrm{NaCl}\left({ }^{*} p<\right.$ $0.05, * * p<0.01)$ 

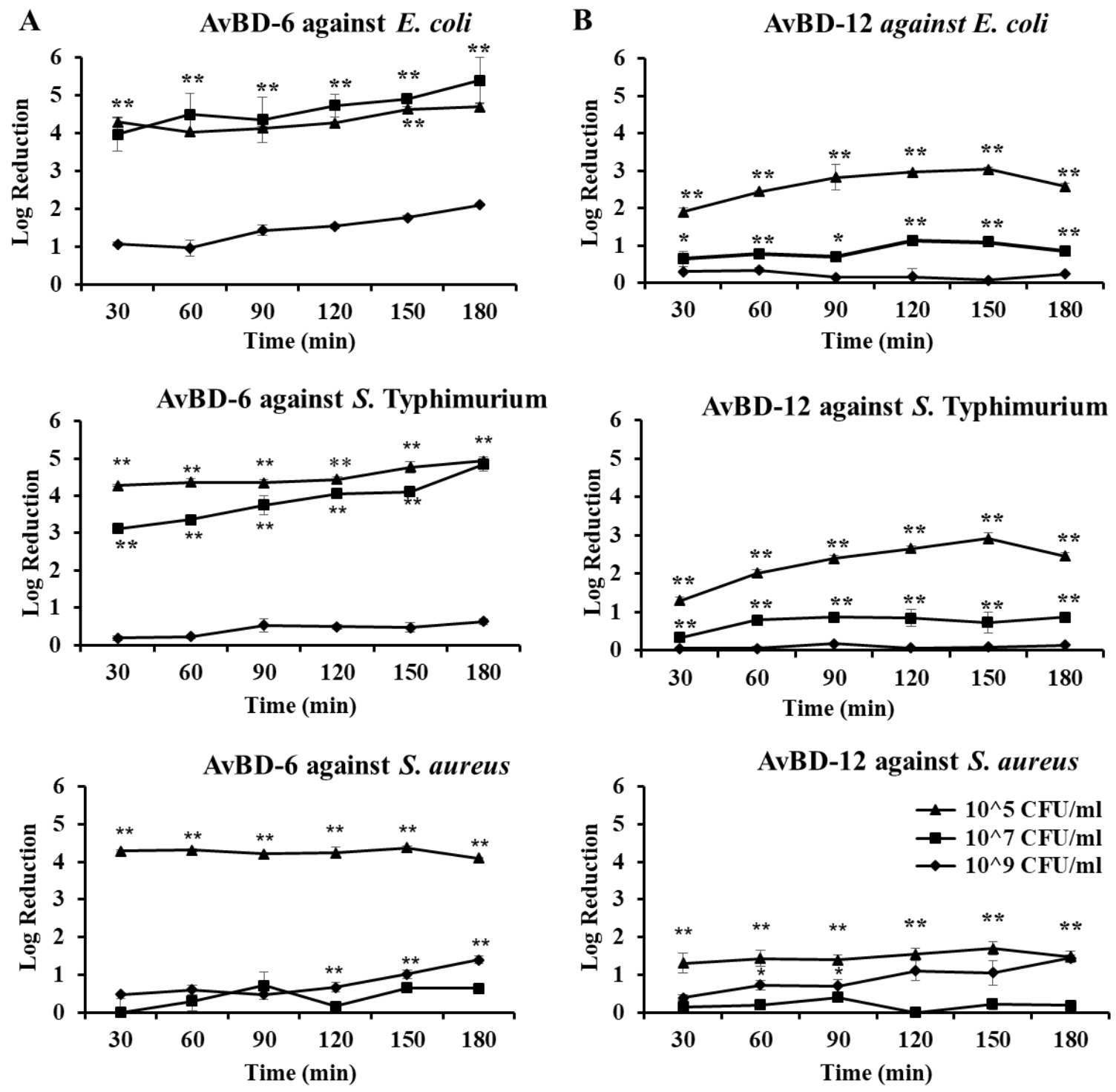

Figure 2-2. Killing kinetics of AvBD-6 and AvBD-12

Bacteria $\left(10^{5}, 10^{7}\right.$, and $\left.10^{9} \mathrm{CFU} / \mathrm{ml}\right)$ were treated with $32 \mu \mathrm{g} / \mathrm{ml}$ of at $37^{\circ} \mathrm{C}$ for different time points. (A) Killing kinetics of AvBD-6. (B) Killing kinetics of AvBD-12. Log reduction of $\mathrm{CFU}$ at each time point for each bacterial concentration was used to demonstrate the absolute number (relative to \% killing) of bacteria killed by AvBDs. Data are presented as means $\pm \mathrm{SD}(\mathrm{n}=3)$. Asterisks denote significant difference $\left({ }^{*} p<0.05,{ }^{* *} p\right.$ $<0.01)$ in killing activity of each AvBD between different bacterial concentrations: $10^{5}$ $\mathrm{CFU} / \mathrm{ml}(\boldsymbol{\Delta}), 10^{7} \mathrm{CFU} / \mathrm{ml}(\mathbf{\bullet})$, and $10^{9} \mathrm{CFU} / \mathrm{ml}(\bullet)$. 


\section{The ability of AvBD to neutralize LPS}

Both AvBD-6 and AvBD-12 neutralized LPS activity in a dose-dependent manner (Figure 2-3A). At the concentration of $32 \mu \mathrm{g} /$ well, AvBDs were able to neutralize more than $70 \%$ of the equal volume of $1 \mathrm{EU} / \mathrm{ml}$ of LPS. The neutralizing capacity of AvBD-6 was significantly stronger $(p<0.05$ or 0.01$)$ than that of AvBD-12. While AvBD-6 $(16 \mu \mathrm{g} / \mathrm{ml})$ was more effective in neutralizing $E$. coli LPS than $S$. Typhimurium LPS, AvBD-12 showed no difference in neutralizing $E$. coli LPS and S. Typhimurium LPS. Interestingly, $\mathrm{NaCl}$ concentrations, ranging from $0.1 \%(17.1 \mathrm{mM})$ to $0.8 \%(137 \mathrm{mM})$, had no impact on AvBDs' ability to neutralize LPS (Figure 2-3B).

\section{Cell Cytotoxicity}

The cytotoxicity of AvBD-6 and AvBD-12 to chicken macrophage cell line HD11 and MQ-NCSU, mouse immature dendritic JAWSII cells, and hamster CHO-K1 cells, was evaluated using an MTT cell proliferation assay (Thermo Fisher Scientific). Exposure of cells to AvBDs at concentrations of 4, 16, 64, $256 \mu \mathrm{g} / \mathrm{ml}$ for 4, 12, 24, and $48 \mathrm{~h}$ did not cause any change in cell viability. Data at the highest AvBD concentration $(256 \mu \mathrm{g} / \mathrm{ml})$ and longest exposure were presented in Figure 2-4. 

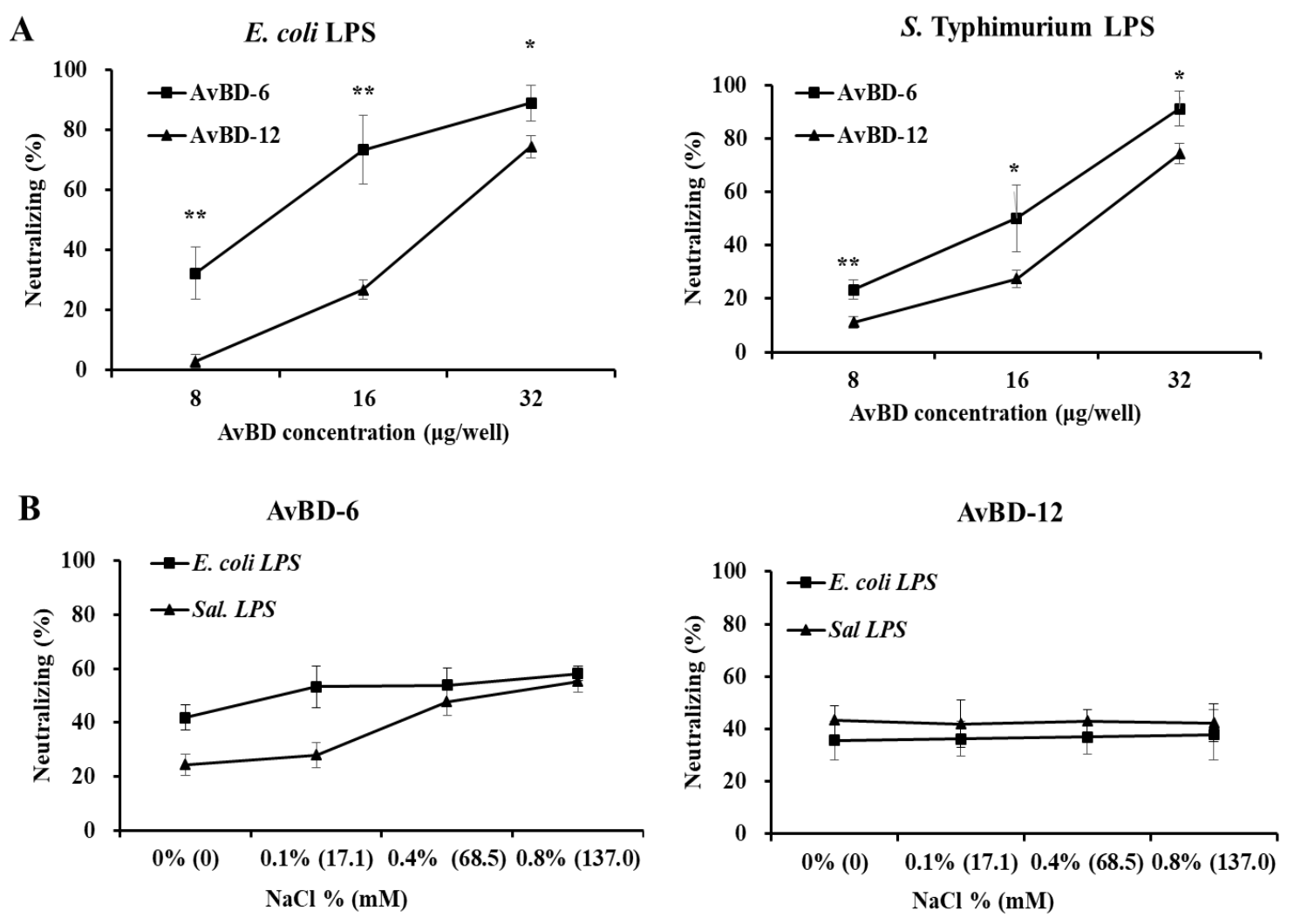

\section{Figure 2-3. The LPS-neutralizing activity of AvBD-6 and AvBD-12}

LPS-neutralizing activities of AvBD-6 and AvBD-12 were determined by the Limulus Amoebocyte Lysate (LAL) assay. (A) Neutralizing activities AvBD-6 (-) and AvBD-12 (ム) for E. coli O111: B4 LPS and S. Typhimurium L6143 LPS. (B) The effect of NaCl concentration on the ability of AvBDs to neutralize E. coli O111: B4 LPS (- $)$ and $S$. Typhimurium L6143 LPS ( $\mathbf{\Delta})$. The data are presented as means \pm SD $(n=3)$. Asterisks denote statistically significant difference in LPS-neutralizing activities between AvBD-6 and AvBD-12 $\left({ }^{*} p<0.05, * * p<0.01\right)$. 

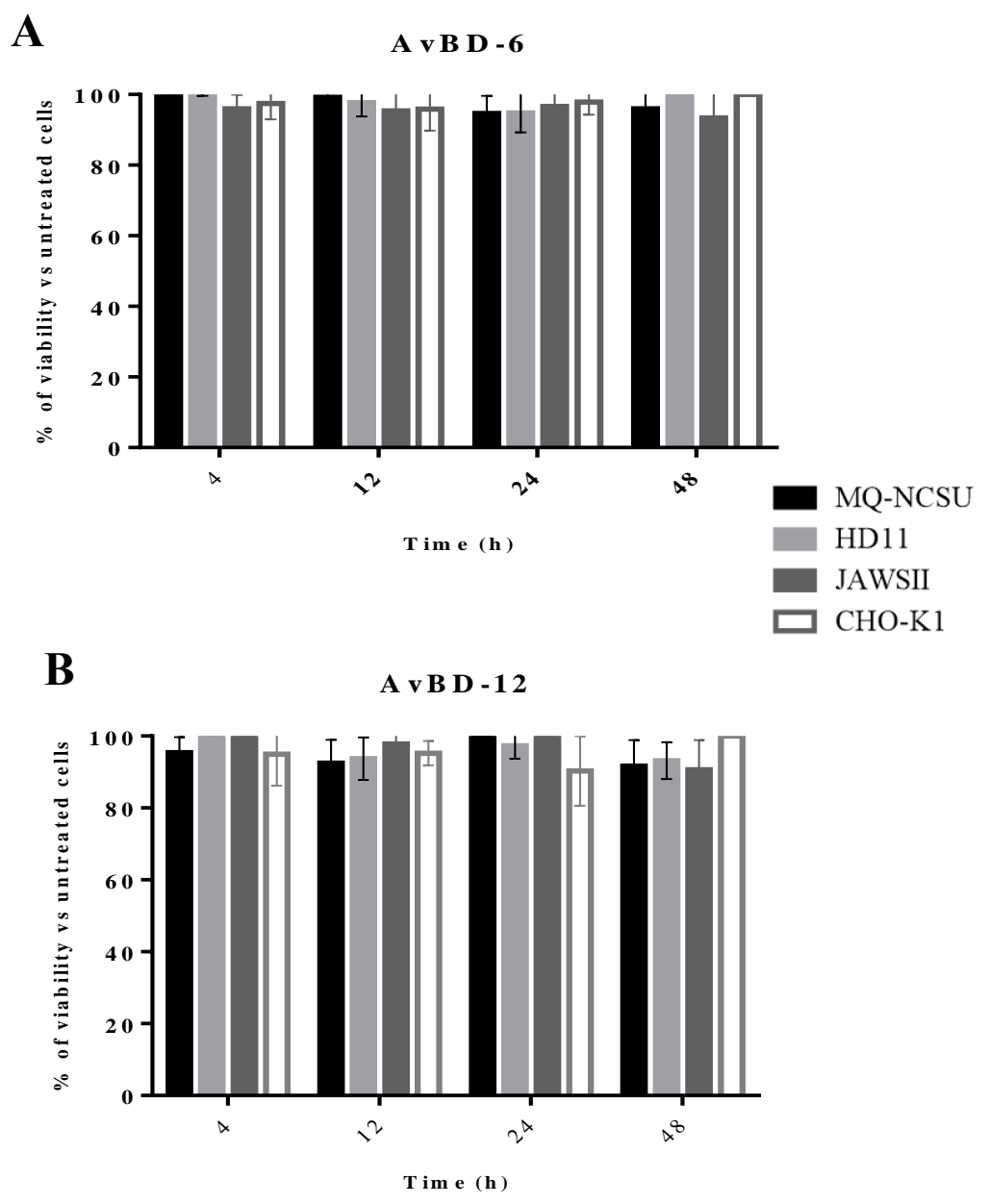

Figure 2-4. Cytotoxicity of AvBD-6 and AvBD-12 on host cells

Effect of $256 \mu \mathrm{g} / \mathrm{ml}$ AvBD-6 and AvBD-12 on the viability of chicken macrophages MQNCSU and HD11 cells, mouse immature dendritic JAWSII, and hamster CHO-K1 cells at $4,12,24,48 \mathrm{~h}$ of incubation. Results are shown as the percentages of viable cells in different treatment groups of AvBDs relative to the untreated control cells. The data are expressed as the mean \pm SD $(n=3)$. 


\section{Expression of CCR2-GFP/CCR6-GFP proteins in CHO-K1 cells}

Fluorescent microscopy showed that CCR2-GFP fusion protein (green fluorescence) was mainly expressed in the cytoplasmic membrane of transfected $\mathrm{CHO}-\mathrm{K} 1$ cells whereas CCR6-GFP was mostly expressed in the cytoplasm and GFP alone was visible throughout the cytoplasm and nucleus of CHO-K1 cells (Figure 2-5A). Wild-type CHO-K1 cells did not show any green fluorescence. The expression of CCR2 and CCR6 in transfected cells was confirmed by RT-PCR which amplified the CCR2 and CCR6 genes with the expected sizes (1,065 bp for CCR2 and 1,089 bp for CCR6, shown in Figure 2-5B). The expression of fusion proteins (CCR2-GFP, $65 \mathrm{kDa}$; CCR6-GFP, $66 \mathrm{kDa}$ ) was also confirmed by western blot analysis (Figure 2-5C).

\section{Chemotactic activity of AvBDs}

Both AvBDs demonstrated relatively low chemotactic activity for avian macrophage cell line MQ-NCSU (Figure 2-6A). At a high concentration (64 $\mu \mathrm{g} / \mathrm{ml})$, both AvBDs induced the migration of murine immature dendritic cells (JAWSII) and AvBD-12 was significantly more effective than AvBD-6 in chemoattracting JAWSII cells (Figure 2-6B). Pretreatment of JAWSII cells with AvBDs significantly inhibited subsequent cell migration towards AvBDs $(p<0.05$ ), confirming that migration of JAWSII was induced by AvBDs (Figure 2-6C and 6D). Both AvBDs exhibited a dose-dependent chemotactic effect on CCR2-CHO cells and no difference in their chemoattractant activities was detected (Figure 2-6E). AvBD-6 also showed a dose-dependent chemotactic activity for CCR6-CHO cells whereas AvBD-12 demonstrated minimal chemotactic activity for CCR6-CHO cells (Figure 2-6F). 


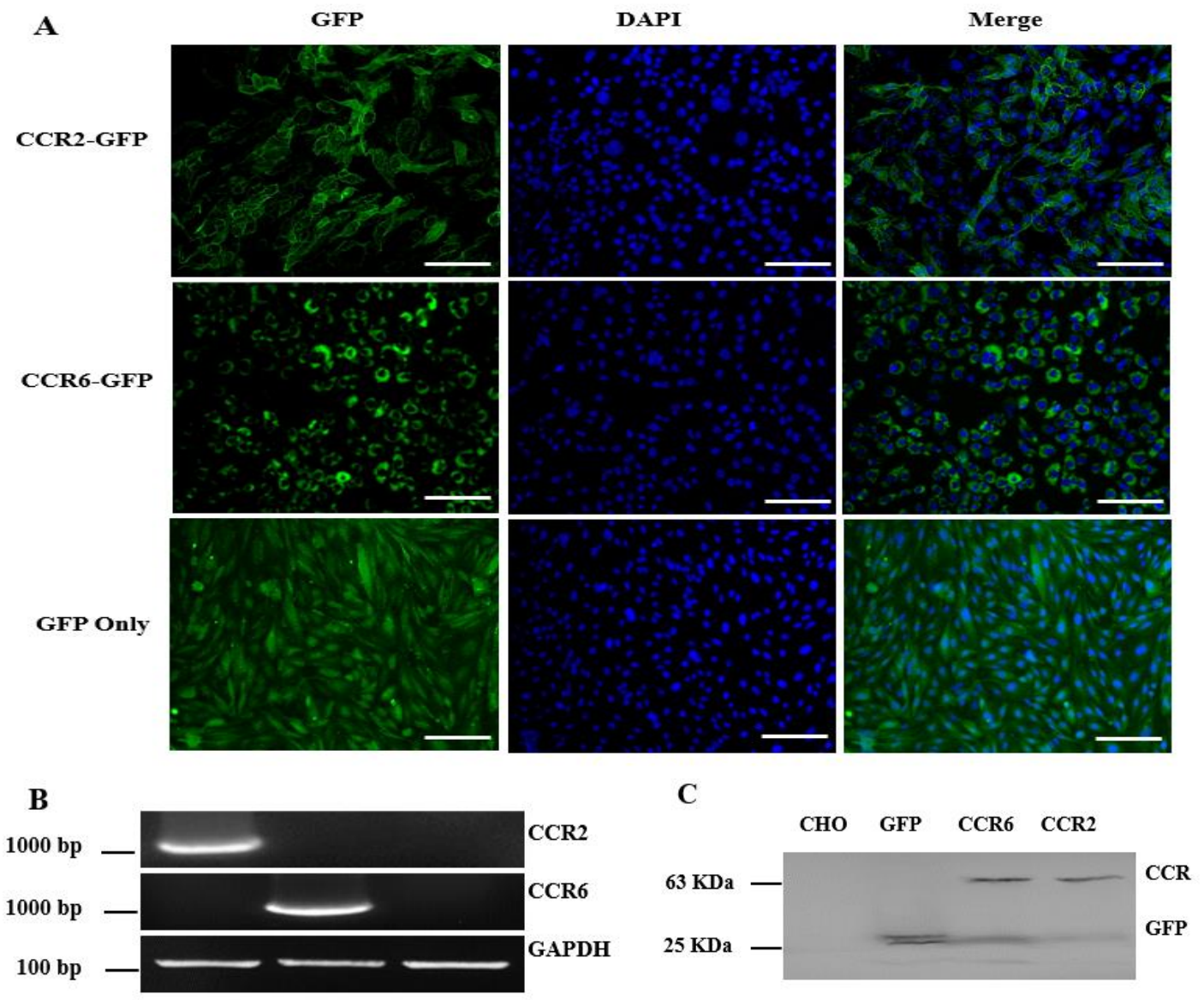

Figure 2-5. Expression of chicken CCR2 and CCR6 in CHO-K1 cells

(A) Fluorescence images of CCR2-GFP, CCR6-GFP and GFP proteins (green) expressing in $\mathrm{CHO}-\mathrm{K} 1$ cells. Transfected cells were fixed with methanol: acetone (v:v, 1:1), and nuclei were stained with $0.1 \mu \mathrm{g} / \mathrm{ml}$ DAPI (blue). (B) Gel electrophoresis of RT-PCR products of CCR2 (1,065 bp) and CCR6 (1,089 bp) derived from transfected CHO-K1 cells. GAPDH (125 bp) was included as a control. (C) Western-blotting analysis of the CCR2/CCR6-GFP fusion protein expressed in CHO-K1 cells, and CHO-K1 and only GFP expressing CHO$\mathrm{K} 1$ cells were included as negative and positive controls, respectively. The protein fractions were probed with a polyclonal goat anti-GFP primary antibody (1:1000) and HRP-coupled anti-goat secondary antibody (1:500) and visualized after color development with 4-CN and $30 \%$ hydrogen peroxide. Scale bar: $100 \mu \mathrm{m}$. 
$\mathbf{A}$

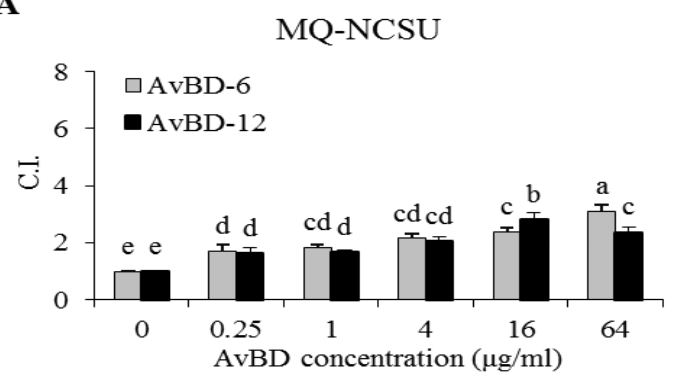

$\mathbf{C}$

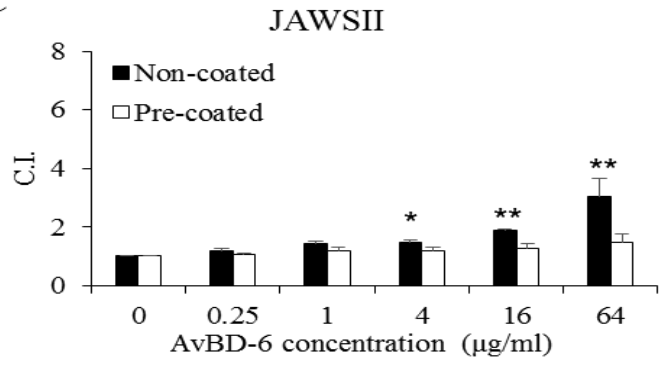

$\mathbf{E}$

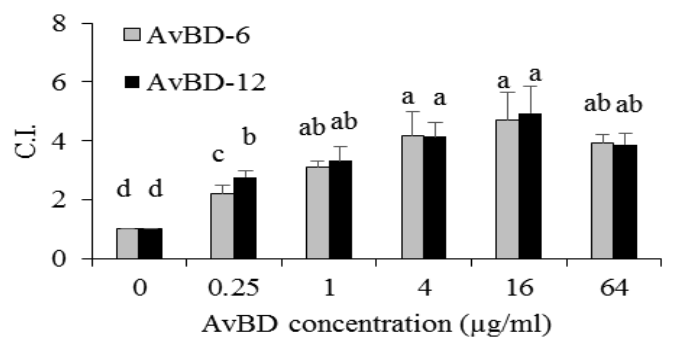

B

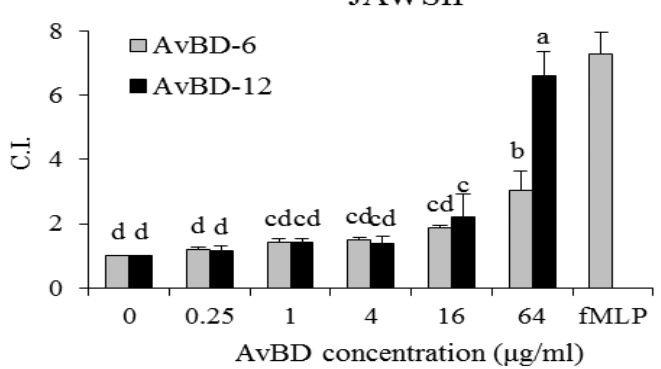

D

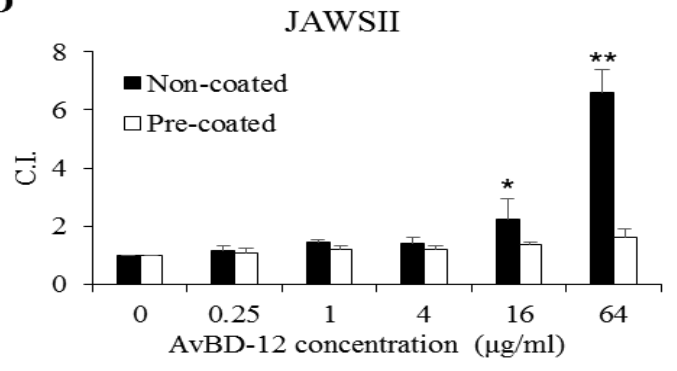

F CCR6-transfected CHO-K1 cells

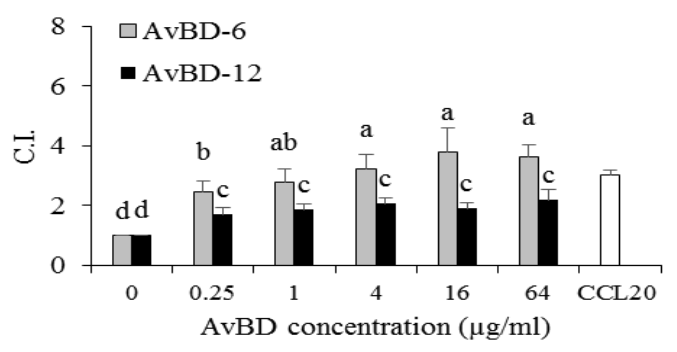

Figure 2-6. Chemotactic activity of AvBD-6 and AvBD-12

Migration of chicken macrophage MQ-NCSU cells (A), mouse immature dendritic JAWSII cells (B), AvBD-6 pretreated JAWSII cells (C), and AvBD-12 pretreated JAWSII cells (D), CCR2-positive CHO-K1 cells (E), CCR6-positive CHO-K1 cells (F). fLMP and CCL20 are used as positive control for dendritic cells and CCR6-transfected CHO-K1 cells, respectively. Chemotactic activities of AvBD-6 ( $\square$ ) and AvBD-12 ( $\square$ ) were expressed as chemotactic indexes (C.I.): = the number of migrated cells induced by AvBD/the number of migrated cells in response to chemotactic buffer. Data are presented as means of five independent experiments \pm SD $(\mathrm{n}=5)$. Different letters indicate significant difference $\left({ }^{*} p<\right.$ 0.05). Asterisks indicate significant difference $(* p<0.05, * * p<0.01)$. 


\section{The antimicrobial, chemotactic, and neutralizing LPS activities of reduced AvBDs}

To evaluate the role of disulfide bonds in antimicrobial and chemotactic activities, AvBDs were reduced with a thioredoxin system (Figure 2-7A). When defensins were fully reduced, the intramolecular disulfide bonds were broken and peptides became denatured which increased the overall hydrophobic surface area, compared to wild-type peptides with hydrophobic residues embedded inside. RP-HPLC analysis of AvBDs following treatment with various concentrations of thioredoxin revealed that AvBDs were completely reduced by $4 \mu \mathrm{M}$ thioredoxin as shown by the increased retention time with peak shifts (Figure 27A). The structural changes due to the removal of disulfide bonds were confirmed by farultraviolet circular dichroism (CD). Wild-type AvBD-6 and AvBD-12 displayed a broad negative band at $205 \mathrm{~nm}$ and a small negative shoulder at $216 \mathrm{~nm}$, indicating well-folded peptides with intracellular $\beta$-sheet conformation (Figure 2-7B). In contrast, reduced AvBD6 and AvBD-12 showed a significant negative signal around $195 \mathrm{~nm}$, indicating a random coil structure. In addition, reduced AvBD-12 also displayed a negative shoulder at about $216 \mathrm{~nm}$, which was likely caused by $\alpha$-helix of AvBD-12. These data indicated that treatment with the thioredoxin system broke the intramolecular disulfide bonds.

The reduced AvBD-6 and AvBD-12 showed antimicrobial activities similar to that of the wild-type AvBD-6 and AvBD-12, respectively (Figure 2-8A). However, reduced AvBDs lost their chemotactic effect on CCR2-CHO cells $(p<0.01$, Figure 2-8B). The reduction had a significant negative impact on the LPS-neutralizing activity of AvBD-12 (Figure 29C and 9D). For example, wild-type AvBD-12 at $32 \mu \mathrm{g} /$ well neutralized $74.48 \%$ of $E$. coli LPS (1EU/ml) whereas reduced AvBD-12 neutralized $28.78 \%$ E. coli LPS. A similar pattern was observed with $S$. Typhimurium LPS. 

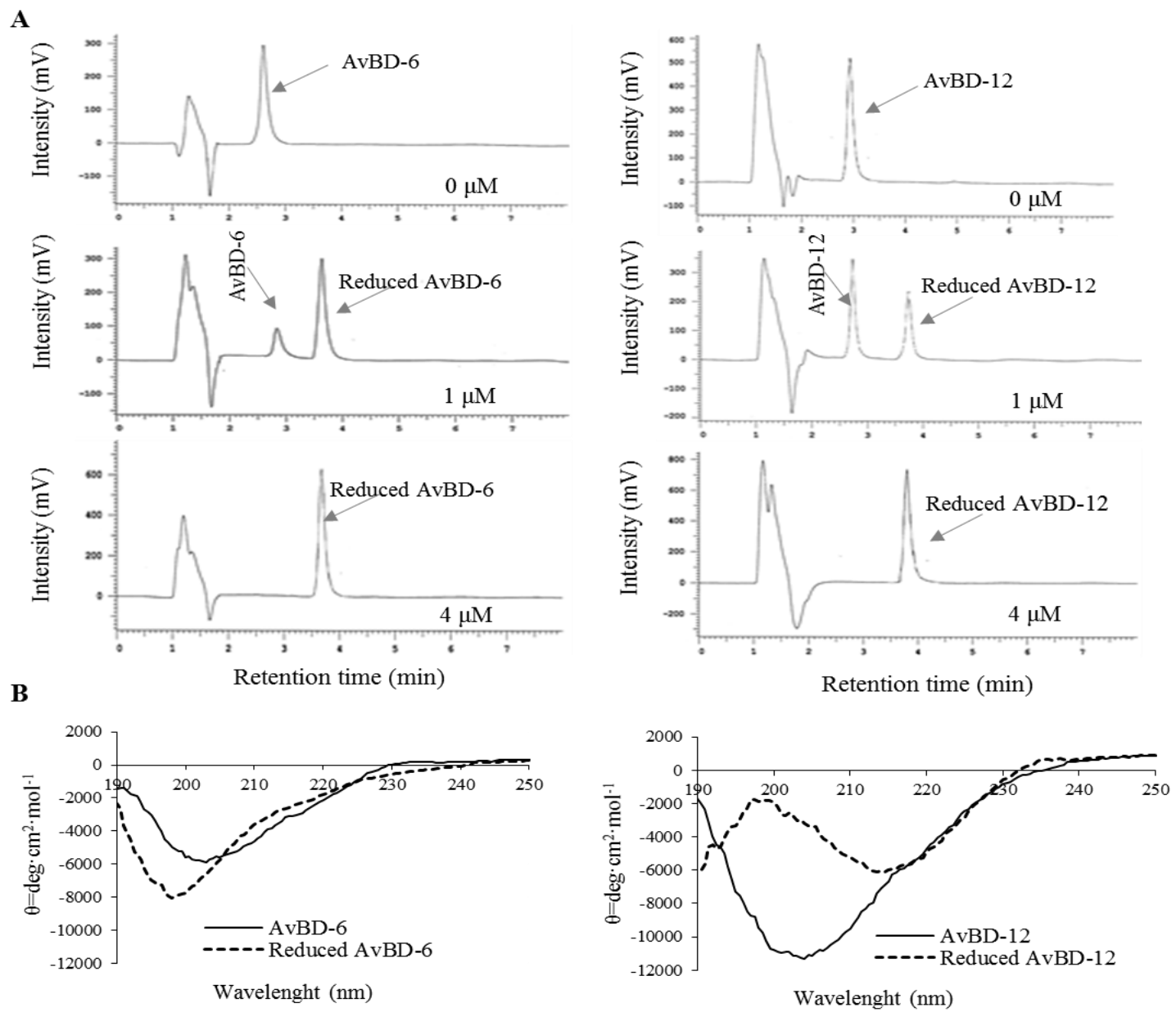

\section{Figure 2-7. Structure analysis of reduced and wildtype AvBD-6 and -12}

(A) RP-HPLC analysis of AvBD-6 and AvBD-12 reduced by thioredoxin system with various concentrations of thioredoxin $(0,1$, and $4 \mu \mathrm{M})$. Increased retention time and peak shifts indicated peptide unfolding and exposure of hydrophobic residues caused by thioredoxin treatment. Ten micrograms of peptides were completely reduced by treatment with $4 \mu \mathrm{M}$ thioredoxin. (B) Circular dichroism spectra of wild-type peptides (solid lines) and reduced peptides (dotted lines). Lines represent the average of six scans $(n=6)$. Wildtype peptides displayed intracellular $\beta$-sheet structure (signals at 205, $216 \mathrm{~nm}$ ). In contrast, reduced peptides showed a signal around $195 \mathrm{~nm}$, indicated random coil structure. Molar ellipticity $(\theta)=$ deg.cm ${ }^{2} \cdot \mathrm{dmol}^{-1}$. 

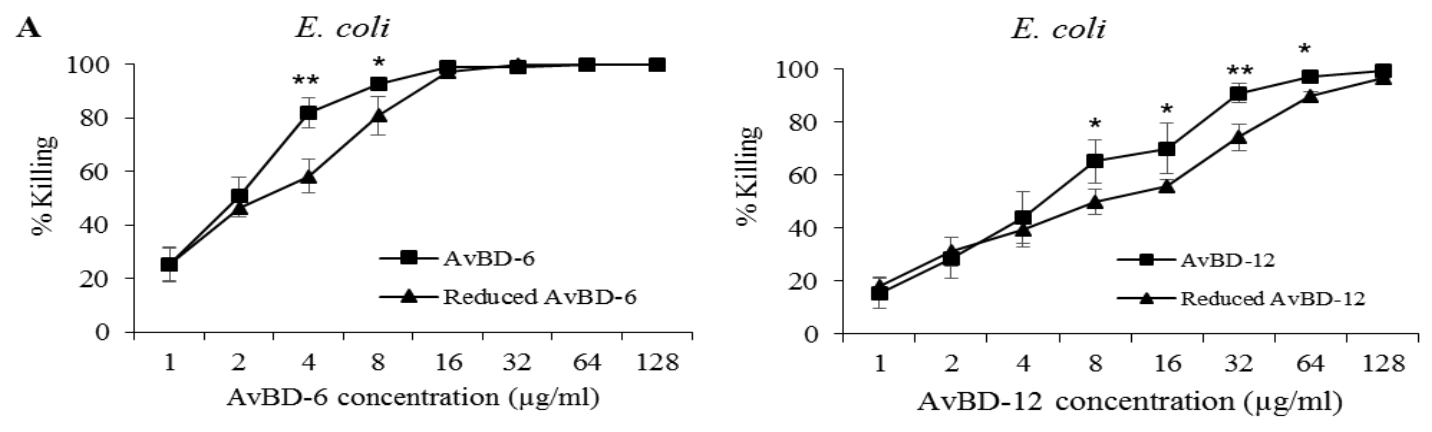

$S$. Typhimurium
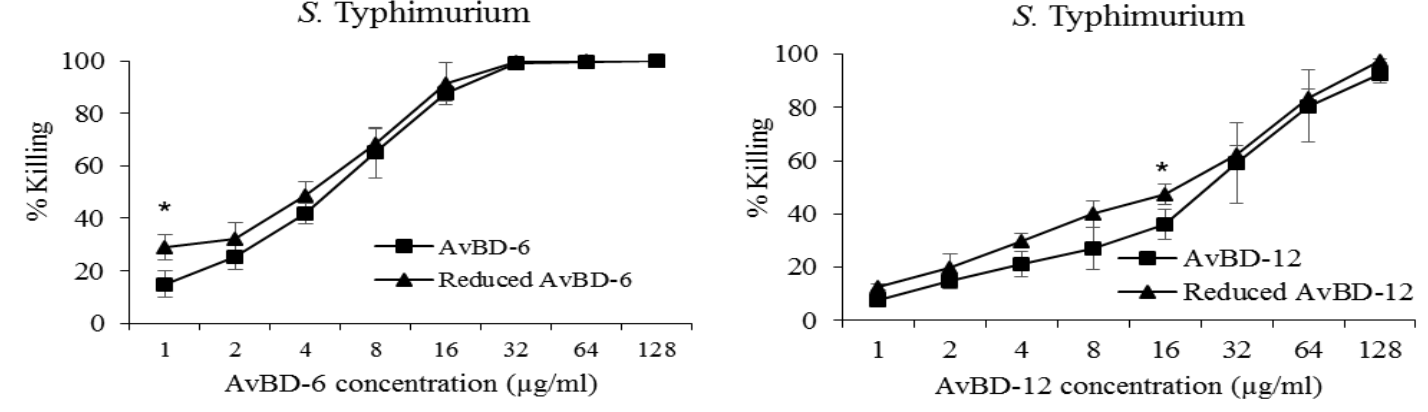

B CCR2-transfected CHO-K1 cells
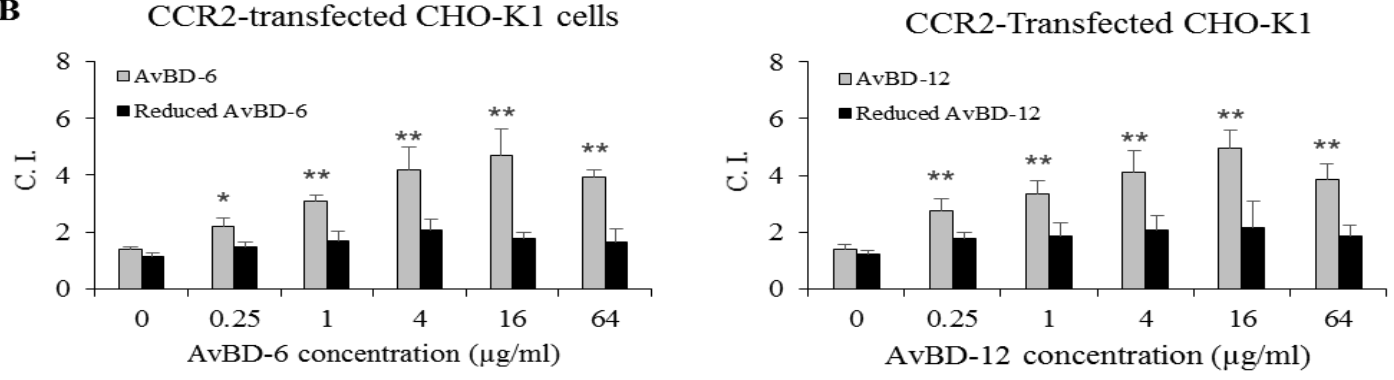

Figure 2-8. Antimicrobial and chemotactic activities of reduced AvBD-6 and -12

(A) Comparison of antimicrobial activity of reduced ( $\mathbf{\square})$ and wild-type ( $\boldsymbol{\Delta})$ AvBDs against E. coli and $S$. Typhimurium. The assay was repeated three times and data are presented as means \pm SD $(n=3)$. (B) Comparison of chemotactic activities of reduced and wild-type AvBDs. Chemotactic index (C.I.) = the number of migrated CCR-2 expressing CHO-K1 cells induced by AvBD / the number of migrated cells induced by the chemotactic buffer. Data present means \pm SD $(n=5)$. Asterisks indicate significant difference of chemotactic activity between wild-type and reduced AvBDs $\left(* p<0.05, *^{*} p<0.01\right)$. 
$\mathbf{A}$ E. coli LPS

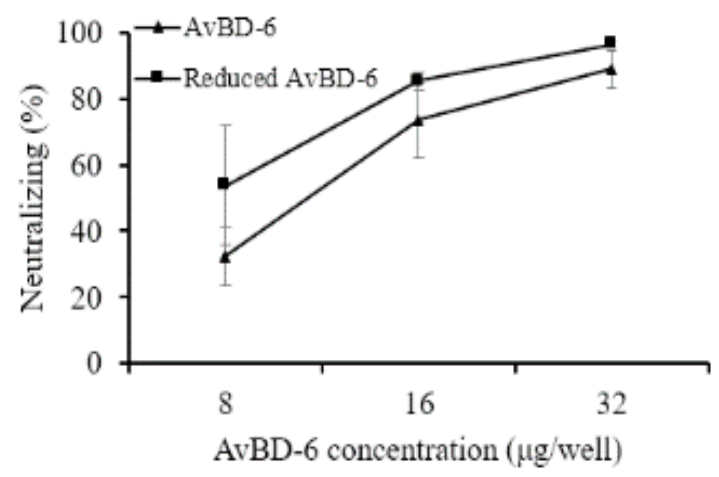

C

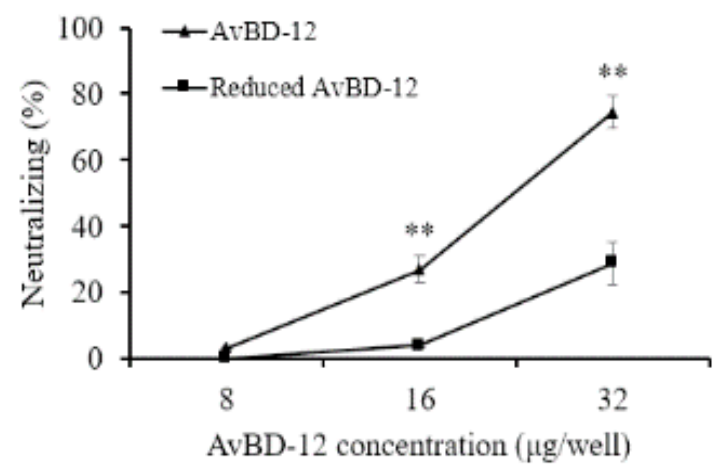

\section{B $\quad S$. Typhimurium LPS}

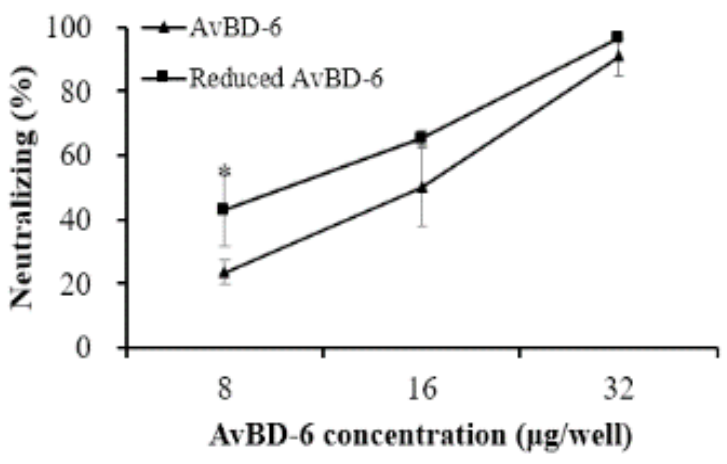

D

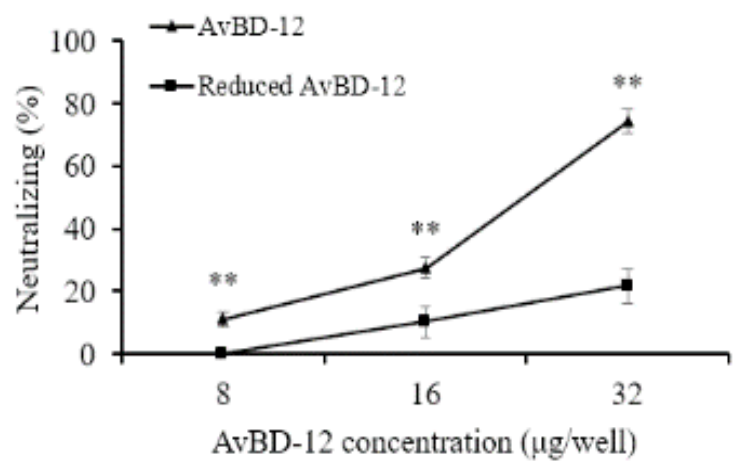

Figure 2-9. The LPS-neutralizing activity of reduced AvBD-6 and AvBD-12

Comparison of LPS-neutralizing activity of reduced ( $\boldsymbol{\square})$ and wild-type ( $\boldsymbol{\Delta}$ ) AvBDs. (A) Reduced AvBD-6 neutralizing E. coli O111: B4 LPS activity, (B) Reduced AvBD-6 neutralizing $S$. Typhimurium L6143 LPS activity, (C) Reduced AvBD-12 neutralizing $E$. coli O111: B4 LPS activity, (D) Reduced AvBD-12 neutralizing S. Typhimurium L6143 LPS activity. The assay was repeated three times and data are presented as means \pm SD $(n=3)$. Asterisks indicate significant difference of neutralizing LPS activity between wildtype and reduced AvBD-12 $(* p<0.05, * * p<0.01)$. 


\section{TEM observations}

Treatment of $S$. Typhimurium with wild-type AvBD-6 and AvBD-12 caused various structural changes at about $5 \%$ of bacterial cells, including fuzzy membrane, vacuole formation, membrane blebbing, and membrane shrinking (Figure 2-10A and 10B). Treatment of bacteria with reduced AvBD-6 and AvBD-12 caused only fuzzy membrane and leakage of cytoplasm as indicated by light staining in the cytosol (Figure 2-10C and 10D). The untreated bacteria displayed intact membrane, uniform cytoplasm without leakage of intracellular content (Figure 2-10E).

\section{Binding of AvBDs to bacterial genomic DNA}

The ability of AvBDs to bind to bacterial genomic DNA was analyzed by a gel retardation assay [22]. Wild-type AvBDs at a mass ratio of 4:1 (AvBD: DNA) retarded more than 50\% of $S$. Typhimurium genomic DNA migration (Figure 2-11). At a mass ration of 8:1 (AvBD: DNA), near complete retardation of genomic DNA migration by both AvBD-6 and AvBD12 was observed. BSA, as a negative control, had no effect at a mass ratio of 8:1 (BSA: DNA). At a mass ratio of 4:1 (AvBD: DNA), AvBD-6 was significantly more effective than AvBD-12 in retarding genomic DNA migration $(p<0.01)$. Reduced AvBDs were less able than their respective wild-type peptides to retard Salmonella genomic DNA migration. 


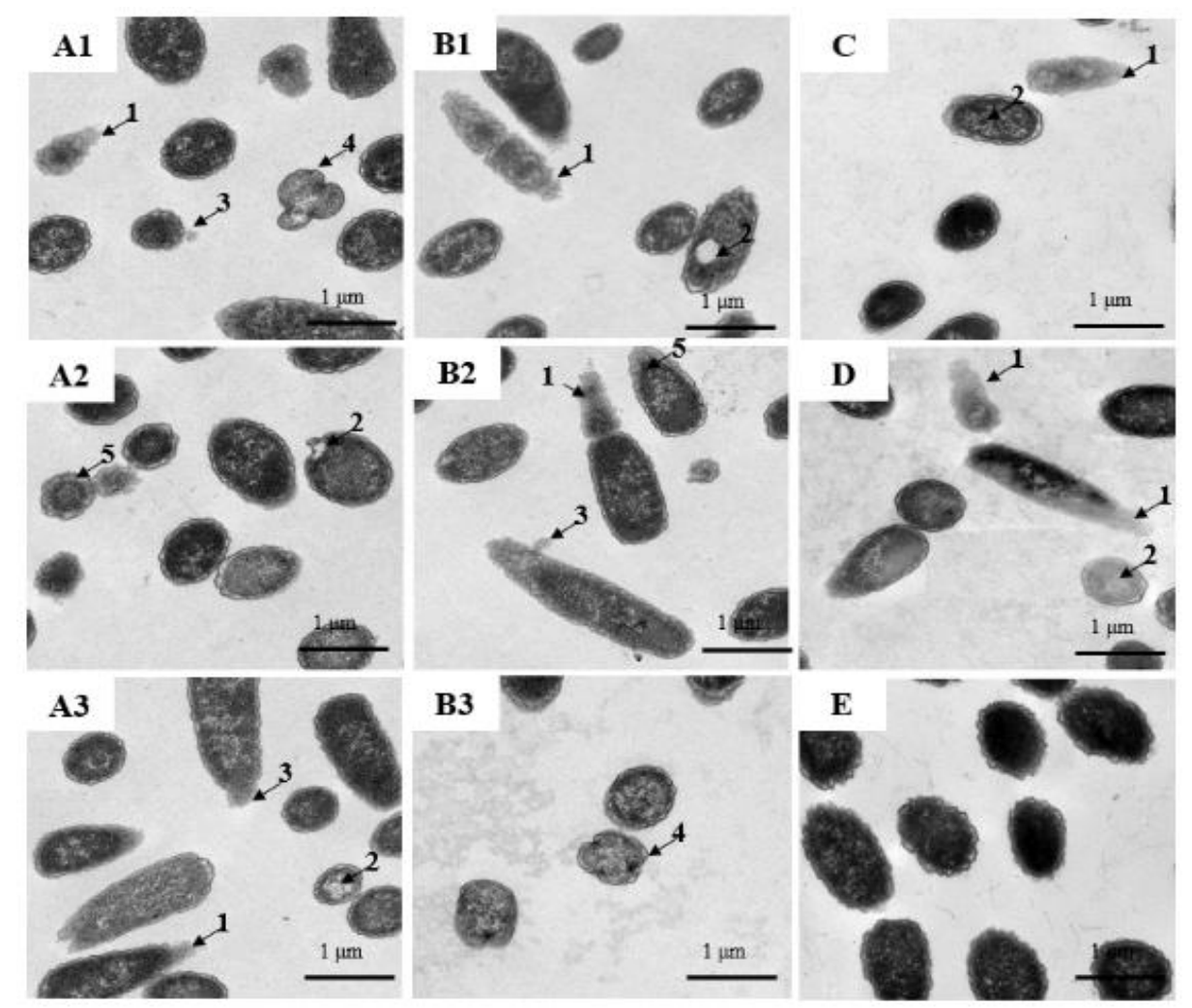

Figure 2-10. Transmission electron microscopy (TEM) analysis of AvBD-induced morphological changes of $S$. Typhimurium

Treatment of bacteria with wild-type and reduced AvBDs at $37^{\circ} \mathrm{C}$ for 30 min resulted in the fuzzy membrane (1), vacuole formation (2), membrane bleb (3), morphological change (4), and cytoplasm membrane shrinking (5). Bacteria were treated with AvBD-6 (A1-3), AvBD-12 (B1-3), reduced AvBD-6 (C), and reduced AvBD-12 (D). Untreated bacteria showed intact membrane, uniform cytoplasm (E). Figures are representatives of 10 images per treatment group. Approximately $29 \%$ of bacteria treated with wild-type AvBDs and $27 \%$ of bacteria treated with reduced AvBDs displayed ultrastructural changes. Scale bar: $1 \mu \mathrm{m}$. 

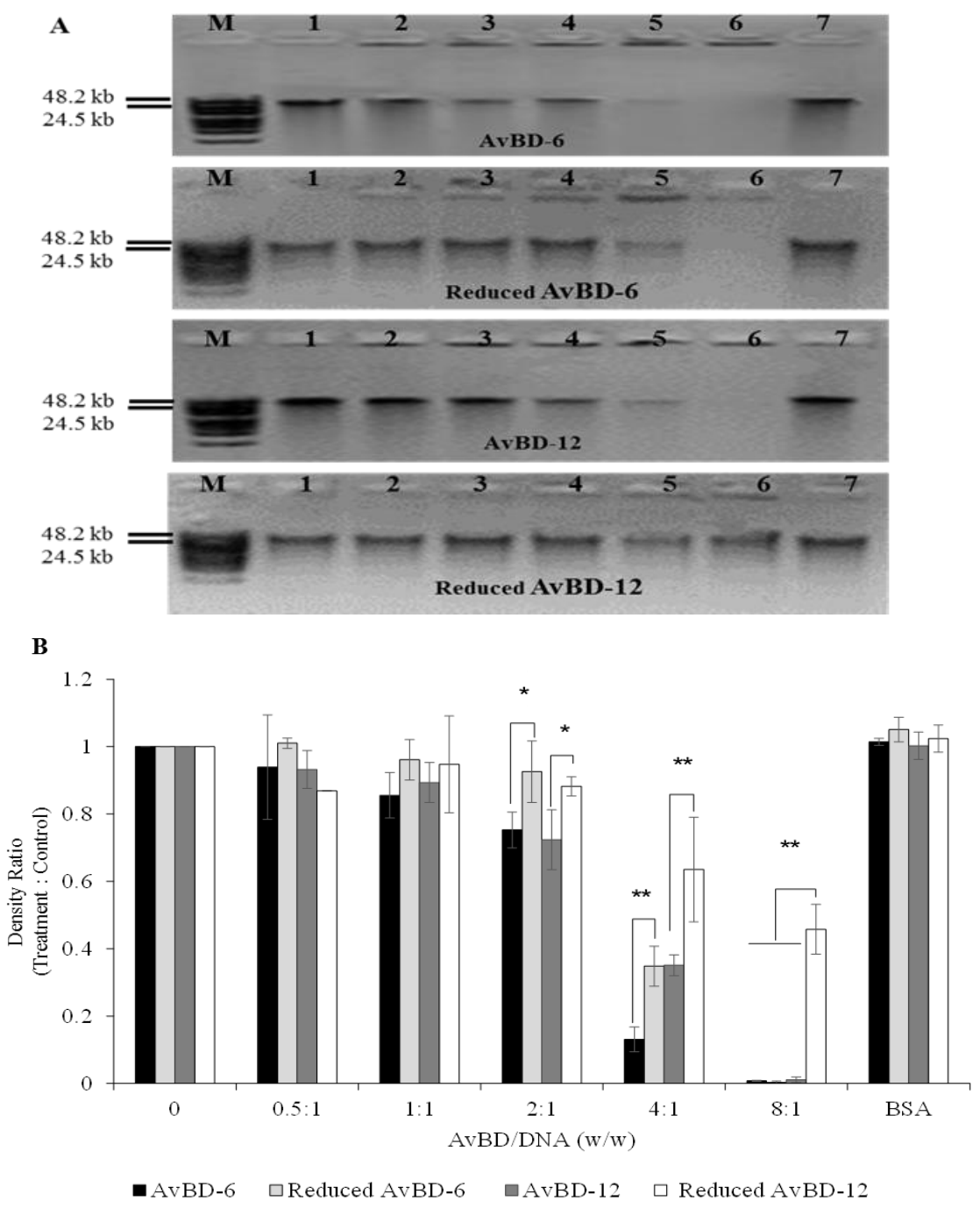

Figure 2-11. AvBD interaction with $S$. Typhimurium genomic DNA

(A) Gel retardation assay revealed the binding of wild-type and reduced AvBDs to $S$. Typhimurium genomic DNA. M: DNA molecular marker; lanes 1- 6: the mass ratios of DNA to AvBD were 1:0, 1:0.5, 1:1, 1:2, 1:4 and 1:8, respectively; lane 7: DNA: bovine serum albumin (BSA) at ratio of 1:8. (B) Densitometric analysis of migrated DNA by ImageJ software. Density ratio between treatment and control (lane 1) groups were calculated. Displayed values are means \pm SD $(n=3)$. Asterisks indicate significant difference $(* p<0.05, * * p<0.01)$ between wild-type and reduced AvBDs. 


\section{Discussion}

To understand the molecular mechanisms by which AvBDs contribute to host innate and acquired immunity, we have studied the transcriptional profiles of AvBD genes and characterized the antimicrobial activities of AvBDs with different net positive charges and tissue expression profiles $[40,111]$. In the present study, we chemically synthesized the mature peptides of AvBD-6 and AvBD-12 and characterized the roles of peptide charge and intramolecular disulfide bonds in various biological functions. Our data indicated that synthetic AvBD-6 and AvBD-12 were highly effective in killing E. coli, S. Typhimurium, and $S$. aureus under low salt condition. Compared to recombinant AvBDs, synthetic AvBDs demonstrated a potent antimicrobial activity against $S$. Typhimurium which was minimally susceptible to recombinant AvBDs in our previous study [57]. The discrepancy might be related to peptide folding because synthetic but not recombinant AvBDs were subjected to oxidative folding under optimal conditions. Consistent with our previous findings with recombinant AvBDs, the antimicrobial activity of AvBD-6 with a net positive charge of +7 was significantly higher than that of AvBD-12 with a net positive charge of +1. The antimicrobial potency of both AvBDs against E. coli and $S$. Typhimurium and AvBD-12 against $S$. Typhimurium was negatively affected by increased salt concentration which was consistent with findings from previous investigations of cationic antimicrobial peptides $[8,11,25,57]$. However, increasing salt concentration had no impact on the bactericidal activity of AvBD-12 against $S$. aureus due to a lower charge or other killing mechanisms that were utilized to kill Gram-positive and Gram-negative bacteria. In this study, we also found that the antimicrobial effect of AvBDs was inversely associated with bacterial concentration, suggesting that a critical molecular ratio of AvBD: bacteria may 
be needed to damage the microbial membrane. The antimicrobial activity of AvBDs (32 $\mu \mathrm{g} / \mathrm{ml}$ ) nearly diminished when bacterial concentration was increased from $10^{5} \mathrm{CFU} / \mathrm{ml}$ $\left(\mathrm{CFU}: \mathrm{AvBD}\right.$ molecule ratio $\left.=1: 4 \times 10^{2}\right)$ to $10^{9} \mathrm{CFU} / \mathrm{ml}(\mathrm{CFU}: \mathrm{AvBD}$ molecule ratio $=1: 4$ $\left.\times 10^{6}\right)$. The present study also evaluated the antimicrobial activities of AvBDs against clinical isolates of ESBL-positive E. coli and K. pneumoniae and methicillin-resistant $S$. pseudintermedius with the measurement of MICs. Although both AvBDs showed antimicrobial activity against ESBL-positive $E$. coli and $K$. pneumoniae, the MIC values ranged from 128 to $256 \mu \mathrm{g} / \mathrm{ml}$, as compared to 4 to $64 \mu \mathrm{g} / \mathrm{ml}$ when the assays were conducted under low salt condition. It has been reported that defensins interact with bacterial membrane electrostatically and thereby destroy the integrity of the membrane to kill the bacteria [10]. Therefore, it is important to determine whether these peptides can disrupt the host cellular membrane. In the present study, AvBDs at a relatively high concentration $(256 \mu \mathrm{g} / \mathrm{ml})$ did not affect the viability of avian and mammalian cell lines examined. Our research results indicate that AvBDs may be a potential alternative therapeutic agent for infections caused by ESBL-producing bacteria if their sensitivity to salt can be modified.

It has been reported that the presence of disulfide bonds was not essential for bovine BNDB-2 and BNDB-12 or human hBD3 to exert their antimicrobial function $[64,121-$ 124]. To investigate the structure-function relationship of AvBDs, we treated AvBDs with the thioredoxin reductase to break the disulfide bonds and confirmed the reduction of AvBDs by RP-HPLC and CD spectrum [125-127]. Reduced AvBDs showed slightly reduced potency against $E$. coli which was in line with a previous report that AvBD-6 
without the three disulfide bonds expressed in Pichia pastoris had antimicrobial activity against S. aureus and Bacillus subtilis [128].

As a major component of Gram-negative bacteria, LPS maintains the structural integrity of bacteria and causes septic shock or inflammatory responses in the host [129]. It was previously shown that human defensin beta 123 (DEFB123) and recombinant human DEFB114 could inhibit LPS-mediated inflammation [130, 131]. The electrostatic interactions between defensins and negatively charged LPS are prerequisite for the binding of peptides to Gram-negative bacteria and subsequent membrane damaging activities. Therefore, any factors (such as cationic salt concentration) interfering with the electrostatic attraction could inhibit defensins' antimicrobial functions [132]. In the present investigation, AvBD-6 with a higher net positive charge was more able than AvBD-12 to neutralize LPS, but LPS-neutralizing capacity of neither AvBD was affected by increased $\mathrm{NaCl}$ concentration. In fact, both AvBDs were fully functional at near physiological concentration of $\mathrm{NaCl}(0.8 \%, 137 \mathrm{mM})$. However, the LPS-neutralizing ability correlated with the net positive charges of AvBDs, suggesting that electrostatic attraction is an essential, but not the only factor that affects the interaction between AvBD and LPS. This was further supported by the fact that reduced AvBDs having the same net positive charges as their respective wildtype peptides were less able to neutralize LPS, specifically for AvBD-12.

Mammalian defensins can interact with cell membrane receptors to influence diverse cellular processes including antigen presentation, chemotaxis, and cytokine release [37, 133]. Both AvBDs demonstrated mild chemotactic activities for MQ-NCSU, and AVBD12 at a relatively high concentration had strong chemoattractant effect on murine immature 
dendritic cells. Pretreatment JAWSII cells with AvBD blocked AvBD-induced cell migration $(p<0.05)$, suggesting that AvBDs interacted with murine dendritic cell membrane component(s), likely chemokine receptors which are highly conserved across species $[134,135]$. We then determined if CCR2 and CCR6 were the receptors for AvBDs as indicated previously for hBD1-3 and mBD2 and mBD14 [64, 104-106]. Both AvBD-6 and AvBD-12 displayed strong chemotactic effect on CCR2-positive CHO-K1 cells whereas only AvBD-6 induced moderate chemotaxis of CCR6-positive CHO-K1 cells. CCR6 as a G-protein-coupled receptor with seven transmembrane domain is expressed by memory $\mathrm{T}$ cells, B cells, and DCs and involved in recruiting leukocytes to sites of inflammation [136]. In the present study, fusion with GFP changed the location of CCR6, which might have affected the chemotactic migration of CCR6-positive CHO-K1 cells. We conducted several independent transfection assays with different clones of CCR6 plasmids, all of which resulted in the primary expression of CCR6-GFP in the nuclear membrane. Nonetheless, our data show that AvBDs are broad-spectrum chemoattractant molecules for avian and mammalian immune cells.

The dual antimicrobial mechanism involving targeting microbial membrane and nucleic acids and enzymes have been reported [137-142]. We further determined the role of disulfide bonds of AvBDs in damaging bacterial membrane and binding to genomic DNA. Treatment of $S$. Typhimurium with AvBDs resulted in the fuzzy membrane, loss of cytoplasmic content, cytoplasmic membrane shrinkage, and morphological change (Figure 2-10A and 10B) whereas reduced AvBDs caused only fuzzy membrane and leakage of intracellular content. Our data suggest that wild-type AvBDs with conserved disulfide bonds not only disrupted bacterial membrane but also interfered with cell division and other 
intracellular functions that cause morphological changes. In contrast, reduced AvBDs exert their bactericidal function mainly through a membrane-lytic mechanism(s). Gel retardation assay ascertained that wild-type AvBDs were more able than reduced AvBDs to bind bacterial genomic DNA, potentially interfering with transcription and translation of affected bacteria.

\section{Conclusions}

AvBD-6 and AvBD-12 exhibited strong antimicrobial activities against E. coli, S. Typhimurium, and $S$. aureus under low salt condition. The antimicrobial activity was positively correlated with the net positive charges of AvBDs, inversely correlated with bacterial concentration, strongly inhibited by $\mathrm{NaCl}$ at physiological concentration (150 $\mathrm{mM}$ ), and minimally dependent on the intramolecular disulfide bonds. The LPSneutralizing activity of AvBDs was also positively correlated with the net positive charge, dependent on disulfide bonds (for AvBD-12), and unaffected by $\mathrm{NaCl}$ concentration. The chemotactic activity required the tertiary structure of AvBDs but not directly related to the peptide charge.

Functional characterization of two different AvBDs suggests that different mechanisms could be involved in their actions against different microbial pathogens and microbial products (such as LPS). Data from the present investigation provide the theoretical basis for future application of AvBDs or their analogs as therapeutic agents for bacterial infections and LPS-induced shock, as well as vaccine adjuvants for avian and mammalian species. 


\title{
CHAPTER 3: Novel Synthetic Analogs of Avian $\beta$-Defensin-12: the
}

\section{Roles of Charge, Hydrophobicity, and Disulfide Bonds in Biological}

\section{Functions}

Published, BMC Microbiology (2017) 17:43.

\begin{abstract}
Avian $\beta$-defensins (AvBD) possess broad-spectrum antimicrobial, LPS neutralizing and chemotactic properties. AvBD-12 is a chemoattractant for avian immune cells and mammalian dendritic cells (JAWSII) - a unique feature that is relevant to the applications of AvBDs as chemotherapeutic agents in mammalian hosts. To identify the structural components essential to various biological functions, we have designed and evaluated seven AvBD analogs. In the first group of analogs, the three conserved disulfide bonds were eliminated by replacing cysteines with alanine and serine residues, peptide hydrophobicity and net positive charge were increased by changing negatively charged amino acid residues to hydrophobic (AvBD-12A1) or positively charged residues (AvBD12A2 and AvBD-12A3). All three analogs in this group showed improved antimicrobial activity, and AvBD-12A3 with a net positive charge of +9 , the hydrophobicity of $40 \%$, and a predicted CCR2 binding domain, was the most potent antimicrobial peptide. AvBD-12A3 also retained more than $50 \%$ of wild-type chemotactic activity. In the second group of analogs (AvBD-12A4 to AvBD-12A6), one to three disulfide bonds were removed via substitution of cysteines with isosteric amino acids. Their antimicrobial activity was compromised and chemotactic activity abolished. The third type of analog was a hybrid that had the backbone of AvBD-12 and four amino acid residues AvBD-6. The antimicrobial and chemotactic activities of the hybrid resembled that of AvBD-6 and
\end{abstract}


AvBD-12, respectively. In conclusion, while the net positive charge and charge distribution have a dominating effect on the antimicrobial potency of AvBDs, the three conserved disulfide bonds are essential to the chemotactic property and the maximum antimicrobial activity. Analog AvBD-12A3 with a high net positive charge, a moderate degree of hydrophobicity, and a CCR2-binding domain can serve as a template for the design of novel antimicrobial peptides with chemotactic property and salt resistance.

\section{Background}

The rapid emergence of antimicrobial resistance poses a major global threat to public health and the economy $[3,143]$. Excessive use and misuse of antibiotics in medicine and food production contribute to the rise of drug-resistant pathogens [144, 145]. Control and prevention of antibiotic resistance call for holistic strategies including the judicious use of antimicrobials, effective diagnostic tools, and novel therapeutic agents that are less likely to trigger resistance [146]. Beta-defensins are small, cationic, antimicrobial peptides found in different living organisms $[11,39,55,96]$. These endogenous peptides constitute the first line of innate defense against pathogenic bacteria, fungi, and viruses [45, 133]. Betadefensins possess various biological properties, including broad-spectrum microbicidal activity, neutralization of LPS, activation of macrophages and dendritic cells, chemoattraction of dendritic cells, monocytes, and T-lymphocytes to the site of infection $[45,55,103]$. The microbicidal activity of defensins is achieved mainly through initial electrostatic attraction between positively charged amino acid residues of the peptides and negatively charged microbial surface components and subsequent microbial membrane damage which is followed by interacting with intracellular targets $[9,25,98]$. The chemotactic function of $\beta$-defensins is mediated by CC-chemokine receptors, such as 
CCR2 and CCR6 [64, 104, 106]. These natural antimicrobial peptides with complex mechanisms of action represent potentially a novel class of antimicrobials [98, 147]. However, several challenges must be addressed in order to develop $\beta$-defensins for therapeutic use which include retaining the biological activities under physiological conditions and the ease of production and purification of recombinant or synthetic peptides $[57,148]$

Most defensin peptides characterized to date have a net positive charge, ranging from +2 to +9 , and hydrophobicity of approximately $30-50 \%$ [25]. It has been reported that the three conserved disulfide bonds are required for the chemotactic function, but not the antimicrobial activity [64, 121-124]. Data from our laboratory showed that reduced (or linear form) AvBDs were fully active against bacterial pathogens whereas AvBDs without correctly folded disulfide bonds were not $[57,59]$. To further understand the structural and functional characteristics of AvBDs, seven analogs were designed by replacing the negatively charged residues (D and E) and/or cysteines (C) with either positively charged residues $(\mathrm{H}, \mathrm{K}$, and $\mathrm{R})$, hydrophobic residues (A, I, L, and $\mathrm{V}$ ) or isosteric amino acids (Abu). The antimicrobial and chemotactic activities and salt-resistance of the analogs, as well as their wild-type parent peptides, have been evaluated.

\section{Methods and Materials}

\section{Design and Synthesis of Peptides}

The three-dimensional structures of AvBDs were predicted by using the I-TASSER program (http://zhanglab.ccmb.med.umich.edu/I-TASSER). The distribution of positively charged amino acids was evaluated using PyMOL (https://www.pymol.org/). Group 1 analogs including AvBD-12A1, A2 and A3 were linear peptides, in which the six cysteines 
$\left(C^{1} C^{2} C^{3} C^{4} C^{5} C^{6}\right.$ or $\left.C 5 C 12 C 17 C 27 C 34 C 35\right)$ were replaced with structurally similar amino acid residues (alanine and serine) as follows: AvBD-12A1: A5A12A17A27A34A35, AvBD-12A2: S5S12S17A27A34A35, and AvBD-12A3: A5A12A17S27S34S35. Additional modifications were introduced to these analogs to alter peptide hydrophobicity and charge. In AvBD-12A1, the four negatively charged amino acid residues (D3D8E21E29) were replaced with one polar and three hydrophobic amino acid residues (H3V8L21I29). In AvBD-12A2 and AvBD-12A3, D3D8E21E29 were substituted with positively charged residues R3K8K21R29. Group 2 analogs including AvBD-12A4, A5, and A6 had reduced number of disulfide bonds without any additional modifications. To remove disulfide bonds, the cysteine residues $\left(\mathrm{C}^{1} \mathrm{C}^{2} \mathrm{C}^{3} \mathrm{C}^{4} \mathrm{C}^{5} \mathrm{C}^{6}\right.$ or $\left.\mathrm{C} 5 \mathrm{C} 12 \mathrm{C} 17 \mathrm{C} 27 \mathrm{C} 34 \mathrm{C} 35\right)$ of AvBD-12 were replaced with isosteric $\alpha$-aminobutyric acids (Abu, $\mathrm{U}$ ) to create AvBD12A4 (U5C12C17C27U34C35), A5 (U5U12C17U27U34C35), and A6 (U5U12U17U27 U34U35). Group 3 included a single hybrid peptide, namely AvBD-12/6. This analog was designed using the backbone of AvBD-12, in which the negatively charged amino acid residues (D3D8E21E29) of AvBD-12 were replaced with amino acids (H3Q8Y21S29) of AvBD-6 at the corresponding positions. The hydrophobicity and charge of AvBD analogs at neutral $\mathrm{pH}$ were calculated using Peptide 2.0 (http://peptide2.com) and Peptide property calculator (PepCalc.com), respectively.

All peptides were custom synthesized using the standard solid phase 9fluorenylmethoxycarbonyl (Fmoc) method and purified by reverse phase highperformance liquid chromatography (RP-HPLC) (Lifetein, Hillsborough, NJ). The analogs AvBD-12A4, -A5, AvBD-12/6, AvBD-6 and AvBD-12 with varying numbers of cysteine residues were subjected to oxidative folding as described previously [64]. Electrospray 
ionization mass spectrometry (ESI-MS) was performed to confirm the correct formation of disulfide bonds between $\mathrm{Cys}^{1}-\mathrm{Cys}^{5}, \mathrm{Cys}^{2}-\mathrm{Cys}^{4}$, and $\mathrm{Cys}^{3}-\mathrm{Cys}^{6}$. The purity of the synthetic AvBD analogs was over $98.5 \%$ as verified by liquid chromatography-mass spectrometry (LC-MS) (Lifetein, Hillsborough, NJ).

\section{Antimicrobial activity of AvBD analogs}

Escherichia coli (ATCC 25922), Pseudomonas aeruginosa (ATCC 27853), Salmonella Typhimurium (ATCC 14028) and Staphylococcus aureus (ATCC 29213) were used to assess the antimicrobial activity of AvBD analogs. All bacterial strains were grown on LB agar plates or TSA with $5 \%$ Sheep Blood (Thermo Fisher Scientific) plates at $37{ }^{\circ} \mathrm{C}$. Antimicrobial activity was determined by a colony counting assay $[57,59]$. The procedure was same as the antimicrobial assay in Chapter 2. To assess the resistance of AvBD analogs to $\mathrm{NaCl}$, antimicrobial assays were carried out in the presence of $5 \mathrm{mM}, 50 \mathrm{mM}, 100 \mathrm{mM}$ or $150 \mathrm{mM} \mathrm{NaCl}$. All assays were performed in triplicate.

\section{Minimum inhibitory concentrations of AvBD analogs}

The minimum inhibitory concentrations (MIC) of AvBDs were determined according to the guidelines of the Clinical and Laboratory Standards Institute (CLSI) [117, 118]. The Muller Hinton II broth used in standard MIC assays contained 20-25 mg/L of calcium and 10-12.5 mg/L of magnesium. Modified MICs of AvBDs were also determined under a lowsalt and low-nutrient condition by using $100 \times$ diluted Muller Hinton II broth containing $0.2 \mathrm{mg} / \mathrm{L}$ of calcium, $0.1 \mathrm{mg} / \mathrm{L}$ magnesium and $5 \mathrm{mM}(292 \mathrm{mg} / \mathrm{L}) \mathrm{NaCl}$. MIC obtained under the low-salt and low-nutrient condition was referred to as MIC-ls. AvBD peptides were two-fold serially diluted ( 2 to $256 \mu \mathrm{g} / \mathrm{ml}$ ) in a 96-well microtiter plate. An equal volume $(\mu 1)$ of bacterial suspension was added to each well of the plate. The final bacterial 
concentration in the wells was $5 \times 10^{5} \mathrm{CFU} / \mathrm{ml}$. The plate was incubated at $37{ }^{\circ} \mathrm{C}$ for $24 \mathrm{~h}$ and the lowest concentration that completely prevented visible bacteria growth was recorded. To complement MIC-ls assays, the minimum bactericidal concentrations (MBC) were evaluated by sub-culturing the contents of the first two clear wells obtained in the MIC-ls assay onto LB agar plates. All assays were conducted in triplicate. The lowest peptide concentration inhibiting more than $99 \%$ of bacterial growth was defined as MBCls. AvBD analogs were regarded as bactericidal if the $\mathrm{MBC}$ was no more than four times the MIC [149].

\section{Cell cytotoxicity assay}

Chicken macrophage cell lines MQ-NCSU and HD11 were maintained in RPMI-1640 media supplemented with $10 \%$ fetal bovine serum (FBS), $2 \%$ chicken serum, $100 \mathrm{U} / \mathrm{ml}$ penicillin and $100 \mu \mathrm{g} / \mathrm{ml}$ streptomycin (Sigma-Aldrich) at $37{ }^{\circ} \mathrm{C}$ in humidified air with $5 \%$ $\mathrm{CO}_{2}$. CHO-K1 cells were cultured in the same media without $2 \%$ chicken serum. For CCR2-transfected CHO-K1 cells, the medium was supplemented with $500 \mu \mathrm{g} / \mathrm{ml} \mathrm{G418}$ was added (Sigma-Aldrich) [59]. Murine immature dendritic cell line JAWSII (ATCC CRL-11904 ${ }^{\mathrm{TM}}$ ) was cultured in Alpha minimum essential medium containing $4 \mathrm{mM} \mathrm{L-}$ glutamine, $1 \mathrm{mM}$ sodium pyruvate, $5 \mathrm{ng} / \mathrm{ml}$ murine Granulocyte macrophage-colony stimulating factor (GM-CSF), 20\% FBS, $100 \mathrm{U} / \mathrm{ml}$ penicillin and $100 \mu \mathrm{g} / \mathrm{ml}$ streptomycin at $37{ }^{\circ} \mathrm{C}$ in humidified air with $5 \% \mathrm{CO}_{2}$. The cell cytotoxicity was determined using the MTT (3-(4, 5-dimethylthiazol-2-yl)-2, 5-diphenyltetrazolium bromide metabolic activity assay according to the manufacturer's instruction (Thermo Fisher Scientific). Briefly, cells $\left(5 \times 10^{3}\right.$ cells/well) were seeded in $96-$ well tissue culture plates, incubated overnight and treated with AvBDs at concentrations of 4, 16, 64 and $256 \mu \mathrm{g} / \mathrm{ml}$ for 4, 12, 24 and $48 \mathrm{~h}$ at 
$37^{\circ} \mathrm{C}$. After treatment, $20 \mu \mathrm{l}$ of $12 \mathrm{mM}$ MTT solution was added to each well. The plate was incubated for $4 \mathrm{~h}$ and read at $540 \mathrm{~nm}$. Viability was expressed as a percentage of viable cells relative to the untreated control. The experiments were performed in triplicate.

\section{Chemotaxis assay}

Migration of JAWSII and CCR2-CHO-K1 cells in response to AvBD-12 analogs was determined using a 48-well microchemotaxis chamber technique as previously described [119]. Chemotaxis buffer (Minimum Essential Medium containing 0.1\% BSA, 100U/ml penicillin, and $100 \mu \mathrm{g} / \mathrm{ml}$ streptomycin) and bacterial peptide $\mathrm{N}$-formyl-methionyl-leucylphenylalanine (fMLF, Sigma-Aldrich) were included as negative and positive controls, respectively. The results were presented as chemotactic indexes (C.I.). C.I. $=$ number of migrated cells induced by AvBDs / number of migrated cells induced by the chemotactic buffer. The assay was repeated five times.

\section{Scanning Electron Microscopy (SEM)}

SEM was performed according to the procedure described by Cobo et al. [150]. S. Typhimurium was cultured in Mueller Hinton (MH) broth to mid-log phase and harvested by centrifugation at $5000 \mathrm{~g}$ for $10 \mathrm{~min}$. Cell pellets were washed twice with $10 \mathrm{mM}$ PBS and resuspended at a final number of $10^{8} \mathrm{CFU}$. The cell suspension was incubated with $1 \times$ MIC-ls of AvBD-12A3, AvBD-12/6, wild-type AvBD-6 and AvBD-12 at $37^{\circ} \mathrm{C}$ for 30 min. Bacterial pellets were fixed in $500 \mathrm{ml}$ of $2.5 \%(\mathrm{v} / \mathrm{v})$ glutaraldehyde in $0.2 \mathrm{M}$ cacodylate buffer at $4{ }^{\circ} \mathrm{C}$ overnight, washed twice with $0.2 \mathrm{M}$ cacodylate buffer and dehydrated through ethanol gradient $(30 \%, 50 \%, 70 \%, 90 \%, 100 \%$ and again $100 \%)$ for $15 \mathrm{~min}$ in each gradient. The samples were transferred into a mixture $(1: 1, \mathrm{v} / \mathrm{v})$ of ethanol 
and tertiary butanol and then pure tertiary butanol for 20 min each. After gold coating, the specimens were observed using a scanning electron microscope (Hitachi S-4700, Japan).

\section{Statistical analysis}

Data were presented as the means \pm standard deviation (SD). Differences between groups were analyzed using the one-way analysis of variance (ANOVA) followed by Duncan's test for multiple comparisons using software SPSS version 19.0 (IBM Corp., Armonk, NY). Differences at $p<0.05$ level were considered statistically significant, and at $p<0.01$ level were considered extremely significant.

\section{Results}

\section{Structural characteristics of AvBD-6 and AvBD-12 and their analogs}

Substitutions of $C^{1} C^{2} C^{3} C^{4} C^{5} C^{6}$ (or $C 5 C 12 C 17 C 27 C 34 C 35$ ) by A5A12A17A27A34A35 (AvBD-12A1) not only eliminated the three disulfide bonds but also elevated the hydrophobicity from 33\% (AvBD-12) to 47\%. Additional H3V8L21I29 for D3D8E21E29 substitutions further increased the hydrophobicity to $53 \%$ and the net positive charge from +1 (AvBD-12) to +5 (AvBD-12A1). Changes from $\mathrm{C}^{1} \mathrm{C}^{2} \mathrm{C}^{3} \mathrm{C}^{4} \mathrm{C}^{5} \mathrm{C}^{6}$ to S5S12S17A27A34A35 (AvBD-12A2) or to A5A12A17S27S34S35 (AvBD-12A3) and changes from D3D8E21E29 to R3K8K21R29 (AvBD-12A2 and A3) eliminated all three disulfide bonds and increased the peptide hydrophobicity from $33 \%$ to $40 \%$ and the net positive charge from +1 to +9 . AvBD-12A4, A5, and A6 respectively lost disulfide bond(s) $\mathrm{C}^{1-5} ; \mathrm{C}^{1-5}$ and $\mathrm{C}^{2-4} ; \mathrm{C}^{1-5}, \mathrm{C}^{2-4}$, and $\mathrm{C}^{3-6} \cdot \mathrm{C}$ to $\mathrm{U}$ changes did not affect the peptide hydrophobicity and charge. The hybrid AvBD-12/6 with the backbone of AvBD-12 and four amino acids (H3Q8Y21S29) of AvBD-6 retained parent peptide hydrophobicity (33\%) and an intermediate charge (+5) compared to AvBD-6 (+7) and AvBD-12 (+1). The 
sequence, charge, hydrophobicity, and numbers of disulfide bonds of analogs and wildtype AvBD-12 and AvBD-6 were listed in Table 3-1.

Superimposition of the predicted three-dimensional structures of AvBD-12 and the structure of human $\beta$-defensin 6 (hBD6) revealed a similar N-terminal $\alpha$-helix and an adjacent $\beta 2-\beta 3$ loop, in addition to the conserved internal $\beta$-sheet domains (Figure 3-1A). The $\alpha$-helix and $\beta 2-\beta 3$ loop have been identified by NMR spectroscopy as a contiguous binding surface for human CCR2 [151]. A comparison of the predicted three-dimensional structures of AvBD-6 and AvBD-12 showed only the $\beta 2-\beta 3$ loop in AvBD-6. AvBD-6 had an $\mathrm{N}$-terminal coil turn instead of an $\alpha$-helix. Peptide charge distribution analysis indicated that positively charged amino acid residues (H4R7R10R38R40) in the N- and C-termini of AvBD-6 formed a cluster whereas positively charged residues (H7R9K36) of AvBD-12 were separated by negatively charged residues (Figure 3-1D). The N-terminal $\alpha$-helix and the $\beta 2-\beta 3$ loop were also seen in the predicted structures of AvBD-12A2 and AvBD-12A3. However, differences in the $\beta 2-\beta 3$ loop between AvBD-12A2 and A3 were identified (Figure 3-2A). In AvBD-12A2, the $-\mathrm{C}=\mathrm{O}$ group in the main chain of $\mathrm{F} 28$ formed a hydrogen bond with the - $\mathrm{NH}$ group in the side chain of $\mathrm{R} 29$, resulting in the fold-back of R29 sidechain (Figure 3-2B). In AvBD-12A3, there was no hydrogen bond formation between R29 and F28. Instead, there are $\mathrm{C}-\mathrm{H} / \mathrm{O}$ interactions between the $-\mathrm{CH}$ groups in the aromatic $\pi$ ring of $\mathrm{F} 28$ and the $-\mathrm{C}=\mathrm{O}$ group of $\mathrm{F} 28$ as well as the $-\mathrm{CH}$ group in the side chain of S27, similar to what was reported previously [152]. Consequently, the side chain of R29 protruded towards the surface of the peptide and the aromatic ring of F28 in AvBD12A3 turned in parallel with R29 side chain (Figure 3-2C). 
Table 3-1. Amino acid sequences of avian $\beta$-defensin-12 (AvBD-12) analogs

\begin{tabular}{|c|c|c|c|c|}
\hline Defensin & Amino acid sequence ${ }^{a, b, c}$ & Charge & $\begin{array}{c}\text { \# of S-S } \\
\text { bonds }\end{array}$ & Hydrophobicity \\
\hline AvBD-12 & 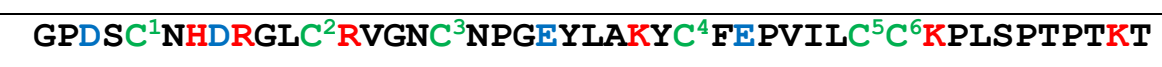 & +1 & 3 & $33 \%$ \\
\hline AvBD-12-A1 & GPHSANHVRGLARVGNANPGLYLAKYAFIPVILAAKPLSPTPTKT & +5 & 0 & $53 \%$ \\
\hline $\mathrm{AvBD}-12-\mathrm{A} 2$ & GPRSSNHKRGLSRVGNSNPGKYLAKYAFRPVILAAKPLSPTPTKT & +9 & 0 & $40 \%$ \\
\hline AvBD-12-A3 & GPRSANHKRGLARVGNANPGKYLAKYSFRPVILSSKPLSPTPTKT & +9 & 0 & $40 \%$ \\
\hline AvBD-12-A4 & GPDSUNHDRGLC ${ }^{2}$ RVGNC $^{3}$ NPGEYLAKYC ${ }^{4}$ FEPVILUC ${ }^{6} \mathrm{KPLSPTPTKT}^{2}$ & +1 & 2 & $33 \%$ \\
\hline AvBD-12-A5 & 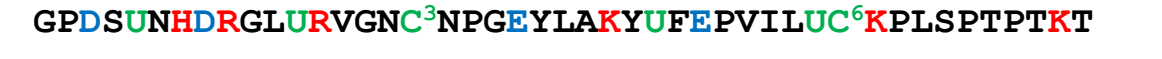 & +1 & 1 & $33 \%$ \\
\hline AvBD-12-A6 & GPDSUNHDRGLURVGNU NPGEYLAKYUFEPVILUUKPLSPTPTKT & +1 & 0 & $33 \%$ \\
\hline AvBD-12/6 & GPHSC ${ }^{1}$ NHQRGLC ${ }^{2}$ RVGNC $^{3}$ NPGYYLAKYC ${ }^{4}$ FSPVILC ${ }^{5} C^{6}$ KPLSPTPTKT & +5 & 3 & $33 \%$ \\
\hline AvBD-6 & SPIHAC ${ }^{1}$ RYQRGVC ${ }^{2}$ IPGPC ${ }^{3}$ RWPYYRVGSC ${ }^{4}$ GSGLKSC $C^{5} C^{6}$ VRNRWA & +7 & 3 & $33 \%$ \\
\hline
\end{tabular}

${ }^{a}$ Disulfide bonds (S-S) between $\mathrm{C}^{1-5}, \mathrm{C}^{2-4}, \mathrm{C}^{3-6}$. ${ }^{\mathrm{b}} \mathrm{U}$ : $\alpha$-aminobutyric acid. ${ }^{\mathrm{c}}$ Acidic amino acids, basic amino acids, and cysteines are highlighted in blue, red, and green color, respectively. $\mathrm{C}^{1} \mathrm{C}^{2} \mathrm{C}^{3} \mathrm{C}^{4} \mathrm{C}^{5} \mathrm{C}^{6}$ are $\mathrm{C} 5 \mathrm{C} 12 \mathrm{C} 17 \mathrm{C} 27 \mathrm{C} 34 \mathrm{C} 35$. 
A

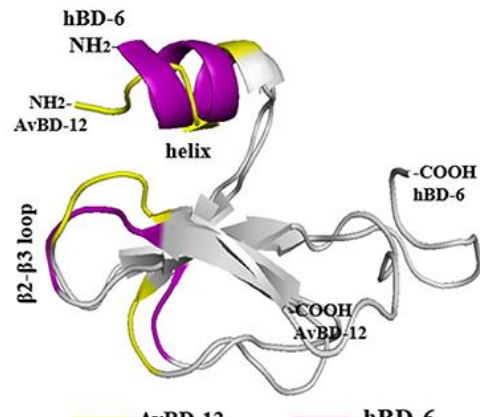

B

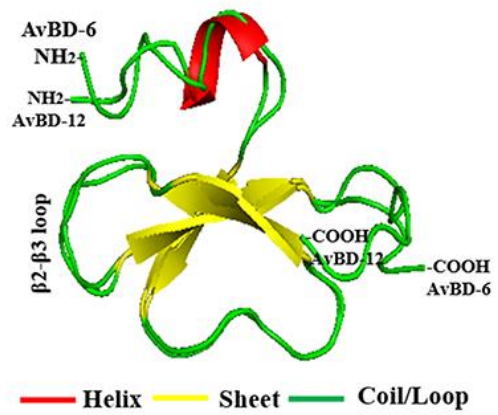

C

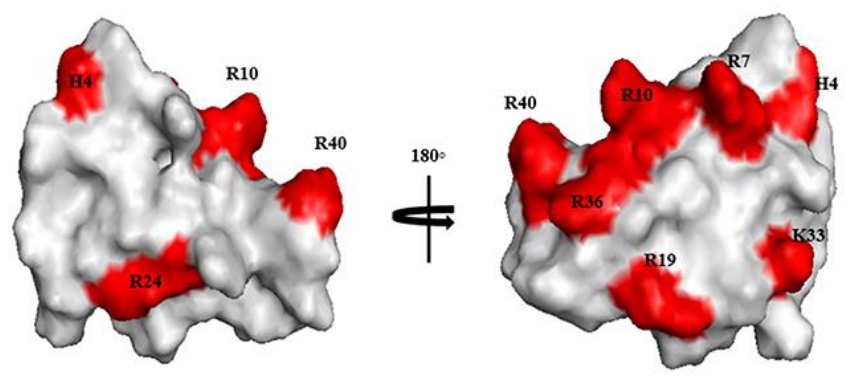

D
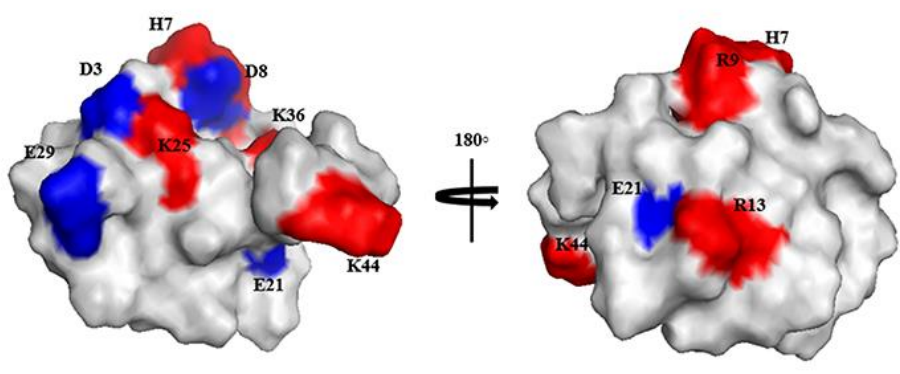

Figure 3-1.The predicted three-dimensional structures of AvBD-6 and AvBD-12

I-TASSER online service program was used to predict peptide structures.

Superimposition of the three-dimensional structures of AvBD-12 and hBD-6. The CCR2 binding surface of hBD6 is highlighted in purple and the corresponding region in AvBD12 is highlighted in yellow. (B) Superimposition of AvBD-12 and AvBD-6. (C) Distribution of positively charged amino acids in AvBD-6. (D) Distribution of positively and negatively charged amino acid residues in AvBD-12. Basic and acidic amino acids are highlighted in red and blue, respectively. 


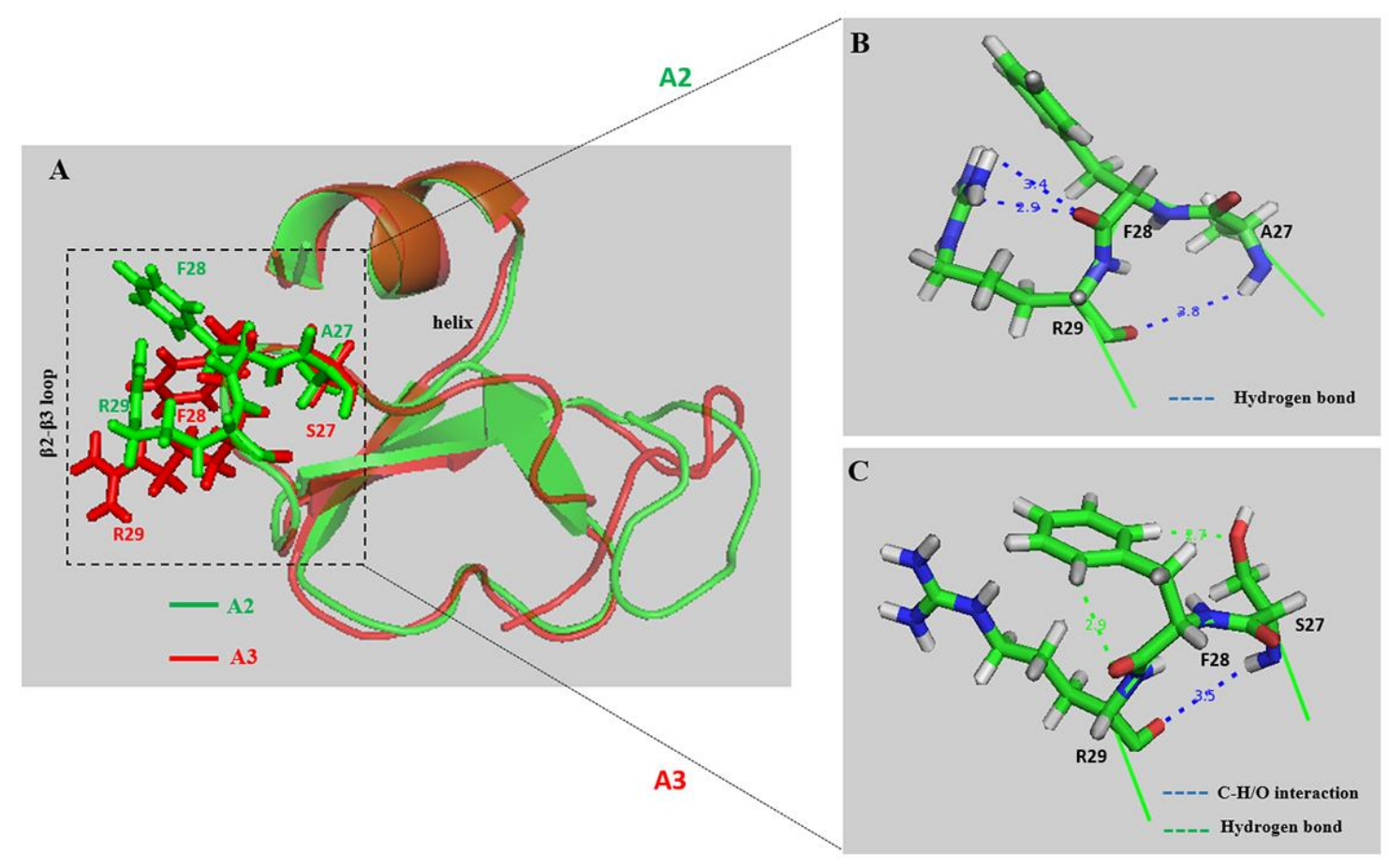

Figure 3-2. The predicted $\beta 2-\beta 3$ loop in AvBD-12A2 and AvBD-12A3

(A) Superimposition of AvBD-12A2 (green) and AvBD-12A3 (red) reveals the structural differences in the $\beta 2-\beta 3$ loop, a component of the CCR2 binding domain. (B) Enlarged review of the $\beta 2-\beta 3$ loop in $\mathrm{AvBD}-12 \mathrm{~A} 2$. The hydrogen bond between the $-\mathrm{C}=\mathrm{O}$ group of F28 main chain and the - $\mathrm{NH}$ group of R29 side chain causes the arginine residue to fold back. (C) Enlarged review of the $\beta 2-\beta 3$ loop in AvBD-12A3. The CHO interaction between $\mathrm{S} 27-\mathrm{OH}$ groups and the $-\mathrm{CH}$ groups on the aromatic ring of $\mathrm{F} 28$ results in an outward protrusion of R29 and a parallel twist of F28 aromatic ring. Distance: A. 


\section{Antimicrobial activity}

Group 1 analogs with increased net positive charge and hydrophobicity were significantly more effective $(\mathrm{p}<0.05)$ than the parent peptide AvBD-12 in killing E. coli, $S$. Typhimurium, P. aeruginosa, and $S$. aureus (Figure 3-3). At $16 \mu \mathrm{g} / \mathrm{ml}$, AvBD-12, AvBD12A1, AvBD-12A2, and AvBD-12A3 killed 74.4\%, 88.6\%, 100\%, 100\% of E. coli; 47.3\%, 95.6\%, 93.9\%, and 93.9\% S. Typhimurium; 35.5\%, 72.3\%, 98\%, and $100 \%$ of $P$. aeruginosa; and $52.9 \%, 77.2 \%, 83.1 \%$, and $91.7 \%$ of $S$. aureus, respectively. AvBD-12A2 and A3 with a net positive charge of +9 and hydrophobicity of $40 \%$ were more effective than AvBD-12A1 (charge $=+5$, hydrophobicity $=53 \%$ ). AvBD-12A2 and AvBD-12A3 which had identical charge and hydrophobicity but altered locations of alanine/serine residues exhibited different killing activities against E. coli or P. aeruginosa (Figure 3-3). The bactericidal potency of group-1 analogs can be ranked as AvBD-12A3 >AvBD12A2>AvBD-12A1>AvBD-12. Of the bacterial species tested, E. coli and P. aeruginosa were more susceptible than $S$. Typhimurium and $S$. aureus to AvBD-12A3 at medium concentrations, ranging from $8 \mu \mathrm{g} / \mathrm{ml}$ to $32 \mu \mathrm{g} / \mathrm{ml}$ (Figure $3-3$ ).

Group 2 analogs, including AvBD12A4, AvBD-12A5, and AvBD-12A6 showed similar killing activity to that of AvBD-12 (Figure 3-4). One exception was AvBD-12A4 was an exception that lost one disulfide bond $\left(\mathrm{C}^{1}-\mathrm{C}^{5}\right.$ or $\left.\mathrm{C} 5-\mathrm{C} 34\right)$ was shown to have weaker action than AvBD-12 against $S$. Typhimurium $(p<0.05)$. Group 3 or the hybrid analog, AvBD12/6 with the backbone of AvBD-12 and four amino acid residues of AvBD-6 exhibited similar killing activity to that of AvBD-6 (Figure 3-5). 
A
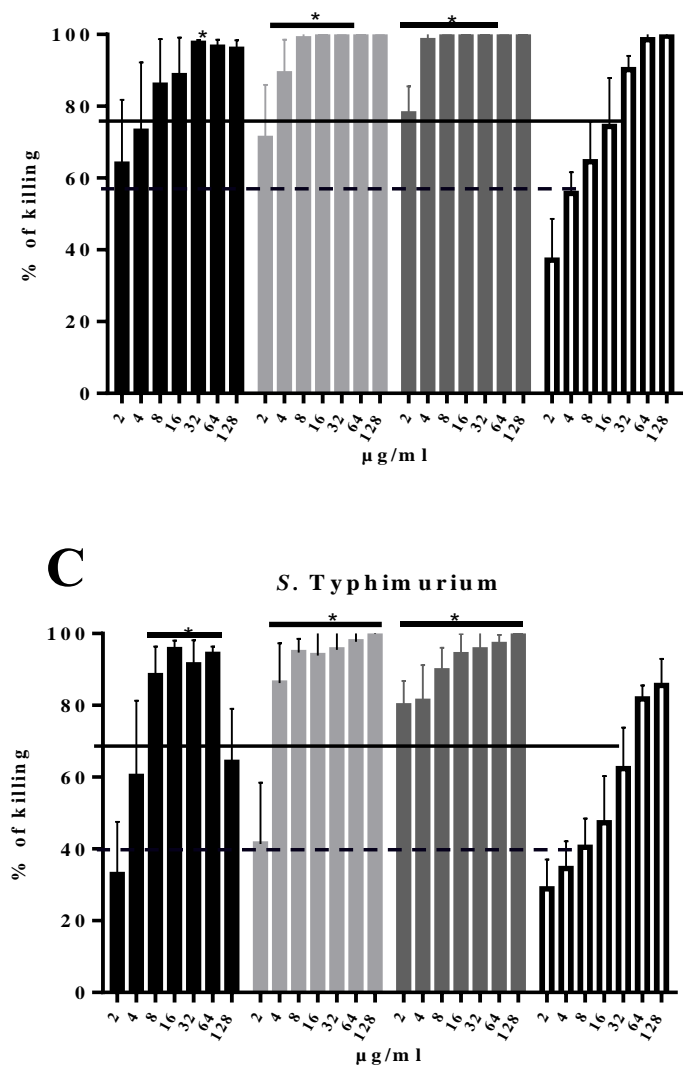
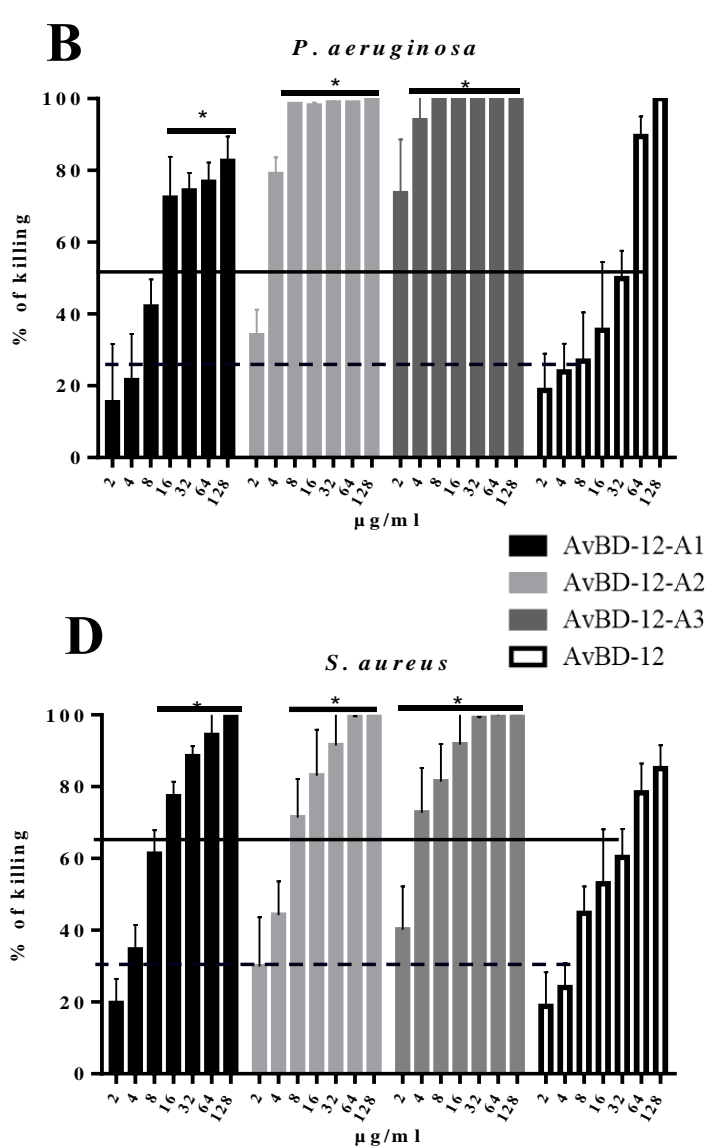

Figure 3-3. Antimicrobial activity of group 1 analogs

Bacteria $\left(10^{5} \mathrm{CFU} / \mathrm{ml}\right)$ were incubated with peptides at various concentrations, ranging from 2 to $128 \mu \mathrm{g} / \mathrm{ml}$ at $37{ }^{\circ} \mathrm{C}$ for $2 \mathrm{~h}$. Antimicrobial activity was presented as percent of killing compared to buffer-treated control. Antimicrobial activity of analogs against E. coli (A), P. aeruginosa (B), S. Typhimurium (C) and S. aureus (D). Wild-type AvBD-12 was included as the control. Data are means \pm SD $(n=3)$. Statistical analysis was performed using one-way analysis of variance (ANOVA) followed by Duncan's test for multiple comparisons using software SPSS version 19.0. Asterisk indicates the statistically significant difference between the analogs and AvBD-12 at the same concentrations $\left({ }^{*} p<\right.$ 0.05). Solid line: average killing percent of $32 \mu \mathrm{g} / \mathrm{ml}$ of wild-type AvBD-12. Dash line: average killing percent of $4 \mu \mathrm{g} / \mathrm{ml}$ of wild-type AvBD-12. 

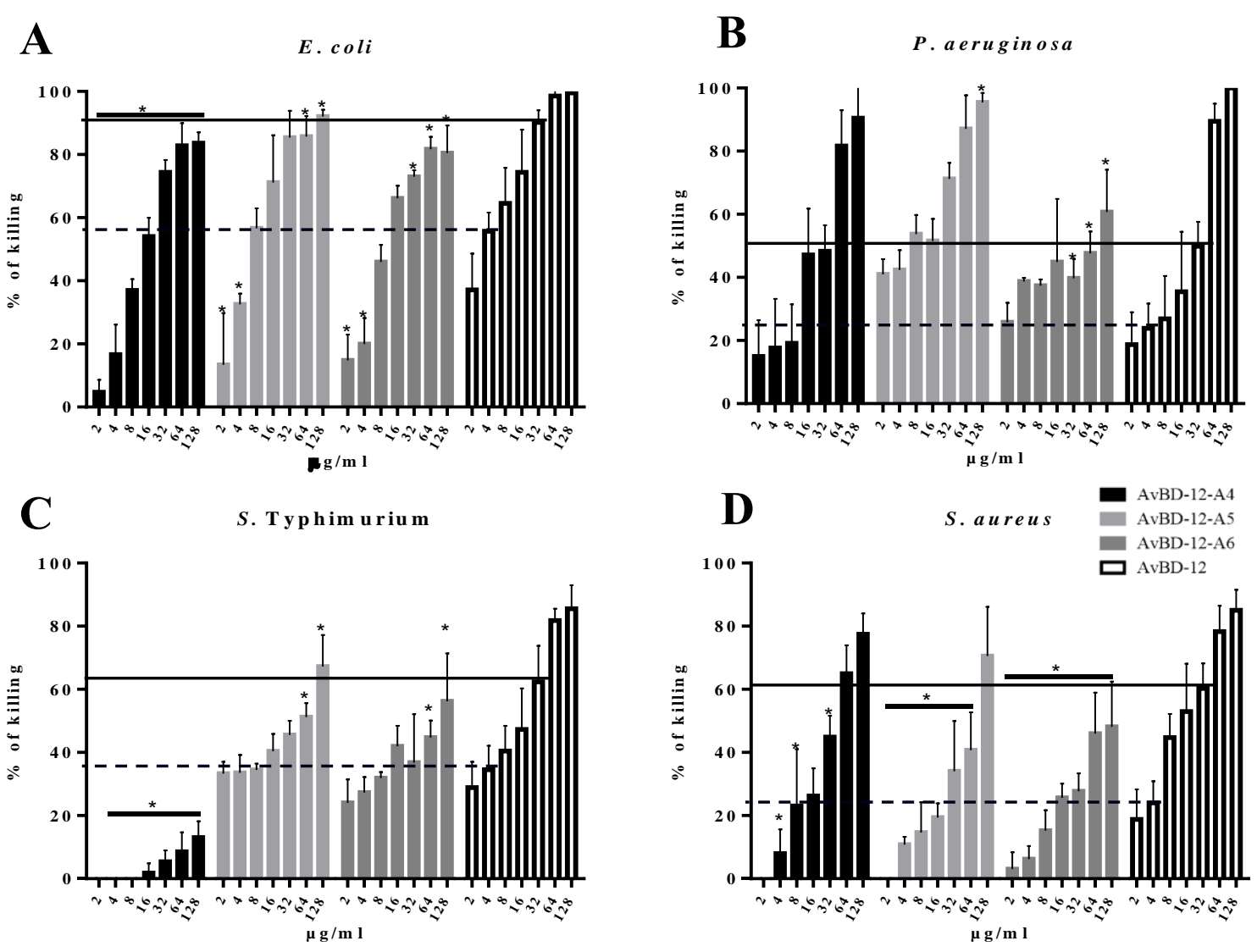

Figure 3-4. Antimicrobial activity of group 2 analogs

Bacteria $\left(10^{5} \mathrm{CFU} / \mathrm{ml}\right)$ were incubated with peptides at various concentrations, ranging from 2 to $128 \mu \mathrm{g} / \mathrm{ml}$, at $37^{\circ} \mathrm{C}$ for $2 \mathrm{~h}$. Antimicrobial activity was presented as percent of killing compared to buffer (non-AvBD)-treated control. Antimicrobial activity of analogs AvBD-12A4 to A6 against E. coli (A), P. aeruginosa (B), S. Typhimurium (C) and $S$. aureus (D). Wild-type AvBD-12 was included as the control. Data are means \pm SD (n=3). Asterisks indicate statistically significant difference between analogs and AvBD-12 at the same concentrations $\left({ }^{*} p<0.05\right)$. Solid line: average killing percent of $32 \mu \mathrm{g} / \mathrm{ml}$ of wildtype AvBD-12. Dash line: average killing percent of $4 \mu \mathrm{g} / \mathrm{ml}$ of wild-type AvBD-12. 
A

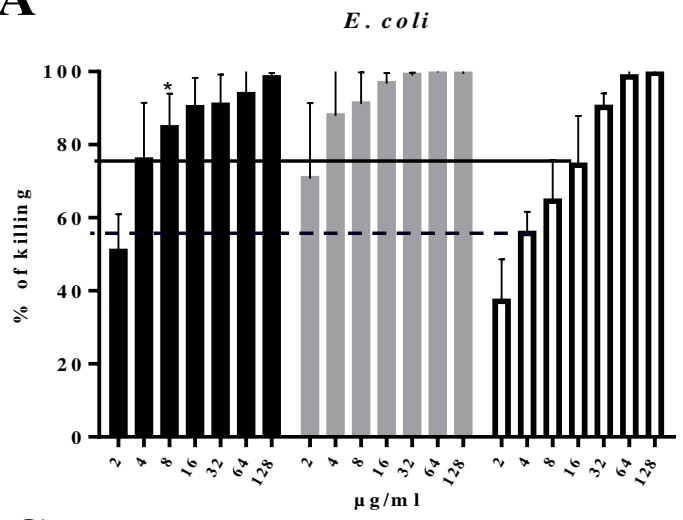

C

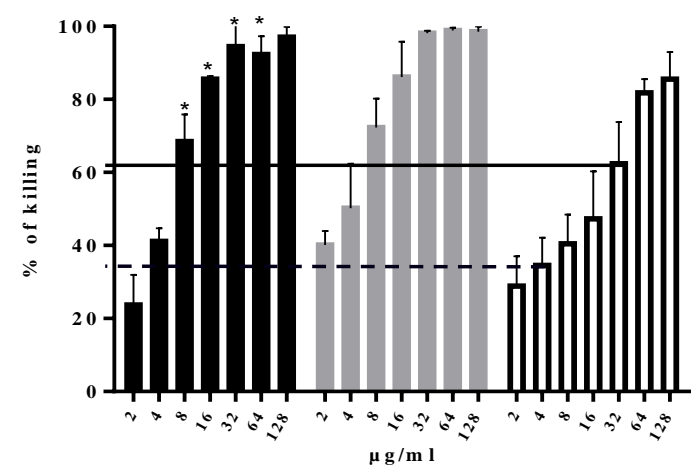

B
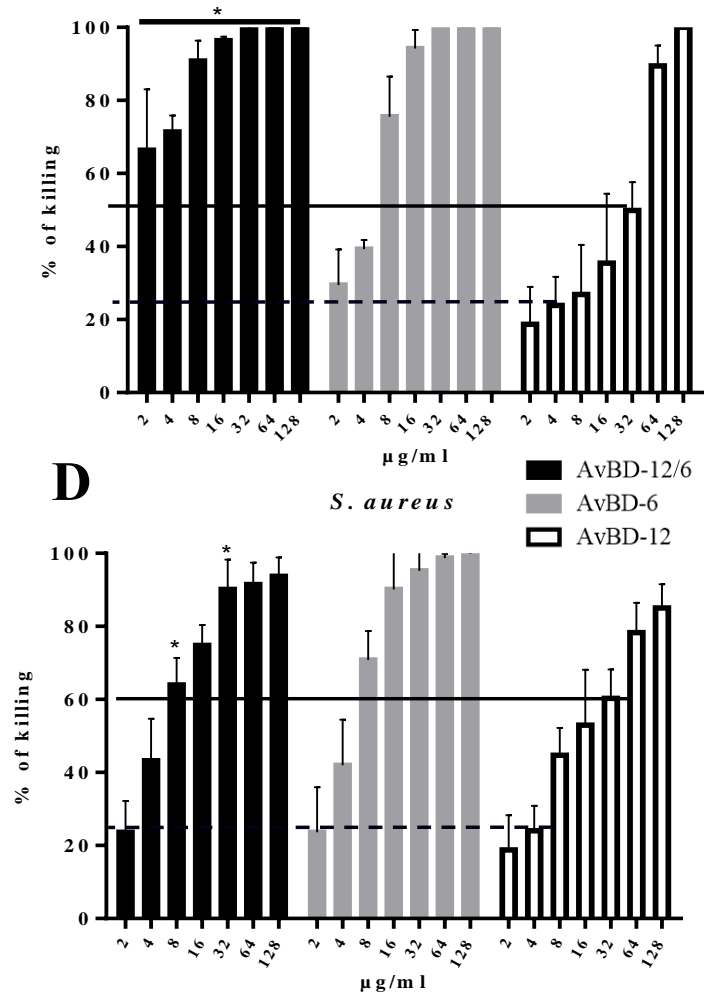

Figure 3-5. Antimicrobial activity of hybrid peptide AvBD-12/6

Bacteria $\left(10^{5} \mathrm{CFU} / \mathrm{ml}\right)$ were incubated with peptides at various concentrations, ranging from 2 to $128 \mu \mathrm{g} / \mathrm{ml}$, at $37^{\circ} \mathrm{C}$ for $2 \mathrm{~h}$. Antimicrobial activity was presented as percent of killing compared to buffer-treated control. Antimicrobial activity of analog AvBD-12/6 against E. coli (A), P. aeruginosa (B), S. Typhimurium (C) and S. aureus (D). Wild-type AvBD-6 and AvBD-12 were included as controls. Data are means \pm SD $(n=3)$. Asterisks indicate the statistically significant difference between the hybrid analog and AvBD-12 at the same concentrations $\left({ }^{*} p<0.05\right)$. Solid line: average killing percent of $32 \mu \mathrm{g} / \mathrm{ml}$ of wild-type AvBD-12. Dash line: average killing percent of $4 \mu \mathrm{g} / \mathrm{ml}$ of wild-type AvBD-12. 


\section{Minimum inhibitory concentration}

The MIC-ls of AvBD-12A1, AvBD-12A2 and AvBD-12A3 against E. coli, S. Typhimurium, and P. aeruginosa were 2 to 16-fold below that of AvBD-12, confirming the improved antimicrobial property of these analogs. The MIC-ls of these analogs against P. aeruginosa were 2 to 8-fold below that of AvBD-6. The ratio of MBC-1s/MIC-1s was equal to or below 4:1 for AvBD-12A2 and AvBD-12A3 against the three Gram-negative bacterial species tested, suggesting a bactericidal action [149]. The MIC-ls of AvBD-12A4, AvBD-12A5, and AvBD-12A6 with 2, 1 and 0 disulfide bonds, respectively, were higher than those of AvBD-12 and AvBD-6, indicating that removal of disulfide bonds compromised AvBD's antimicrobial function.

The MIC-ls of AvBD analogs was negatively correlated with the net positive charge. The correlation co-efficiencies (r) for E. coli, S. Typhimurium, P. aeruginosa, and S. aureus were $-0.7388, p<0.05 ;-0.8545, p<0.01 ;-0.8545, p<0.01$; and $-0.8727, p<0.01$, respectively. Although increasing hydrophobicity also resulted in improved antimicrobial potency, there was no a positive correlation found between hydrophobicity and antimicrobial activity.

Next, the MICs of AvBD-12A3, the most potent analog, hybrid analog AvBD-12/6, and the parent AvBD-6 and AvBD-12 were evaluated under conditions outlined in CLSI guidelines [117, 118]. High MICs (128 to $256 \mu \mathrm{g} / \mathrm{ml}$ or above) were obtained (Table 3-2). 
Table 3-2. The minimum inhibitory concentration and minimum bactericidal concentration of AvBD analogs

\begin{tabular}{|c|c|c|c|c|c|c|c|c|c|c|c|c|}
\hline \multirow{2}{*}{$\begin{array}{c}\text { Bacteria } \\
(\text { strain })\end{array}$} & \multicolumn{3}{|c|}{$\begin{array}{c}\text { E. coli } \\
\text { ( ATCC 25922) }\end{array}$} & \multicolumn{3}{|c|}{$\begin{array}{l}\text { S. Typhimurium } \\
\text { ( ATCC 14028) }\end{array}$} & \multicolumn{3}{|c|}{$\begin{array}{c}\text { P. aeruginosa } \\
\text { (ATCC 27853) }\end{array}$} & \multicolumn{3}{|c|}{$\begin{array}{c}\text { S. aureus } \\
\text { (ATCC 29213) }\end{array}$} \\
\hline & MIC-ls & MBC-ls & MIC & MIC-ls & MBC-1s & MIC & MIC-ls & MBC-ls & MIC & MIC-ls & MBC-ls & MIC \\
\hline AvBD-12-A1 & 8 & 32 & N/A & 32 & 128 & N/A & 32 & 256 & N/A & 256 & $>256$ & N/A \\
\hline $\mathrm{AvBD}-12-\mathrm{A} 2$ & 4 & 16 & N/A & 16 & 64 & N/A & 16 & 32 & N/A & 256 & 256 & N/A \\
\hline AvBD-12-A3 & 4 & 16 & 128 & 16 & 64 & $>256$ & 8 & 16 & $>256$ & 128 & 256 & $>256$ \\
\hline AvBD-12-A4 & 128 & $>256$ & N/A & 256 & $>256$ & N/A & 256 & $>256$ & N/A & $>256$ & $>256$ & N/A \\
\hline $\mathrm{AvBD}-12-\mathrm{A} 5$ & 64 & 256 & N/A & 256 & $>256$ & N/A & 256 & $>256$ & N/A & $>256$ & $>256$ & N/A \\
\hline $\mathrm{AvBD}-12-\mathrm{A} 6$ & 64 & 256 & N/A & 256 & $>256$ & N/A & 256 & $>256$ & N/A & $>256$ & $>256$ & N/A \\
\hline AvBD-12/6 & 8 & 32 & 256 & 16 & 64 & $>256$ & 64 & 256 & $>256$ & 256 & 256 & 256 \\
\hline AvBD-6 & 4 & 16 & 128 & 16 & 64 & $>256$ & 64 & 128 & $>256$ & 256 & 256 & 256 \\
\hline AvBD-12 & 32 & 128 & 256 & 128 & 256 & $>256$ & 128 & $>256$ & $>256$ & 256 & 256 & $>256$ \\
\hline
\end{tabular}




\section{Susceptibility to $\mathrm{NaCl}$}

The impact of $\mathrm{NaCl}$ on the killing activity of $\mathrm{AvBDs}$ was assessed at $\mathrm{NaCl}$ concentrations ranging from 5 to $150 \mathrm{mM}$. Increasing $\mathrm{NaCl}$ concentration had a less adverse impact on the killing activity of AvBD-12A2 and AvBD-12A3 which had a higher net positive charge (+9) and hydrophobicity (40\%) than AvBD-12 (Figure 3-6). AvBD-12A1, the most hydrophobic (53\%) analog, showed similar or increased susceptibility to $\mathrm{NaCl}$, compared to AvBD-12 (Figure 3-6). Susceptibility to $\mathrm{NaCl}$ was also influenced by the bacterial species under investigation. At $150 \mathrm{mM} \mathrm{NaCl}, \mathrm{AvBD}-12 \mathrm{~A} 2$ and AvBD-12A3 retained approximately $80 \%$ of killing potency against $E$. coli and $P$. aeruginosa, but only $30 \%$ to $60 \%$ of killing activity against $S$. Typhimurium and S. aureus (Figure 3-6). AvBD-12A4, AvBD-12A5, and AvBD-12A6 with fewer disulfide bonds were equally or more susceptible to $\mathrm{NaCl}$ than AvBD-12 (Figure 3-7). The effect of $\mathrm{NaCl}$ on the antimicrobial activity of AvBD-12/6 with the backbone of AvBD-12 and increased net positive charge (+5) resembled AvBD-6 instead of AvBD-12 (Figure 3-8). 
A

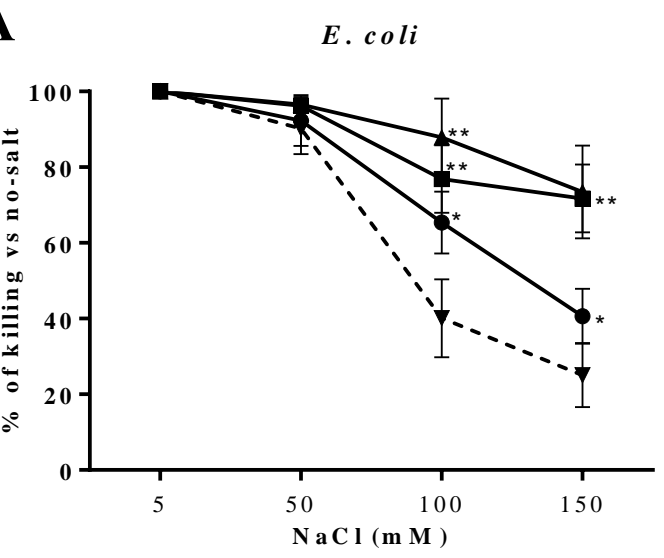

C

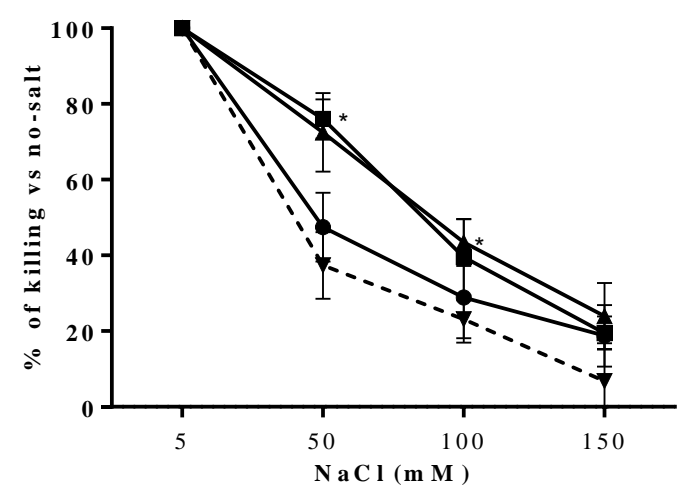

B

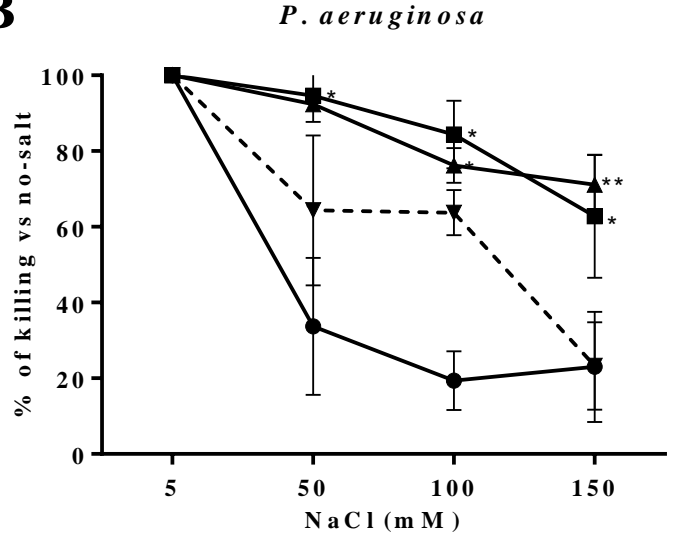

D
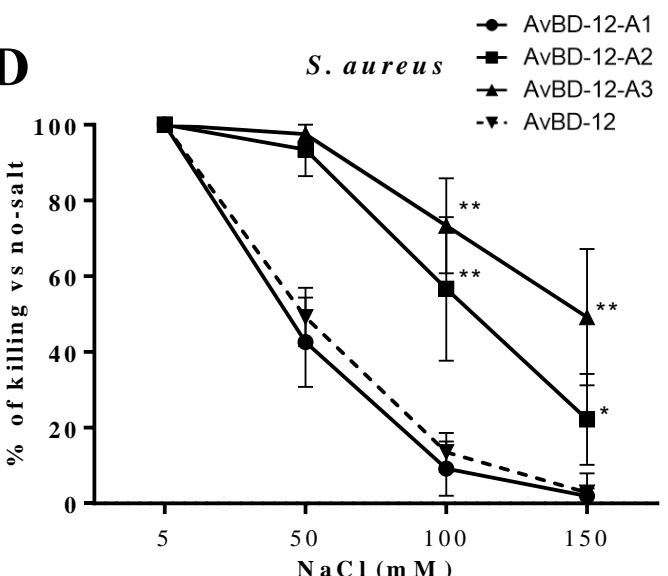

Figure 3-6. Effect of $\mathrm{NaCl}$ on the antimicrobial activity of group one AvBDs

Bacteria were treated with group one analogs in the presence of $5 \mathrm{mM}, 50 \mathrm{mM}, 100 \mathrm{mM}$ or $150 \mathrm{mM} \mathrm{NaCl}$. AvBDs were used at the following concentrations: $16 \mu \mathrm{g} / \mathrm{ml}$ against $E$. coli (A), $32 \mu \mathrm{g} / \mathrm{ml}$ against P. aeruginosa (B) and $S$. Typhimurium (C) and $64 \mu \mathrm{g} / \mathrm{ml}$ against S. aureus (D). These concentrations were chosen to ensure more than $50 \%$ of the killing of inoculum by the majority of analogs. Results are expressed as percent of killing compared to the no-salt control. Data shown are means $\pm \mathrm{SD}(\mathrm{n}=3)$. Statistical analysis was performed using the one-way analysis of variance (ANOVA) followed by Duncan's test for multiple comparisons using software SPSS version 19.0 (IBM Corp., Armonk, NY). Asterisks indicate statistically significant difference among different treatment groups $\left({ }^{*} p<0.05\right.$, $* * p<0.01)$. 
A

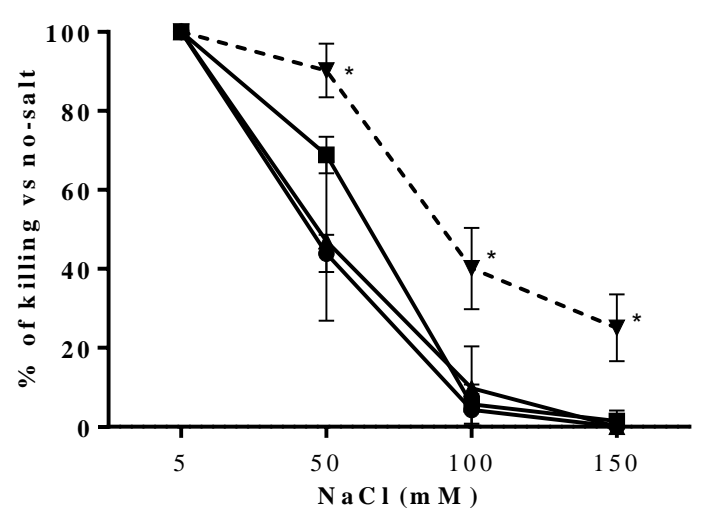

$\mathbf{C}$

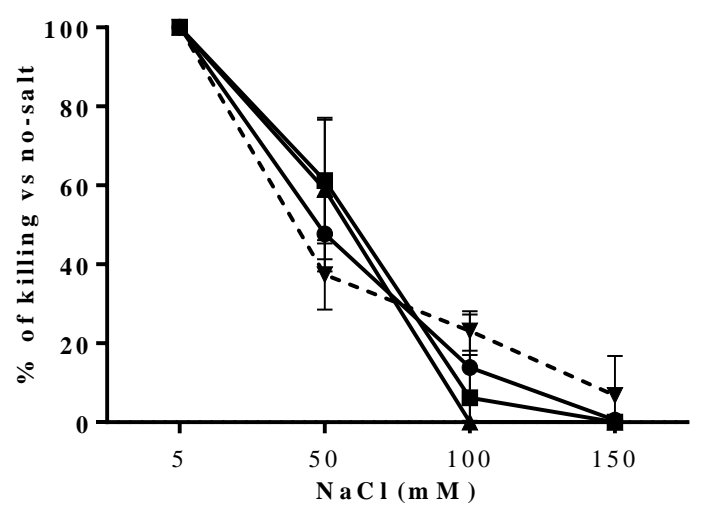

B

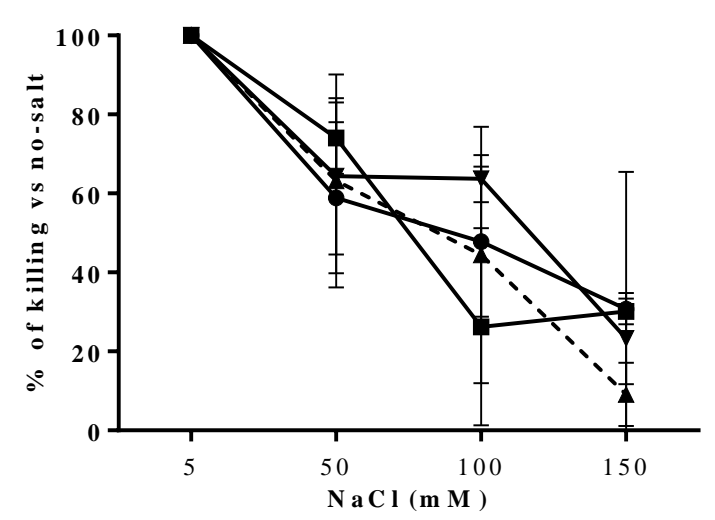

D

S. aureus

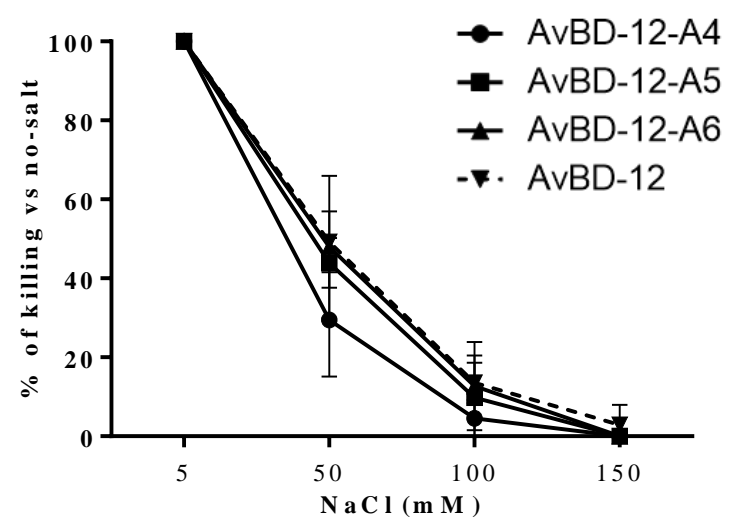

Figure 3-7. Effect of $\mathrm{NaCl}$ on the antimicrobial activity of group 2 analogs

Bacteria were treated with group two analogs in the presence of $5 \mathrm{mM}, 50 \mathrm{mM}, 100 \mathrm{mM}$ or $150 \mathrm{mM} \mathrm{NaCl}$. Results are expressed as percent of killing compared to the no-salt control. Data shown are means \pm SD $(n=3)$. Statistical analysis was performed using the one-way analysis of variance (ANOVA) followed by Duncan's test for multiple comparisons using software SPSS version 19.0 (IBM Corp., Armonk, NY). Asterisks indicate statistically significant difference among different treatment groups $\left({ }^{*} p<0.05,{ }^{*} p<0.01\right)$. 

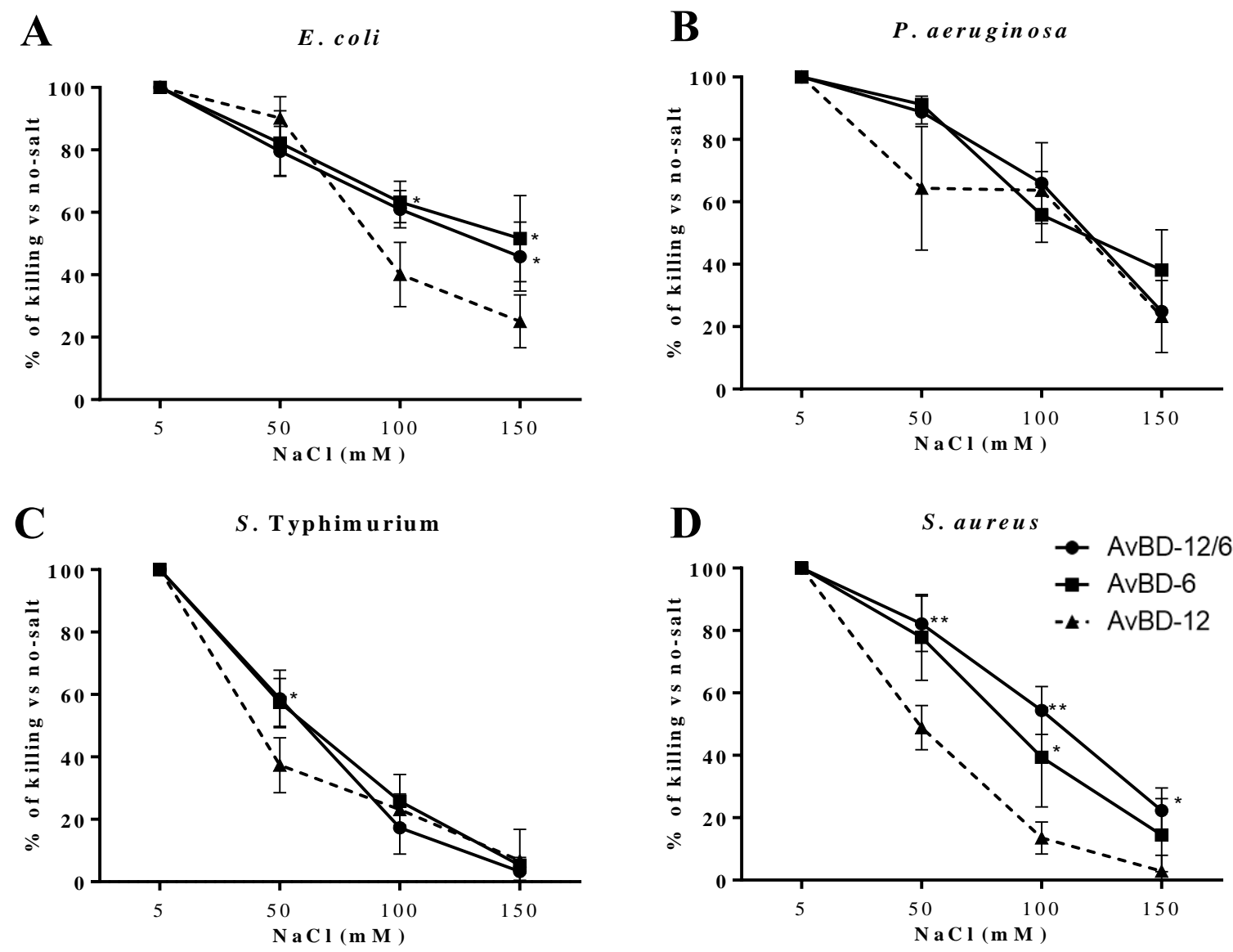

Figure 3-8. Effect of $\mathrm{NaCl}$ on the antimicrobial activity of analog AvBD-12/6

Bacteria were treated with AvBDs in the presence of $5 \mathrm{mM}, 50 \mathrm{mM}, 100 \mathrm{mM}$ or $150 \mathrm{mM}$ $\mathrm{NaCl}$. Data shown are means $\pm \mathrm{SD}(\mathrm{n}=3)$. Statistical analysis was performed using the oneway analysis of variance (ANOVA) followed by Duncan's test for multiple comparisons using software SPSS version 19.0 (IBM Corp., Armonk, NY). Asterisks indicate statistically significant difference among different treatment groups $\left({ }^{*} p<0.05,{ }^{* *} p<0.01\right)$. 


\section{Cytotoxicity to host cells}

Cell cytotoxicity of most potent analog AvBD-12A3 (+9) and the hybrid analog AvBD12/6 (+5) was determined. Exposure of chicken macrophage cell lines HD11 and MQNCSU, murine dendritic cell line JAWSII and CHO-K1 cells to AvBDs at concentrations of $4,16,64,256 \mu \mathrm{g} / \mathrm{ml}$ for $4,12,24$, and $48 \mathrm{~h}$ did not affect cell variability (Data was not shown). The results were consistent with our previous findings that AvBD-6 and AvBD12 were non-cytotoxic to avian and mammalian cell lines [59].

\section{Chemotactic activity}

Group 1 analogs AvBD-12A1, AvBD-12A2, and AvBD-12A3 showed minimal chemotactic activity for CCR2-CHO cells (Figure 3-9A). However, AvBD-12A2 and AvBD-12A3 at $64 \mu \mathrm{g} / \mathrm{ml}$ demonstrated mild (C.I. $=2.37 ; 35.9 \%$ of wild-type) and modest (C.I. $=3.74 ; 56.6 \%$ of wild-type) chemotactic activity for JAWSII cells (Figure 3-9B), respectively. Analogs AvBD-12A4 with two disulfide bonds had a mild chemotactic activity for CCR2-CHO (C.I. $=1.48$ to 2.18$)$ and JAWSII cells $($ C.I. $=1.05$ to 1.86$)$ which were significantly below the chemotactic index of parent peptide AvBD-12 $(p<0.01$, Figure 3-9C and 9D). AvBD-12A5 with one disulfide bond and AvBD-12A6 without disulfide bond lost their chemotactic activity for both CCR2-CHO-K1 and JAWSII cells (Figure 3-9C and 9D). The hybrid AvBD-12/6 retained the chemotactic function of the backbone peptide AvBD-12 (Figure 3-9E and 9F). 
$\mathbf{A}$

CCR2 tranfected CHO-K1

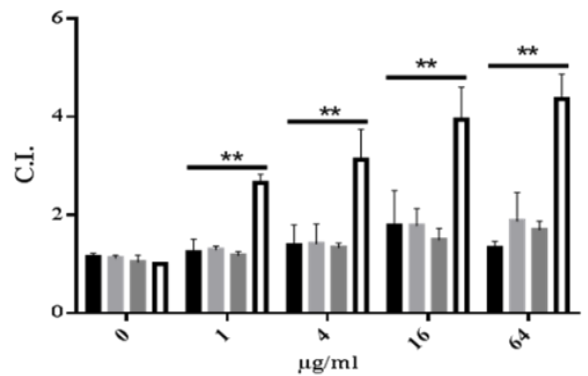

C

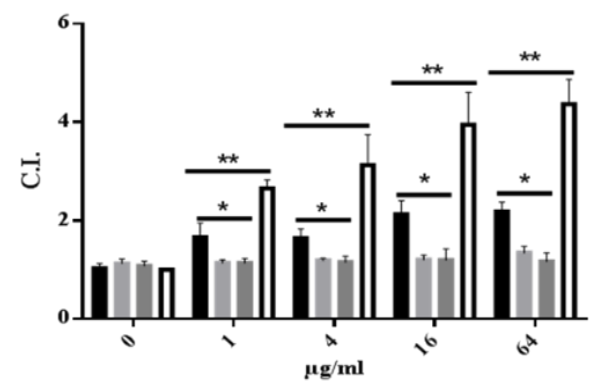

$\mathbf{E}$

CCR2 tranfected CHO-K1

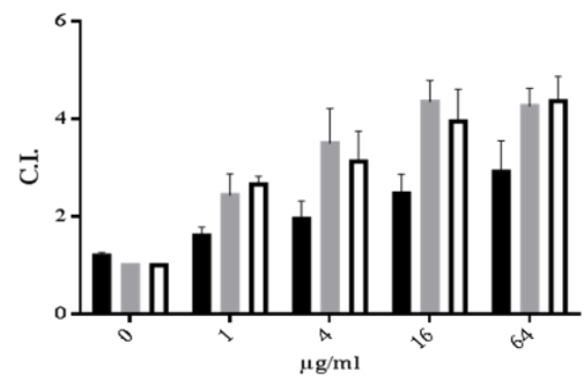

B

JAWSI

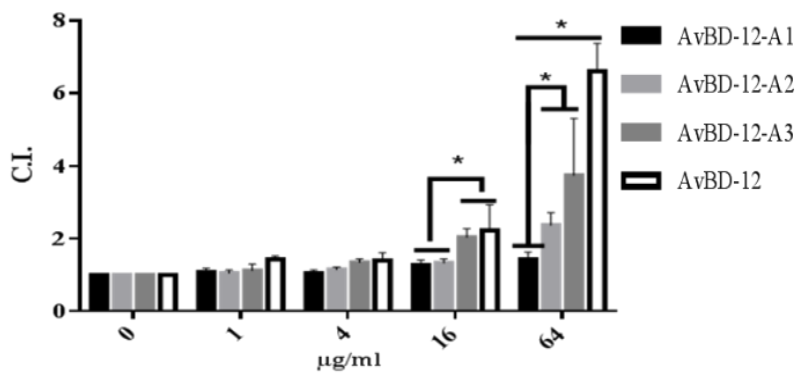

D

JAWSII

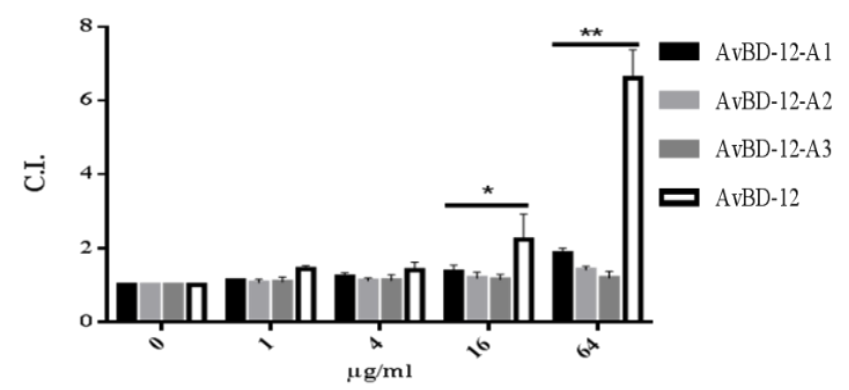

$\mathbf{F}$

JAWSII

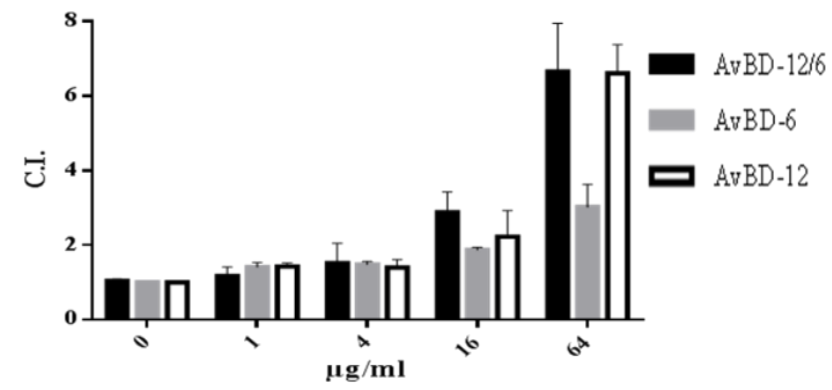

Figure 3-9. Chemotactic activity of AvBD-12 analogs for CCR2 transfected CHO-K1 cells and mouse immature dendritic JAWSII cells

Migration of CCR2 transfected $\mathrm{CHO}-\mathrm{K} 1$ cells (A to $\mathrm{C}$ ) and mouse immature dendritic JAWSII cells (D to F). The results are expressed as chemotactic indexes (C.I.): the number of migrated cells induced by AvBD analogs / the number of migrated cells in response to chemotactic buffer. Data are means $\pm \mathrm{SD}(\mathrm{n}=5)$. Asterisks indicate statistically significant difference between analogs and wild-type AvBD-12 $(* p<0.05, * * p<0.01)$. 


\section{SEM observation}

Mid-logarithmic-phase $S$. Typhimurium bacteria treated with AvBD-12A3 (Figure 3-10A), AvBD-12/6 (Figure 3-10B), AvBD-12 (Figure 3-10C) and AvBD-6 (Figure 3-10D) displayed cell membrane damage (arrow 1) and cell deformation (arrow 2). Midlogarithmic-phase $S$. Typhimurium cells treated with PBS showed normal size and intact structure (Figure 3-10F, arrow 4). Cell death in stationary-phase culture (Figure 3-10E, arrow 3) showed loss of intracellular content and uniform membrane structure. 

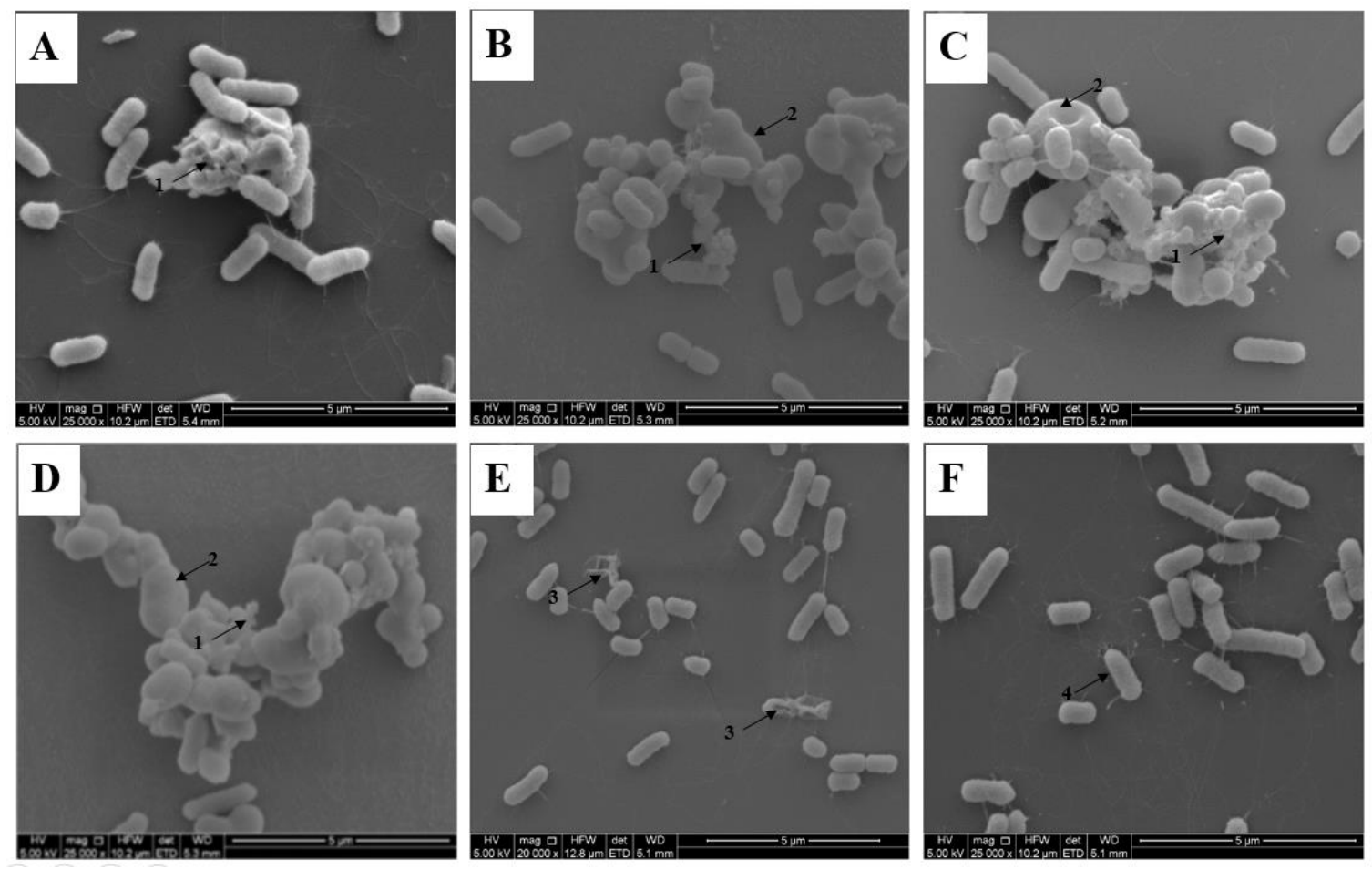

Figure 3-10. Scanning electron microscopy of $S$. Typhimurium treated with AvBDs

Mid-logarithmic-phase $S$. Typhimurium cells $\left(10^{8} \mathrm{CFU}\right)$ were incubated with AvBDs at a final concentration of $1 \times$ MIC-1s for 30 min. (A) AvBD-12A3, (B) AvBD-12/6, (C) AvBD-12, (D) AvBD-6, (E) Stationary phase bacteria, (F) Mid-logarithmic-phase $S$. Typhimurium treated with PBS. Arrow 1, membrane damage. Arrow 2, cell deformation. Arrow 3, cell death in stationary-phase culture. Arrow 4, a normal cell in mid-logarithmicphase culture. Scale bar: $5 \mu \mathrm{m}$. 


\section{Discussion}

Due to the broad spectrum antimicrobial activity, LPS-neutralizing property, immunomodulatory function and the low cell cytotoxicity, defensins may serve as natural antimicrobial peptides or templates for novel drug design $[9,52,59,148,153]$. It has been reported that the three conserved disulfide bonds are required for the chemotactic function, but not the antimicrobial activity of mammalian $\beta$-defensins [64, 121-124]. However, data from our previous studies indicate that the correct formation of disulfide bonds via oxidative folding is required for maximum antimicrobial activity $[57,59]$. In an effort to identify key structural components important to the antimicrobial and chemotactic activities of AvBDs, we compared the predicted three-dimensional structures of AvBDs with the well-characterized structure of human beta-defensin 6 (hBD6). It was previously shown that the $\mathrm{N}$-terminal $\alpha$-helix and an adjacent $\beta 2-\beta 3$ loop form a contiguous binding surface for CCR2 [151]. The presence of a similar CCR2 binding domain in AvBD-12 may account for the chemotactic activity of AvBD-12 for both avian and mammalian immune cells because AvBD-6 which had an N-terminal coil instead of the $\alpha$-helix was chemotactic only for avian cells [59].

To further understand the structure-activity relationship, we designed and evaluated seven synthetic analogs of AvBD-12. In the first group of analogs, disulfide bonds were removed by replacing cysteines with structurally similar residues (alanine or serine) whereas peptide hydrophobicity and charge were increased by substituting negatively charged residues with hydrophobic (AvBD-12A1) or positively charged residues (AvBD-12A2 and A3). All members of the first group demonstrated stronger antimicrobial activity than their parent peptide AvBD-12, indicating that cysteine-free (linear) AvBDs with a high net positive 
charge and moderate hydrophobicity can be potent antimicrobial agents. Such linear peptides can be synthesized without oxidative folding which simplifies the production process and reduces cost. In the present study, anti-S. Typhimurium activity of AvBD12A1 decreased significantly as peptide concentration increased from $64 \mu \mathrm{g} / \mathrm{ml}$ to 128 $\mu \mathrm{g} / \mathrm{ml}$ (Figure 3-3C). Similar results were obtained repeatedly, suggesting that the decrease was unlikely caused by technical errors. We hypothesize that AvBD-12A1, the most hydrophobic (53\%) peptide may form aggregates which interfere with peptide binding to Salmonella membrane components, resulting in a reduction in antimicrobial potency. Interestingly, AvBD-12A2 and AvBD-12A3 with identical charge and hydrophobicity, but different locations of three alanine and three serine residues (AvBD-12A3: S5S12S17A27A34A35 and AvBD-12A3: A5A12A17S27S34S35) showed a significant difference in antimicrobial and chemotactic activities. Structural analysis of AvBD-12A3 revealed an outward protrusion of R29 side chain and parallel twist F28 aromatic ring. We hypothesize that the outward protrusion of a positively charged residue and an adjacent aromatic ring enhances the interaction between AvBD-12A3 and microbial surface components or CCR2, thereby facilitating antimicrobial and chemotactic functions.

To assess the role of disulfide bonds independent of charge and hydrophobicity, we replaced cysteine residues with isosteric $\alpha$-aminobutyric acids (Abu, $\mathrm{U}$ ) to create group 2 analogs. Our data indicated that missing even one conserved disulfide bridge resulted in a significant reduction in AvBD's chemotactic activity. Elimination of two or three disulfide bonds completely abolished the chemotactic activity for both avian CCR2-positive cells and murine immature dendritic cells. It has been shown that $\mathrm{C}^{1}$ and $\mathrm{C}^{4}$ essential to the formation of first and second disulfide bonds are located at the CCR2 binding center [151]. 
Thus substitution of $\mathrm{C}$ with $\mathrm{U}$ may have disrupted the CCR2 binding surface, resulting in the loss of chemotactic activity. Our data also showed that removal of disulfide bonds had varying degrees of negative impact on AvBD's antimicrobial activity. Analog AvBD-12A4 missing the $\mathrm{C}^{1}-\mathrm{C}^{5}$ bond was nearly inactive against $S$. Typhimurium. These results collectively suggest that all three disulfide bonds are needed in the natural form of AvBDs to maintain tertiary structural features that are critical to interact with microbial surface components and CCRs on immune cells.

Besides antimicrobial assays, SEM was performed to illustrate the killing mechanisms of AvBD-12 analogs. Treatment of $S$. Typhimurium with AvBD-12A3 and AvBD-12/6, and wild-type AvBD-6 and AvBD-12 caused similar ultrastructural changes, including cell deformation and membrane damage. Giant cells were only observed among treatment groups, indicating membrane permeabilization is an essential step in the killing of microbes by AvBDs.

Salt sensitivity is a major obstacle to the application of $\beta$-defensins as chemotherapeutic agents. Different strategies have been explored to increase the resistance of beta-defensins to salts, such as N-terminal deletion [79], combining sequences of HBD-1 and salt-resistant $\theta$-defensin [93] and replacement of tryptophan or histidine with a bulky amino acid $\beta$ naphthylalanine [76]. In the present study, we found that increasing peptide charge significantly reduced the impact of $\mathrm{NaCl}$ on the antimicrobial efficacy of AvBD-12A2 and AvBD-12A3. However, the activity against S. Typhimurium was still severely inhibited by the physiological concentration of $\mathrm{NaCl}(150 \mathrm{mM})$, suggesting that different mechanisms are involved in killing different bacterial species.

In the present study, we also determined the minimum inhibitory concentrations (MICs) of 
AvBDs. Using the standard Muller Hinton II broth, the MICs of the parent AvBDs and the most potent analog AvBD-12A3 were much higher than the MIC values obtained under the low-salt and low-nutrient condition. We hypothesize that interference of initial peptidebacteria interactions by cationic salts as well as enzymatic degradation or modification of AvBDs during bacterial growth in a nutrient-rich Mueller-Hinton broth might have contributed to the decreased antimicrobial activity. It has been shown that human cathelicidin LL-37 could be hydrolyzed by $S$. aureus protease or cleaved by metalloprotease, gelatinase or cysteine protease produced by other bacterial species [154]. Proteolytic degradation is one of the main mechanisms that both Gram-positive and Gramnegative bacteria use to evade host antimicrobial peptide killing [155-160]. A further modification is clearly needed to improve the efficacy of AvBD-12A3 as an antimicrobial agent. It is noteworthy that all AvBD analogs remain non-cytotoxic to avian and mammalian cells.

\section{Conclusions}

The three conserved disulfide bonds maintaining the tertiary structure of natural AvBDs are required not only for the chemotactic activity but also for maximum antimicrobial activity. AvBD-12A3 with increased net positive charge and a CCR2-binding domain (Nterminal $\alpha$-helix and $\beta 2-\beta 3$ loop) demonstrated potent antimicrobial activity and retained the partial chemotactic property. Analog AvBD-12A3 may serve as a template for the design of novel antimicrobial peptides as therapeutic agents for both avian and mammalian hosts. 


\title{
CHAPTER 4: Beta-Defensin Derived Cationic Antimicrobial Peptides with Potent Killing Activity against Gram-Negative and Gram-Positive \\ Bacteria
}

Revised manuscript, BMC Microbiology 2018

\begin{abstract}
Avian $\beta$-defensins (AvBD) are cationic antimicrobial peptides (CAMP) with broadspectrum antimicrobial activity, chemotactic property, and low host cytotoxicity. However, their bactericidal activity is greatly compromised under physiological salt concentrations which limits the use of these peptides as antimicrobial therapeutic agents. The length and the complex structure involving three conserved disulfide bonds are additional drawbacks associated with high production cost. In the present study, an integrated design approach was adopted to develop four short CAMPs (11 to 25 a.a. residues) based on the key functional components of AvBDs and their biological functions were evaluated. CAMP-t1 contained the CCR2 binding domain (N-terminal loop and adjacent $\alpha$-helix) of AvBD-12 whereas CAMP-t2 was comprised of the key a.a. residues contributing to the concentrated positive surface charge and hydrophobicity of AvBD-6. Both CAMP-t1 and CAMP-t2 demonstrated strong antimicrobial activity against Pseudomonas aeruginosa, Staphylococcus aureus and Staphylococcus pseudintermedius. However, CAMP-t1 failed to show chemotactic activity and CAMP-t2, although superior in killing Staphylococcus spp., remained sensitive to salts. CAMP-t2 was further modified to yield two $\alpha$-helical peptides CAMP-A and CAMP-B by eliminating potential protease cutting sites, incorporating a $\mathrm{C}$-terminal poly-Trp tail, $\mathrm{N}$-terminal acetylation, and $\mathrm{C}$-terminal amidation. CAMP-A and CAMP-B demonstrated significantly improved antimicrobial activity
\end{abstract}


against multidrug-resistant $P$. aeruginosa and methicillin-resistant $S$. pseudintermedius strains (MRSP) and following advantageous characteristics: rapid membrane attacking (30 $\mathrm{min}$ ), resistance to physiological concentrations of $\mathrm{NaCl}$ and $\mathrm{CaCl}_{2}$, resistance or tolerance to protease digestion. CAMP-A and CAMP-B showed a therapeutic index of 4.5 or greater, suggesting potentially safe for clinical use. In conclusion, CAMP-A and CAMP-B designed with an integrated strategy have applicable features including strong antimicrobial activity, resistance to salts and proteases, low cytotoxicity, rapid membrane attacking, and moderate therapeutic index, which collectively suggests that they are excellent candidates for the treatment of multidrug-resistant $P$. aeruginos $a$ and MRSP.

\section{Background}

The rapid emergence and spread of antimicrobial resistance, particularly those associated with Pseudomonas aeruginosa and Staphylococcus spp., have become a serious threat to public health $[3,143]$. The CDC estimated that each year in the United States there were approximately 88,000 cases and 11,000 deaths due to infections with methicillin-resistant Staphylococcus aureus (MRSA) [161]. Various studies have been conducted to search for new classes of antimicrobial therapeutic agents or antibiotic alternatives with novel targets and modes of action [162]. Host cationic antimicrobial peptides (CAMPs), including linear peptides, $\alpha$-helical peptides, circular and complex structures with loops and $\beta$-sheets constitute the first line of innate defense against microbial pathogens [163]. The features shared by these CAMPs are net positive charge and amphipathicity [25]. The cationic property of CAMPs allows for the initial interaction of the peptide with the anionic surface groups of the microbial membrane and the hydrophobicity enables the peptide to integrate into the hydrophobic core of microbial membrane. The mechanisms of action of CAMPs 
are complicated and achieved primarily through membrane damage and possibly subsequent interactions with cellular molecules, which make the potential development of microbial resistance less likely [25]. A major group of CAMPs with broad-spectrum antimicrobial activity is $\beta$-defensins which contain three cysteine-cysteine disulfide bonds [11]. In addition to their antimicrobial activity and low potential for the development of resistance by bacteria, $\beta$-defensins have several other beneficial features, such as modulating host immune response (e.g. chemoattracting immune cells) [12, 17, 51, 164]. Our previous studies show that avian $\beta$-defensins (AvBDs) such as AvBD-6 and AvBD12 possess the following biological properties: broad-spectrum antimicrobial activity, LPSneutralizing ability, chemotactic activity, and minimal cell cytotoxicity [56, 57, 59]. Although $\beta$-defensins represent potentially a novel class of antimicrobial therapeutic agents, several obstacles must be overcome, including host cell cytotoxicity, degradation by proteases, loss of antimicrobial activity in the presence of a physiological concentration of salts, and high production cost due to their complex structure [83].

Via the characterization of the structure-function relationship of AvBDs and various analogs, it has been identified that the concentrated surface net positive charge and the Nterminal $\alpha$-helix and the $\beta 2-\beta 3$ loop structure are essential functional domains for antimicrobial and chemotactic properties [56]. Linear AvBD analogs with a high net positive charge (+9) and an N-terminal helix-loop possess improved antimicrobial potency and partial chemotactic activity, compared to wild-type AvBD-12 [56]. However, the linear AvBDs designed in our previous study are still sensitive to physiological salt conditions and the length of peptides (45 amino acid residues) remain to be shortened to control the manufacturing cost. In addition, a previous study has indicated that linear peptides are more 
susceptible to protease degradation due to lack of complex tertiary structure stabilized by disulfide bonds found in natural defensin peptides [165].

To increase salt- and protease-resistance, several solutions have been proposed, including: incorporating nonproteinogenic amino acids (e.g. D-amino acid substitutions and bulky amino acid $\beta$-naphthylalanin) $[89,166]$ or LPS binding peptide motif ( $\beta$-boomerang motif GWKRKRFG) [167], modifying the terminal regions via covalent linkage of a hydrophobic moiety (e. g. sterols or fatty acids) [168, 169], peptidomimetic [170], altering the structure, charge, hydrophobicity, and shortening the length of the peptide $[171,172]$. These strategies successfully improved the antimicrobial function of CAMPs, but resulted in elevated hemolytic activity and increased manufacturing cost $[89,173]$. In the present study, an integrated approach was utilized to design short and compositionally simple CAMPs with potent antimicrobial activity, improved resistance to salts and proteases and minimal cytotoxicity to host cells. The antibacterial property of the newly designed CAMPs against $P$. aeruginosa and Staphylococcus spp., including clinical isolates of multidrug-resistant $P$. aeruginosa and methicillin-resistant $S$. pseudintermedius (MRSP) was assessed under various conditions.

\section{Methods and Materials}

\section{Bacterial strains and cultures}

P. aeruginosa (ATCC 27853) and S. aureus (ATCC 29213) were used to evaluate the novel CAMPs' antimicrobial activity, salt- and protease-resistance, and membrane permeability. Ten multiple-drug resistant $P$. aeruginosa clinical isolates and ten methicillin-resistant $S$. pseudintermedius (MRSP) clinical isolates (Table 2) were obtained from the Veterinary Medical Diagnostic Laboratory at University of Missouri and subjected to an antimicrobial 
study of new CAMPs. All bacterial strains were maintained and grown in LB broth or agar $\left(\mathrm{BD}\right.$ Difco $^{\mathrm{TM}}$ ) at $37{ }^{\circ} \mathrm{C}$ as described previously [56, 59].

\section{Peptide synthesis and characteristics}

All peptides were custom synthesized using the standard solid phase 9fluorenylmethoxycarbonyl (Fmoc) method as previously synthesizing wild-type AvBDs [59] and purified by reverse phase high-performance liquid chromatography (RP-HPLC). The purity of the synthetic CAMPs was greater than $98.5 \%$ as verified by liquid chromatography-mass spectrometry (LC-MS) (Lifetein, Hillsborough, NJ).

The charge and hydrophobicity of the newly designed CAMPs at neutral $\mathrm{pH}$ were calculated using online Peptide property calculator (PepCalc.com). Protease cutting sites were predicted by using PROSPER (https://prosper.erc.monash.edu.au) and SignalP 4.1 server (http://www.cbs.dtu.dk/services/SignalP/). The three-dimensional structures of AvBDs and the newly designed templates were analyzed by using the I-TASSER protein structure and function prediction program (http://zhanglab.ccmb.med.umich.edu/ITASSER). The distribution of selected amino acid residues was evaluated using PyMOL, a user-sponsored molecular visualization system (https://www.pymol.org/).

\section{Circular Dichroism Spectrum Analysis}

Peptide structures were examined by far-UV circular dichroism (CD) spectroscopy with an Aviv Model 62DS spectrometer (Lakewood, NJ), in the wavelengths ranging from 190 to $250 \mathrm{~nm}$ using a path length of $1 \mathrm{~mm}$. The CD spectra of peptides were measured at a concentration of $0.15 \mathrm{mg} / \mathrm{ml}$ in water. Spectra were baseline corrected by subtracting a blank spectrum of buffer and expressed as molar ellipticity $\theta\left(\mathrm{deg} \cdot \mathrm{cm}^{2} \cdot \mathrm{mol}^{-1}\right)$.

\section{Membrane permeabilizing assay}


The membrane permeabilizing ability was determined using the propidium iodide (PI) uptake assay [174]. PI is a fluorescent molecule that can only penetrate the impaired microbial membrane and intercalate double-stranded DNA. PI staining was done according to the manufacturer's instruction (Sigma Aldrich). In brief, the mid-logarithmic culture of P. aeruginosa (ATCC 27853) was harvested by centrifugation at $1000 \times g$ for $10 \mathrm{~min}$ and resuspended in PBS $\left(1 \times 10^{8} \mathrm{CFU} / \mathrm{ml}\right)$. The bacteria were treated with each CAMP at a concentration of $1 \times \mathrm{MIC}$ for $15,30,60$, and 90 min, respectively. After the addition of PI, the suspension was further incubated for $5 \mathrm{~min}$ at room temperature and shielded from light. The bacterial mixture was coated on a microscope slide for analysis of red fluorescence. Images were captured using a Nikon fluorescent microscope with Olympus DP2-BSE software (Eclipse E600, Japan) and the number of fluorescent cells per field was counted by the ImageJ software (NIH, Bethesda, MD). The assay was performed in triplicate.

\section{Antimicrobial activity}

The minimum inhibitory concentrations (MICs) of CAMPs were determined according to the guidelines of the Clinical and Laboratory Standards Institute (CLSI) [117, 118]. The Muller Hinton (MH) II broth used in MIC assay was supplemented with $20-25 \mathrm{mg} / \mathrm{L}$ of calcium and 10-12.5 mg/L of magnesium. In brief, peptides were two-fold serially diluted ( 2 to $256 \mu \mathrm{g} / \mathrm{ml}$ ) in a 96-well microtiter plate and an equal volume $(\mu \mathrm{l})$ of bacterial suspension was added to each well of the plate. The final bacterial concentration in the wells was $5 \times 10^{5} \mathrm{CFU} / \mathrm{ml}$. The plate was incubated at $37{ }^{\circ} \mathrm{C}$ for $24 \mathrm{~h}$, and the lowest concentration that completely inhibited visible bacterial growth was recorded. All assays were conducted in triplicate.

\section{Salt resistance assay}


A major hindrance to clinical application of defensin peptides is the interference of function by cationic salts, often referred to as salt sensitivity [76]. The effect of salt on antimicrobial activity of novel CAMPs against $P$. aeruginosa ATCC 27853 and S. aureus ATCC 29213 was determined by a colony count assay [59], in the presence of either $0,50,100$, and 150 $\mathrm{mM} \mathrm{NaCl}$ or $0,0.5,1$, and $2 \mathrm{mM} \mathrm{CaCl}_{2}$. In brief, bacterial colonies from an overnight culture plate were resuspended in assay medium (10-fold diluted Mueller Hinton II broth) to obtain a final concentration of $2 \times 10^{5} \mathrm{CFU} / \mathrm{ml}$. Twenty-five microliters of bacterial suspension and $25 \mu 1$ of CAMP were mixed in the wells of a 96-well polypropylene microtiter. Two peptide concentrations, $0.5 \times$ MIC and $1 \times$ MIC, were included in salt resistance assay and medium without CAMP served as a negative control. The bacteriapeptide mixtures were incubated at $37{ }^{\circ} \mathrm{C}$ for $2 \mathrm{~h}, 10$-fold serially diluted and plated on LB agar plates. The number of bacterial colonies was enumerated after $16 \mathrm{~h}$ of incubation at $37{ }^{\circ} \mathrm{C}$. Antimicrobial activity was expressed as percent of killing using the following formula: $\left(\mathrm{CFU}_{\text {control }}-\mathrm{CFU}_{\text {treated }}\right) / \mathrm{CFU}_{\text {control }} \times 100 \%$. All assays were performed in triplicate.

\section{Hemolytic assay}

The hemolytic assay was performed as described previously [173]. Briefly, mouse red blood cells (RBCs, Innovative Research, Novi, MI) were washed three times with phosphate-buffered saline (PBS, $\mathrm{pH} 7.4$ ), centrifuged at $1000 \times \mathrm{g}$ for $10 \mathrm{~min}$, and resuspended in PBS to $10 \%(\mathrm{v} / \mathrm{v})$. The RBCs were treated with CAMPs at various concentrations ranging from 4 to $512 \mu \mathrm{g} / \mathrm{ml}$ (2-fold serial dilutions) at $37{ }^{\circ} \mathrm{C}$ for $1 \mathrm{~h}$. PBS and $0.2 \%$ Triton $\mathrm{X}-100$ were used as negative and positive controls, respectively. The supernatant was transferred to a 96-well flat-bottomed polystyrene plate (Thermo Fisher Scientific), and the amount of hemoglobin released into the supernatant was determined by 
measuring the absorbance with a spectrophotometer at $540 \mathrm{~nm}$. Hemolytic activity was expressed as the percentage of hemolysis and calculated using the following equation: hemolysis $(\%)=\left(\mathrm{A}_{\mathrm{s}}-\mathrm{A}_{0}\right) /\left(\mathrm{A}_{100}-\mathrm{A}_{0}\right) \times 100$, where $\mathrm{A}_{\mathrm{s}}$ is the absorbance of the sample, $\mathrm{A}_{100}$ is the absorbance of completely lysed RBCs in $0.2 \%$ Triton $\mathrm{X}-100$, and $\mathrm{A}_{0}$ is the absorbance in the complete absence of hemolysis (PBS treatment). The assay was performed in triplicate. The therapeutic index (T.I.) was calculated according to a previously published formula: T.I. = MHC / $\mathrm{MIC}_{\mathrm{GM}}$ [175]. $\mathrm{MHC}$ was the minimum hemolytic concentration that caused $5 \%$ hemolysis of mouse RBCs. The MIC $\mathrm{GM}_{\mathrm{G}}$ was the minimum inhibitory concentration of the peptide concentrations against bacterial growth after the geometric mean was calculated. $\mathrm{MIC}_{\mathrm{G}-\text { was the }} \mathrm{MIC}_{\mathrm{GM}}$ for Gram-negative bacteria; $\mathrm{MIC}_{\mathrm{G}+}$ was the $\mathrm{MIC}_{\mathrm{GM}}$ for Gram-positive bacteria.

\section{Cell culture and cell cytotoxicity}

Mouse immature dendritic cell line JAWSII (ATCC CRL-11904 ${ }^{\mathrm{TM}}$ ) cells and CCR-2 transfected CHO-K1 cells were cultured as described in Chapter 3. The cytotoxicity of CAMP peptides to JASWII and CHO-K1 cells was determined using MTT (3-(4, 5dimethylthiazol-2-yl)-2, 5-diphenyltetrazolium bromide, Thermo Fisher Scientific) cell proliferation assay [59], with peptides at $64,128,256$, and $512 \mu \mathrm{g} / \mathrm{ml}$ for $4,12,24$, and 48 h at $37^{\circ} \mathrm{C}$. The experiment was performed in triplicate.

\section{Chemotaxis assay}

Migration of JAWSII and CCR-2 transfected CHO-K1 cells in response to CAMPs was determined using a 48-well microchemotaxis chamber technique as previously described [59, 119]. Chemotaxis buffer (Minimum Essential Medium containing 0.1\% BSA, 100U/ml penicillin, and $100 \mu \mathrm{g} / \mathrm{ml}$ streptomycin) and bacterial peptide $\mathrm{N}$-formyl- 
methionyl-leucyl-phenylalanine (fMLF, Sigma-Aldrich) were included as negative and positive controls, respectively. The results were presented as chemotactic indexes (C.I.) = number of migrated cells induced by AvBDs / number of migrated cells induced by the chemotactic buffer. The assay was repeated five times.

\section{Protease resistance assay}

Protease resistance was evaluated by using sodium dodecyl sulfate-polyacrylamide gel electrophoresis (SDS-PAGE) followed by antimicrobial assays. CAMP peptides $(10 \mu \mathrm{g})$ were treated with various proteases at various concentrations comparable to or higher than that found in host or bacterial culture, including $0.12,0.6$, and $1.2 \mu \mathrm{g} / \mathrm{ml}$ of $\alpha$-chymotrypsin (Thermo Fisher Scientific) [173], 0.4, 4.4, and $20 \mu \mathrm{g} / \mathrm{ml}$ of matrilysin (metalloproteinase7, Sigma Aldrich) [176], 0.2, 2, and $20 \mu \mathrm{g} / \mathrm{ml}$ elastase (Thermo Fisher Scientific) [177], or $0.2,2$, and $20 \mu \mathrm{g} / \mathrm{ml}$ of cathepsin B (Thermo Fisher Scientific) [178]. Protease digestion assay was carried out in $20 \mu \mathrm{l}$ of digestion buffer ( $25 \mathrm{mM}$ Tris and $150 \mathrm{mM} \mathrm{NaCl}, \mathrm{pH} 7.8$ ) for 1 hour at $37^{\circ} \mathrm{C}$. After treatment, the digestion mixture was analyzed by SDS-PAGE on 16.5\% polyacrylamide gel. To determine the effect of protease digestion on the antimicrobial activity of two most active CAMPs, each peptide was first treated with a protease for $1 \mathrm{~h}$ at $37{ }^{\circ} \mathrm{C}$. The mixture was then diluted to $1 \times \mathrm{MIC}$ of the peptide and subjected to colony count assay as described above. Assay buffer containing protease but not peptide and buffer containing untreated peptide were included as controls. Protease inhibition of the antimicrobial activity of CAMP peptide was expressed as a percentage of killing by protease treated peptide in relevance to the untreated peptide. The experiment was repeated three times in triplicate in each assay.

\section{Statistical analysis}


Data presented were the means \pm standard deviation $(\mathrm{SD})$. Differences between groups were analyzed using the one-way analysis of variance (ANOVA) followed by Duncan's test for multiple comparisons using software SPSS version 19.0 (IBM Corp., Armonk, NY). Differences at $p<0.05$ level were considered statistically significant, and at $p<0.01$ level were considered extremely significant.

\section{Results}

\section{Peptide design}

Initially, two CAMP templates were designed to retain the antimicrobial and chemotactic properties of wild-type AvBD-6 and AvBD-12, respectively. The first CAMP template (CAMP-t1) possessed the structural domain (N-terminal $\alpha$-helix and $\beta 2-\beta 3$ loop) of AvBD12 as well as its analogs A2 and A3 which appeared to be essential to the broad chemotactic activity of AvBD-12 [56]. To increase the net positive charge, the negatively charged amino acid residues Asp (D) and Glu (E) were substituted with positively charged amino acid residues Lys $(\mathrm{K})$ or Arg (R). Trp (W) amino acid residue has an aromatic ring which makes it have a strong tendency to insert into membrane lipid bilayer and Trp-rich peptides exhibit enhanced antimicrobial activity and salt resistance [89]. CAMP-t1 consisted of 25 amino acid residues: RKFLRRRGEVAHFSQKSLGLYCWWW. The poly-Trp (W) tail was incorporated to enhance salt-resistance of CAMP-t1. As shown in Figure 4-1A, the predicted three-dimensional structure of CAMP-t1 mimics that of AvBD-12. The second CAMP template (CAMP-t2) consisted of key amino acid residues of AvBD-6: PIHRRIPPRWPRLKRRW, contributing to the concentrated surface charge and hydrophobicity of AvBD-6 [56]. CAMP-t2 assumed a coil and $\alpha$-helix structure as shown in Figure 4-1B. To optimize the antimicrobial function, CAMP-t2 was subjected to further 
modifications using the following criteria: (1) short in length ( $\leq 20$ a.a. residues), $(2)$ the hydrophobic residues on one side and hydrophilic residues on the opposite side to facilitate pore formation by multiple peptides in bacterial membrane [8], (3) lack of cutting sites for four major protease families: aspartic protease, cysteine protease, metalloprotease, serine proteases, (4) N-terminal acetylation and C-terminal amidation to increase the metabolic stability of CAMPs [179], and (5) addition of C-terminal poly-Trp tail to enhance membrane permeabilization [180]. The resulting peptides form CAMP-t2, CAMP-A and CAMP-B, assumed the $\alpha$-helix structure as shown in Figure 4-1C and 1D. Their net positive charge amino acid residues were aligned at one side of the helical surface and the C-termini were coated with poly-Trp tails.

\section{Structural features of CAMPs}

The amino acid sequences and relevant biochemical characteristics of all CAMPs were presented in Table 4-1. The structures of CAMPs was analyzed by a far-UV spectrometer. The random coil or unordered peptides usually show a single band below $200 \mathrm{~nm}$, whereas peptide with alpha-helical structures usually presents two negative bands at 208 and 222 $\mathrm{nm}$ along with one positive band at $192 \mathrm{~nm}$ in CD spectrum [181]. The CD spectrum of CAMP-t1 showed two small signals at $202 \mathrm{~nm}$ and $225 \mathrm{~nm}$, indicating only partial helical structures (Figure 4-2). CAMP-t2 only displayed a single band around $200 \mathrm{~nm}$, indicating

a random structure, as predicted in Figure 4-1B. The CD spectrum of CAMP-A displayed two big negative signals at $200 \mathrm{~nm}$ and $225 \mathrm{~nm}$ and a positive signal at $190 \mathrm{~nm}$, indicating a helix structure. The CD spectrum of CAMP-B showed two small signals at $202 \mathrm{~nm}$ and $225 \mathrm{~nm}$, indicating short helical structures. 
A
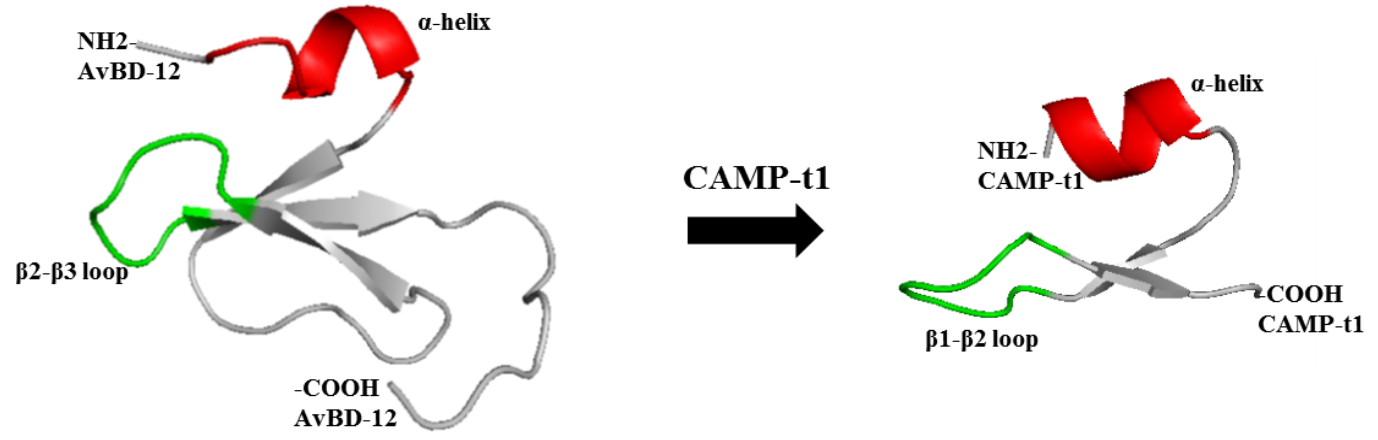

GPDSCNHDRGLCRVGNCNPGEYLAKYCFEPVILCCKPLSPTPTKT

RKFLRRGEVAHFSQKSLGLYCWWW
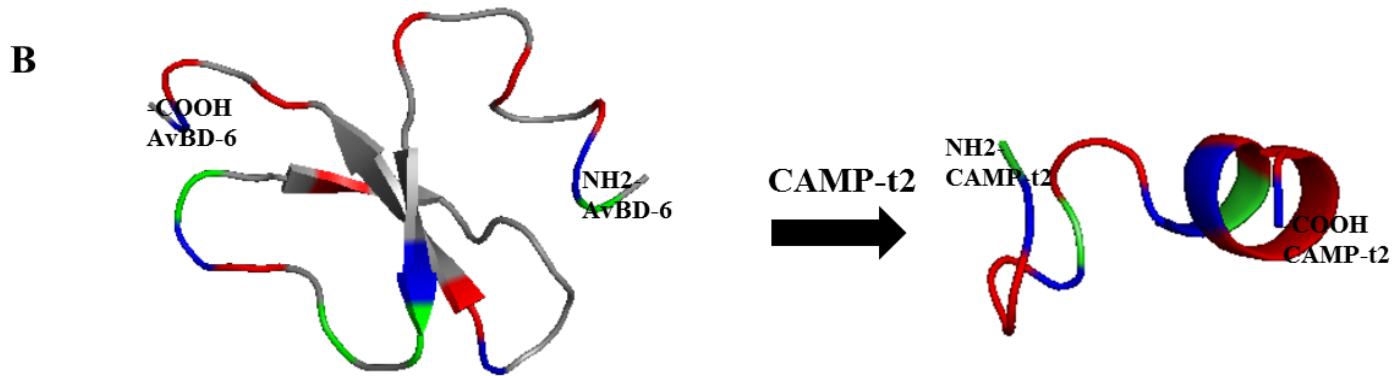

SPIHACRYQRGVCIPGPCRWPYYRVGSCGSGLKSCCVRNRWA

PIHRRIPPRWPRLKRRW

C

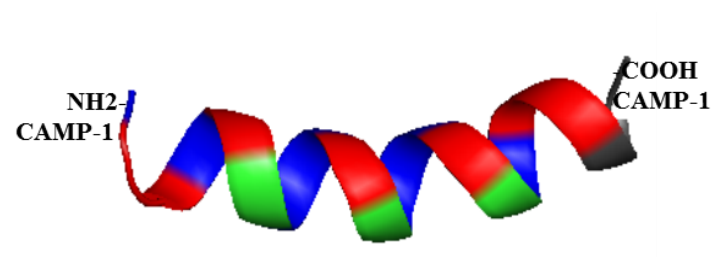

LRRLKPLIRPWLRPLRRWWW
D

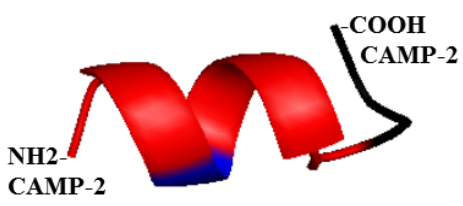

RRRWRKRRWWW

Figure 4-1. The predicted structures of templates CAMP-t1 and CAMP-t2

(A) The three-dimensional structure of CAMP-t1 derived from AvBD-12. Red: $\alpha$-helix; Green: loop ( $\beta 2-\beta 3$ loop in AvBD-12, $\beta 1-\beta 2$ loop in template CAMP-t1). (B) The threedimensional structure of CAMP-t2 derived from AvBD-6. CAMP-t2 were further optimized to CAMP-A (C) and CAMP-B (D). CAMP-A and CAMP-B were coated with poly-Trp tails. Red: positively charged amino acid residues; Blue: hydrophobic amino acid residues; Green: prolines. 


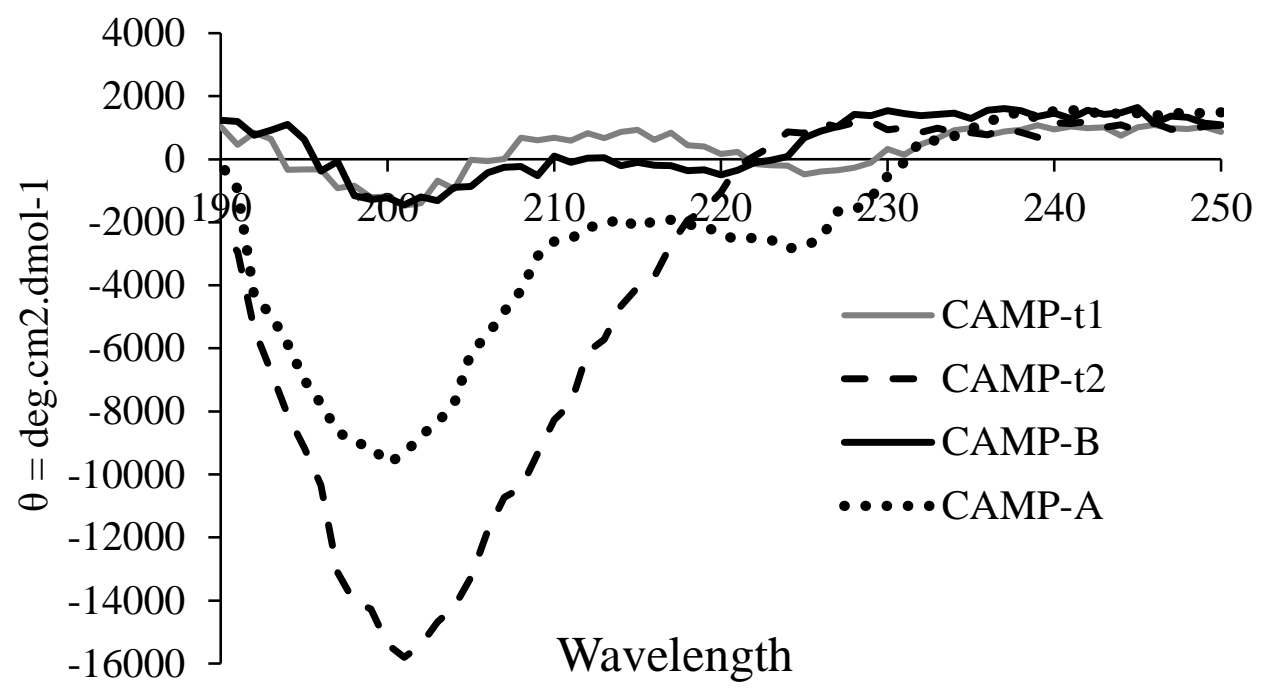

Figure 4-2. The far-UV CD spectra of CAMPs in $\mathrm{H}_{2} \mathrm{O}$ recorded at room temperature Spectra were baseline corrected and expressed as molar ellipticity $\theta\left(\mathrm{deg} \cdot \mathrm{cm}^{2} \cdot \mathrm{mol}^{-1}\right)$. Gray solid line: CAMP-t1; dash line: CAMP-t2; dotted line: CAMP-A; dark solid line: CAMPB.

Table 4-1. The characteristics of newly designed CAMPs

\begin{tabular}{llcccc}
\hline Peptide* & Amino acid sequence & $\begin{array}{c}\text { Length } \\
\text { (aa) }\end{array}$ & $\begin{array}{l}\text { Molecular } \\
\text { Weight }\end{array}$ & Charge & Hydrophobicity \\
\hline CAMP-t1 & $\begin{array}{l}\text { RKFLRRRGEVAHFS } \\
\text { QKSLGLYCWWW }\end{array}$ & 25 & 3251.84 & +4 & $44 \%$ \\
CAMP-t2 & $\begin{array}{l}\text { PIHRRIPPRWPRLK } \\
\text { RRW }\end{array}$ & 17 & 2361.90 & +7 & $29 \%$ \\
CAMP-A & $\begin{array}{l}\text { LRRLKPLIRPWLRP } \\
\text { LRRWWW }\end{array}$ & 20 & 2839.55 & +7 & $50 \%$ \\
CAMP-B & RRRWRKRRWWW & 11 & 1869.24 & +7 & $36 \%$ \\
\hline
\end{tabular}

*CAMP-t1, CAMP-t2, and CAMP-B are coated with a Trp-tail. N-terminal acetylation and C-terminal amidation were introduced to all peptides. 


\section{Antimicrobial activity}

The minimum inhibitory concentrations of the newly designed CAMPs against $P$. aeruginosa and $S$. aureus ATCC reference strains and multidrug-resistant $P$. aeruginosa and methicillin-resistant $S$. pseudinetermedius (MASP) strains were compared to that of AvBD-6, a natural host CAMP with potent antimicrobial activity under low salt conditions (Table 4-2). CAMP-t1 showed significantly improved anti-Pseudomonas activity with MIC values 4-fold (for ATCC reference strain) and 2-fold (clinical isolates) lower than that of AvBD-6. CAMP-t1 showed improved antimicrobial activity against ATCC S. aureus reference strain with MIC value that was 4-fold lower than that of AvBD-6, but not MRSP clinical isolates as evidenced by the high MIC values similar to that of AvBD-6. CAMP$\mathrm{t} 2$, the shorter template, demonstrated similar antimicrobial activity against Pseudomonas strains and significantly enhanced anti-Staphylococcus activity, compared to CAMP-t1. The MICs of CAMP-t2 against S. aureus and MRSP strains were up to 4-fold lower than that of CAMP-t1 and AvBD-6. CAMP-A, a derivative of CAMP-t2, established further improved antimicrobial activity against $P$. aeruginosa and $S$. aureus reference strains, multidrug-resistant $P$. aeruginosa strains and MRSP clinical isolates. The MICs of CAMPA against $P$. aeruginosa and MRSP isolates were 4- to 32- fold lower than that of AvBD6. CAMP-B, the second derivative of CAMP-t2, also showed improved antimicrobial activity against $P$. aeruginosa and similar potency against Staphylococcus spp. However, CAMP-B was less effective than CAMP-A in killing both Pseudomonas spp. and methicillin-resistant S. pseudintermedius ( $p<0.05$, Table 4-2). 
Table 4-2. The minimum inhibitory concentration (MIC) of CAMPs

\begin{tabular}{|c|c|c|c|c|c|}
\hline CAMPs & CAMP-t1 & CAMP-t2 & CAMP-A & CAMP-B & AvBD-6 \\
\hline $\begin{array}{l}\text { Bacteria } \\
\text { (Strain identification) }\end{array}$ & $\begin{array}{c}\mathrm{MIC} \\
(\mu \mathrm{g} / \mathrm{ml})\end{array}$ & $\begin{array}{c}\mathrm{MIC} \\
(\mu \mathrm{g} / \mathrm{ml})\end{array}$ & $\begin{array}{c}\mathrm{MIC} \\
(\mu \mathrm{g} / \mathrm{ml})\end{array}$ & $\begin{array}{c}\mathrm{MIC} \\
(\mu \mathrm{g} / \mathrm{ml})\end{array}$ & $\begin{array}{c}\mathrm{MIC} \\
(\mu \mathrm{g} / \mathrm{ml})\end{array}$ \\
\hline $\begin{array}{l}\text { P. aeruginosa } \\
\text { (ATCC 27853) }\end{array}$ & 64 & 64 & 16 & 32 & $>256$ \\
\hline $\begin{array}{l}\text { P. aeruginosa } \\
(1704173)^{\#}\end{array}$ & 128 & 64 & 16 & 32 & $>256$ \\
\hline $\begin{array}{l}\text { P. aeruginosa } \\
(1703357)^{\#}\end{array}$ & 128 & 128 & 16 & 64 & $>256$ \\
\hline $\begin{array}{l}\text { P. aeruginosa } \\
(1703511)^{\#}\end{array}$ & 128 & 128 & 16 & 64 & $>256$ \\
\hline $\begin{array}{l}P . \text { aeruginosa } \\
(1703000)^{\#}\end{array}$ & 128 & 64 & 8 & 32 & $>256$ \\
\hline $\begin{array}{l}P . \text { aeruginosa } \\
(1703002)^{\#}\end{array}$ & 128 & 256 & 16 & 64 & $>256$ \\
\hline $\begin{array}{l}P . \text { aeruginosa } \\
(1703451)^{\#}\end{array}$ & 128 & 64 & 16 & 64 & $>256$ \\
\hline $\begin{array}{l}P . \text { aeruginosa } \\
(1703949)^{\#}\end{array}$ & 128 & 128 & 16 & 64 & $>256$ \\
\hline $\begin{array}{l}P . \text { aeruginosa } \\
(1703290)^{\#}\end{array}$ & 128 & 128 & 16 & 64 & $>256$ \\
\hline $\begin{array}{l}\text { P. aeruginosa } \\
(1703983)^{\#}\end{array}$ & 128 & 256 & 16 & 64 & $>256$ \\
\hline $\begin{array}{l}P . \text { aeruginosa } \\
(1704175)^{\#}\end{array}$ & 128 & 64 & 16 & 32 & $>256$ \\
\hline $\mathrm{MIC}_{\text {Average G- }}$ & $\begin{array}{c}122.18 \pm \\
19.29^{\mathrm{a}} \\
\end{array}$ & $\begin{array}{c}122.18 \pm \\
72.71^{\mathrm{a}} \\
\end{array}$ & $\begin{array}{c}15.27 \pm \\
2.41^{\mathrm{c}} \\
\end{array}$ & $\begin{array}{c}52.36 \pm \\
16.14^{\mathrm{b}} \\
\end{array}$ & $>256$ \\
\hline $\begin{array}{l}\text { S. aureus } \\
\text { (ATCC 29213) }\end{array}$ & 64 & 64 & 32 & 32 & 256 \\
\hline $\begin{array}{l}\text { S. pseudintermedius } \\
(13164006)^{*}\end{array}$ & 256 & 32 & 16 & 32 & $>256$ \\
\hline $\begin{array}{l}\text { S. pseudintermedius } \\
(13203008)^{*}\end{array}$ & 256 & 32 & 16 & 64 & 256 \\
\hline $\begin{array}{l}\text { S. pseudintermedius } \\
(13178007)^{*}\end{array}$ & 256 & 64 & 16 & 64 & 256 \\
\hline $\begin{array}{l}\text { S. pseudintermedius } \\
(13267017)^{*}\end{array}$ & 128 & 32 & 16 & 32 & $>256$ \\
\hline $\begin{array}{l}\text { S. pseudintermedius } \\
(13252001)^{*}\end{array}$ & 64 & 32 & 16 & 32 & 256 \\
\hline
\end{tabular}




\begin{tabular}{|c|c|c|c|c|c|}
\hline $\begin{array}{l}\text { S. pseudintermedius } \\
(13269013)^{*}\end{array}$ & 256 & 32 & 32 & 32 & $>256$ \\
\hline $\begin{array}{l}\text { S. pseudintermedius } \\
(13193006)^{*}\end{array}$ & 256 & 128 & 32 & 32 & $>256$ \\
\hline $\begin{array}{l}\text { S. pseudintermedius } \\
(13228005)^{*}\end{array}$ & 256 & 32 & 16 & 64 & $>256$ \\
\hline $\begin{array}{l}\text { S. pseudintermedius } \\
(13207007)^{*}\end{array}$ & 256 & 32 & 16 & 64 & $>256$ \\
\hline $\begin{array}{l}\text { S. pseudintermedius } \\
\text { (13250111)* }^{*}\end{array}$ & 256 & 32 & 32 & 64 & 256 \\
\hline $\mathrm{MIC}_{\text {Average G+ }}$ & $\begin{array}{c}209.45 \pm \\
81.41^{\mathrm{a}}\end{array}$ & $\begin{array}{l}46.55 \pm \\
29.89^{b}\end{array}$ & $\begin{array}{l}21.82 \pm \\
8.07^{\mathrm{c}}\end{array}$ & $\begin{array}{l}46.55 \pm \\
16.71^{\mathrm{b}}\end{array}$ & $\geq 256$ \\
\hline
\end{tabular}

${ }^{\#}$ Multidrug resistant clinical isolates of P. aeruginosa were resistant to chloramphenicol, tetracycline, sulfamethoxazole, and $\beta$-lactam antibiotics: amoxicillin, ampicillin, and cefazolin. *Methicillin-resistant S. pseudintermedius (MRSP). Superscripts a, b, and c mean significant difference $(p<0.05)$ between MICs of four CAMPs against either Gramnegative or Gram-positive bacteria. 


\section{Membrane permeabilizing activity}

A propidium iodide (PI) uptake assay was carried out to determine the membrane permeabilizing activity of newly designed CAMPs (Figure 4-3). As shown in Figure 4-3A, CAMP-treated $P$. aeruginos $a$ were stained red, indicating that bacterial membranes were damaged by CAMPs. In contrast, untreated bacteria did not show red fluorescence. When the permeabilizing ability was assessed based on the numbers of red cells per field, a timedependent increase was observed from 15 to 90 min (Figure 4-3B and 3C). However, the numbers of red bacteria did not increase significantly after $30 \mathrm{~min}$, indicating a fast-action mode of CAMPs. Similar results were obtained for all CAMPs and both bacterial pathogens (Figure 4-3). 

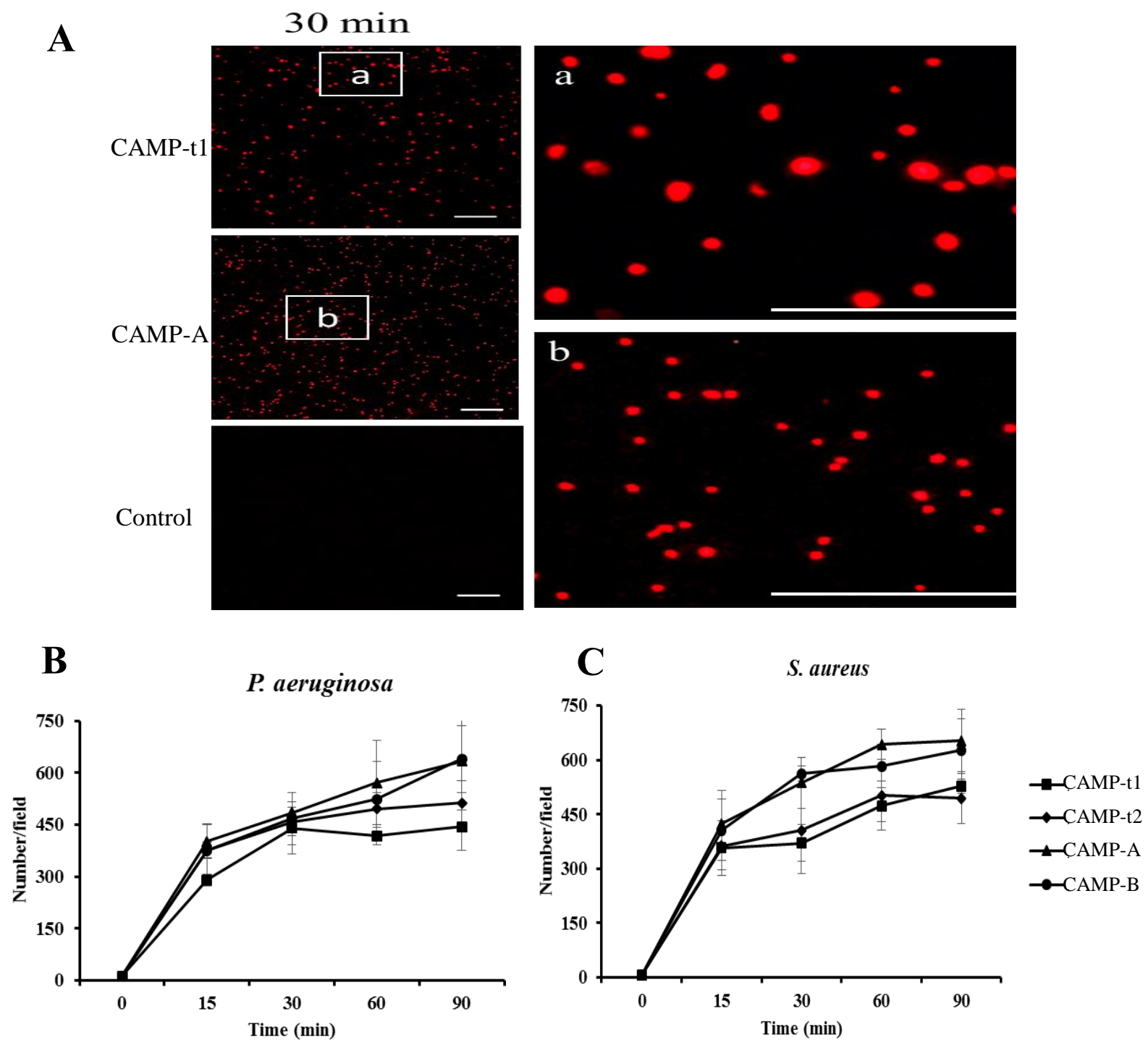

Figure 4-3. Membrane permeabilizing activity of CAMPs

(A) Representative fluorescence microscopy images of CAMP-treated and control bacteria stained with membrane-impermeable DNA dye propidium iodide (PI). Right panels are enlarged focal areas ( $a$ and $b$ ) from the left panels. (B) The number per field of positively stained $P$. aeruginos $a$ at various times post-CAMP treatment. (C) The number per field of S. aureus at various times post-CAMP treatment. Data are expressed as the means $\pm \mathrm{SD}$ $(\mathrm{n}=3)$. An asterisk indicates a significant difference between different time points $\left({ }^{*} p<\right.$ 0.05). Bar: $100 \mu \mathrm{m}$. 


\section{Salt resistance}

The impact of cationic salts on the bactericidal activity of CAMPs at two peptide concentrations $(1 \times \mathrm{MIC}$ and $0.5 \times \mathrm{MIC})$ was assessed in conditions of various concentrations of $\mathrm{NaCl}(0$ to $150 \mathrm{mM})$ or $\mathrm{CaCl}_{2}(0$ to $2 \mathrm{mM})$. As shown in Figure 4-4, increasing $\mathrm{NaCl}$ and $\mathrm{CaCl}_{2}$ concentrations had no impact on the bactericidal activity of CAMP-t1 which possesses a C-terminal poly-Trp tail. In contrast, the bactericidal activity of CAMP-B without the Trp tail was negatively affected by increased salt concentrations. At the physiological conditions of $\mathrm{NaCl}(100$ to $150 \mathrm{mM})$ and $\mathrm{CaCl}_{2}$ (1 to $\left.2 \mathrm{mM}\right), \mathrm{CAMP}-$ t2 retained approximately $40 \%$ of its killing activity against $P$. aeruginosa and $S$. aureus, compared to the results obtained at salt-free condition (Figure 4-4). The salt-resistance pattern of CAMP-t2 was similar at two different peptide concentrations. Similar to CAMPt1, CAMP-A and CAMP-B with a Trp tail exhibited strong tolerance to $\mathrm{NaCl}$ and $\mathrm{CaCl}_{2}$ (Figure 4-4).

\section{Hemolytic activity}

The hemolytic activity of CAMPs to mouse RBCs was measured (Figure 4-5). CAMP-A caused average $3.6 \%, 10.1 \%$, and $17.5 \%$ of mRBCs lysis at the concentration of $128 \mu \mathrm{g} / \mathrm{ml}$, $256 \mu \mathrm{g} / \mathrm{ml}$, and $512 \mu \mathrm{g} / \mathrm{ml}$, respectively. In contrast, CAMP-t1, CAMP-t2, and CAMP-B did not cause more than $5 \%$ of mRBCs lysis at the concentration of $512 \mu \mathrm{g} / \mathrm{ml}$. The minimum hemolytic concentration $(\mathrm{MHC})$, geometric means of the $\mathrm{MIC}\left(\mathrm{MIC}_{\mathrm{GM}}\right)$, and therapeutic index (T.I.) were determined for each CAMP (Table 4-3). The average T.I. of CAMP-A and CAMP-B against $P$. aeruginosa were $8.72 \pm 2.41$ and >10.90 \pm 4.0 , respectively. The T.I. of CAMP-A and CAMP-B against S. aureus were $6.54 \pm 2.01$ and $>12.36 \pm 4.18$, respectively. 


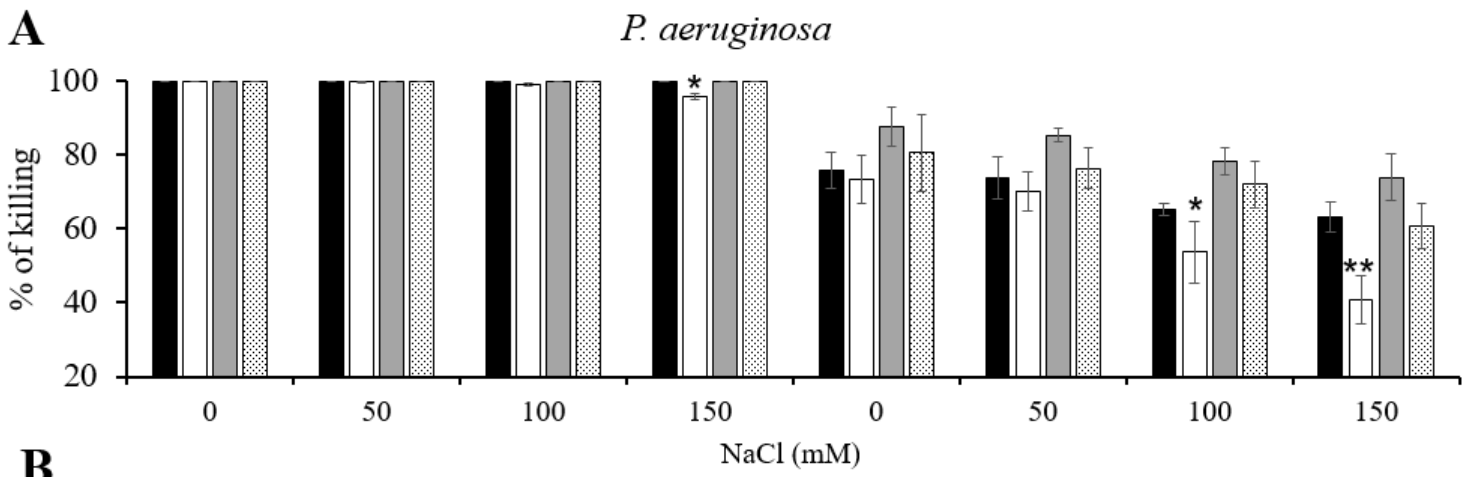

B

S. aureus
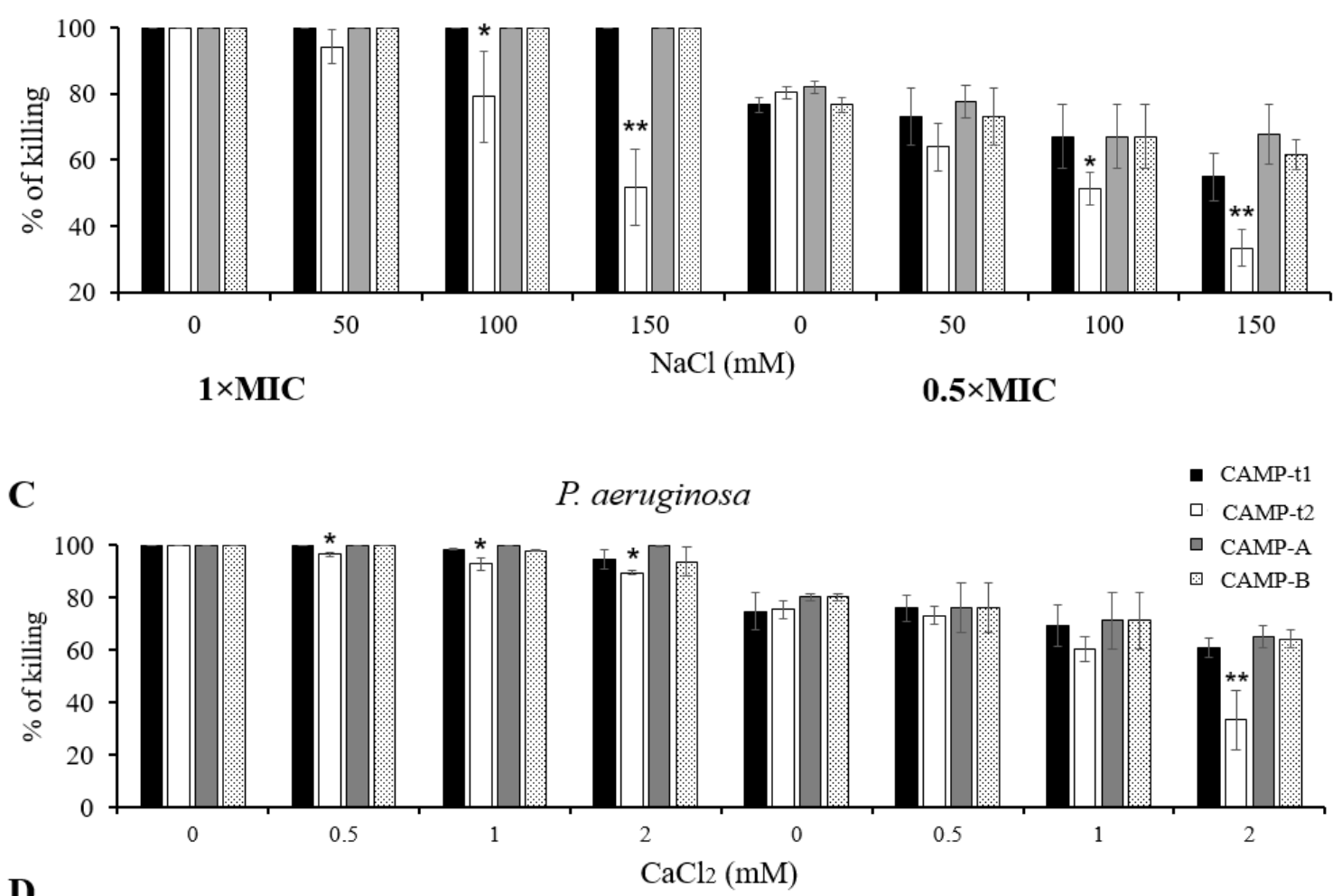

D

S. aureus

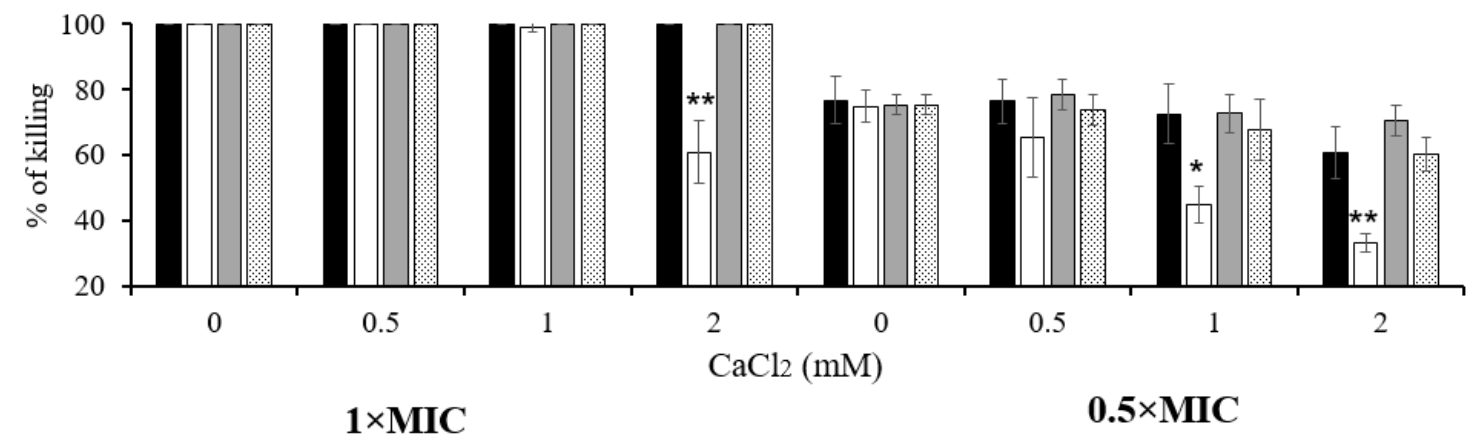

Figure 4-4. Effect of salts on the antibacterial activity of CAMPs against $P$. aeruginosa and $S$. aureus 
The effect of $\mathrm{NaCl}$ and $\mathrm{CaCl}_{2}$ on the antibacterial activity of four CAMPs was determined at two peptide concentrations, $1 \times$ MIC and $0.5 \times$ MIC. The killing percent of four CAMPs against $P$. aeruginosa (A) and against $S$. aureus (B) were tested in presence of $0,50,100$, and $150 \mathrm{mM} \mathrm{NaCl}$. The killing percent of four CAMPs against $P$. aeruginosa (C) and against $S$. aureus (D) were tested in presence of $0,0.5,1$, and $2 \mathrm{mM} \mathrm{CaCl}_{2}$. Data represent the means \pm SD of three independent experiments. An asterisk indicates a statistically significant difference between antimicrobial activity in the presence and absence of salts $(* p<0.05$ and $* * p<0.01)$

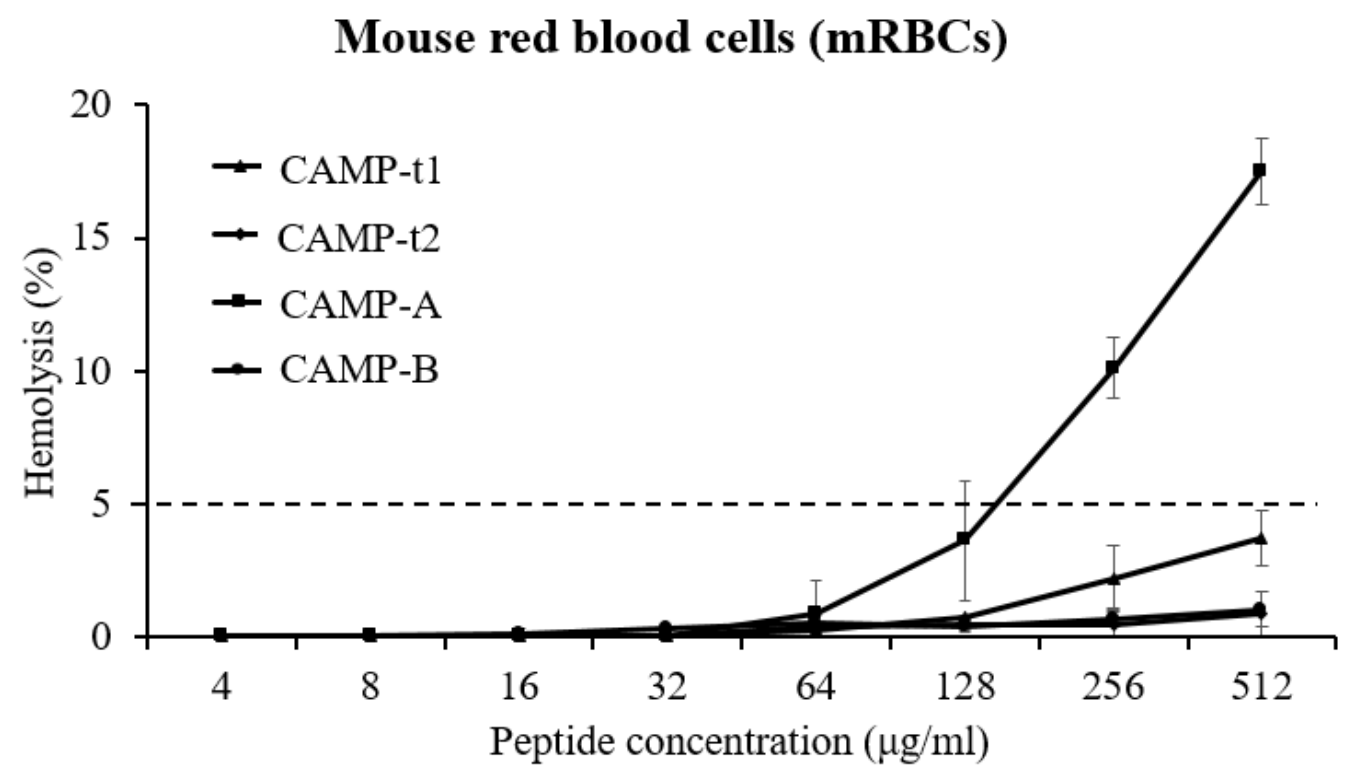

Figure 4-5. Hemolytic activity of CAMPs

CAMP-induced hemolysis (\%) of mouse red blood cells at various peptide concentrations is defined as a percentage of complete hemolysis caused by $0.2 \%$ Triton X-100. Data are expressed as the means \pm SD of three independent experiments. 
Table 4-3. The therapeutic index of new cationic antimicrobial peptides (CAMPs)

\begin{tabular}{c|c|c|c|c}
\hline Peptides & CAMP-t1 & CAMP-t2 & CAMP-A & CAMP-B \\
\hline MHC & $>512$ & $>512$ & 128 & $>512$ \\
MIC $_{\text {Average G- }}$ & $122.18 \pm 19.29$ & $122.18 \pm 72.71$ & $15.27 \pm 2.41$ & $52.36 \pm 16.14$ \\
MIC $_{\text {Average G+ }}$ & $209.45 \pm 81.41$ & $46.55 \pm 29.89$ & $21.82 \pm 8.07$ & $46.55 \pm 16.71$ \\
T.I. of G- & $>4.36 \pm 1.21$ & $>5.45 \pm 2.54$ & $8.72 \pm 2.41$ & $>10.90 \pm 4.0$ \\
T.I. of G+ & $>3.27 \pm 2.41$ & $>13.45 \pm 4.48$ & $6.54 \pm 2.01$ & $>12.36 \pm 4.18$ \\
MIC Average & $165.82 \pm 73.00$ & $84.36 \pm 66.65$ & $18.55 \pm 6.71$ & $49.45 \pm 11.64$ \\
T.I. Average & $>3.82 \pm 1.94$ & $>9.45 \pm 5.42$ & $7.64 \pm 2.44$ & $>11.64 \pm 4.08$ \\
\hline
\end{tabular}

The therapeutic index (T.I.) is defined as the ratio of $\mathrm{MHC}$ to $\mathrm{MIC}_{\mathrm{GM}}[175]$. $\mathrm{MHC}(\mu \mathrm{g} / \mathrm{ml})$ is the minimum hemolytic concentration that caused $5 \%$ hemolysis of mouse red blood cells (mRBCs). The $\mathrm{MIC}_{\mathrm{GM}}(\mu \mathrm{g} / \mathrm{ml})$ means the geometric mean $(\mathrm{GM})$ of the MIC values of the peptides against bacteria. $\mathrm{MIC}_{\mathrm{Average} \mathrm{G}}$ is the $\mathrm{MIC}_{\mathrm{GM}}$ for Gram-negative bacteria; $\mathrm{MIC}_{\text {Average } \mathrm{G}+}$ is the $\mathrm{MIC}_{\mathrm{GM}}$ for Gram-positive bacteria. $\mathrm{MIC}_{\text {Average }}$ is the $\mathrm{MIC}_{\mathrm{GM}}$ for both Gram-negative and Gram-positive bacteria. 
A

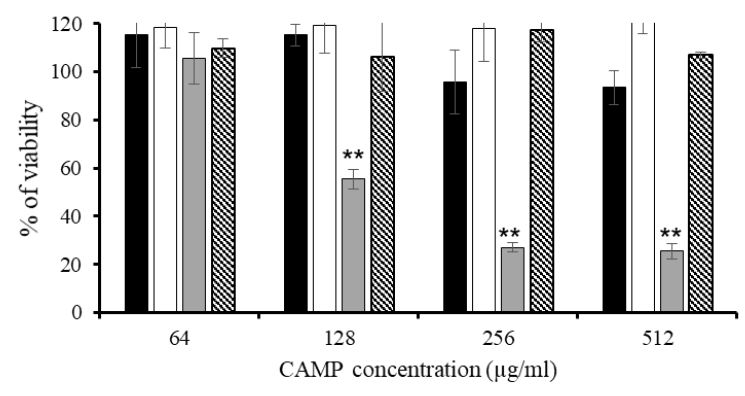

B

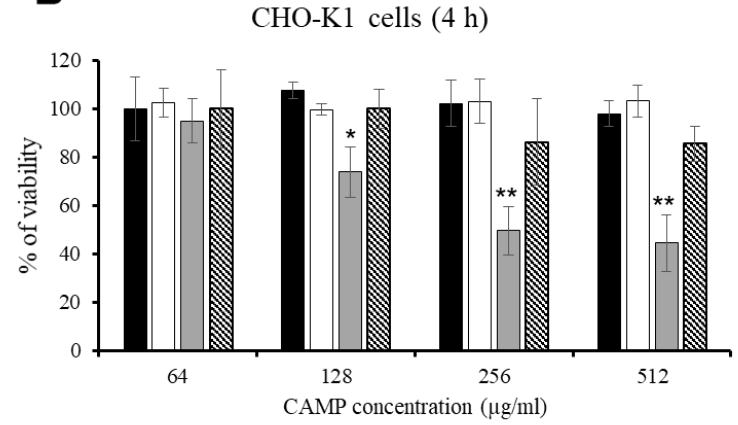

JAWSII cells (48 h)

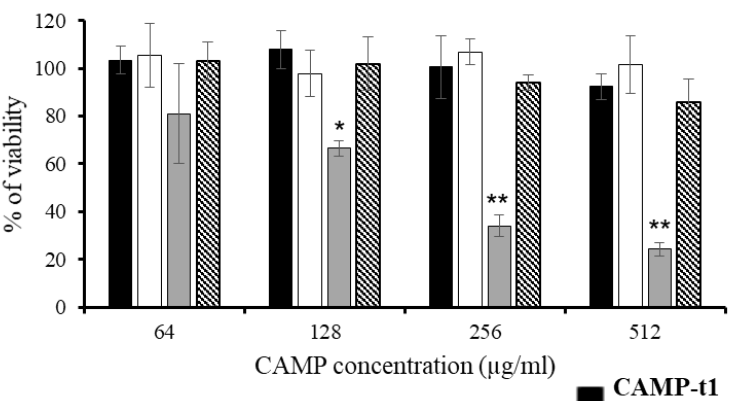

$\square$ CAMP-t2

CHO-K1 cells $(48 \mathrm{~h})$

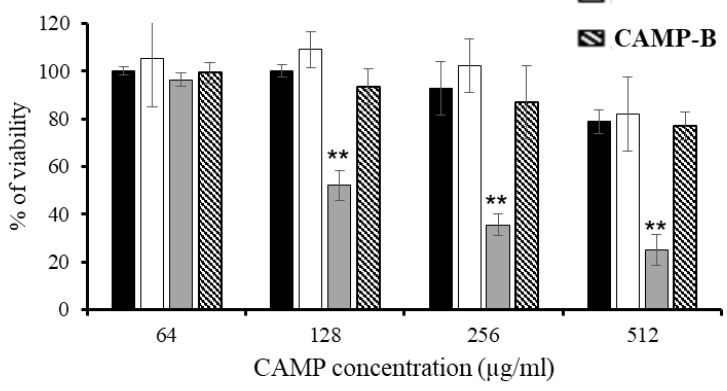

Figure 4-6. Cytotoxicity of CAMPs to JAWSII and CHO-K1 cells

Effect of CAMPs on the viability of mouse immature dendritic JAWSII cells (A) and hamster ovary $\mathrm{CHO}-\mathrm{K} 1$ cells (B) at 4 and $48 \mathrm{~h}$ of incubation with peptides at the concentration of 64 to $512 \mu \mathrm{g} / \mathrm{ml}$. Results are percentages of viable cells relative to the untreated control cells. The data are expressed as the mean \pm SD of three independent experiments. Asterisks indicate statistically significant differences in the viability of CAMP-treated cells and untreated cells $(* p<0.05$ and $* * p<0.01)$. 


\section{Cell cytotoxicity}

Cell cytotoxicity of the newly designed CAMPs was also determined by MTT cell viability assay (Figure 4-6). Exposure of murine immature dendritic cell line JAWSII (Figure 4-6A) and CHO-K1 (Figure 4-6B) cells to CAMP-t1, CAMP-t2, and CAMP-B at concentrations of $64,128,256$, and $512 \mu \mathrm{g} / \mathrm{ml}$ for 4 to $48 \mathrm{~h}$ did not significantly affect cell viability. However, the viability of both JAWSII and CHO-K1 cells was significantly decreased by treatment with CAMP-A at a concentration equal to or greater than $128 \mu \mathrm{g} / \mathrm{ml}$, indicating a potent toxic sign to host cells in vitro. After treatment with CAMP-A, no significant difference in the percentage of cell viability was observed between $4 \mathrm{~h}$ treatment and $48 \mathrm{~h}$ treatment.

\section{Chemotactic activity}

The chemotactic activity of the CAMPs for JAWSII and CCR2-transfected CHO-K1 cells were determined (Figure 4-7). The results indicated that CAMP-t1 with the N-terminal helix-loop structure of AvBD-12 did not show expected chemotactic activity to either cell line (Figure 4-7A and 7B). Interestingly, CAMP-A with the highest antimicrobial activity showed mild chemotactic activity at a concentration of $64 \mu \mathrm{g} / \mathrm{ml}$ (C.I. $=5.13 ; 77.5 \%$ of wild-type AvBD-12, C.I. = 6.62) for JAWSII cells. As shown in Figure 4-7C, more JAWSII cell migration was induced by CAMP-A with increasing peptide concentrations, ranging from 1 to $64 \mu \mathrm{g} / \mathrm{ml}$. No significant chemotactic activity of CAMP-A for CCR-2 transfected CHO-K1 cells was detected (Figure 4-7B). CAMP-t2 and CAMP-B did not show any chemotactic activity for either JAWSII or CCR2-CHO-K1 cells. 
A

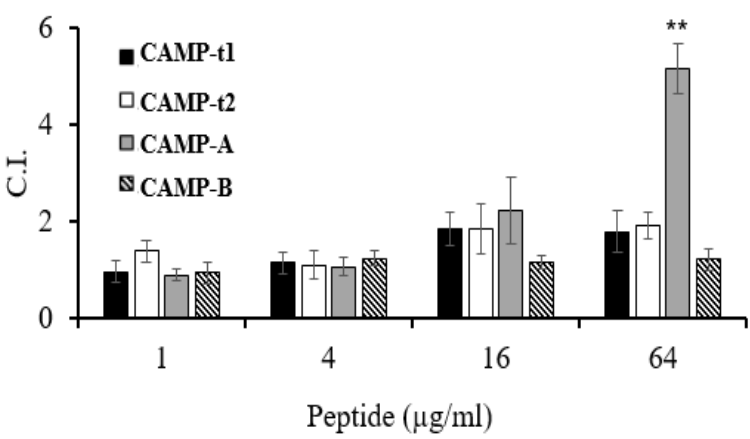

C
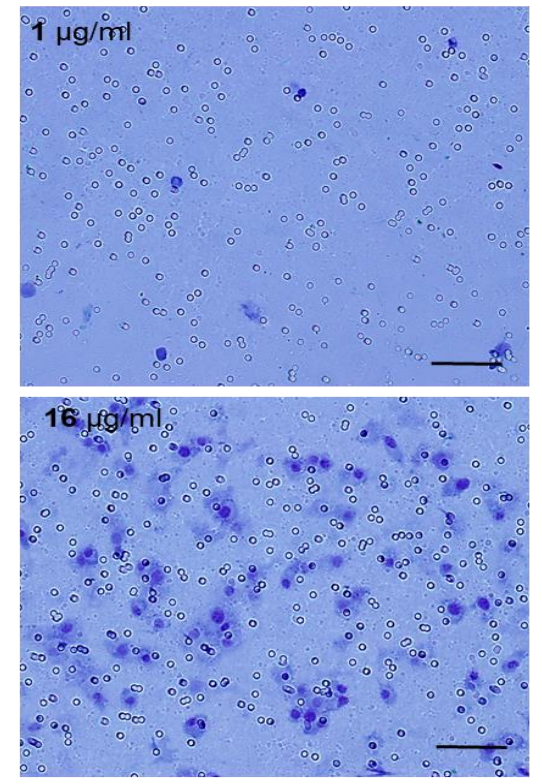

B

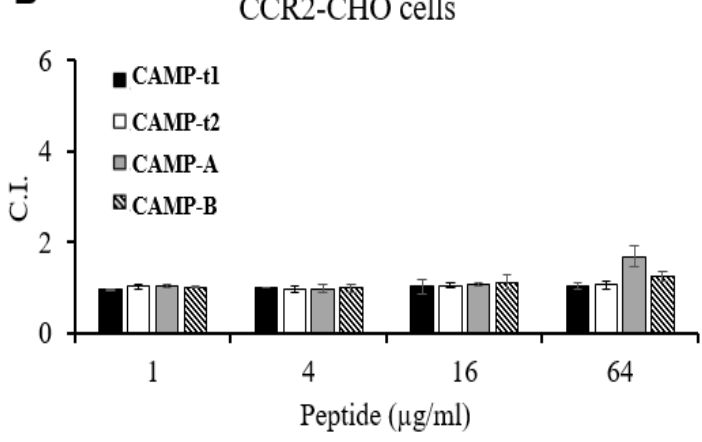

\section{CAMP-A}

\section{Figure 4-7. Chemotactic activity of CAMPs}

CAMP-induced migration of mouse immature dendritic JAWSII cells (A) and CHO-K1 cells expressing avian CCR2 (B) was measured at the following peptide concentrations: 1, 4, 16, and $64 \mu \mathrm{g} / \mathrm{ml}$. (C) Migrated JAWSII cells induced by CAMP-A. Blue ones are stained JAWSII cells. Bar: $50 \mu \mathrm{m}$. Chemotactic index (C.I.) was expressed as the number of migrated cells induced by CAMP / the number of migrated cells in response to chemotactic buffer. Data represent the means $\pm \operatorname{SD}(n=5)$. Asterisks indicate significant difference $(* * p<0.01)$. 


\section{Protease resistance}

The resistance of CAMP-A and CAMP-B, two peptides exhibiting strong antimicrobial activity and salt-resistance, to various proteases was evaluated by subjecting proteasetreated peptides to SDS-PAGE (Figure 4-8). The results indicated that CAMP-A and CAMP-B were partially digested by $\alpha$-chymotrypsin at 0.12 to $1.2 \mu \mathrm{g} / \mathrm{ml}$, as indicated by the presence of peptide bands with lower molecular weight than that of the untreated peptides (Figure 4-8A and 8B). The antimicrobial activity of CAMP-A and CAMP-B against $P$. aeruginosa decreased significantly post-digestion (Figure 4-8C). The digested peptides retained approximately $90 \%$ (at $0.12 \mu \mathrm{g} / \mathrm{ml}$ ), $80 \%$ (at $0.66 \mu \mathrm{g} / \mathrm{ml}$ ), and $30 \%$ (at $1.2 \mu \mathrm{g} / \mathrm{ml})$ of the killing activity of CAMP-A or CAMP-B pre-digestion. In contrast, the anti-Staphylococcus activity was mildly affected only when the CAMPs were treated with the highest concentration $(1.2 \mu \mathrm{g} / \mathrm{ml})$ of $\alpha$-chymotrypsin (Figure $4-8 \mathrm{D})$. The results indicated the digested fragment of CAMP-A and CAMP-B kept high antimicrobial activity against S. aureus. CAMP-A and CAMP-B were not cleaved by metalloproteinases matrilysin, elastase, and cathepsin B at concentrations up to $20 \mu \mathrm{g} / \mathrm{ml}$. Treatment with these proteases did not affect the antimicrobial activity of CAMP-A and CAMP-B (data not shown). 
A

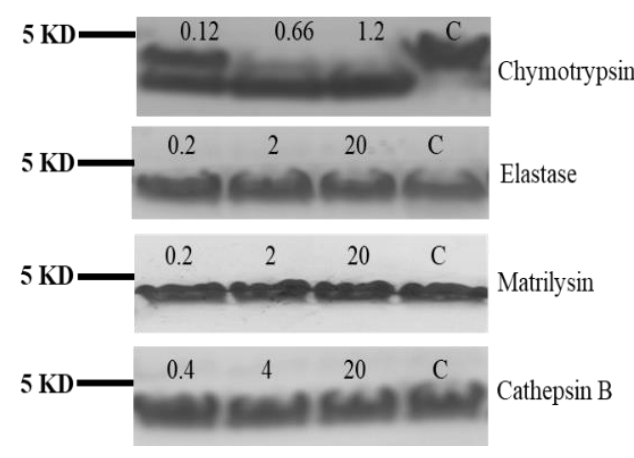

C

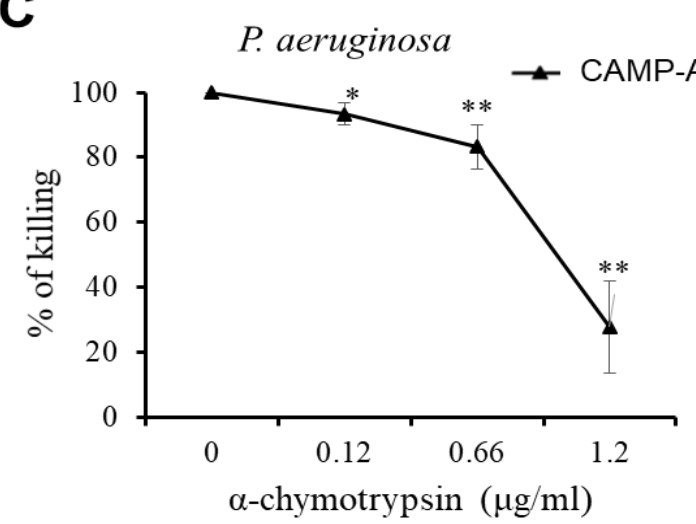

D

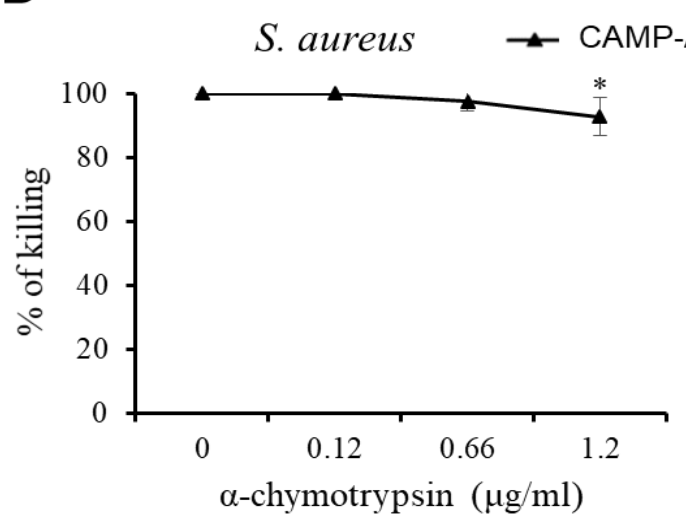

B

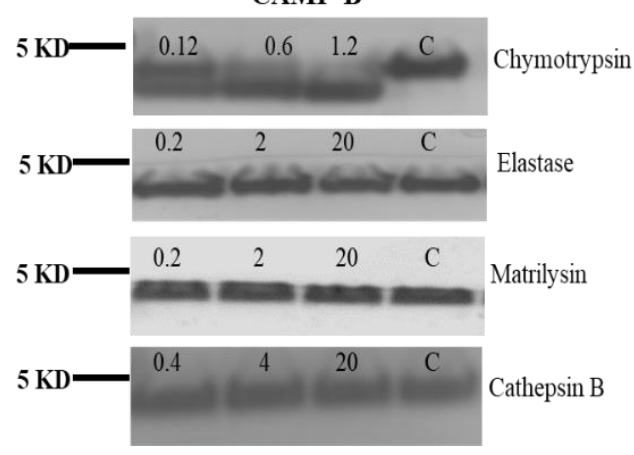

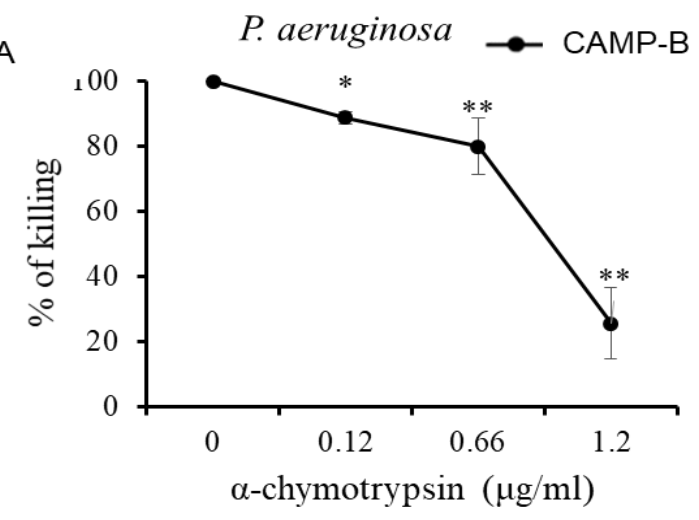

Figure 4-8. Effects of protease treatment on the antimicrobial activity of CAMP-A and CAMP-B against $P$. aeruginosa and S. aureus

Peptides were digested with $\alpha$-chymotrypsin, elastase, matrilysin, or cathepsin B for $1 \mathrm{~h}$ at $37^{\circ} \mathrm{C}$ and subjected to SDS-PAGE (16.5\% polyacrylamide gel) analysis. (A) CAMP-A. 
(B) CAMP-B. (C) Antimicrobial activity of CAMP-A post-digestion by $\alpha$-chymotrypsin at the concentration of $0.12,0.66$, and $1.2 \mu \mathrm{g} / \mathrm{ml}$. (D) Antimicrobial activity of CAMP-B postdigestion by $\alpha$-chymotrypsin at the concentration of $0.12,0.66$, and $1.2 \mu \mathrm{g} / \mathrm{ml}$. Results are expressed as percent killing by digested peptides over untreated peptides. Data are the means $\pm \operatorname{SD}(n=3)$. Asterisks indicate the statistically significant difference between antimicrobial activity with and without protease $\left(* p<0.05\right.$ and $\left.*^{*} p<0.01\right)$.

\section{Discussion}

Host cationic antimicrobial peptides (CAMP) have been broadly investigated due to their broad-spectrum antimicrobial activity and low potential for resistance development compared to conventional antibiotics by attacking multiple unspecific targets. Our previous structure-function analysis of AvBDs and their analogs indicates that the highly concentrated surface positive charge plays a predominant role in the antimicrobial potency of the peptides whereas the CCR2-binding domain (N-terminal $\alpha$-helix along with an adjacent loop) is responsible for the broad-spectrum chemotactic activity for both avian and mammalian dendritic cells [56]. For example, linear AvBD analogs with a high net positive charge (+9), modest hydrophobicity (40\%), and a predicted CCR2 binding domain exhibit strong antimicrobial and mild chemotactic activities. However, the linear peptides designed in our previous study are still lengthy (45 amino acid residues) and susceptible to physiological concentrations of $\mathrm{NaCl}$. Although the sensitivity of AvBD analogs to proteases was not evaluated in our previous studies, investigations conducted by others suggest that linear peptides are susceptible to bacterial metalloprotease, cysteine protease and human neutrophil elastase [38, 39]. To develop antimicrobial peptides suitable for therapeutic or preventive use, we utilized an integrated approach to modify AvBD analogs 
to achieve the following goals: compositionally simple (helix, short and all natural amino acids), resistance to protease digestion and physiological concentrations of cationic salts, non-cytotoxic, strong and broad-spectrum antimicrobial activity and potentially chemotactic for immune cells.

We first designed two CAMP templates, CAMP-t1 and CAMP-t2, by extrapolating the CCR2 binding domain of AvBD-12 (CAMP-t1) and key amino acid residues contributing to the concentrated surface positive charge and hydrophobicity of AvBD-6 (CAMP-t2), respectively. For CAMP-A, the negatively charged amino acid residues (D and E) in AvBD-12 were replaced by positively charged residues ( $K$ and $R$ ). Because the net positive charge of CAMP-t1 was still relatively low (+4), a poly-Trp tail was incorporated into its C-terminus to boost the antimicrobial activity. Trp residues have a strong tendency to insert into membrane lipid bilayer and Trp-rich peptides exhibit enhanced antimicrobial activity and salt resistance [89]. N-terminal acetylation and C-terminal amidation (mimicking native proteins) were also incorporated to increase the metabolic stability of peptides as well as their resistance to enzymatic degradation [96]. To evaluate the antimicrobial properties of these peptides, we determined their MICs against $P$. aeruginosa and Staphylococcus at conditions outlined by CLSI $[117,118]$. Compared to AvBD-6 and AvBD-12 as well as previously designed AvBD analogs, both CAMP-t1 and CAMP-t2 demonstrated improved antimicrobial activity $[56,59]$. Although CAMP-t1 retained the Nterminal $\alpha$-helix and an adjacent loop structure, it lost the desired chemotactic property of AvBD-12 and analogs [56], suggesting that either the amino acid composition was not optimal for CCR2 binding or additional structural components were required for CCR2 binding. The shorter template, CAMP-t2 had stronger antimicrobial activity against 
methicillin-resistant S. pseudintermedius than CAMP-t1 and AvBD-6 but was sensitive to high concentrations of cationic salts. The study was then focused on improving the antimicrobial property and reducing the salt-sensitivity of CAMP-t 2 which adopted a coil structure in lipid condition.

Studies on the mechanism of action of CAMPs have indicated that amphipathicity is a key characteristic required for membrane permeabilization in which hydrophobic residues interact with membrane lipid components while hydrophilic regions either bind with the phospholipid head groups or form the lumen of a membrane pore [59, 163]. Alpha-helical peptides with hydrophobic and hydrophilic residues on opposite sides of the peptide molecule confer antimicrobial property [17]. The shorter template, CAMP-t2 with coilhelix structure (35\% of a.a. residues adopted $\alpha$-helical structure), was further modified to form an $\alpha$-helical structure which confers structural stability [94]. To maximize the antimicrobial activity and minimize the damaging effect on host cell membrane, Trp and Pro residues were incorporated and the amino acid residues were strategically arranged to avoid protease cutting sites predicted using online PROSPER and SignalP 4.1 servers. The resulting peptides, CAMP-A and CAMP-B, demonstrated strong antimicrobial activity against bacterial reference strains as well as multi-drug resistant $P$. aeruginosa and methicillin-resistant $S$. pseudintermedius clinical isolates. With a poly-Trp tail, $\alpha$-helical structure and increased surface positive charge, these peptides were fully functional at physiological concentrations of $\mathrm{NaCl}$ and $\mathrm{CaCl}_{2}$. These CAMPs were also resistant to metalloproteinases, matrilysin and elastase, and cathepsin B at concentrations higher than that in bacterial protein secretion [182] or in mammalian host cells [178]. Although they were still cleaved by $\alpha$-chymotrypsin, the antimicrobial activity was minimally affected at 
the concentration of $0.12 \mu \mathrm{g} / \mathrm{ml}$, about 3 to 40 times higher than the concentration tested in human by different methods, $4 \mathrm{ng} / \mathrm{ml}$ [183] and $37.5 \mathrm{ng} / \mathrm{ml}$ [184], respectively. At a high concentration $(1.2 \mu \mathrm{g} / \mathrm{ml})$, $\alpha$-chymotrypsin treatment significantly reduced the killing activity against $P$. aeruginosa $(p<0.05)$ but not $S$. aureus $(p>0.05)$. The discrepancy could be associated with compositional or structural differences between the membranes of Gram-negative and Gram-positive bacteria which warrants further investigation into the mechanism of antimicrobial action of these peptides.

Our previous studies have shown that AvBDs could disrupt bacterial membrane resulting in cell deformation, increased membrane permeabilization, and membrane damage $[56,59]$. In the present study, CAMP-treated $P$. aeruginosa and $S$. aureus were stained by propidium iodide (PI) indicating that the primary mode of action utilized by these CAMPs was membrane attacking, which is considered a mechanism less likely to trigger bacterial resistance. Although the CAMPs under investigation had strong antimicrobial activity, they did not show any detectable cytotoxicity and hemolytic activity at the doses required for effective bacterial killing. CAMP-t1, CAMP-t2, and CAMP-B had minimal cytotoxic and hemolytic activities at a relatively high peptide concentration $(512 \mu \mathrm{g} / \mathrm{ml})$. CAMP-A, the most potent antimicrobial peptide, exhibited hemolytic and cytotoxic activities at concentrations equal to or greater than $128 \mu \mathrm{g} / \mathrm{ml}$ which, however, was 6-fold higher than the MIC against $P$. aeruginosa and 4-fold higher than the MIC against Staphylococcus spp. The undesired hemolytic activity was mild compared to other antimicrobial peptides including magainin isolated from the skin of African frog Xenopus laevis and melittin from bee venom [185]. The cytotoxic property of CAMP-A was not surprising because the peptide had a relatively high hydrophobicity (50\%), which is known hydrophobicity is 
associated with peptide cytotoxic effect [186].

It has been suggested that the three conserved disulfide bonds were required for the chemotactic function of $\beta$-defensins [59, 121, 127, 187]. Data from our previous study indicated that a predicted CCR2 binding domain ( $\mathrm{N}$-terminal $\alpha$-helix and an adjacent $\beta 2$ $\beta 3$ loop) in AvBD-12A3 (a linear peptide) without disulfide bonds was chemotactic to JAWSII cells [56]. In the present study, CAMP-t1 with a similar helix-loop domain had no chemotactic activity. Interestingly, CAMP-A with high positive charge and modest hydrophobicity induced chemotactic migration of JAWSII cells which occurred possibly via formyl-peptide receptors like helical CAMPs that were reported for human cathelicidin LL-37 [188] and cathelicidin-like pleurocidins [189].

\section{Conclusions}

Multiple favorable attributes can be integrated into short CAMPs for the development of antimicrobial chemotherapeutic agents. CAMP-t1 with a predicted CCR binding domain of AvBD-12 demonstrated improved antimicrobial activity but lost the original chemotactic function. CAMP-t2 with key amino acid residues of AvBD-6 also showed strong antimicrobial activity and sensitivity to high concentrations of cationic salts. Modification of CAMP-t2 by increasing hydrophobicity and addition of Trp and/or Pro residues further improved the antimicrobial activity and resistance to salts and proteases. CAMP-A and CAMP-B with potent antimicrobial activity against multidrug-resistant $P$. aeruginosa and methicillin-resistant Staphylococcus, rapid membrane attacking action, resistance to salts and proteases, and moderate therapeutic index are excellent candidates for the development of antimicrobial agents. 


\title{
CHAPTER 5: Antimicrobial Efficacy of Selected CAMPs against $P$. aeruginosa Infection in a Murine Skin Wound Infection Model
}

\begin{abstract}
Pseudomonas aeruginosa is one of the common pathogens in wound infections. The treatment for $P$. aeruginosa infection is challenging due to its natural resistance to conventional antibiotics. Cationic antimicrobial peptides (CAMPs) have antibacterial activity with different modes of action with antibiotics, which are promising therapeutic agents. In the present study, we evaluated the safety and efficacy of CAMP-A and CAMPB, two antimicrobial peptides derived from AvBD-6, for the treatment of P. aeruginosa infection in murine surgical wounds. Complete blood counts, blood chemistry, and histological examinations revealed that CAMP-A and CAMP-B were nontoxic at a dose of 4×MIC. However, high doses of CAMP-A $(5 \times \mathrm{MIC}$ or $50 \times \mathrm{MIC})$ caused an increase in ALT level in 1 of 7 mice. The efficacy study was carried out in two trials. In the first trial, at low peptide concentration $(2 \times \mathrm{MIC})$, both CAMP-A and CAMP-B significantly inhibited the bacterial proliferation in skin wound in a time-dependent manner. CAMP-A exhibited stronger antimicrobial activity than CAMP-B, and further reduced viable bacterial counts from $5.8 \log _{10} \mathrm{CFU} / \mathrm{g}$ skin to $4.3 \log _{10} \mathrm{CFU} / \mathrm{g}$ skin and prevented infection in the liver and kidneys at day 5 post-infection. In the second trial, the killing efficacy of CAMP-A at higher doses of $(5 \times \mathrm{MIC}$ and $50 \times \mathrm{MIC})$ was further evaluated. The results showed that the antimicrobial efficacy of CAMP-A was dose-dependent. Treatment with 50×MIC of CAMP-A caused the viable bacterial counts of skin wound down to $3.8 \log _{10} \mathrm{CFU} / \mathrm{g}$ skin. Application of CAMP-A at both concentrations prevented bacterial infection in the liver and kidneys. In conclusion, the results suggest that CAMP-A has strong killing efficacy
\end{abstract}


against $P$. aeruginosa infection in skin wound and can prevent systemic infection, which is a potential therapeutic agent.

\section{Background}

P. aeruginosa is the leading cause of secondary infections in immunocompromised hosts, especially patients with skin wounds [190]. According to 2015 CDC Report (http://www.cdc.gov/hai/organisms/pseudomonas.html), above 13\% (6,000 cases) of 51,000 cases of $P$. aeruginosa infections were caused by multidrug-resistant strains each year in the United States, resulting in more than 400 deaths. P. aeruginosa infections are associated with a poor prognosis due to its natural resistance to many antibiotics $[191,192]$. P. aeruginosa colonization of severe wounds and its rapid proliferation within the damaged tissues often lead to disseminated infections, resulting in bacteremia and septic shock [193]. Currently, beta-lactam antibiotics alone or in combination with aminoglycosides are the primary therapeutic agents against $P$. aeruginosa infections. However, resistance to betalactam antibiotics, aminoglycosides, and quinolones is increasing rapidly [194, 195]. The emergence of multidrug-resistant bacteria reduces the efficacy of existing antibiotics [196]. Host cationic antimicrobial peptides (CAMPs) constituting the first line of innate defense against microbial pathogens [163] have been considered as new classes of antimicrobial agents. These peptides have different modes of action involving nonspecific membranedisruption and targeting intracellular components, which makes it difficult for microbes to develop resistance [162]. Therefore, researchers have begun to evaluate host AMPs and their analogs as new therapeutic agents $[164,197,198]$. To date, only about ten peptide products are under clinical trials and several others are at the preclinical stage (mainly for topical use) [164]. 
Avian $\beta$-defensins (AvBDs) have pleiotropic biological functions, such as broad-spectrum antimicrobial activity, LPS-neutralizing ability, and chemotactic activity [56, 57, 59]. To simplify the structure of AvBDs, CAMP-t1 and CAMP-t2 were designed with the key domains and residues for chemotactic and antimicrobial properties of AvBD-12 and AvBD-6, respectively [56]. An integrated approach involving the optimization of net positive charge, amphipathicity, and $\alpha$-helical structure of CAMP-t2 was utilized to design short and compositionally simple peptides (CAMP-A and CAMP-B) with potent antimicrobial activity, improved resistance to salts and proteases, and minimal cytotoxicity to host cells. In vitro antimicrobial results indicated that CAMP-A and CAMP-B had significantly improved antimicrobial activity against $P$. aeruginosa and Staphylococcus spp., including clinical isolates of multidrug-resistant $P$. aeruginosa and methicillinresistant S. pseudintermedius (MRSP) at physiological concentrations of salts and proteases (Chapter 4).

In the present study, in vivo efficacy of CAMP-A and CAMP-B against $P$. aeruginosa infection was investigated using a mouse skin wound model. Prior to treatment, bacterial dose and time needed to disseminate from wound to systemic organs, and the acute toxicity of CAMPs were determined. The efficacy study was carried out in two clinical trials.

\section{Materials and Methods}

\section{Bacterial culture}

Pseudomonas aeruginosa (P. aeruginosa, ATCC 27853) was maintained and cultured in Luria-Bertani broth or agar (LB, BD Difco ${ }^{\mathrm{TM}}$ ) at $37^{\circ} \mathrm{C}$ as described previously [56].

\section{Peptides}

The design of CAMP-A and CAMP-B were based on the key residues of AvBD-6, as 
described in Chapter 4. All peptides were custom synthesized using the standard solid phase 9-fluorenylmethoxycarbonyl (Fmoc) method and purified by reverse phase highperformance liquid chromatography (RP-HPLC) (Lifetein, NJ) [59]. The purity of the synthetic CAMPs was greater than $98.5 \%$ as verified by liquid chromatography-mass spectrometry (LC-MS) (Lifetein, NJ).

\section{Animal source and care}

Specific-pathogen-free C57BL/6 mice, female, 6 weeks of age, were purchased from The Jackson Laboratory (Bar Harbor, MN). Mice were raised one week in filter-top static cages in an Association for Assessment and Accreditation of Laboratory Animal Care International (AALAC)-accredited facility for acclimation before experiments, with ad libitum water and 5008 Formulab Diet (LabDiet, St. Louis, MO), in conditions of a relative humidity between $30-70 \%$, temperature of $20-26^{\circ} \mathrm{C}$, and a $12: 12 \mathrm{~h}$ light: dark cycle. All procedures were performed in accordance with University of Missouri Institutional Animal Care and Use guidelines [199].

\section{Establishment of skin wound model}

The skin wound model was established according to a previously published method with minor modifications [200, 201]. Briefly, mice were weighed and anesthetized with an intraperitoneal injection of ketamine, $90 \mathrm{mg} / \mathrm{kg}$ (KetaVed, Vedco, St. Joseph, MO) and xylazine, 10 mg/kg (X-Ject E Injection, Henry Schein Animal Health, Dublin, OH). Mice were prepared for surgery by lubricating eyes, then clipping hair from the dorsal thorax through the neck region followed by aseptic scrub three times each with povidone-iodine followed by $70 \%$ ethanol. After anesthetized, a $4 \mathrm{~mm}$ diameter full-thickness wound (the epidermis and dermis layers) was cut using a sharp curved surgical scissor in this area. A 
steam-sterilized black rubber washer with a $6 \mathrm{~mm}$ inner diameter bore was carefully stuck around the skin defect with Cyanoacrylate adhesive (Zoetis, Parsippany, NJ), not disturbing the wound. The rubber was further fixed with black \#4-0 nylon suture (Monosof $^{\mathrm{TM}}$, Medtronic, Minneapolis, MN) through each quadrant of the washer and the surrounding skin, forming a splint to prevent healing by contraction. A piece of non-latex, hypoallergenic adhesive (Tegaderm Transparent Dressing, 3M Animal Care Products, St. Paul, MN) was placed over the defect, completely covering while being trimmed to the edge of the rubber splint. The mice were given $0.05 \mathrm{mg} / \mathrm{kg}$ buprenorphine (Buprenex, Reckitt Benckiser Pharmaceuticals Inc., Richmond, VA) and anesthetic reversal with 1 $\mathrm{mg} / \mathrm{kg}$ tolazoline (Tolazil, VET ONE, Boise, ID), both diluted in 0.9\% NaCl (Hospira, Inc., Lake Forest, IL). Mice recovered in sterile filter-top covered cages under clean conditions. Establishment of $P$. aeruginosa infection

The surgical wound was inoculated with about $2.5 \times 10^{6} \mathrm{CFU}$ of $P$. aeruginosa in sterile saline before covering the rubber washer. Three groups of mice (4 mice/time point) were sacrificed at 4,24 , and $48 \mathrm{~h}$ post-infection. The skin of wounded area, liver, and kidneys were excised and homogenized. Serial dilution of tissue homogenates were cultured on LB agar plates overnight at $37^{\circ} \mathrm{C}$ and the colony forming units (CFU) were recorded to determine bacterial load in the wound tissue and systemic organs [201].

\section{The acute toxicity of CAMPs}

The first toxicity study included 3 groups of mice ( 7 mice per group). Mice in the control group received a daily dose of $50 \mu \mathrm{l}$ of sterile $0.9 \% \mathrm{NaCl}$ for 7 days. Mice in the two treatment groups received $50 \mu 1$ of CAMP-A and CAMP-B via topical application on the wound at doses of 64 and $128 \mu \mathrm{g} / \mathrm{ml}$ (equivalent to $4 \times \mathrm{MIC}$ ), respectively. The second 
toxicity study also included three groups of mice ( 7 mice per group). Mice in the control group received a daily dose of $50 \mu \mathrm{l}$ of sterile $0.9 \% \mathrm{NaCl}$ for 7 days. Mice in the two treatment groups received $50 \mu \mathrm{l}$ of CAMP-A at $80 \mu \mathrm{g} / \mathrm{ml}(5 \times \mathrm{MIC})$ and $800 \mu \mathrm{g} / \mathrm{ml}$ (50×MIC), respectively. Following application of $0.9 \% \mathrm{NaCl}$ or CAMPs, mice were observed daily for 7 days for any signs of toxic effect. Changes in behavior, body weight, and smoothness of skin hair were scored using the following criteria: no change in activity, body weight, and smooth and bright hair coat = no signs (0); quiet but active when touched, mildly scruffy hair coat, or up to $10 \%$ weight loss = minor signs $(0.2)$; poor motility, moderately scruffy hair coat, or $10-20 \%$ of weight loss = mild signs (0.6); poor motility even under stimulation, very wiry hair coat, or $>20 \%$ of weight loss $=$ manifest signs $(0.8)$. According to the animal use protocol, veterinary care would have been provided to animal scored $\geq 0.8$ and moribund animals euthanized to avoid unnecessary suffering. At the end of study (day 7), blood was collected by cardiac puncture from each mouse into a Lithium Heparin tube (Greiner Bio-One) for laboratory analysis. Mice were sacrificed and wounded skin, livers, and kidneys were collected for pathological examinations.

\section{Hematological and biochemical analysis}

Hematological analysis was performed using the Sysmex XT2000i V Automated Hematology Analyzer (Sysmex America Inc., IL). The number of white blood cells (WBC), red blood cells (RBC), hemoglobin (HGB), hematocrit (HCT), platelet count (PLT), neutrophils (NEUT), lymphocytes (LYMP), monocytes (MONO), eosinophils (EOSI), basophils (BASO), and red cell indices: mean cell volume (MCV), mean cell hemoglobin $(\mathrm{MCH})$, mean cell hemoglobin concentration $(\mathrm{MCHC})$, and red cell distribution width (RDW), were counted. Blood biochemical profile analysis was carried out using the 
Olympus 400AUe Chemistry Analyzer (Olympus Corporation, PA). Alkaline aminotransferase (ALT), albumin (ALB), and gamma-glutamyltransferase (GGT) were used to assess liver and kidney functions.

\section{Histopathological evaluations}

At necropsy, systemic organs were examined for gross lesions. Specimens of wounded skin, liver, and kidneys of each mouse were excised and immediately fixed in $10 \%$ of formalin for 2 days. The fixed tissues were embedded in paraffin, sectioned at $5 \mu \mathrm{m}$ thickness, and stained with hematoxylin and eosin $(\mathrm{H} \& \mathrm{E})$. Three sections of each tissue were evaluated and scored by a pathologist unaware of the treatment conditions. The following scale was used to score inflammation in skin, liver, and kidneys: $0=$ none (no inflammation); $1=$ minimal inflammation involving $<5 \%$ of tissue specimen; $2=$ moderate with focally extensive areas of inflammation ( $5 \%$ to $25 \%$ of tissue, e.g. involving epidermis and dermis); $3=$ moderate to severe with focally extensive areas of inflammation and fibrosis $(>25 \%$ to $50 \%$ of tissue, e.g. involving dermis, epidermis and subcutis).

\section{The treatment efficacy of CAMPs}

In the first trial, three individual experiments were conducted for 3 treatment durations: 1 day, 3 days and 5 days. Each experiment included 4 treatment groups (6 mice per group): 2×MIC CAMP-A, 2×MIC CAMP-B, polysporin, and $0.9 \% \mathrm{NaCl}$. The second trial included 4 treatment groups: 5×MIC CAMP-A, 50×MIC CAMP-A, polysporin, and $0.9 \% \mathrm{NaCl}$, and the treatment continued for 5 days. Polysporin contains two active ingredients including 500 units of bacitracin and 10, 000 units of polymyxin B in each gram.

On the surgical day, skin wound was created and inoculated with approximately $2.5 \times 10^{6}$ CFU of $P$. aeruginosa. At 4-h post inoculation, $50 \mu 1$ of peptide, polysporin, or $0.9 \% \mathrm{NaCl}$ 
were applied to the wounds of mice in appropriate groups. The body weight, wound healing status, and behavior of mice were recorded daily. When the treatment ended, mice were sacrificed. The skin of the wound area, liver, and kidneys of each mouse were collected and processed separately laboratory evaluations. A portion of the tissue was homogenized for bacterial culture, and another portion was fixed in $10 \%$ formalin for histopathological evaluation. The treatment efficacy was determined based on wound healing, degree of inflammation, and bacterial burden in local and systemic organs.

\section{Statistical analysis}

Data presented were the means \pm standard deviation $(\mathrm{SD})$. Differences between groups were analyzed using the one-way analysis of variance (ANOVA) followed by Duncan's test for multiple comparisons using software SPSS version 19.0 (IBM Corp., Armonk, NY). Differences at $p<0.05$ level among different groups were considered statistically significant.

\section{Results}

\section{Establishment of $P$. aeruginosa infection}

Following inoculation of the wound with $2.5 \times 10^{6} \mathrm{CFU}$ of bacteria, the average CFU count in the wounded skin was 7.0 $\log _{10} \mathrm{CFU} / \mathrm{g}$ at $4 \mathrm{~h}, 7.67 \log _{10} \mathrm{CFU} / \mathrm{g}$ at $24 \mathrm{~h}$, and $8.29 \log _{10}$ CFU/g at $48 \mathrm{~h}$ (Figure 5-1A). P. aeruginosa was first detected in the liver of a mouse at 24 $\mathrm{h}$ (Figure 5-1B), and the bacteria spread to both the liver and kidneys at $48 \mathrm{~h}$ post inoculation (Figure 5-1B and 1C). The CFU counts of $P$. aeruginosa in the infected liver and kidneys were around $4 \log _{10} \mathrm{CFU} / \mathrm{g}$ at $48 \mathrm{~h}$. The bacteria recovered from mice were confirmed to be $P$. aeruginosa using a matrix-assisted laser desorption/ionization-time of flight mass spectrometry (MALDI-TOF-MS) [202]. 


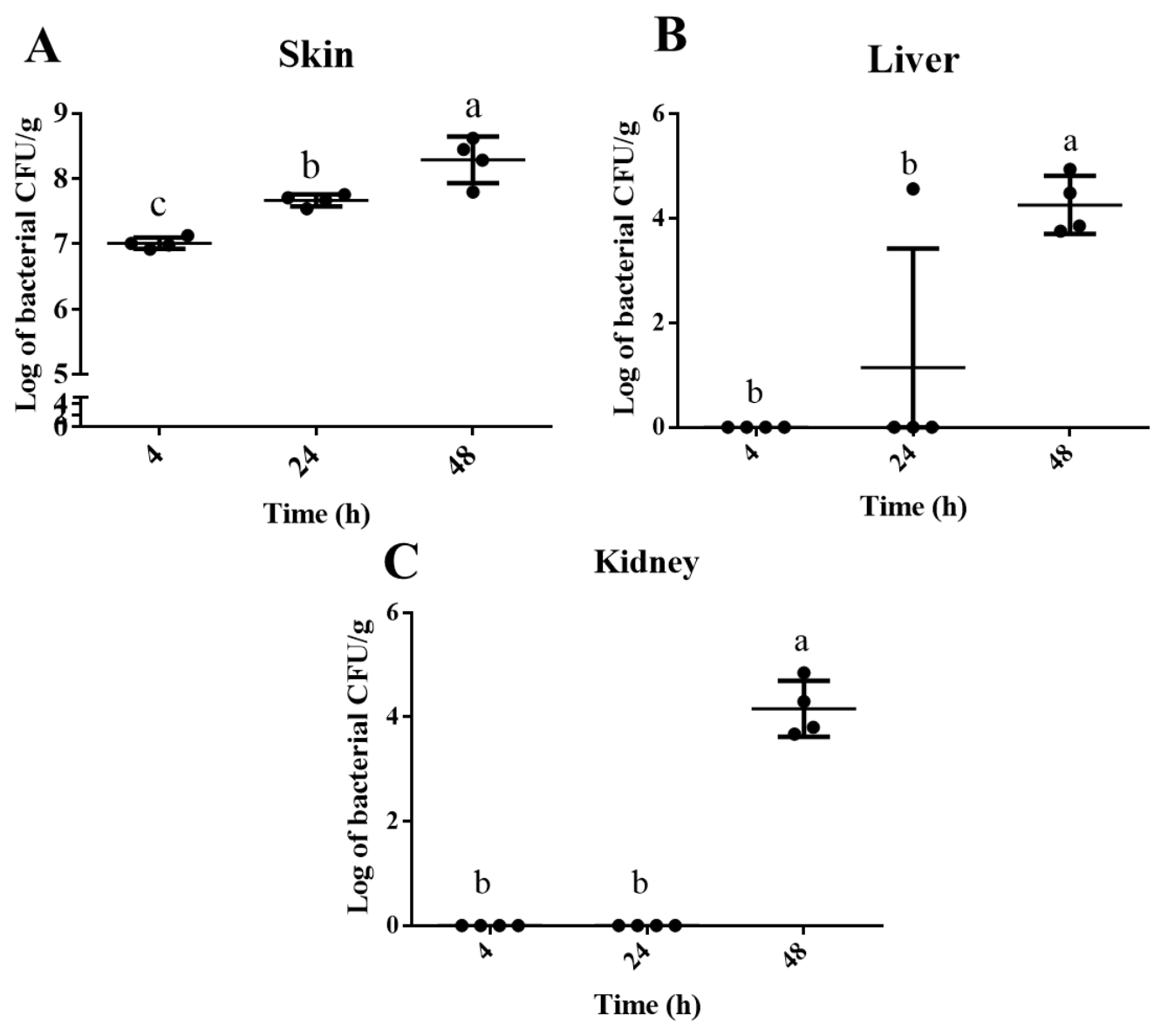

Figure 5-1. Establishment of $P$. aeruginosa infection in mice

$2.5 \times 10^{6} \mathrm{CFU}$ of bacteria were inoculated into the skin wound. The mice were sacrificed at 4,24 , and $48 \mathrm{~h}$ post-infection. Serial dilutions of tissue homogenates were cultured on LB agar plates and the bacterial colony forming units $(\mathrm{CFU})$ were enumerated on the following day, and $\log _{10} \mathrm{CFU} / \mathrm{gram}$ tissue was calculated. Data are presented as average \pm standard deviations ( $\mathrm{SD}, \mathrm{n}=4)$. Different letters indicate significant differences among different time points $(p<0.05)$. 


\section{The acute toxicity of CAMP-A and CAMP-B}

In trial 1, CAMP-A and CAMP-B were applied daily to the skin wound at doses equivalent to $4 \times$ MIC for 7 days. No significant differences in body weight, physical activity, and smoothness of skin hair were observed among treatment groups (Figure 5-2A and 2B). No significant differences in the hematological and blood biochemical parameters, as well as enzymes (ALT, GGT, and ALB) were detected among groups (Table 5-1 and 5-2). In trial 2, treatments of mice with higher doses of CAMP-A was associated with elevated ALT level. The average ATL levels of mice treated with 5×MIC CAMP-A and 50×MIC CAMPA were 148.3 $\pm 99.9 \mathrm{U} / \mathrm{L}$ and 103.2 $\pm 157.3 \mathrm{U} / \mathrm{L}$, respectively, compared 49.0 $\pm 28.7 \mathrm{U} / \mathrm{L}$ seen in the control group (Table 5-2). The elevated average ALT value was due to a significant rise of ALT in one mouse (312 U/L) in the 5×MIC CAMP-A treatment group (14\% of mice) and one mouse $(384 \mathrm{U} / \mathrm{L})$ in the $50 \times$ MIC CAMP-A treatment group (14\% of mice). However, partial hemolysis was observed in both samples which might have skewed the ATL values. 

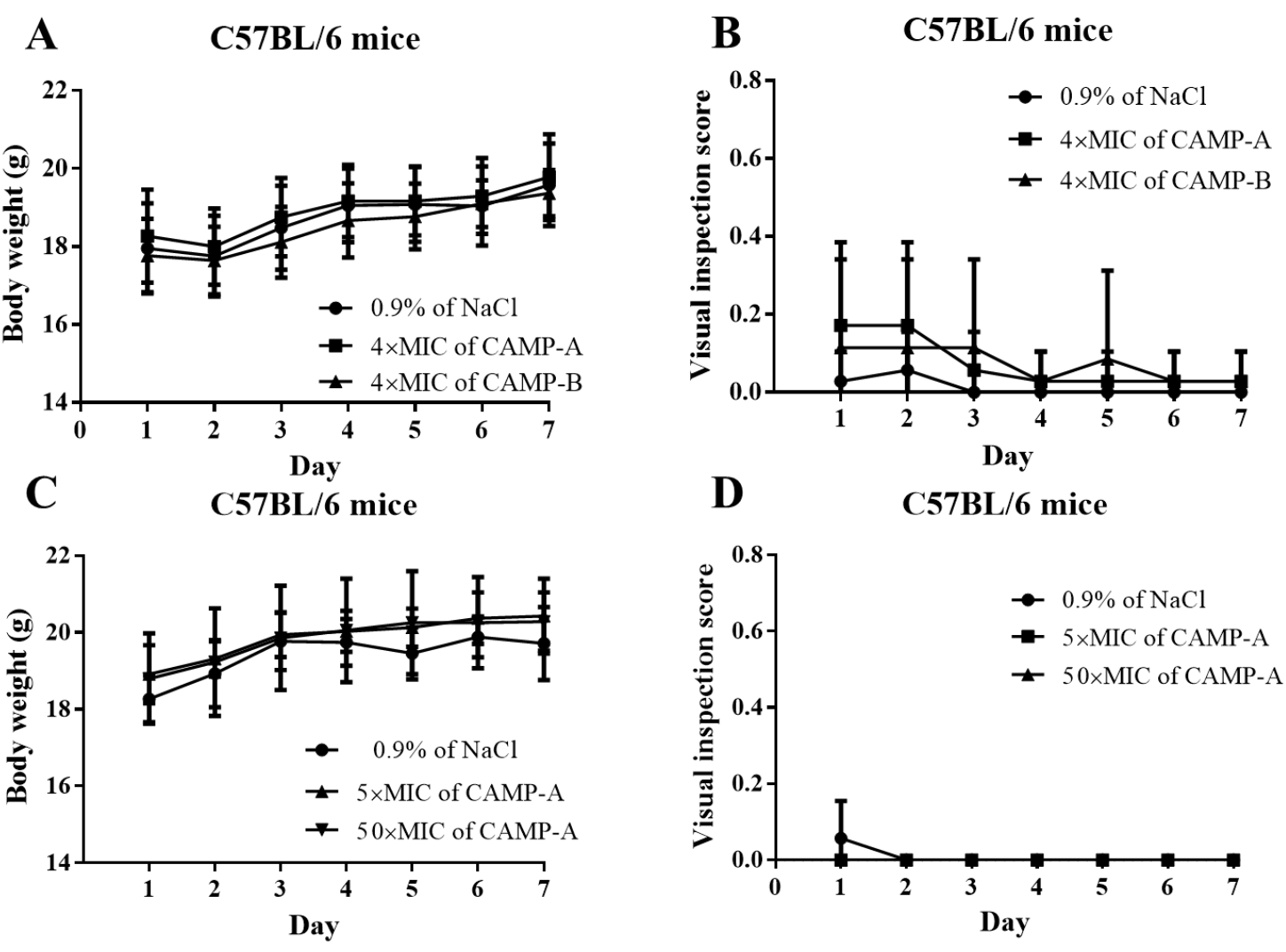

Figure 5-2. Effect of CAMPs on body weight and visual inspection scores

Following treatment, body weight, physical activities, and smoothness of skin hair were evaluated daily for 7 days. (A) and (B), changes in body weight visual inspection scores, respectively, after treatment with $4 \times$ MIC CAMP-A and CAMP-B. (C) and (D), changes in body weight and visual inspection scores, respectively, after treatment with $5 \times$ and $50 \times$ MIC CAMP-A. No significant difference was observed among treatment groups in each trial. 
Table 5-1. Effect of low doses $(4 \times M I C)$ of CAMP-A and CAMP-B on the hematological and blood biochemical parameters

\begin{tabular}{|c|c|c|c|c|c|c|c|c|}
\hline \multirow{2}{*}{$\begin{array}{r}\text { Category } \\
\text { Hematology } \\
\end{array}$} & \multicolumn{2}{|c|}{ Reference } & \multicolumn{2}{|c|}{ Control } & \multicolumn{2}{|c|}{ CAMP-A (4×MIC) } & \multicolumn{2}{|c|}{ CAMP-B (4×MIC) } \\
\hline & Range & Unit & Average & STDEV & Average & STDEV & Average & STDEV \\
\hline WBC & $0.80-10.60$ & $10^{\wedge} 3 / \mu \mathrm{L}$ & 6.664 & 1.140 & 7.174 & 2.609 & 6.081 & 1.161 \\
\hline $\mathrm{RBC}$ & $6.50-11.50$ & $10^{\wedge} 6 / \mu \mathrm{L}$ & 9.350 & 0.345 & 9.503 & 0.416 & 8.979 & 1.026 \\
\hline HGB & $11.0-16.5$ & $\mathrm{~g} / \mathrm{dL}$ & 13.980 & 0.349 & 14.271 & 0.531 & 14.029 & 1.005 \\
\hline HCT & $35.0-55.0$ & $\%$ & 44.200 & 1.643 & 45.143 & 1.952 & 43.143 & 3.132 \\
\hline $\mathrm{MCV}$ & $41.0-55.0$ & $\mathrm{fL}$ & 47.200 & 0.837 & 47.286 & 0.756 & 48.286 & 2.984 \\
\hline $\mathrm{MCH}$ & $13.0-18.0$ & pg & 15.000 & 0.485 & 15.043 & 0.282 & 15.729 & 0.921 \\
\hline $\mathrm{MCHC}$ & $30.0-36.0$ & $\mathrm{~g} / \mathrm{dL}$ & 31.840 & 1.163 & 31.700 & 0.678 & 32.571 & 0.854 \\
\hline Platelet Count & $400-1600$ & $10^{\wedge} 3 / \mu \mathrm{L}$ & 1021.400 & 155.307 & 937.714 & 251.844 & 965.714 & 122.545 \\
\hline Neutrophil & $0.23-3.60$ & $10^{\wedge} 3 / \mu \mathrm{L}$ & 1.618 & 0.437 & 1.433 & 0.708 & 1.380 & 0.621 \\
\hline Lymphocyte & $0.60-8.90$ & $10^{\wedge} 3 / \mu \mathrm{L}$ & 4.568 & 1.133 & 5.042 & 2.140 & 4.041 & 0.805 \\
\hline Monocyte & $0.04-1.40$ & $10^{\wedge} 3 / \mu \mathrm{L}$ & 0.220 & 0.189 & 0.191 & 0.087 & 0.244 & 0.168 \\
\hline Eosinophil & $0.00-0.51$ & $10^{\wedge} 3 / \mu \mathrm{L}$ & 0.220 & 0.068 & 0.459 & 0.421 & 0.373 & 0.220 \\
\hline Basophil & $0.00-0.12$ & $10^{\wedge} 3 / \mu \mathrm{L}$ & 0.038 & 0.019 & 0.063 & 0.059 & 0.030 & 0.017 \\
\hline Neutrophil & $6.5-50.0$ & $\%$ & $24.60 \%$ & 0.08 & $21.71 \%$ & 0.11 & $22.71 \%$ & 0.10 \\
\hline Lymphocyte & $40.0-92.0$ & $\%$ & $68.00 \%$ & 0.07 & $69.29 \%$ & 0.09 & $66.71 \%$ & 0.07 \\
\hline Monocyte & $0.9-18.0$ & $\%$ & $3.20 \%$ & 0.03 & $2.71 \%$ & 0.01 & $3.86 \%$ & 0.02 \\
\hline Eosinophil & $0.0-7.5$ & $\%$ & $3.40 \%$ & 0.01 & $5.57 \%$ & 0.03 & $6.00 \%$ & 0.03 \\
\hline Basophil & $0.0-1.5$ & $\%$ & $0.72 \%$ & 0.004 & $0.73 \%$ & 0.004 & $0.67 \%$ & 0.004 \\
\hline \multicolumn{9}{|l|}{ Biochemistry } \\
\hline ALT & $27-195$ & $\mathrm{U} / \mathrm{L}$ & 46.400 & 41.150 & 27.167 & 8.704 & 75.500 & 45.713 \\
\hline ALB & $2.4-4.3$ & $\mathrm{~g} / \mathrm{dL}$ & 2.960 & 0.114 & 2.929 & 0.160 & 2.840 & 0.152 \\
\hline GGT & $0-9$ & $\mathrm{U} / \mathrm{L}$ & $<3$ & 0.000 & $<3$ & 0.000 & $<3$ & 0.000 \\
\hline
\end{tabular}

WBC: white blood cells; RBC: red blood cells; HGB: hemoglobin; HCT: hematocrit; MCV: mean cell volume; $\mathrm{MCH}$ : mean cell hemoglobin; $\mathrm{MCHC}$ : mean cell hemoglobin; RDW: red cell distribution width; ALT: alanine transferase; ALB: albumin; GGT: gammaglutamyltransferase. 
Table 5-2. Effect of high doses (5xMIC and $50 \times$ MIC) of CAMP-A on the hematological and blood biochemical parameters

\begin{tabular}{|c|c|c|c|c|c|c|c|c|}
\hline \multirow{2}{*}{$\begin{array}{c}\text { Category } \\
\text { Hematology }\end{array}$} & \multicolumn{2}{|c|}{ Reference } & \multicolumn{2}{|c|}{ Control } & \multicolumn{2}{|c|}{ CAMP-A (5×MIC) } & \multicolumn{2}{|c|}{ CAMP-A $(50 \times M I C)$} \\
\hline & Range & Unit & Average & STDEV & Average & STDEV & Average & STDEV \\
\hline WBC & $0.80-10.60$ & $10^{\wedge} 3 / \mu \mathrm{L}$ & 4.93 & 1.27 & 5.86 & 0.71 & 5.29 & 0.78 \\
\hline $\mathrm{RBC}$ & $6.50-11.50$ & $10^{\wedge} 6 / \mu \mathrm{L}$ & 8.96 & 0.52 & 8.69 & 0.31 & 8.74 & 0.24 \\
\hline HGB & $11.0-16.5$ & $\mathrm{~g} / \mathrm{dL}$ & 14.84 & 0.73 & 14.53 & 0.25 & 14.50 & 0.33 \\
\hline HCT & $35.0-55.0$ & $\%$ & 42.31 & 2.11 & 41.25 & 1.07 & 41.32 & 1.24 \\
\hline $\mathrm{MCV}$ & $41.0-55.0$ & $\mathrm{fL}$ & 47.26 & 0.49 & 47.53 & 0.68 & 47.30 & 0.14 \\
\hline $\mathrm{MCH}$ & $13.0-18.0$ & pg & 16.59 & 0.28 & 16.70 & 0.38 & 16.60 & 0.13 \\
\hline $\mathrm{MCHC}$ & $30.0-36.0$ & $\mathrm{~g} / \mathrm{dL}$ & 35.09 & 0.48 & 35.18 & 0.53 & 35.15 & 0.34 \\
\hline Platelet Count & $400-1600$ & $10^{\wedge} 3 / \mu \mathrm{L}$ & 754.50 & 157.60 & 805.75 & 98.96 & 873.40 & 62.92 \\
\hline Neutrophil & $0.23-3.60$ & $10^{\wedge} 3 / \mu \mathrm{L}(\%)$ & 0.72 & 0.30 & 0.92 & 0.10 & 0.86 & 0.28 \\
\hline Lymphocyte & $0.60-8.90$ & $10^{\wedge} 3 / \mu \mathrm{L}(\%)$ & 3.81 & 0.96 & 4.35 & 0.63 & 3.97 & 0.55 \\
\hline Monocyte & $0.04-1.40$ & $10^{\wedge} 3 / \mu \mathrm{L}(\%)$ & 0.11 & 0.05 & 0.20 & 0.05 & 0.16 & 0.04 \\
\hline Eosinophil & $0.00-0.51$ & $10^{\wedge} 3 / \mu \mathrm{L}(\%)$ & 0.26 & 0.15 & 0.36 & 0.14 & 0.26 & 0.06 \\
\hline Basophil & $0.00-0.12$ & $10^{\wedge} 3 / \mu \mathrm{L}(\%)$ & 0.033 & 0.020 & 0.038 & 0.015 & 0.032 & 0.020 \\
\hline Neutrophil & $6.5-50.0$ & $\%$ & $14.44 \%$ & 0.06 & $15.73 \%$ & 0.02 & $16.02 \%$ & 0.04 \\
\hline Lymphocyte & $40.0-92.0$ & $\%$ & $77.61 \%$ & 0.06 & $74.15 \%$ & 0.04 & $75.25 \%$ & 0.03 \\
\hline Monocyte & 0.9-18.0 & $\%$ & $2.23 \%$ & 0.01 & $3.35 \%$ & 0.00 & $3.10 \%$ & 0.01 \\
\hline Eosinophil & $0.0-7.5$ & $\%$ & $4.96 \%$ & 0.02 & $6.13 \%$ & 0.02 & $5.03 \%$ & 0.01 \\
\hline Basophil & $0.0-1.5$ & $\%$ & $0.76 \%$ & 0.00 & $0.65 \%$ & 0.00 & $0.60 \%$ & 0.00 \\
\hline \multicolumn{9}{|l|}{ Biochemistry } \\
\hline ALT & 27-195 & $\mathrm{U} / \mathrm{L}$ & 49.000 & 28.678 & $148.286^{\mathrm{a}}$ & 99.876 & $103.200^{\mathrm{b}}$ & 157.301 \\
\hline Alb & $2.4-4.3$ & $\mathrm{~g} / \mathrm{dL}$ & 3.186 & 0.069 & 3.043 & 0.127 & 3.160 & 0.055 \\
\hline GGT & $0-9$ & $\mathrm{U} / \mathrm{L}$ & $<3$ & 0.000 & $<3$ & 0.000 & $<3$ & 0.000 \\
\hline
\end{tabular}

${ }^{\mathrm{a} A L T}$ value was $312 \mathrm{U} / \mathrm{L}$ in one mouse in $5 \times$ MIC CAMP-A treatment group.

${ }^{\mathrm{b}} \mathrm{ALT}$ value was $384 \mathrm{U} / \mathrm{L}$ in one mouse in 50×MIC CAMP-A treatment group.

WBC: white blood cells; RBC: red blood cells; HGB: hemoglobin; HCT: hematocrit; MCV: mean cell volume; $\mathrm{MCH}$ : mean cell hemoglobin; MCHC: mean cell hemoglobin; RDW: red cell distribution width; ALT: alanine transferase; ALB: albumin; GGT: gammaglutamyltransferase. 


\section{Histopathological findings}

The inflammation of wounded skin involved the epidermis, dermis, subcutis, and fibrous connective tissue, varied from moderate to severe. However, there was no significant difference found among the three treatment groups: 4×MIC CAMP-A, 4×MIC CAMP-B, and $0.9 \% \mathrm{NaCl}$ (Figure 5-3A to 3C). No tissue damage and significant inflammatory infiltration were observed in healthy (non-wounded) skin collected from control mice (Figure 5-3D). The thickness of epidermis and dermis of wounded skin was similar among three treatment groups but significantly thicker than that of the healthy skin. The average scores of the inflammatory response based on infiltrating neutrophils and monocytes were similar among three treatment groups (Figure 5-3E). No CAMP-specific lesion or inflammation was observed in the liver and kidneys (data was not shown). The data indicated that administration 4XMIC of CAMP-A and CAMP-B did not induce any obvious toxicity to skin, liver, and kidneys of the experimental mice. Similarly, treatment with higher doses of CAMP-A $(5 \times$ or $50 \times$ MIC) did not cause a significant increase in the inflammatory scores of wounded skin, compared to the treatment with $0.9 \% \mathrm{NaCl}$. No remarkable lesion was observed in the kidneys following treatment with high doses of CAMP-A. A few eosinophils and binucleate cells were observed in the liver of one mouse in the $50 \times \mathrm{MIC}$ of CAMP-A group, which were also observed in the livers of 2 mice in $\mathrm{NaCl}$ and polysporin-treated groups. Therefore, infiltration of these cells was less likely caused by CAMP-A toxicity (data not shown). 

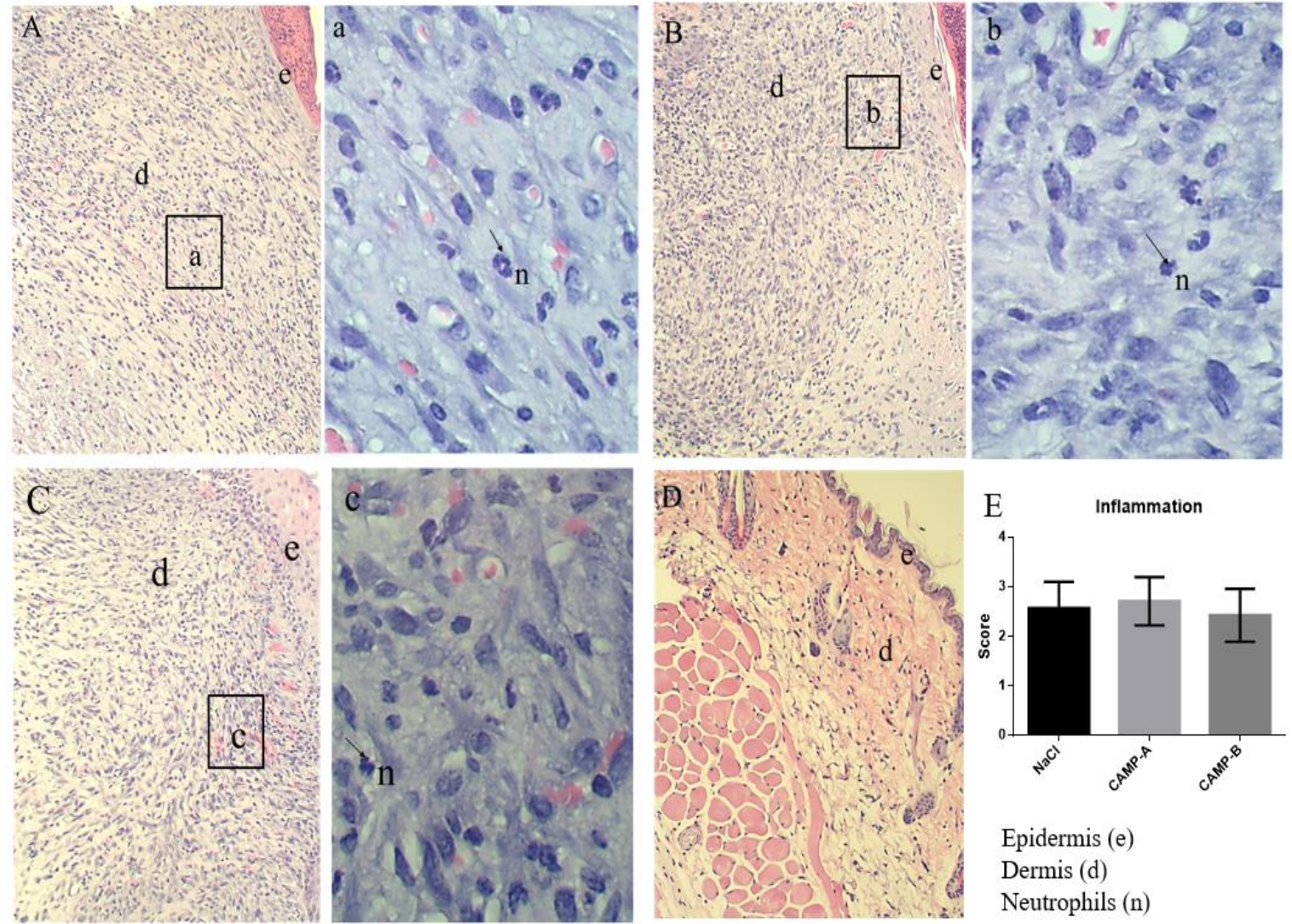

Epidermis (e)

Dermis (d)

Neutrophils (n)

\section{Figure 5-3. Histology of wounded skin following treatment with CAMPs}

Wounded skin tissues were excised on day 7 post initial treatment and immediately fixed in $10 \%$ of formalin, embedded in paraffin, sectioned at $5-\mu \mathrm{m}$ thickness, and stained with hematoxylin and eosin (H\&E). Treatment with $0.9 \% \mathrm{NaCl}(\mathrm{A}), 4 \times \mathrm{MIC}$ CAMP-A (B), 4×MIC CAMP-B (C), 10× magnification and 40× magnification in boxed area. (D) Healthy (non-wounded) skin. (E) Scores of the inflammatory responses in three groups. The inflammatory infiltration consisted of lymphocytes, macrophages, and neutrophils. Letters indicate the following cell types: e, epidermis; d, dermis; n, neutrophils. 


\section{Clearance of bacterial infection by CAMP-A and CAMP-B}

In the first trial, there was no significant difference in body weight and visual inspection scores among four groups $(2 \times$ MIC CAMP-A, $2 \times$ MIC CAMP-B, polysporin, and $0.9 \%$ $\mathrm{NaCl}$ ) at any time point (Figure 5-4). On day 1 post initial treatment, the bacterial counts in the wounds treated with CAMP-A and CAMP-B were $6.2 \log _{10}$ CFU/g and $6.6 \log _{10}$ CFU/g, respectively, whereas the bacterial counts in wounds treated with polysporin and $0.9 \% \mathrm{NaCl}$ were $4.2 \log _{10} \mathrm{CFU} / \mathrm{g}$ and $7.53 \log _{10} \mathrm{CFU} / \mathrm{g}$, respectively $(p<0.05$, Figure 55A). On day 3, the bacterial counts in wounds treated with CAMP-A, CAMP-B, and polysporin reduced to $5.18,6.6$, and $2.7 \log _{10} \mathrm{CFU} / \mathrm{g}$, respectively, while the bacterial count in wounds treated with $0.9 \% \mathrm{NaCl}$ increased to $8.1 \mathrm{CFU} / \mathrm{g}(p<0.05$, Figure 5-5B). On day 5, the bacterial counts in CAMP-A, CAMP-B, and polysporin groups further reduced to 4.3, 5.8, and $1.7 \log _{10} \mathrm{CFU} / \mathrm{g}$, respectively, and the bacterial count in $0.9 \% \mathrm{NaCl}$ group peaked at $8.1 \mathrm{CFU} / \mathrm{g}(p<0.01$, Figure 5-5C). Treatment with CAMP-A and polysporin completely prevented bacterial infection in the liver and kidneys (Figure 5-6). Following treatment with CAMP-B, systemic dissemination did not occur till day 5 while the mocktreatment $(0.9 \% \mathrm{NaCl})$ group developed systemic infection within 48 hours. By day 5, CAMP-B blocked systemic infection in 4 of 6 (67\%) mice (Figure 5-6). In control group $(0.9 \% \mathrm{NaCl})$, liver infection showed in all 6 mice by day 3 (Figure 5-6A) and kidney infection occurred in all 6 mice by day 5 (Figure 5-6B). In summary, CAMP-A reduced wound bacterial burden and prevented bacterial dissemination from wounds to systemic organs and CAMP-B reduced wound bacterial burden and prevented systemic infection in $67 \%$ of mice. The efficacy of these compounds could be ranked as polysporin > CAMP$\mathrm{A}>\mathrm{CAMP}-\mathrm{B}>\mathrm{NaCl}$. 


\section{Histology of skin post-infection and treatment}

Inoculation of $P$. aeruginosa on the full-thickness wound (removed epidermis and dermis layer of skin), server inflammatory response was evident among four groups, independent of the treatment $(2 \times \mathrm{MIC}$ CAMP-A, $2 \times \mathrm{MIC}$ CAMP-B, or $0.9 \% \mathrm{NaCl})$. Post-3-d treatment, epidermal cells, and connective tissues cells started to proliferate to fill the wound, the

diameter of the open wound was significantly different among groups (Figure 5-5B): Polysporin $<2 \times$ MIC CAMP-A and CAMP-B $<\mathrm{NaCl}$. The inflammatory response was most intense in the subcutaneous tissues infiltrated neutrophils. On day 5 , the epidermal cells proliferated to healing the wound at some CAMPs- and polysporin-treated mice, but was less evident in NaCl-treated mice. The inflammatory response showed a similar trend with the closure of wounds post-treatment, showing less severity in the treated groups with CAMPs and polysporin (Figure 5-5C). 

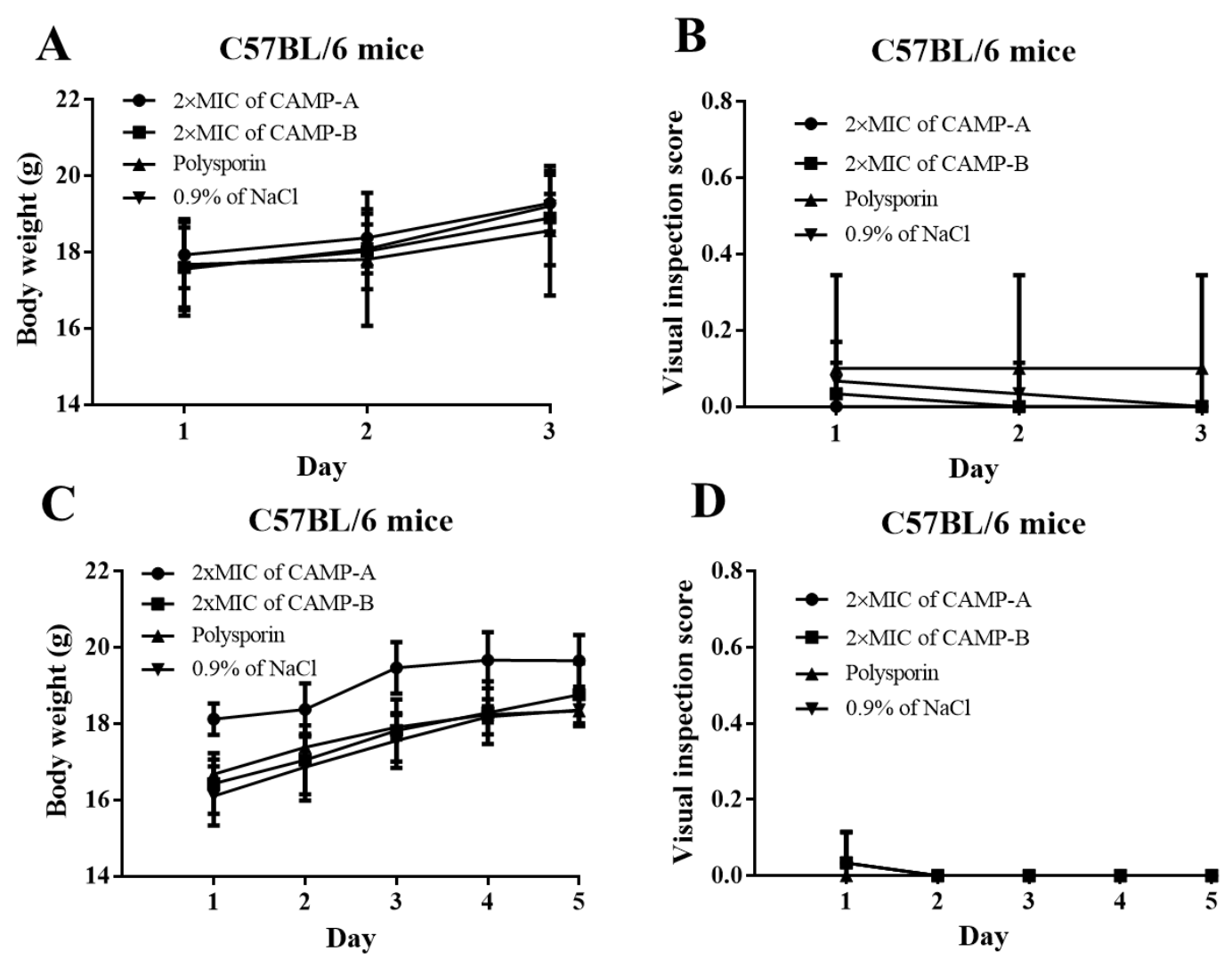

Figure 5-4. Body weight and visual inspection scores of treated mice

Each experiment included four treatment groups. Four hours post-inoculation of the wound with $2.5 \times 10^{6} \mathrm{CFU}$ of $P$. aeruginosa, wounds of mice in different groups were treated daily with CAMP-A $(2 \times \mathrm{MIC}), \mathrm{CAMP}-\mathrm{B}(2 \times \mathrm{MIC})$, polysporin, and $0.9 \% \mathrm{NaCl}$. (A) Body weight of mice and (B) visual inspection scores of physical activities, and smoothness of coat of mice on day 3 post-infection. (C) Body weight of mice and (D) variation of visual inspection of the activity and smoothness of coated hair of mice on day 5 post-infection. 

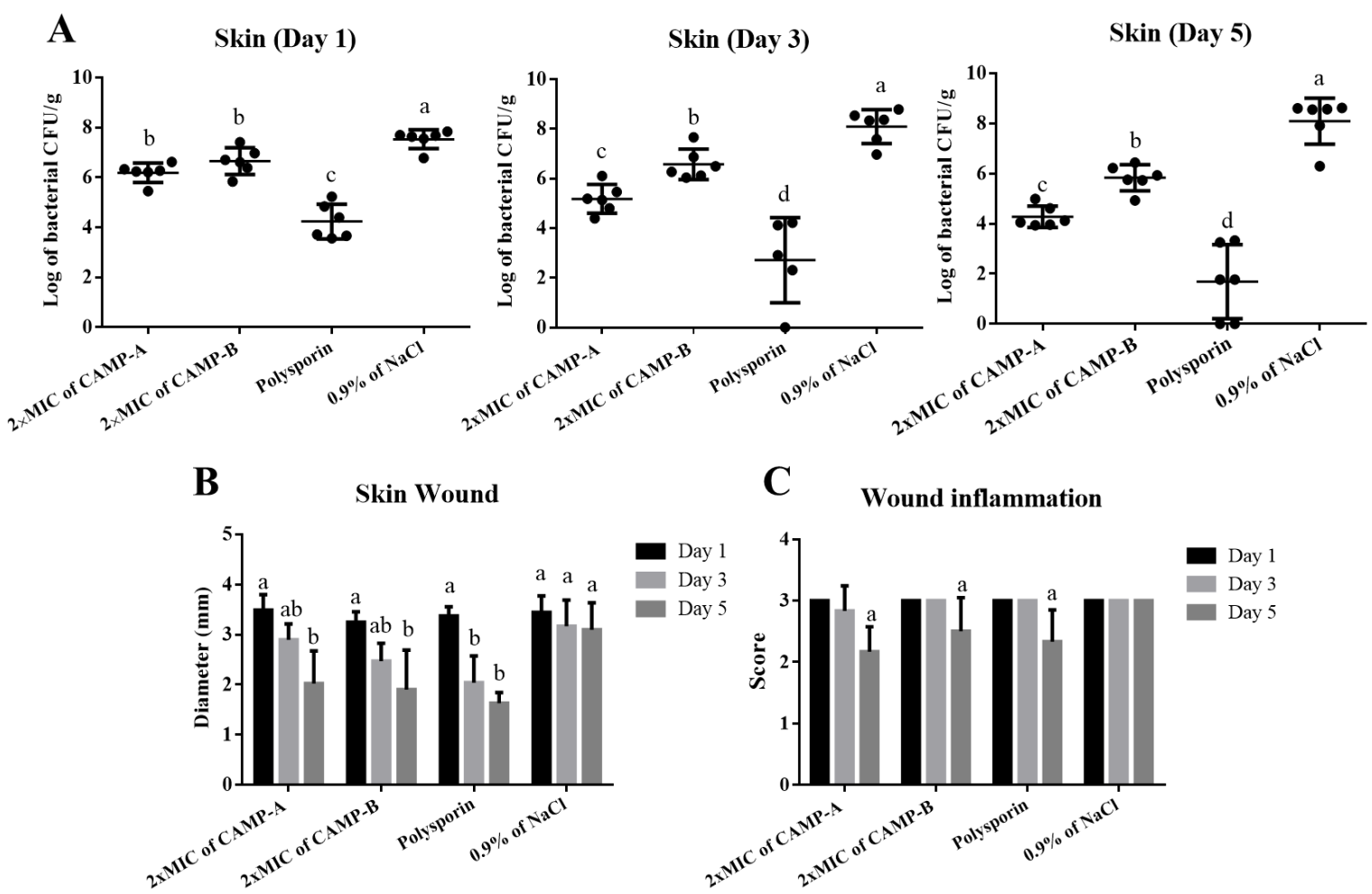

Figure 5-5. The bacterial load in skin wound following treatment with CAMP-A and

\section{CAMP-B $(2 \times$ MIC $)$}

Skin wounds were infected with $2.5 \times 10^{6} \mathrm{CFU}$ of $P$. aeruginosa and treated daily with CAMP-A ( $2 \times$ MIC), CAMP-B ( $2 \times \mathrm{MIC})$, polysporin, or $0.9 \% \mathrm{NaCl}$. (A) Bacterial counts $\left(\log _{10}\right.$ of bacterial CFU/g skin) in skin tissue homogenates on day 1, day 3 , and day 5. (B) Diameter of the wounds as an indicator of wound closure. (C) Inflammatory scores of the infected wound skin. Data are presented as means $\pm S D(n=6)$. Lowercase letters $(a, b, c$, and d) represent significant difference among treatment groups $(p<0.05)$. 

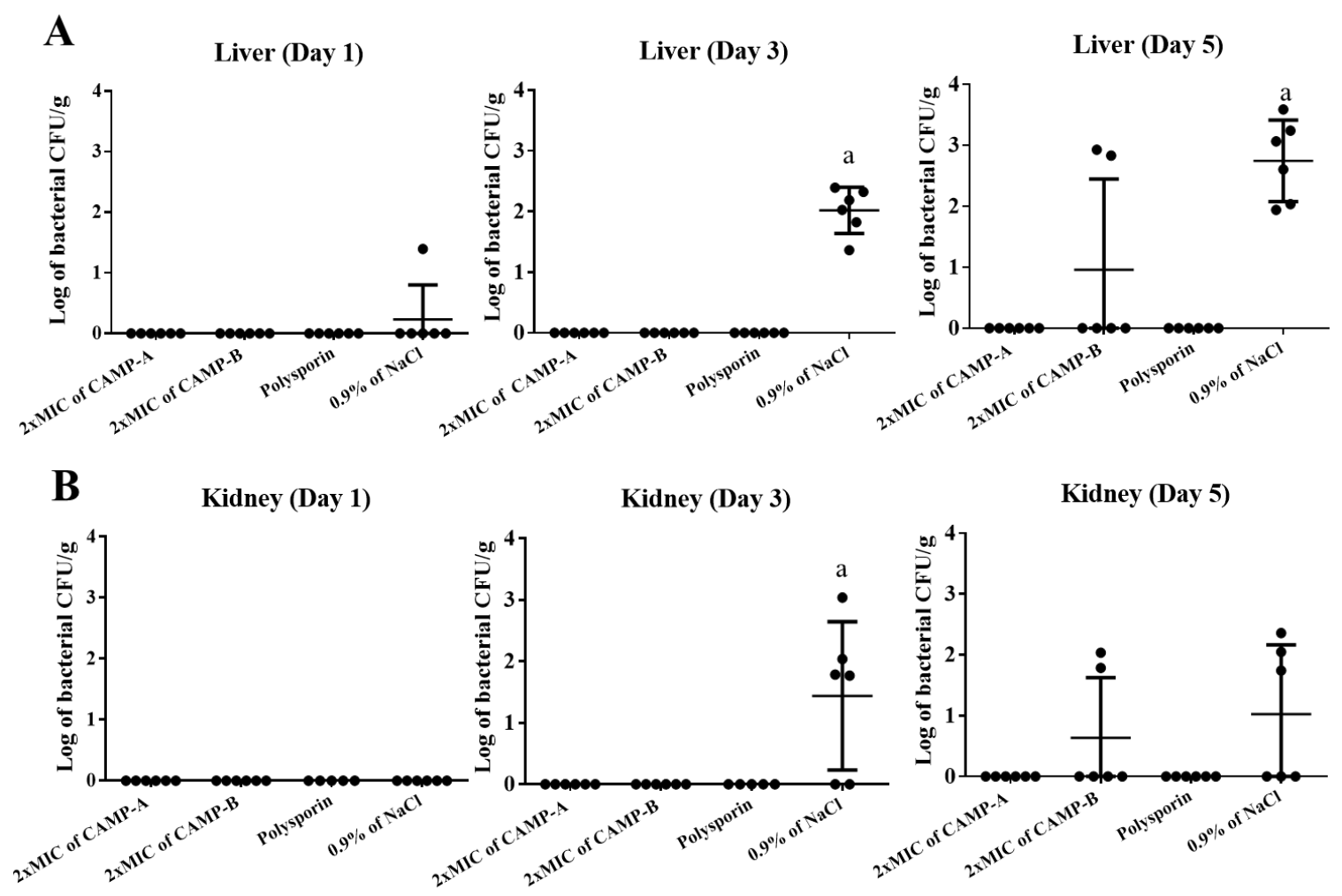

Figure 5-6. Efficacy of CAMP-A and CAMP-B $(2 \times M I C)$ in prevention of $P$. aeruginosa infection in the liver and kidneys

Skin wounds of mice were infected with $2.5 \times 10^{6} \mathrm{CFU}$ of $P$. aeruginosa and treated daily with CAMP-A (2×MIC), CAMP-B ( $2 \times \mathrm{MIC})$, polysporin, and $0.9 \% \mathrm{NaCl}$, respectively. Viable bacterial counts ( $\log _{10}$ of bacterial CFU/g tissue) in the liver (A) and kidney (B) tissue homogenates on day 1 , day 3 , and day 5 post-infection. Data are presented as means $\pm \operatorname{SD}(n=6)$. Lowercase letter (a) indicate significant difference among treatments $(p<$ $0.05)$. 


\section{Efficacy of CAMP-A at higher concentrations}

When high doses of CAMP-A (5x and 50x MIC) were used to treat skin wounds, no significant differences were observed among treatment groups in body weight (Figure 57A), visual inspection scores (Figure 5-7B), and inflammatory scores (Figure 5-7D). Treatment with 50×MIC CAMP-A slightly improved wound healing, compared to 5×MIC CAMP-A and $0.9 \% \mathrm{NaCl}$ (Figure 5-7C). On day 5, the bacterial counts in wounds treated with 5×MIC CAMP-A, 50×MIC CAMP-A, polysporin, and 0.9\% $\mathrm{NaCl}$ were 4.5, 3.8, 2.2 and $8.6 \log _{10} \mathrm{CFU} / \mathrm{g}$, respectively. CAMP-A at both concentrations (5×MIC and 50×MIC) and polysporin inhibited $P$. aeruginosa infection in the liver and kidneys of mice (Figure

5-8). In the control group (treatment with $0.9 \% \mathrm{NaCl}$ ), 5 of 6 mice were infected in the livers and 3 of 6 mice were infected in the kidneys on day 5 (Figure 5-8). 


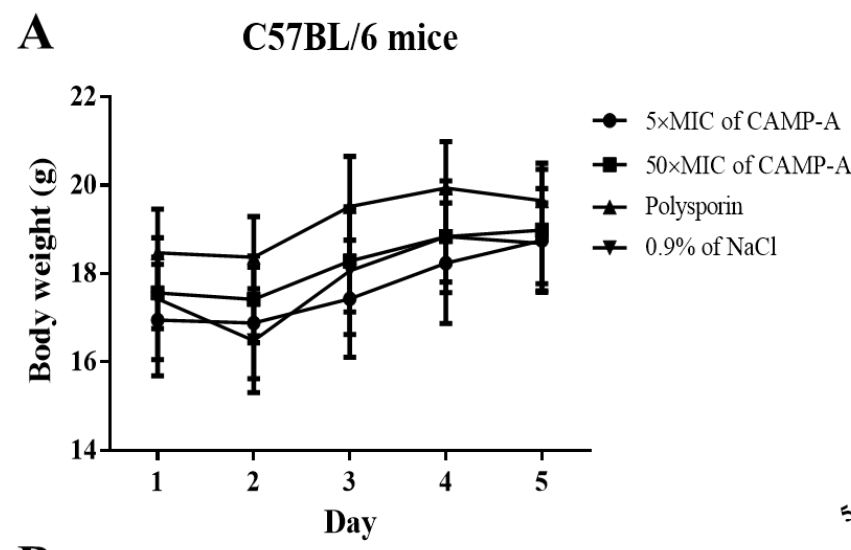

C Skin Wound (Day 5)
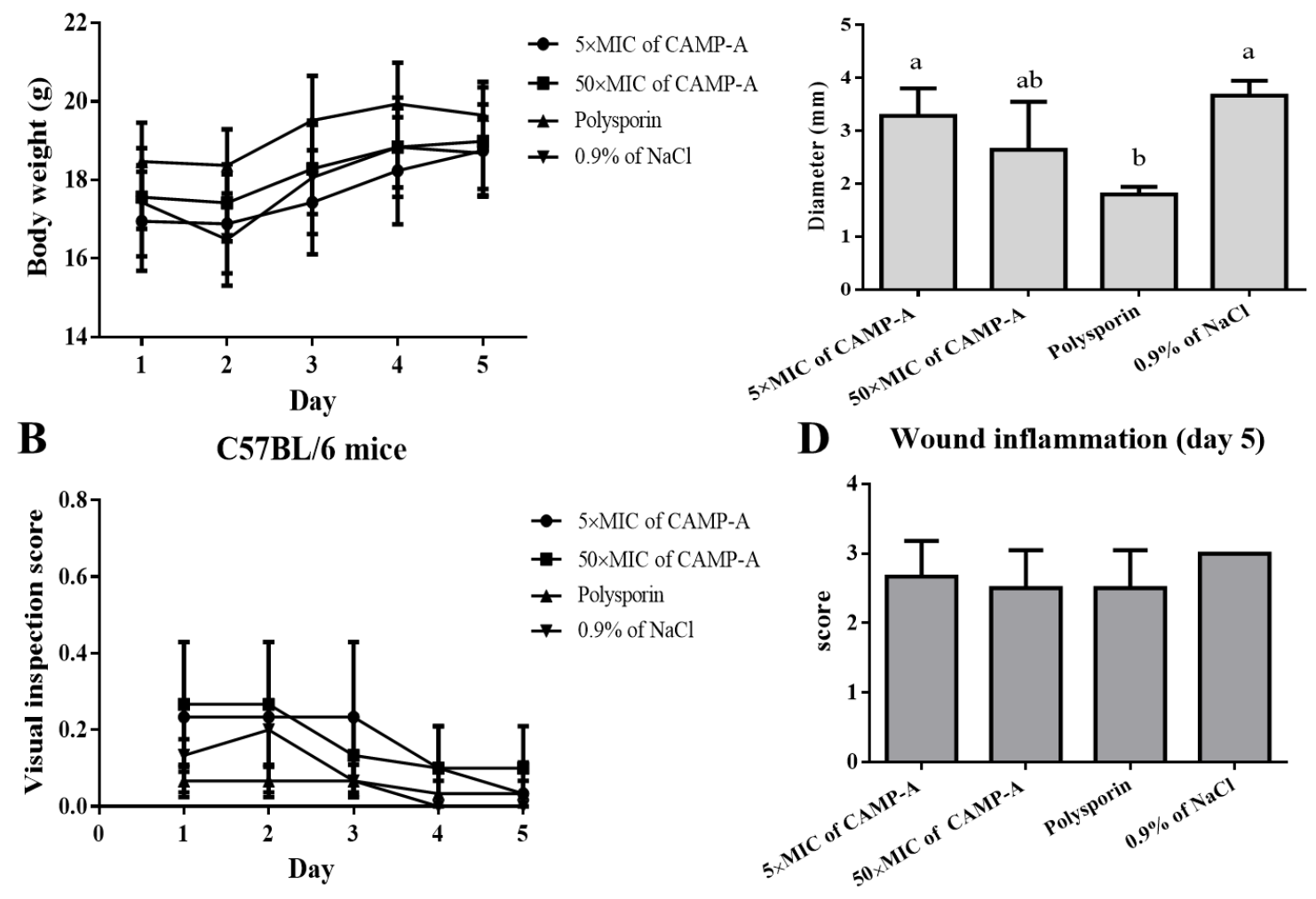

D Wound inflammation (day 5)

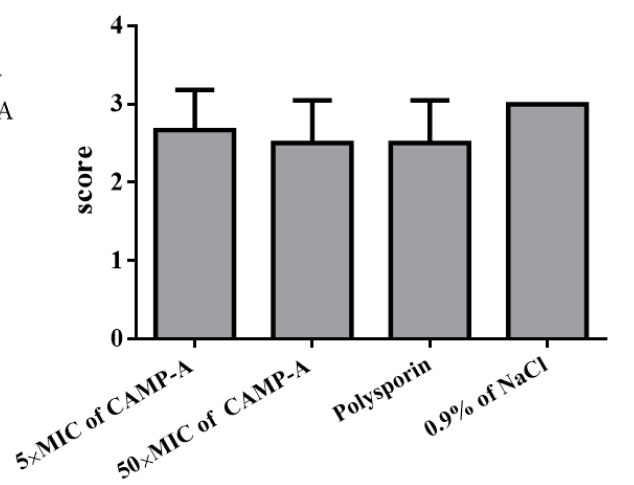

Figure 5-7. Body weight, visual inspection scores, wound closure, and inflammatory scores of mice treated with higher doses of CAMP-A

The four groups of mice were infected with $2.5 \times 10^{6} \mathrm{CFU}$ of $P$. aeruginosa and treated daily with CAMP-A (5×MIC), CAMP-A $(50 \times \mathrm{MIC})$, polysporin, and $0.9 \% \mathrm{NaCl}$, respectively. (A) Body weight of mice. (B) Visual inspection of activity and smoothness of skin hair. (C) Diameter of wounds as an indicator of wound healing. (D) The inflammatory scores of the infected skin. Data are presented as means $\pm \operatorname{SD}(n=6)$. Lowercase letters ( $a$ and $b)$ indicate significant difference among treatment groups $(p<$ $0.05)$. 


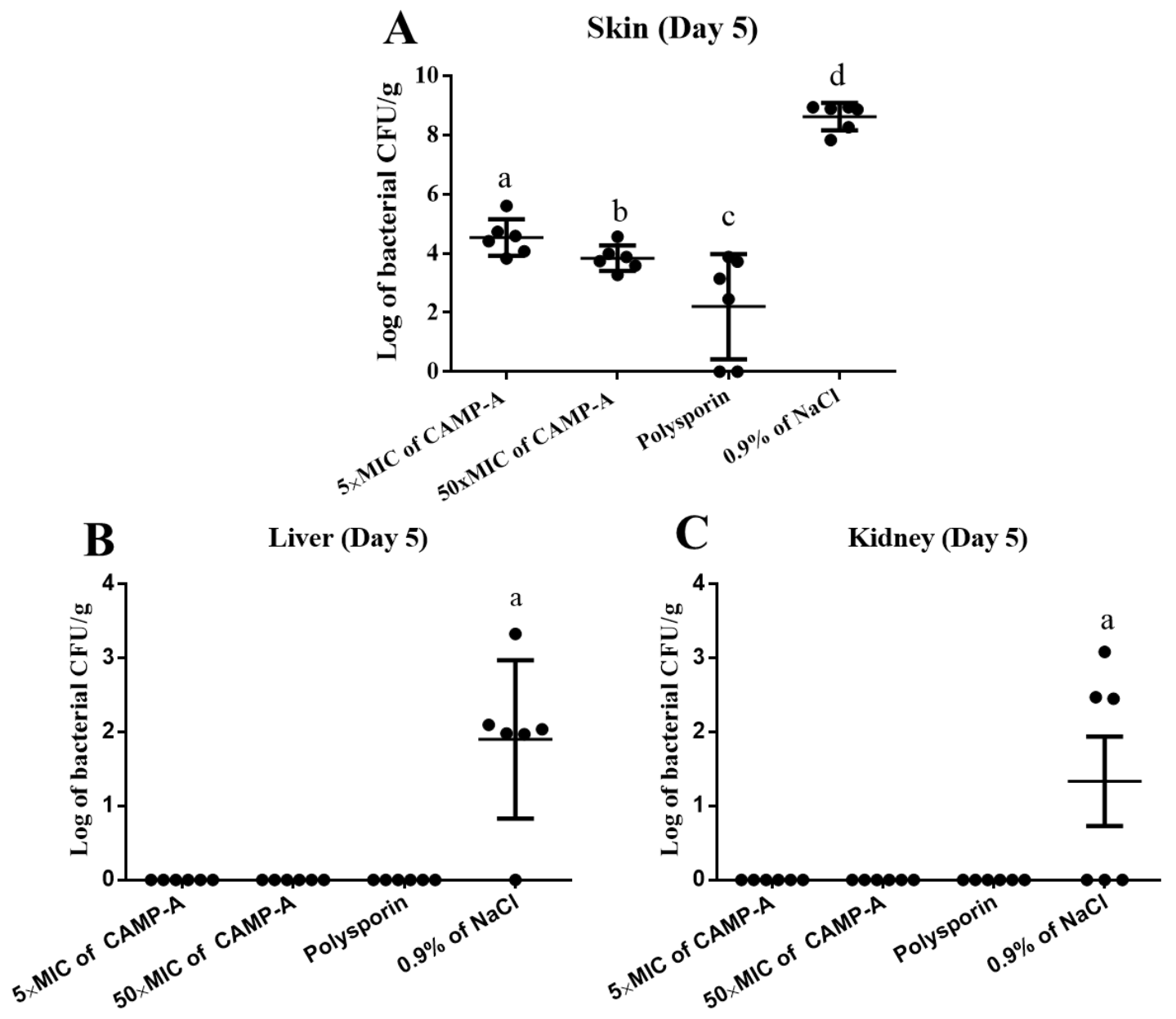

Figure 5-8. The efficacy of CAMP-A $(5 \times M I C$ and $50 \times M I C)$ in the treatment of $P$. aeruginosa infection

The skin wounds were infected with $2.5 \times 10^{6} \mathrm{CFU}$ of $P$. aeruginosa and treated daily with CAMP-A (5×MIC), CAMP-A (50×MIC), polysporin, and $0.9 \% \mathrm{NaCl}$, respectively. (A) Bacterial counts ( $\log _{10}$ of bacterial CFU/g skin) in the skin wound, (B) liver, and (C) kidneys. Data are presented as means \pm SD $(n=6)$. Lowercase letters $(a, b, c$, and $d)$ indicate significant difference among treatments $(p<0.05)$. 


\section{Discussion}

In the present study, an integrated strategy was applied to design novel antimicrobial peptides possessing desirable characteristics as therapeutic agents. The data from in vitro studies indicated that CAMP-A and CAMP-B were broad-spectrum, strong antimicrobial peptides with resistance to cationic salts and proteases. These two peptides were then subjected to in vivo characterizations using a mouse skin wound infection model. It is known that skin, as a physical barrier, plays an important role in protecting the host from microbial invasion. When this barrier is compromised, such as burn or surgical injuries, opportunistic pathogens can infect the wound and then spread to systemic sites, if wound is not properly treated [203, 204]. Among the known opportunistic pathogens, $P$. aeruginosa is a leading cause of secondary infection in immunocompromised hosts [190]. To ensure the establishment of wound and systemic infections, a relatively high dose of $P$. aeruginosa $\left(2.5 \times 10^{6} \mathrm{CFU}\right)$ was inoculated into each wound and waited 4 hours prior to topical application of peptides. Bacterial cultural results indicated that $P$. aeruginosa proliferated rapidly in the surgical wound and spread to the liver and kidneys by 48 hours in all experimental mice.

The toxicity of CAMP-A and CAMP-B was evaluated because this unfadable property could be a major hindrance in the application of antimicrobial peptides in therapy [205]. Hematology, complete blood count (CBC), is critical for the early identification of illness or disease. Variations in the size, shape, and number of blood cells indicate the effect of clinical factors or medicines [206]. Analysis of blood enzymes is usually to conducted to evaluate the functional variation of the liver and kidneys [207]. Therefore, hematological and blood biochemical profiles were analyzed to exam potential toxic of peptides. The 
results indicated that daily treatment with $4 \times$ MIC CAMP-A and CAMP-B for up to 7 days had no significant impact on the physical activities, body weight, hematological and blood biochemical profiles, and histopathology of wounded skin and systemic organs of mice. At higher doses (5× and 50× MIC), elevation of alanine aminotransferase (ALT), an indicator of liver damage, was detected in 1 of 7 mice in each treatment group. However, partial hemolysis was observed in these samples. It is unclear the elevated ALT was caused by CAMP treatment or hemolysis [208].

The efficacy of CAMP-A and CAMP-B was evaluated by quantifying bacterial load, wound closure, inflammatory infiltration in wounded skin, liver and kidney tissues. Polysporin ointment containing polymyxin B (a lipopeptide antibiotic) and bacitracin was included as a positive control because it is commonly used to treat and prevent wound infection, although the nephrotoxicity and neurotoxicity of polymyxin $\mathrm{B}$ has been a concern [209, 210, 211]. Wound healing processes are generally composed of inflammatory, proliferation and remodeling phases [212]. Accordingly, in the first trial, peptides were applied for different time points: 1 day, 3 days, and 5 days. Daily treatment with $2 \times$ MIC CAMP-A and CAMP-B reduced wound bacterial load in a time-dependent manner. CAMP-A completely prevented systemic dissemination of $P$. aeruginosa and CAMP-B delayed systemic dissemination by 2 days and prevented systemic infection in $67 \%$ of mice. Daily treatment with $2 \times$ MIC CAMP-A and CAMP-B for 5 days also facilitated wound closure and reduced inflammatory infiltration, and similar results were seen in polymyxin treatment group. Although inflammatory response was seen throughout the course of experiment, which was expected due to surgical trauma and P. aeruginosa infection [213]. To determine whether treatment efficacy was dose-dependent, a second 
trial was carried out. Treatment with 50×MIC CAMP-A for consecutive days further reduced wound bacterial load, compared to treatment with $2 \times$ MIC or $5 \times$ MIC CAMP-A. However, complete eradication of $P$. aeruginosa from wounds was not achieved by either CAMP-A or polysporin, suggesting that longer treatment might be necessary as reported previously [214]. In addition, treatment with higher dose of CAMP-A (5×MIC and $50 \times$ MIC) slightly delayed wound healing than treatment with $2 \times$ MIC CAMP-A, which might be caused due to the cytotoxicity of CAMP-A at higher dose to topical epithelial cells or other immune functions of CAMP-A.

In this study, CAMP-A and CAMP-B were less effective $(p<0.05)$ than a commercially available polysporin ointment in reducing bacterial burden in the infected wounds. Two factors might have contributed to this outcome. First, polysporin ointment contains 10, 000 units $(1 \mathrm{mg})$ of polymyxin B and 500 units $(6.75 \mathrm{mg})$ of bacitracin/g which are 9.6 to 242 times higher than the concentrations of CAMP-A and CAMP-B used in this study. Second, the ointment ensured the sustained interaction of drugs with bacteria in wounded tissue. Overall, the data suggest that CAMP-A is potentially an alternative to commonly used antibiotics to treat $P$. aeruginosa infection upon further development.

\section{Conclusions}

In conclusion, CAMP-A and CAMP-B did not cause acute toxicity in experimental mice, except elevated ALT level in 14\% of mice. Both CAMP-A and CAMP-B reduced wound bacterial load. Topical treatment with CAMP-A completely prevented bacterial dissemination from the infected wound to systemic organs whereas CAMP-B prevented systemic infection in $67 \%$ of mice. 


\section{CHAPTER 6: Summary}

Antimicrobial peptides (AMPs) constitute a key component of the innate immune system, which have been isolated from many organisms including plants, insects, and animals. AMPs show a broad-spectrum antimicrobial activity against bacteria, fungi, viruses, protozoa, and even cancer cells, also called host defense peptides. They exert their antimicrobial activity by selectively interacting with the negatively charged microbial membrane, increasing membrane permeability, penetrating membrane to inhibit DNA, RNA, and protein synthesis. Some AMPs also exhibit immunomodulatory functions by neutralizing bacterial cell wall components (e.g. LPS), stimulating the secretion of proinflammatory cytokines, and chemoattracting immune cells such as lymphocytes, macrophages, and immature dendritic cells. With pleiotropic antimicrobial mechanisms, it is more difficult for bacteria to develop antimicrobial resistance to AMPs. Therefore, AMPs have been considered as alternatives to conventional antibiotics.

In past decades, hundreds of natural AMPs have been shown to have antimicrobial activity against microbial pathogens. Due to certain impeding factors such as cytotoxicity to host cells, high manufacturing cost, and ineffectiveness of peptides in physiological conditions, only a few AMPs or AMP-like peptides have been applied in the clinical settings, these drawbacks must be overcome in order to develop AMPs as novel therapeutic agents against infections, especially those caused by antibiotic-resistant microbes.

In this dissertation, we initially investigated the structure-function relationship of two less studied avian $\beta$-defensins (AvBDs), AvBD-6 and AvBD-12. They were chosen based on

the tissue expressing patterns and net positive charges: AvBD-6 (+7) is mainly expressed in bone marrow while AvBD-12 (+1) is strongly expressed in epithelial cells. The 
antimicrobial results showed that AvBD-6 was more potent than AvBD-12 against E. coli, S. Typhimurium, and S. aureus as well as clinical isolates of extended-spectrum betalactamase (ESBL)-producing E. coli and K. pneumoniae, whereas AvBD-12 showed higher chemotactic ability to chicken macrophages and mouse immature dendritic cells than that of AvBD-6. Neither AvBDs in effective killing doses had cytotoxic effect on host cells. However, like other defensins, their antimicrobial activity was diminished in physiological centration of cationic salts and chemotactic activities were compromised without disulfide bonds.

We further dissected the roles of charge, hydrophobicity, and disulfide bonds in the antimicrobial and chemotactic activities of AvBDs by designing and analyzing a series of AvBD analogues that had net positive charges of +1 to +9 , hydrophobicity of $33 \%$ to $53 \%$, and disulfide bonds of 0 to 3 . Functional characterization of the analogues revealed that the net positive charge and charge distribution had a dominating effect on the antimicrobial potency of AvBDs, the three conserved disulfide bonds were essential to chemotactic property as well as maximum antimicrobial activity. The chemotactic activity was associated with the $\mathrm{N}$-terminal $\alpha$-helix and an adjacent $\beta 2-\beta 3$ loop which had been reported as the binding domain for human chemokine receptor CCR2. AvBD-12A3, a linear analogue, demonstrated strongest antimicrobial activity, improved salt resistance, and mild chemotactic property. This analogue with a high net positive charge $(+9)$, a moderate degree of hydrophobicity (40\%) and a CCR2-binding domain could serve as a template to design novel antimicrobial peptides.

In the following study, we designed a series of short cationic antimicrobial peptides (CAMPs) by extracting the key functional components of AvBDs and incorporating 
additional favorable characteristics. CAMP-t1 consisted the CCR2 binding domain (Nterminal $\alpha$-helix and adjacent loop) of AvBD-12 with increased number of positively charged residues for both chemotactic and antimicrobial properties. CAMP-t2 was comprised of the key a.a. residues contributing to the concentrated positive surface charge and hydrophobicity of AvBD-6 for antimicrobial property. Functional analysis of these peptides revealed that CAMP-t1 and CAMP-t 2 were more potent than the wild-type AvBDs against $P$. aeruginosa and Staphylococcus spp. However, CAMP-t1 with a predicted $\mathrm{N}$-terminal helix and adjacent loop failed to show any chemotactic activity. The research was then focused on improving the antimicrobial activity of CAMPs. As such, CAMP-t2 was further modified to yield two $\alpha$-helical peptides CAMP-A and CAMP-B which lacked potential protease cutting sites and possessed a C-terminal poly-Trp tail, Nterminal acetylation, and C-terminal amidation. In vitro characterizations showed that CAMP-A and CAMP-B had significantly improved antimicrobial activity against $P$. aeruginosa and Staphylococcus aureus as well as multidrug-resistant $P$. aeruginosa and methicillin-resistant S. pseudintermedius strains (MRSP). CAMP-A and CAMP-B demonstrated following advantageous characteristics: rapid membrane attacking (30 min), resistance to physiological concentrations of $\mathrm{NaCl}$ and $\mathrm{CaCl}_{2}$, resistance or tolerance to protease degradation, and a therapeutic index of 4.5 or greater, suggesting potentially safe for clinical use.

Finally, we evaluated the acute toxicity and antimicrobial efficacy of CAMP-A and CAMP-B using a mouse wound infection (P. aeruginosa) model. Daily application of CAMP-A and CAMP-B on surgical wounds at doses of $4 \times, 5 \times$ and $50 \times$ MIC did not induce any signs of acute toxicity. Following CAMP treatment, no significant changes was 
detected in physical activity, body weight, histology of local and systemic tissues, hematological parameters, and blood biochemical profiles, except that CAMP-A at higher doses $(5 \times$ and $50 \times$ MIC) was associated with elevated ATL in $14 \%$ of mice. Daily treatment of infected wounds with $2 \times$ MIC CAMP-A and CAMP-B reduced the bacterial burden in wounds in a time-dependent manner. Treatment with CAMP-A completely prevented systemic infection by $P$. aeruginosa while treatment with CAMP-B delayed systemic dissemination by 2 days and prevented systemic infection in $67 \%$ of mice. Treatment of infected wounds with CAMP-A and CAMP-B for 5 consecutive days also facilitated wound healing and reduced inflammatory infiltration in wounded skin tissue. At higher does (5x and 50× MIC), CAMP-A further reduced wound bacterial load, suggesting that the efficacy was dose-dependent. CAMP-A and CAMP-B were less effective than commercially available polysporin ointment which, however, contained polymyxin B and bacitracin at much higher concentrations. In conclusion, CAMPs can be developed as effective therapeutic agents by incorporating advantageous characteristics and eliminating undesired features. When design and modify CAMPs, a comprehensive and integrated approach should be used to maximize desired biological functions. The peptides designed in the present study, especially CAMP-A, may serve as alternatives to traditional antimicrobial agents, or used in combination with other antibiotics, in the treatment and prevention of microbial infections. 


\section{REFERENCES}

1. Bren L: Battle of the bugs: fighting antibiotic resistance. FDA Consumer magazine 2002, 36(4):28-34.

2. Brunetti J, Falciani C, Bracci L, Pini A: Models of in-vivo Bacterial infections for the development of antimicrobial peptide-based drugs. Current topics in medicinal chemistry 2017, 17(5):613-619.

3. Ventola CL: The antibiotic resistance crisis: part 1: causes and threats. Pharmacy and Therapeutics 2015, 40(4):277-283.

4. Zasloff M: Antimicrobial peptides of multicellular organisms. Nature 2002, 415(6870):389-395.

5. Wang Z, Wang G: APD: the antimicrobial peptide database. Nucleic Acids Research 2004, 32(Database issue): D590-592.

6. Wang G, Li X, Wang Z: APD3: the antimicrobial peptide database as a tool for research and education. Nucleic Acids Research 2016, 44(Database issue): D1087D1093.

7. Hirsch JG: Phagocytin: a bactericidal substance from polymorphonuclear leucocytes. Journal of Experimental Medicine 1956, 103(5):589-611.

8. Brogden KA: Antimicrobial peptides: pore formers or metabolic inhibitors in bacteria? Nature Reviews Microbiology 2005, 3(3):238-250.

9. Zhang G, Sunkara LT: Avian antimicrobial host defense peptides: from biology to therapeutic applications. Pharmaceuticals (Basel) 2014, 7(3):220-247.

10. Huttner KM, Bevins CL: Antimicrobial peptides as mediators of epithelial host defense. Pediatric Research 1999, 45:785-794. 
11. Ganz T: Defensins: antimicrobial peptides of innate immunity. Nature Reviews Immunology 2003, 3(9):710-720.

12. Zhang LJ, Gallo RL: Antimicrobial Peptides. Current Biology 2016, 26(1):R14-19.

13. Lai R, Liu H, Hui Lee W, Zhang Y: An anionic antimicrobial peptide from toad Bombina maxima. Biochemical and Biophysical Research Communications 2002, 295(4):796-799.

14. Schittek B, Hipfel R, Sauer B, Bauer J, Kalbacher H, Stevanovic S, Schirle M, Schroeder K, Blin N, Meier F et al: Dermcidin: a novel human antibiotic peptide secreted by sweat glands. Nature Immunology 2001, 2(12):1133-1137.

15. Brogden KA, De Lucca AJ, Bland J, Elliott S: Isolation of an ovine pulmonary surfactant-associated anionic peptide bactericidal for Pasteurella haemolytica. Proceedings of the National Academy of Sciences of the United States of America 1996, 93(1):412-416.

16. Powers JP, Hancock RE: The relationship between peptide structure and antibacterial activity. Peptides 2003, 24(11):1681-1691.

17. Bahar A, Ren D: Antimicrobial peptides. Pharmaceuticals 2013, 6(12):1543-1575.

18. Rozek A, Friedrich CL, Hancock RE: Structure of the bovine antimicrobial peptide indolicidin bound to dodecylphosphocholine and sodium dodecyl sulfate micelles. Biochemistry 2000, 39(51):15765-15774.

19. Gesell J, Zasloff M, Opella SJ: Two-dimensional 1H NMR experiments show that the 23-residue magainin antibiotic peptide is an alpha-helix in dodecylphosphocholine micelles, sodium dodecylsulfate micelles, and trifluoroethanol/water solution. Journal of Biomolecular NMR 1997, 9(2):127-135. 
20. Hwang PM, Zhou N, Shan X, Arrowsmith CH, Vogel HJ: Three-dimensional solution structure of lactoferricin B, an antimicrobial peptide derived from bovine lactoferrin. Biochemistry 1998, 37(12):4288-4298.

21. Derache C, Meudal H, Aucagne V, Mark KJ, Cadene M, Delmas AF, Lalmanach AC, Landon C: Initial insights into structure-activity relationships of avian defensins. Journal of Biological Chemistry 2012, 287(10):7746-7755.

22. Teng D, Wang X, Xi D, Mao R, Zhang Y, Guan Q, Zhang J, Wang J: A dual mechanism involved in membrane and nucleic acid disruption of AvBD103b, a new avian defensin from the king penguin, against Salmonella enteritidis CVCC3377. Applied Microbiology and Biotechnology 2014, 98(19):8313-8325.

23. Lee J, Lee DG: Antimicrobial peptides (AMPs) with dual mechanisms: membrane disruption and apoptosis. Journal of Microbiology and Biotechnology 2015, 25(6):759-764.

24. Malanovic N, Lohner K: Antimicrobial peptides targeting gram-positive bacteria. Pharmaceuticals 2016, 9(3):59.

25. Yeaman MR, Yount NY: Mechanisms of antimicrobial peptide action and resistance. Pharmacological Reviews 2003, 55(1):27-55.

26. Madani F, Lindberg S, Langel U, Futaki S, Graslund A: Mechanisms of cellular uptake of cell-penetrating peptides. Journal of Biophysics 2011, 2011:414729.

27. Mihajlovic M, Lazaridis T: Antimicrobial peptides in toroidal and cylindrical pores. Biochimica et Biophysica Acta 2010, 1798(8):1485-1493.

28. Brandenburg L-O, Merres J, Albrecht L-J, Varoga D, Pufe T: Antimicrobial peptides: multifunctional drugs for different applications. Polymers 2012, 4(1):539. 
29. Huang Y, Huang J, Chen Y: Alpha-helical cationic antimicrobial peptides: relationships of structure and function. Protein \& cell 2010, 1(2):143-152.

30. Zasloff M: Antimicrobial peptides of multicellular organisms. Nature 2002, 415(6870):389-395.

31. Guilhelmelli F, Vilela N, Albuquerque P, Derengowski LdS, Silva-Pereira I, Kyaw CM: Antibiotic development challenges: the various mechanisms of action of antimicrobial peptides and of bacterial resistance. Frontiers in Microbiology 2013, 4:353.

32. Hancock RE, Sahl HG: Antimicrobial and host-defense peptides as new antiinfective therapeutic strategies. Nature Biotechnology 2006, 24(12):1551-1557.

33. Zhang YM, Rock CO: Transcriptional regulation in bacterial membrane lipid synthesis. Journal of lipid research 2009, 50 Supplement: S115-119.

34. Cho JH, Sung BH, Kim SC: Buforins: histone H2A-derived antimicrobial peptides from toad stomach. Biochimica et Biophysica Acta 2009, 1788(8):1564-1569.

35. Ageitos JM, Sánchez-Pérez A, Calo-Mata P, Villa TG: Antimicrobial peptides (AMPs): ancient compounds that represent novel weapons in the fight against bacteria. Biochemical Pharmacology 2017, 133:117-138.

36. Boman HG, Agerberth B, Boman A: Mechanisms of action on Escherichia coli of cecropin P1 and PR-39, two antibacterial peptides from pig intestine. Infection and Immunity 1993, 61(7):2978-2984.

37. Yang D, Biragyn A, Kwak LW, Oppenheim JJ: Mammalian defensins in immunity: more than just microbicidal. Trends in Immunology 2002, 23(6):291-296. 
38. Selsted ME, Ouellette AJ: Mammalian defensins in the antimicrobial immune response. Nature Immunology 2005, 6(6):551-557.

39. Lehrer RI: Primate defensins. Nature Reviews Microbiology 2004, 2(9):727-738.

40. Xiao Y, Hughes AL, Ando J, Matsuda Y, Cheng JF, Skinner-Noble D, Zhang G: A genome-wide screen identifies a single beta-defensin gene cluster in the chicken: implications for the origin and evolution of mammalian defensins. BMC Genomics 2004, 5(1):56.

41. Bauer F, Schweimer K, Klüver E, Conejo-Garcia J-R, Forssmann W-G, Rösch P, Adermann K, Sticht H: Structure determination of human and murine $\beta$-defensins reveals structural conservation in the absence of significant sequence similarity. Protein Science: A Publication of the Protein Society 2001, 10(12):2470-2479.

42. Landon C, Thouzeau C, Labbe H, Bulet P, Vovelle F: Solution structure of spheniscin, a beta-defensin from the penguin stomach. Journal of Biological Chemistry 2004, 279(29):30433-30439.

43. Bailleul G, Kravtzoff A, Joulin-Giet A, Lecaille F, Labas V, Meudal H, Loth K, Teixeira-Gomes AP, Gilbert FB, Coquet L et al: The unusual resistance of avian defensin AvBD7 to proteolytic enzymes preserves its antibacterial activity. PLoS One 2016, 11(8): 0161573.

44. Harwig SS, Swiderek KM, Kokryakov VN, Tan L, Lee TD, Panyutich EA, Aleshina GM, Shamova OV, Lehrer RI: Gallinacins: cysteine-rich antimicrobial peptides of chicken leukocytes. FEBS Letters 1994, 342(3):281-285.

45. Van Dijk A, Veldhuizen EJ, Haagsman HP: Avian defensins. Veterinary Immunology and Immunopathology 2008, 124(1-2):1-18. 
46. Lynn DJ, Higgs R, Gaines S, Tierney J, James T, Lloyd AT, Fares MA, Mulcahy G, O'Farrelly C: Bioinformatic discovery and initial characterisation of nine novel antimicrobial peptide genes in the chicken. Immunogenetics 2004, 56(3):170-177.

47. Ebers KL, Zhang CY, Zhang MZ, Bailey RH, Zhang S: Transcriptional profiling avian beta-defensins in chicken oviduct epithelial cells before and after infection with Salmonella enterica serovar Enteritidis. BMC Microbiology 2009, 9:153.

48. Hong YH, Song W, Lee SH, Lillehoj HS: Differential gene expression profiles of $\beta$-defensins in the crop, intestine, and spleen using a necrotic enteritis model in 2 commercial broiler chicken lines. Poultry Science 2012, 91(5):1081-1088.

49. Zhang L, Lu L, Li S, Zhang G, Ouyang L, Robinson K, Tang Y, Zhu Q, Li D, Hu Y et al: 1,25-Dihydroxyvitamin-D3 Induces avian $\beta$-defensin gene expression in chickens. PLoS ONE 2016, 11(5):e0154546.

50. Harmon BG: Avian heterophils in inflammation and disease resistance. Poultry Science 1998, 77(7):972-977.

51. Zhang G, Sunkara LT: Avian antimicrobial host defense peptides: from biology to therapeutic applications. Pharmaceuticals (Basel) 2014, 7(3):220-247.

52. Yacoub HA, Elazzazy AM, Abuzinadah OA, Al-Hejin AM, Mahmoud MM, Harakeh SM: Antimicrobial activities of chicken beta-defensin (4 and 10) peptides against pathogenic bacteria and fungi. Frontiers in Cellular and Infection Microbiology 2015, 5:36.

53. Cheng Y, Prickett MD, Gutowska W, Kuo R, Belov K, Burt DW: Evolution of the avian beta-defensin and cathelicidin genes. BMC Evolutionary Biology 2015, $15: 188$. 
54. Lee MO, Jang H-J, Rengaraj D, Yang S-Y, Han JY, Lamont SJ, Womack JE: Tissue expression and antibacterial activity of host defense peptides in chicken. $B M C$ Veterinary Research 2016, 12:231.

55. Cuperus T, Coorens M, Van Dijk A, Haagsman HP: Avian host defense peptides. Developmental \& Comparative Immunology 2013, 41(3):352-369.

56. Yang M, Zhang $\mathrm{C}$, Zhang MZ, Zhang S: Novel synthetic analogs of avian $\beta$ defensin-12: the role of charge, hydrophobicity, and disulfide bridges in biological functions. BMC Microbiology 2017, 17(1):43.

57. Zhao L, Yang M, Zhang M, Zhang S: Expression, purification, and in vitro comparative characterization of avian beta-defensin-2, -6, and -12. Avian Disesase 2014, 58(4):541-549.

58. Derache C, Labas V, Aucagne V, Meudal H, Landon C, Delmas AF, Magallon T, Lalmanach AC: Primary structure and antibacterial activity of chicken bone marrow-derived beta-defensins. Antimicrobial Agents and Chemotherapy 2009, 53(11):4647-4655.

59. Yang M, Zhang C, Zhang X, Zhang MZ, Rottinghaus GE, Zhang S: Structurefunction analysis of Avian beta-defensin- 6 and beta-defensin-12: role of charge and disulfide bridges. BMC Microbiology 2016, 16:210.

60. Bals R, Goldman MJ, Wilson JM: Mouse $\beta$-Defensin 1 Is a Salt-sensitive antimicrobial peptide present in epithelia of the lung and urogenital tract. Infection and Immunity 1998, 66(3):1225-1232. 
61. Bals R, Wang X, Wu Z, Freeman T, Bafna V, Zasloff M, Wilson JM: Human betadefensin 2 is a salt-sensitive peptide antibiotic expressed in human lung. Journal of Clinical Investigation 1998, 102(5):874-880.

62. Ma D, Wang R, Liao W, Han Z, Liu S: Identification and characterization of a novel antibacterial peptide, avian beta-defensin 2 from ducks. Journal of Microbiology 2009, 47(5):610-618.

63. Yang D, Chertov O, Bykovskaia SN, Chen Q, Buffo MJ, Shogan J, Anderson M, Schroder JM, Wang JM, Howard OM: Beta-defensins: linking innate and adaptive immunity through dendritic and T cell CCR6. Science 1999, 286.

64. Wu Z, Hoover DM, Yang D, Boulegue C, Santamaria F, Oppenheim JJ, Lubkowski J, Lu W: Engineering disulfide bridges to dissect antimicrobial and chemotactic activities of human beta-defensin 3. Proceedings of the National Academy of Sciences of the United States of America 2003, 100(15):8880-8885.

65. Soman SS, Nair S, Issac A, Arathy DS, Niyas KP, Anoop M, Sreekumar E: Immunomodulation by duck defensin, Apl_AvBD2: in vitro dendritic cell immunoreceptor (DCIR) mRNA suppression, and B- and T-lymphocyte chemotaxis. Molecular Immunology 2009, 46.

66. Soman SS, Arathy DS, Sreekumar E: Discovery of Anas platyrhynchos avian $\beta$ defensin 2 (Apl_AvBD2) with antibacterial and chemotactic functions. Molecular Immunology 2009, 46(10):2029-2038.

67. Yurong Y, Yibao J, Ruiping S, Kaisong P, Xuemei Z, Qingqiang Y, Decheng W, Tianlong L, Huihui B: Effects of Gal-13 on the content of immunoglobulin, proliferation of lymphocyte and antibody titers after vaccination with infectious 
bursal disease virus vaccine in chickens. Asian-Australasian Journal of Animal Sciences 2007, 20(3):405-411.

68. Lee MO, Jang HJ, Rengaraj D, Yang SY, Han JY, Lamont SJ, Womack JE: Tissue expression and antibacterial activity of host defense peptides in chicken. $B M C$ Veterinary Research 2016, 12(1):231.

69. Yacoub HA, El-Hamidy SM, Mahmoud MM, Baeshen MN, Almehdar HA, Uversky VN, Redwan EM, Al-Maghrabi OA, Elazzazy AM: Biocidal activity of chicken defensin-9 against microbial pathogens. Biochemistry and Cell Biology 2016, 94(2):176-187.

70. Tu J, Qi K, Xue T, Wei H, Zhang Y, Wu Y, Zhou X, Lv X: Construction of recombinant pichia pastoris carrying a constitutive AvBD9 gene and analysis of its activity. Journal of Microbiology and Biotechnology 2015, 25(12):2082-2089.

71. Lu S, Peng K, Gao Q, Xiang M, Liu H, Song H, Yang K, Huang H, Xiao K: Molecular cloning, characterization and tissue distribution of two ostrich betadefensins: AvBD2 and AvBD7. Gene 2014, 552(1):1-7.

72. Tanwar J, Das S, Fatima Z, Hameed S: Multidrug resistance: an emerging crisis. Interdisciplinary Perspectives on Infectious Diseases 2014, 2014:541340.

73. Rai M, Pandit R, Gaikwad S, Kovics G: Antimicrobial peptides as natural biopreservative to enhance the shelf-life of food. Journal of Food Science and Technology 2016, 53(9):3381-3394.

74. Nicholls EF, Madera L, Hancock RE: Immunomodulators as adjuvants for vaccines and antimicrobial therapy. Annals of the New York Academy of Sciences 2010, 1213:46-61. 
75. Van Dijk A, Veldhuizen EJA, Kalkhove SIC, Tjeerdsma-van Bokhoven JLM, Romijn RA, Haagsman HP: The $\beta$-defensin gallinacin- 6 is expressed in the chicken digestive tract and has antimicrobial activity against food-borne pathogens. Antimicrobial Agents and Chemotherapy 2007, 51(3):912-922.

76. Chu HL, Yu HY, Yip BS, Chih YH, Liang CW, Cheng HT, Cheng JW: Boosting salt resistance of short antimicrobial peptides. Antimicrobial Agents and Chemotherapy 2013, 57(8):4050-4052.

77. Westerhoff HV, Juretic D, Hendler RW, Zasloff M: Magainins and the disruption of membrane-linked free-energy transduction. Proceedings of the National Academy of Sciences of the United States of America 1989, 86(17):6597-6601.

78. Subbalakshmi C, Nagaraj R, Sitaram N: Biological activities of C-terminal 15residue synthetic fragment of melittin: design of an analog with improved antibacterial activity. FEBS Letters 1999, 448(1):62-66.

79. Li T, Guo F, Wang Q, Fang H, Li Z, Wang D, Wang H: N-terminus three residues deletion mutant of human beta-defensin 3 with remarkably enhanced salt-resistance. PLoS One 2015, 10(2):e0117913.

80. Zhang SK, Song JW, Gong F, Li SB, Chang HY, Xie HM, Gao HW, Tan YX, Ji SP: Design of an alpha-helical antimicrobial peptide with improved cell-selective and potent anti-biofilm activity. Scientific Reports 2016, 6:27394.

81. Yin LM, Edwards MA, Li J, Yip CM, Deber CM: Roles of hydrophobicity and charge distribution of cationic antimicrobial peptides in peptide-membrane interactions. Journal of Biological Chemistry 2012, 287(10):7738-7745. 
82. Dathe M, Wieprecht T, Nikolenko H, Handel L, Maloy WL, MacDonald DL, Beyermann M, Bienert M: Hydrophobicity, hydrophobic moment and angle subtended by charged residues modulate antibacterial and haemolytic activity of amphipathic helical peptides. FEBS Letters 1997, 403(2):208-212.

83. Mohamed MF, Abdelkhalek A, Seleem MN: Evaluation of short synthetic antimicrobial peptides for treatment of drug-resistant and intracellular Staphylococcus aureus. Scientific Reports 2016, 6:29707.

84. Ulm H, Wilmes M, Shai Y, Sahl HG: Antimicrobial host defensins - specific antibiotic activities and innate defense modulation. Frontiers in Immunology 2012, $3: 249$.

85. Na D, Qing-Quan M, An-Shan S, Yin-Feng L, Wanning H, Yao G, Yu-Zhi L: Novel design of short antimicrobial peptides derived from the bactericidal domain of avian beta-defensin-4. Protein \& Peptide Letters 2012, 19(11):1212-1219.

86. Klüver E, Schulz-Maronde S, Scheid S, Meyer B, Forssmann W-G, Adermann K: Structure-activity relation of human $\beta$-defensin 3: influence of disulfide bonds and cysteine substitution on antimicrobial activity and cytotoxicity. Biochemistry 2005, 44(28):9804-9816.

87. Chan DI, Prenner EJ, Vogel HJ: Tryptophan- and arginine-rich antimicrobial peptides: structures and mechanisms of action. Biochimica et Biophysica Acta 2006, 1758(9):1184-1202.

88. Liu Z, Brady A, Young A, Rasimick B, Chen K, Zhou C, Kallenbach NR: Length effects in antimicrobial peptides of the (RW)n series. Antimicrobial Agents and Chemotherapy 2007, 51(2):597-603. 
89. Yu HY, Tu CH, Yip BS, Chen HL, Cheng HT, Huang KC, Lo HJ, Cheng JW: Easy strategy to increase salt resistance of antimicrobial peptides. Antimicrobial Agents and Chemotherapy 2011, 55(10):4918-4921.

90. Stromstedt AA, Pasupuleti M, Schmidtchen A, Malmsten M: Evaluation of strategies for improving proteolytic resistance of antimicrobial peptides by using variants of EFK17, an internal segment of LL-37. Antimicrobial Agents and Chemotherapy 2009, 53(2):593-602.

91. Nell MJ, Tjabringa GS, Wafelman AR, Verrijk R, Hiemstra PS, Drijfhout JW, Grote JJ: Development of novel LL-37 derived antimicrobial peptides with LPS and LTA neutralizing and antimicrobial activities for therapeutic application. Peptides 2006, 27(4):649-660.

92. Matsuzaki K: Control of cell selectivity of antimicrobial peptides. Biochimica et Biophysica Acta (BBA) - Biomembranes 2009, 1788(8):1687-1692.

93. Olli S, Nagaraj R, Motukupally SR: A hybrid cationic peptide composed of human beta-defensin-1 and humanized theta-defensin sequences exhibits salt-resistant antimicrobial activity. Antimicrobial Agents and Chemotherapy 2015, 59(1):217225.

94. Park IY, Cho JH, Kim KS, Kim YB, Kim MS, Kim SC: Helix stability confers salt resistance upon helical antimicrobial peptides. Journal of Biological Chemistry 2004, 279(14):13896-13901.

95. Jenssen H, Fjell CD, Cherkasov A, Hancock RE: QSAR modeling and computeraided design of antimicrobial peptides. Journal of Peptide Science 2008, 14(1):110114. 
96. Bulet P, Stocklin R, Menin L: Anti-microbial peptides: from invertebrates to vertebrates. Immunological Reviews 2004, 198:169-184.

97. Yang D, Biragyn A, Hoover DM, Lubkowski J, Oppenheim JJ: Multiple roles of antimicrobial defensins, cathelicidins, and eosinophil-derived neurotoxin in host defense. Annual Review of Immunology 2004, 22:181-215.

98. Verma C, Seebah S, Low SM, Zhou L, Liu SP, Li J, Beuerman RW: Defensins: antimicrobial peptides for therapeutic development. Biotechnology Journal 2007, 2(11):1353-1359.

99. Hancock RE, Nijnik A, Philpott DJ: Modulating immunity as a therapy for bacterial infections. Nature Reviews Microbiology 2012, 10(4):243-254.

100. Hancock RE: Peptide antibiotics. Lancet 1997, 349(9049):418-422.

101. Palffy R, Gardlik R, Behuliak M, Kadasi L, Turna J, Celec P: On the physiology and pathophysiology of antimicrobial peptides. Molecular Medicine 2009, 15(12):51-59.

102. Pokorny A, Almeida PF: Permeabilization of raft-containing lipid vesicles by deltalysin: a mechanism for cell sensitivity to cytotoxic peptides. Biochemistry 2005, 44(27):9538-9544.

103. Semple F, MacPherson H, Webb S, Cox SL, Mallin LJ, Tyrrell C, Grimes GR, Semple CA, Nix MA, Millhauser GL et al: Human beta-defensin 3 affects the activity of pro-inflammatory pathways associated with MyD88 and TRIF. European Journal of Immunology 2011, 41(11):3291-3300.

104. Yang D, Chertov O, Bykovskaia SN, Chen Q, Buffo MJ, Shogan J, Anderson M, Schroder JM, Wang JM, Howard OM et al: Beta-defensins: linking innate and 
adaptive immunity through dendritic and T cell CCR6. Science 1999, 286(5439):525-528.

105. Biragyn A, Surenhu M, Yang D, Ruffini PA, Haines BA, Klyushnenkova E, Oppenheim JJ, Kwak LW: Mediators of innate immunity that target immature, but not mature, dendritic cells induce antitumor immunity when genetically fused with nonimmunogenic tumor antigens. Journal of Immunology 2001, 167(11):66446653.

106. Rohrl J, Yang D, Oppenheim JJ, Hehlgans T: Human beta-defensin 2 and 3 and their mouse orthologs induce chemotaxis through interaction with CCR2. Journal of Immunology 2010, 184(12):6688-6694.

107. Biragyn A, Coscia M, Nagashima K, Sanford M, Young HA, Olkhanud P: Murine beta-defensin 2 promotes TLR-4/MyD88-mediated and NF-kappaB-dependent atypical death of APCs via activation of TNFR2. Journal of Leukocyte Biology 2008, 83(4):998-1008.

108. Yang Y, Jiang Y, Yin Q, Liang H, She R: Chicken intestine defensins activated murine peripheral blood mononuclear cells through the TLR4-NF-kappaB pathway. Veterinary Immunology and Immunopathology 2010, 133(1):59-65.

109. Zhang HH, Yang XM, Xie QM, Ma JY, Luo YN, Cao YC, Chen F, Bi YZ: The potent adjuvant effects of chicken beta-defensin-1 when genetically fused with infectious bursal disease virus VP2 gene. Veterinary Immunology and Immunopathology 2010, 136(1-2):92-97.

110. Ferris LK, Mburu YK, Mathers AR, Fluharty ER, Larregina AT, Ferris RL, Falo LD, Jr.: Human beta-defensin 3 induces maturation of human langerhans cell-like 
dendritic cells: an antimicrobial peptide that functions as an endogenous adjuvant. Journal of Investigative Dermatology 2013, 133(2):460-468.

111. Lynn DJ, Higgs R, Lloyd AT, O'Farrelly C, Herve-Grepinet V, Nys Y, Brinkman FS, Yu PL, Soulier A, Kaiser P et al: Avian beta-defensin nomenclature: a community proposed update. Immunology Letters 2007, 110(1):86-89.

112. Lehrer RI, Ganz T: Defensins of vertebrate animals. Current Opinion in Immunology 2002, 14(1):96-102.

113. Klotman ME, Chang TL: Defensins in innate antiviral immunity. Nature Revierws Immunology 2006, 6(6):447-456.

114. Evans EW, Beach GG, Wunderlich J, Harmon BG: Isolation of antimicrobial peptides from avian heterophils. Journal of Leukocyte Biology 1994, 56(5):661-665.

115. Zhao C, Nguyen T, Liu L, Sacco RE, Brogden KA, Lehrer RI: Gallinacin-3, an inducible epithelial beta-defensin in the chicken. Infection and Immunology 2001, 69(4):2684-2691.

116. Kluver E, Adermann K, Schulz A: Synthesis and structure-activity relationship of beta-defensins, multi-functional peptides of the immune system. Journal of Peptide Science 2006, 12(4):243-257.

117. Institute CLSI: Methods for dilution antimicrobial susceptibility tests for bacteria that grow aerobically, approved standard, 8th ed. CLSI publication M07-A8. 2009.

118. Institute CLSI: Performance standards for antimicrobial susceptibility testing, 22nd informational supplement. CLSI publication M100-S22. 2012. 
119. Falk W, Goodwin RH, Jr., Leonard EJ: A 48-well micro chemotaxis assembly for rapid and accurate measurement of leukocyte migration. Journal of Immunological Methods 1980, 33(3):239-247.

120. Park IK, Kang DH: Effect of electropermeabilization by ohmic heating for inactivation of Escherichia coli O157:H7, Salmonella enterica serovar Typhimurium, and Listeria monocytogenes in buffered peptone water and apple juice. Applied and Environmental Microbiology 2013, 79(23):7122-7129.

121. Hoover DM, Wu Z, Tucker K, Lu W, Lubkowski J: Antimicrobial characterization of human beta-defensin 3 derivatives. Antimicrobial Agents and Chemotherapy 2003, 47(9):2804-2809.

122. Krishnakumari V, Sharadadevi A, Singh S, Nagaraj R: Single disulfide and linear analogs corresponding to the carboxy-terminal segment of bovine beta-defensin-2: effects of introducing the beta-hairpin nucleating sequence d-pro-gly on antibacterial activity and Biophysical properties. Biochemistry 2003, 42(31):93079315.

123. Kluver E, Schulz-Maronde S, Scheid S, Meyer B, Forssmann WG, Adermann K: Structure-activity relation of human beta-defensin 3: influence of disulfide bonds and cysteine substitution on antimicrobial activity and cytotoxicity. Biochemistry 2005, 44(28):9804-9816.

124. Mandal M, Jagannadham MV, Nagaraj R: Antibacterial activities and conformations of bovine beta-defensin BNBD-12 and analogs:structural and disulfide bridge requirements for activity. Peptides 2002, 23(3):413-418. 
125. Holmgren A: Enzymatic reduction-oxidation of protein disulfides by thioredoxin. Methods in Enzymology 1984, 107:295-300.

126. Scudiero O, Galdiero S, Cantisani M, Di Noto R, Vitiello M, Galdiero M, Naclerio G, Cassiman JJ, Pedone C, Castaldo G et al: Novel synthetic, salt-resistant analogs of human beta-defensins 1 and 3 endowed with enhanced antimicrobial activity. Antimicrobial Agents and Chemotherapy 2010, 54(6):2312-2322.

127. Schroeder BO, Wu Z, Nuding S, Groscurth S, Marcinowski M, Beisner J, Buchner J, Schaller M, Stange EF, Wehkamp J: Reduction of disulphide bonds unmasks potent antimicrobial activity of human beta-defensin 1. Nature 2011, 469(7330):419-423.

128. Cao Y, Ma Q, Shan A, Dong N: Expression in Pichia Pastoris and biological activity of avian $\beta$-defensin 6 and its mutant peptide without cysteines. Protein and Peptide Letters 2012, 19(10):1064-1070.

129. Lepper PM, Held TK, Schneider EM, Bolke E, Gerlach H, Trautmann M: Clinical implications of antibiotic-induced endotoxin release in septic shock. Intensive Care Medicine 2002, 28(7):824-833.

130. Motzkus D, Schulz-Maronde S, Heitland A, Schulz A, Forssmann WG, Jubner M, Maronde E: The novel beta-defensin DEFB123 prevents lipopolysaccharidemediated effects in vitro and in vivo. The FASEB Journal 2006, 20(10):1701-1702.

131. Yu H, Dong J, Gu Y, Liu H, Xin A, Shi H, Sun F, Zhang Y, Lin D, Diao H: The novel human beta-defensin 114 regulates lipopolysaccharide (LPS)-mediated inflammation and protects sperm from motility loss. Journal of Biological Chemistry 2013, 288(17):12270-12282. 
132. Takahashi D, Shukla SK, Prakash O, Zhang G: Structural determinants of host defense peptides for antimicrobial activity and target cell selectivity. Biochimie 2010, 92(9):1236-1241.

133. Lai Y, Gallo RL: AMPed up immunity: how antimicrobial peptides have multiple roles in immune defense. Trends in Immunology 2009, 30(3):131-141.

134. Kaiser P, Poh TY, Rothwell L, Avery S, Balu S, Pathania US, Hughes S, Goodchild M, Morrell S, Watson M et al: A genomic analysis of chicken cytokines and chemokines. Journal of Interferon \& Cytokine Research 2005, 25(8):467-484.

135. Munoz I, Berges M, Bonsergent C, Cormier-Aline F, Quere P, Sibille P: Cloning, expression and functional characterization of chicken CCR6 and its ligand CCL20. Molecular Immunology 2009, 47(2-3):551-559.

136. Ai LS, Lee SF, Chen SS, Liao F: Molecular characterization of CCR6: involvement of multiple domains in ligand binding and receptor signaling. Journal of Biomedical science 2004, 11(6):818-828.

137. Meincken M, Holroyd DL, Rautenbach M: Atomic force microscopy study of the effect of antimicrobial peptides on the cell envelope of Escherichia coli. Antimicrobial Agents and Chemotherapy 2005, 49(10):4085-4092.

138. Van Dijk A, Veldhuizen EJ, Kalkhove SI, Tjeerdsma-van Bokhoven JL, Romijn RA, Haagsman HP: The beta-defensin gallinacin-6 is expressed in the chicken digestive tract and has antimicrobial activity against food-borne pathogens. Antimicrobial Agents and Chemotherapy 2007, 51(3):912-922.

139. Hartmann M, Berditsch M, Hawecker J, Ardakani MF, Gerthsen D, Ulrich AS: Damage of the bacterial cell envelope by antimicrobial peptides gramicidin $\mathrm{S}$ and 
PGLa as revealed by transmission and scanning electron microscopy. Antimicrobial Agents and Chemotherapy 2010, 54(8):3132-3142.

140. Joshi S, Bisht GS, Rawat DS, Kumar A, Kumar R, Maiti S, Pasha S: Interaction studies of novel cell selective antimicrobial peptides with model membranes and $E$. coli ATCC 11775. Biochimica et Biophysica Acta 2010, 1798(10):1864-1875.

141. Wang W, Tao R, Tong Z, Ding Y, Kuang R, Zhai S, Liu J, Ni L: Effect of a novel antimicrobial peptide chrysophsin-1 on oral pathogens and Streptococcus mutans biofilms. Peptides 2012, 33(2):212-219.

142. Peng KS, Ruan LS, Tu J, Qi KZ, Jiang LH: Tissue distribution, expression, and antimicrobial activity of Anas platyrhynchos avian beta-defensin 6. Poultry Science 2013, 92(1):97-104.

143. Donoghue DJ: Antibiotic residues in poultry tissues and eggs: human health concerns? Poultry Science 2003, 82(4):618-621.

144. Catry B, Laevens H, Devriese LA, Opsomer G, De Kruif A: Antimicrobial resistance in livestock. Journal of Veterinary Pharmacology and Therapeutics 2003, 26(2):81-93.

145. Soulsby EJ: Resistance to antimicrobials in humans and animals. British Medical Journal (Clinical Research Education) 2005, 331(7527):1219-1220.

146. Ventola CL: The antibiotic resistance crisis: part 2: management strategies and new agents. Pharmacy and Therapeutics 2015, 40(5):344-352.

147. Aoki W, Ueda M: Characterization of antimicrobial peptides toward the development of novel antibiotics. Pharmaceuticals (Basel) 2013, 6(8):1055-1081. 
148. Ma D, Lin L, Zhang K, Han Z, Shao Y, Liu X, Liu S: Three novel Anas platyrhynchos avian beta-defensins, upregulated by duck hepatitis virus, with antibacterial and antiviral activities. Molecular Immunology 2011, 49(1-2):84-96.

149. French GL: Bactericidal agents in the treatment of MRSA infections--the potential role of daptomycin. Journal of Antimicrobial Chemotherapy 2006, 58(6):11071117.

150. Cobo ER, Kissoon-Singh V, Moreau F, Chadee K: Colonic MUC2 mucin regulates the expression and antimicrobial activity of beta-defensin 2. Mucosal immunology 2015, 8(6):1360-1372.

151. De Paula VS, Gomes NS, Lima LG, Miyamoto CA, Monteiro RQ, Almeida FC, Valente AP: Structural basis for the interaction of human beta-defensin 6 and its putative chemokine receptor CCR2 and breast cancer microvesicles. Journal of Molecular Biology 2013, 425(22):4479-4495.

152. Dragelj JL, Stanković IM, Božinovski DM, Meyer T, Veljković DŽ, Medaković VB, Knapp E-W, Zarić SD: C-H/O interactions of aromatic ch donors within proteins: a crystallographic study. Crystal Growth \& Design 2016, 16(4):19481957.

153. Soman SS, Nair S, Issac A, Arathy DS, Niyas KP, Anoop M, Sreekumar E: Immunomodulation by duck defensin, Apl_AvBD2: in vitro dendritic cell immunoreceptor (DCIR) mRNA suppression, and B- and T-lymphocyte chemotaxis. Molecular Immunology 2009, 46(15):3070-3075. 
154. Shinnar AE, Butler KL, Park HJ: Cathelicidin family of antimicrobial peptides: proteolytic processing and protease resistance. Bioorganic Chemistry 2003, 31(6):425-436.

155. Jin T, Bokarewa M, Foster T, Mitchell J, Higgins J, Tarkowski A: Staphylococcus aureus resists human defensins by production of staphylokinase, a novel bacterial evasion mechanism. Journal of Immunology 2004, 172(2):1169-1176.

156. Ernst CM, Staubitz P, Mishra NN, Yang SJ, Hornig G, Kalbacher H, Bayer AS, Kraus D, Peschel A: The bacterial defensin resistance protein MprF consists of separable domains for lipid lysinylation and antimicrobial peptide repulsion. PLoS Pathogens 2009, 5(11):e1000660.

157. Nawrocki KL, Crispell EK, McBride SM: Antimicrobial peptide resistance mechanisms of gram-positive bacteria. Antibiotics (Basel, Switzerland) 2014, 3(4):461-492.

158. Malanovic N, Lohner K: Gram-positive bacterial cell envelopes: The impact on the activity of antimicrobial peptides. Biochimica et Biophysica Acta 2016, 1858(5):936-946.

159. Cole JN, Nizet V: Bacterial evasion of host antimicrobial peptide defenses. Microbiology Spectrum 2016, 4(1).

160. Joo HS, Fu CI, Otto M: Bacterial strategies of resistance to antimicrobial peptides. Philosophical transactions of the Royal Society of London Series B, Biological sciences 2016, 371(1695): 20150292.

161. Mohanram H, Bhattacharjya S: Salt-resistant short antimicrobial peptides. Biopolymers 2016, 106(3):345-356. 
162. Straus SK, Hancock REW: Mode of action of the new antibiotic for Gram-positive pathogens daptomycin: Comparison with cationic antimicrobial peptides and lipopeptides. Biochimica et Biophysica Acta (BBA) - Biomembranes 2006, 1758(9):1215-1223.

163. Jenssen H, Hamill P, Hancock RE: Peptide antimicrobial agents. Clinical Microbiology Reviews 2006, 19(3):491-511.

164. Greber KE, Dawgul M: Antimicrobial peptides under clinical trials. Current Topics in Medicinal Chemistry 2016.

165. Rozek A, Powers JP, Friedrich CL, Hancock RE: Structure-based design of an indolicidin peptide analog with increased protease stability. Biochemistry 2003, 42(48):14130-14138.

166. Giangaspero A, Sandri L, Tossi A: Amphipathic alpha helical antimicrobial peptides. European Journal of Biochemistry 2001, 268(21):5589-5600.

167. Mohanram H, Bhattacharjya S: Resurrecting inactive antimicrobial peptides from the lipopolysaccharide trap. Antimicrobial Agents and Chemotherapy 2014, 58(4):1987-1996.

168. Jerala R: Synthetic lipopeptides: a novel class of anti-infectives. Expert Opinion on Investigational Drugs 2007, 16(8):1159-1169.

169. Chu-Kung AF, Nguyen R, Bozzelli KN, Tirrell M: Chain length dependence of antimicrobial peptide-fatty acid conjugate activity. Journal of Colloid and Interface Science 2010, 345(2):160-167.

170. Vagner J, Qu H, Hruby VJ: Peptidomimetics, a synthetic tool of drug discovery. Current Opinion in Chemical Biology 2008, 12(3):292-296. 
171. Ringstad L, Kacprzyk L, Schmidtchen A, Malmsten M: Effects of topology, length, and charge on the activity of a kininogen-derived peptide on lipid membranes and bacteria. Biochimica et Biophysica Acta 2007, 1768(3):715-727.

172. Saravanan R, Li X, Lim K, Mohanram H, Peng L, Mishra B, Basu A, Lee JM, Bhattacharjya S, Leong SS: Design of short membrane selective antimicrobial peptides containing tryptophan and arginine residues for improved activity, saltresistance, and biocompatibility. Biotechnology and Bioengineering 2014, 111(1):37-49

173. Kim H, Jang JH, Kim SC, Cho JH: De novo generation of short antimicrobial peptides with enhanced stability and cell specificity. Journal of Antimicrobial Chemotherapy 2014, 69(1):121-132.

174. Tyagi P, Singh M, Kumari H, Kumari A, Mukhopadhyay K: Bactericidal activity of curcumin I is associated with damaging of bacterial membrane. PLoS ONE 2015, 10(3):e0121313.

175. Zhang S-K, Song J-w, Gong F, Li S-B, Chang H-Y, Xie H-M, Gao H-W, Tan YX, Ji S-P: Design of an $\alpha$-helical antimicrobial peptide with improved cell-selective and potent anti-biofilm activity. Scientific Report 2016, 6:27394.

176. Kuhlmann KFD, Van Till JWO, Boermeester MA, de Reuver PR, Tzvetanova ID, Offerhaus GJA, ten Kate FJW, Busch ORC, Van Gulik TM, Gouma DJ et al: Evaluation of matrix metalloproteinase 7 in plasma and pancreatic juice as a biomarker for pancreatic cancer. Cancer epidemiology, biomarkers \& prevention : a publication of the American Association for Cancer Research, cosponsored by the American Society of Preventive Oncology 2007, 16(5):886-891. 
177. Forde E, Humphreys H, Greene CM, Fitzgerald-Hughes D, Devocelle M: Potential of host defense peptide prodrugs as neutrophil elastase-dependent anti-infective agents for cystic fibrosis. Antimicrobial Agents and Chemotherapy 2014, 58(2):978-985.

178. Doxakis A, Maria A, Savvas P, Zafiroula I: Assessment of the roles of cathepsins B, H and L in the progression of colorectal cancer. Journal of Cancer Therapy 2013, $4: 1-7$.

179. Nguyen LT, Chau JK, Perry NA, de Boer L, Zaat SA, Vogel HJ: Serum stabilities of short tryptophan- and arginine-rich antimicrobial peptide analogs. PLoS One 2010, 5(9).

180. Hansen A, Schafer I, Knappe D, Seibel P, Hoffmann R: Intracellular toxicity of proline-rich antimicrobial peptides shuttled into mammalian cells by the cellpenetrating peptide penetratin. Antimicrobial Agents and Chemotherapy 2012, 56(10):5194-5201.

181. Gopal R, Park JS, Seo CH, Park Y: Applications of circular dichroism for structural analysis of gelatin and antimicrobial peptides. International Journal of Molecular Sciences 2012, 13(3):3229-3244.

182. Shinnar AE, Butler KL, Park HJ: Cathelicidin family of antimicrobial peptides: proteolytic processing and protease resistance. Bioorganic Chemistry 2003, 31.

183. Lefkowitz RB, Schmid-Schonbein GW, Heller MJ: Whole blood assay for elastase, chymotrypsin, matrix metalloproteinase-2, and matrix metalloproteinase-9 activity. Analytical Chemistry 2010, 82(19):8251-8258. 
184. Iwaki K, Ogawa M, Tanaka S, Kosaki G: Radioimmunoassay for human pancreatic chymotrypsin and measurement of serum immunoreactive chymotrypsin contents in various diseases. Research Communications in Chemical Pathology and Pharmacology 1983, 40(3):489-496.

185. Matsuzaki K, Sugishita K-i, Harada M, Fujii N, Miyajima K: Interactions of an antimicrobial peptide, magainin 2, with outer and inner membranes of Gramnegative bacteria. Biochimica et Biophysica Acta (BBA) - Biomembranes 1997, 1327(1):119-130.

186. Mora-Navarro C, Méndez-Vega J, Caraballo-León J, Lee M-r, Palecek S, TorresLugo M, Ortiz-Bermúdez P: Hydrophobicity of antifungal $\beta$-peptides is associated with their cytotoxic effect on in vitro human colon Caco-2 and liver HepG2 cells. PLoS ONE 2016, 11(3):e0149271.

187. Kluver E, Schulz-Maronde S, Scheid S, Meyer B, Forssmann WG, Adermann K: Structure-activity relation of human beta-defensin 3: influence of disulfide bonds and cysteine substitution on antimicrobial activity and cytotoxicity. Biochemistry $2005,44$.

188. Tjabringa GS, Ninaber DK, Drijfhout JW, Rabe KF, Hiemstra PS: Human Cathelicidin LL-37 Is a Chemoattractant for Eosinophils and Neutrophils That Acts via formyl-peptide receptors. International Archives of Allergy and Immunology 2006, 140(2):103-112.

189. Pundir P, Catalli A, Leggiadro C, Douglas SE, Kulka M: Pleurocidin, a novel antimicrobial peptide, induces human mast cell activation through the FPRL1 receptor. Mucosal immunology 2014, 7(1):177-187. 
190. Balasubramanian D, Kumari H, Mathee K: Pseudomonas aeruginosa AmpR: an acute-chronic switch regulator. Pathogens and Disease 2015, 73(2):1-14.

191. Hogardt M, Heesemann J: Microevolution of Pseudomonas aeruginosa to a chronic pathogen of the cystic fibrosis lung. Current Topics in Microbiology and Immunology 2013, 358:91-118.

192. Poole K: Pseudomonas Aeruginosa: Resistance to the Max. Frontiers in Microbiology 2011, 2:65.

193. Church D, Elsayed S, Reid O, Winston B, Lindsay R: Burn wound infections. Clinical Microbiology Reviews 2006, 19(2):403-434.

194. Lister PD, Wolter DJ, Hanson ND: Antibacterial-resistant Pseudomonas aeruginosa: clinical impact and complex regulation of chromosomally encoded resistance mechanisms. Clinical Microbiology Reviews 2009, 22(4):582-610.

195. Hirsch EB, Tam VH: Impact of multidrug-resistant Pseudomonas aeruginosa infection on patient outcomes. Expert Review of Pharmacoeconomics \& Outcomes Research 2010, 10(4):441-451.

196. Hogberg LD, Heddini A, Cars O: The global need for effective antibiotics: challenges and recent advances. Trends in Pharmacological Sciences 2010, 31(11):509-515.

197. Uhlig T, Kyprianou T, Martinelli FG, Oppici CA, Heiligers D, Hills D, Calvo XR, Verhaert P: The emergence of peptides in the pharmaceutical business: From exploration to exploitation. EuPA Open Proteomics 2014, 4:58-69.

198. Fox JL: Antimicrobial peptides stage a comeback. Nature Biotechnology 2013, 31(5):379-382. 
199. National Research Council Committee for the Update of the Guide for the C, Use of laboratory A: the national academies collection: reports funded by national institutes of health. In: Guide for the Care and Use of Laboratory Animals. Edited by th. Washington (DC): National Academies Press (US) National Academy of Sciences, 2011.

200. Watters C, DeLeon K, Trivedi U, Griswold JA, Lyte M, Hampel KJ, Wargo MJ, Rumbaugh KP: Pseudomonas aeruginosa biofilms perturb wound resolution and antibiotic tolerance in diabetic mice. Medical Microbiology and Immunology 2013, 202(2):131-141.

201. Hakansson J, Bjorn C, Lindgren K, Sjostrom E, Sjostrand V, Mahlapuu M: Efficacy of the novel topical antimicrobial agent PXL150 in a mouse model of surgical site infections. Antimicrobial Agents and Chemotherapy 2014, 58(5):2982-2984.

202. Van Veen SQ, Claas EC, Kuijper EJ: High-throughput identification of bacteria and yeast by matrix-assisted laser desorption ionization-time of flight mass spectrometry in conventional medical microbiology laboratories. Journal of Clinical Microbiology 2010, 48(3):900-907.

203. Proksch E, Brandner JM, Jensen JM: The skin: an indispensable barrier. Experimental Dermatology 2008, 17(12):1063-1072.

204. Schauber J, Gallo RL: Antimicrobial peptides and the skin immune defense system. The Journal of Allergy and Clinical Immunology 2008, 122(2):261-266.

205. Starr CG, He J, Wimley WC: Host cell interactions are a significant barrier to the clinical utility of peptide antibiotics. ACS Chemical Biology 2016, 11(12):33913399. 
206. Weingand K, Bloom J, Carakostas M, Hall R, Helfrich M, Latimer K, Levine B, Neptun D, Rebar A, Stitzel K et al: Clinical pathology testing recommendations for nonclinical toxicity and safety studies. AACC-DACC/ASVCP Joint Task Force. Toxicological Pathology 1992, $20(3$ Pt 2):539-543.

207. Giannini EG, Testa R, Savarino V: Liver enzyme alteration: a guide for clinicians. Canadian Medical Association Journal 2005, 172(3):367-379.

208. Lippi G, Salvagno GL, Montagnana M, Brocco G, Guidi GC. Influence of hemolysis on routine clinical chemistry testing. Clinical Chemistry and Laboratory Medicine 2006, 44 (3): 311-316.

209. Zavascki AP, Goldani LZ, Li J, Nation RL: Polymyxin B for the treatment of multidrug-resistant pathogens: a critical review. Journal of Antimicrobial Chemotherapy 2007, 60(6):1206-1215.

210. Banerjee S, Argaez C: CADTH rapid response reports. in: topical antibiotics for infection prevention: a review of the clinical effectiveness and guidelines. Ottawa (ON): Canadian Agency for Drugs and Technologies in Health Copyright (c) 2017 Canadian Agency for Drugs and Technologies in Health, 2017.

211. Falagas ME, Kasiakou SK: Toxicity of polymyxins: a systematic review of the evidence from old and recent studies. Critical Care 2006, 10(1):R27.

212. Yamaguchi Y, Yoshikawa K: Cutaneous wound healing: an update. The Journal of Dermatology 2001, 28(10):521-534.

213. Trostrup H, Thomsen K, Christophersen LJ, Hougen HP, Bjarnsholt T, Jensen PO, Kirkby N, Calum H, Hoiby N, Moser C: Pseudomonas aeruginosa biofilm 
aggravates skin inflammatory response in BALB/c mice in a novel chronic wound model. Wound Repair and Regeneration 2013, 21(2):292-299.

214. Kugelberg E, Norström T, Petersen TK, Duvold T, Andersson DI, Hughes D: Establishment of a superficial skin infection model in mice by using Staphylococcus aureus and Streptococcus pyogenes. Antimicrobial Agents and Chemotherapy 2005, 49(8):3435-3441. 


\section{VITA}

Ming Yang is from China. In 2005, Yang finished his bachelor degree in Veterinary Medical School at Agriculture University of Hebei, then he got his master degree in Clinical Veterinary Medicine at Beijing University of Agriculture (BUA). Yang continued his educational training as a visiting scholar in Mississippi State Veterinary Diagnostic Laboratory for two years from 2009 to 2011. While worked in MSU, he got married to Chunye Zhang, a lovely supporting wife for his life. All these working experiences made his decision to pursue Ph.D. in pathobiology and microbiology to contribute to understand the infecting mechanisms of bacteria and find scientific strategies for treating bacterial infections.

In 2013, he joined the Veterinary Pathobiology doctoral program in Dr. Zhang's Lab at Texas A\&M University. It was there he started to design novel antimicrobial peptides on the basis of avian beta-defensins (AvBDs), promising therapeutic agents against multipledrug-resistant bacteria. In 2014, he transferred to University of Missouri-Columbia to

continue his study. The studies on wild-type peptides and their analogs made him aware of that much efforts were needed to conquer the drawbacks of natural host AMPs, such as sensitivity to salts and proteases, and complex structure of nature AvBDs. After reading hundreds of papers, he designed criteria to develop novel short antimicrobial peptides to solve the barriers that inhibit the development of AMPs as therapeutic agents. The results of this dissertation indicated his contribution to the next generation of antibiotics. After finishing his Ph.D., he will continue his research study in the fields of microbial infection and treatment. 